\title{
An Umpolung Oxa-[2,3] Sigmatropic Rearrangement Employing Arynes for the Synthesis of Functionalized Enol Ethers
}

\author{
Rahul N. Gaykar, Malini George, Avishek Guin, Subrata Bhattacharjee, and \\ Akkattu T. Biju* \\ Department of Organic Chemistry, Indian Institute of Science, Bangalore-560012, India \\ atbiju@iisc.ac.in
}

\section{Supporting Information}

1. General Information $\quad$ S02

2. General Procedure for Synthesis of $\beta$-Keto Thioethers $\quad$ S03

3. General Procedure for the Synthesis of Functionalized Enol Ethers S05

4. Mechanistic Experiments $\quad$ S06

5. X-ray Data of $\mathbf{1 1} \quad \mathrm{S} 16$

6. Synthesis and Characterization of $\beta$-Keto Thioethers $\quad$ S18

7. Synthesis and Characterization of Functionalized Enol Ethers $\quad$ S36

8. Product Functionalization $\quad$ S64

9. ${ }^{1} \mathrm{H}$ and ${ }^{13} \mathrm{C}$ NMR Spectra of $\beta$-Keto Thioethers $\quad$ S68

10. ${ }^{1} \mathrm{H}$ and ${ }^{13} \mathrm{C}$ NMR Spectra of Functionalized Enol Ethers S106 


\section{General Information}

Unless otherwise specified, all reactions were carried out under an atmosphere of nitrogen in flame-dried reaction vessels with Teflon screw caps. $25^{\circ} \mathrm{C}$ Corresponds to the room temperature (rt) of the lab when the experiments were carried out. THF was freshly purified by distillation over Na-benzophenone and was transferred under nitrogen. 18-Crown-6 was recrystallized from dry $\mathrm{CH}_{3} \mathrm{CN}$, and $\mathrm{KF}$ was dried by heating at $110^{\circ} \mathrm{C}$ for $12 \mathrm{~h}$ and left to cool under nitrogen and stored in nitrogen filled glove-box. All the acetophenone derivatives were purchased from commercial sources and were used without further purification. The 2(trimethylsilyl)phenyl trifluoromethane sulfonate 2a and the other symmetrical and unsymmetrical aryne precursors were synthesized following the literature procedure. ${ }^{1}$

Analytical thin layer chromatography was performed on TLC Silica gel $60 \mathrm{~F}_{254}$. Visualization was accomplished with short wave UV light or $\mathrm{KMnO}_{4}$ staining solutions followed by heating. Flash chromatography was performed on silica gel (230-400 mesh) by standard techniques eluting with Pet. Ether-EtOAc solvent system.

All compounds were fully characterized. ${ }^{1} \mathrm{H}$ and ${ }^{13} \mathrm{C}$ NMR spectra were recorded on Bruker Ultrashield spectrometer in $\mathrm{CDCl}_{3}$ as solvent. Chemical shifts $(\delta)$ are given in ppm. The residual solvent signals were used as references and the chemical shifts converted to the TMS scale $\left(\mathrm{CDCl}_{3}\right.$ : $\delta_{\mathrm{H}}=7.26 \mathrm{ppm}, \delta_{\mathrm{C}}=77.16 \mathrm{ppm}$ ). Infrared (FT-IR) spectra were recorded on a Bruker alpha FT-IR spectrophotometer, $v$-max in $\mathrm{cm}^{-1}$. HRMS (ESI) data were recorded on a Waters Xevo G2-XS QTOF instrument.

(a) Sato, Y.; Tamura, T.; Kinbara, A; Morib, M. Adv. Synth. Catal. 2007, 349, 647. (b) Peña, D.; Cobas, A.; Pérez, D.; Guitián, E. Synthesis, 2002, 1454. 


\section{General Procedure for Synthesis of $\beta$-Keto Thioethers}

The $\beta$-keto thioethers used for the present study has been prepared from commercially available acetophenone derivatives following the known three-step procedure.
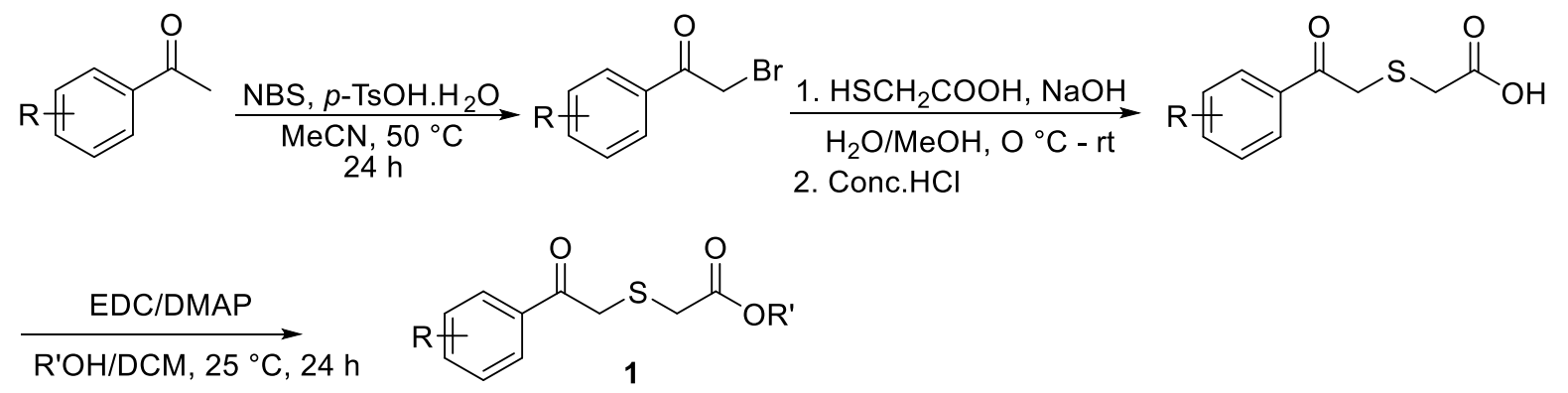

\section{Procedure for Synthesis of $\alpha$-Bromoacetophenones ${ }^{2}$}

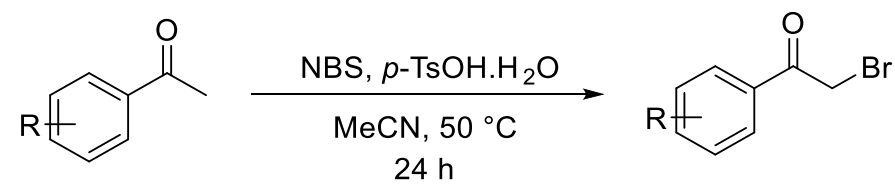

$\alpha$-Bromoacetophenones was synthesized following the literature procedure. ${ }^{2}$ To a solution of the acetophenone derivative ( $9.7 \mathrm{mmol}, 1$ equiv) in $25 \mathrm{~mL}$ of acetonitrile were added NBS (1.7 $\mathrm{g}$, $9.7 \mathrm{mmol}, 1.0$ equiv) and $p$-toluene sulfonic acid monohydrate ( $1.8 \mathrm{~g}, 9.7 \mathrm{mmol}, 1.0$ equiv). The reaction mixture was stirred for $24 \mathrm{~h}$ at $50{ }^{\circ} \mathrm{C}$ in a pre-heated oil bath. After that time, the solvent was evaporated under reduced pressure. A water solution of saturated $\mathrm{NaHCO}_{3}(30 \mathrm{~mL})$ was then added, and the solution was extracted with dichloromethane $(3 \times 30 \mathrm{~mL})$. The organic layers were combined and dried over $\mathrm{Na}_{2} \mathrm{SO}_{4}$. The solvent was evaporated, and the residue was subjected to column chromatography (silica gel) using hexanes $/ \mathrm{CH}_{2} \mathrm{Cl}_{2}$ (from 9:1 to 4:1) as eluent. These compounds exhibited physical and spectral data in agreement with those reported.

General Procedure for the Synthesis of Phenacyl Acetic Acid Derivatives ${ }^{3}$<smiles>O=C(O)CSCC(=O)c1ccc(OO)cc1</smiles>

\footnotetext{
${ }^{2}$ Borzecka, W.; Lavandera, I.; Gotor, V. J. Org. Chem. 2013, 78, 7312.

${ }^{3}$ Lang, C.; Bestgen, S.; Welle, A.; Müller, R.; Roesky, P. W.; Barner-Kowollik, C. Chem. Eur. J. 2015, $21,14728$.
} 
All phenacyl acetic acid derivatives were synthesized following the literature procedure. ${ }^{3}$ Procedure for the synthesis of 2-((2-oxo-2-phenylethyl)thio)acetic acid given as an example. To a cooled solution of $\mathrm{NaOH}(2.0 \mathrm{~g}, 51.43 \mathrm{mmol})$ in $100 \mathrm{~mL} \mathrm{H} \mathrm{H}_{2} \mathrm{O}$, 2-sulfanyl acetic acid $\left(80 \% \mathrm{H}_{2} \mathrm{O}\right.$ solution) (2.3 mL, $25.09 \mathrm{mmol}$ ) was added slowly through a dropping funnel and the reaction mixture was stirred at $0{ }^{\circ} \mathrm{C}$. 2-Bromoacetophenone $(5.0 \mathrm{~g}, 25.11 \mathrm{mmol})$ was dissolved in $40 \mathrm{~mL}$ methanol, added slowly to the reaction mixture, and stirred for $4 \mathrm{~h}$ at ambient temperature. Subsequently, the reaction mixture was poured onto crushed ice and neutralized with Conc. $\mathrm{HCl}$ $(6.0 \mathrm{~mL})$. The precipitate was filtered and purified by recrystallization from $\mathrm{CHCl}_{3}$ to yield 2-((2oxo-2-phenylethyl)thio)acetic acid as a white crystalline solid (4.70 g, $89 \%)$. The spectroscopic data is in agreement with the reported literature data.

(Note - In the cases where precipitate was not formed, the rection mixture was extracted with EtOAc and purified by column chromatography (hexane/EtOAc as an eluent)).

\section{General Procedure for Synthesis of $\beta$-Keto Thioethers ${ }^{4}$}

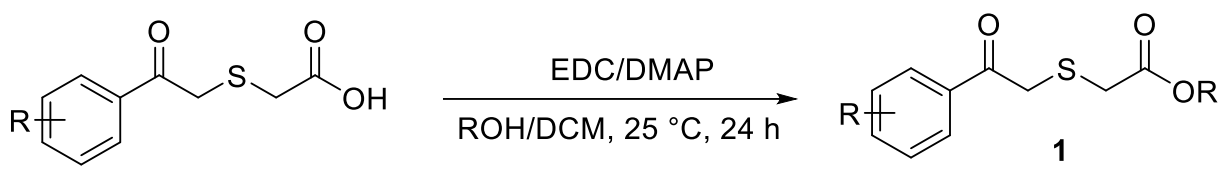

The $\beta$-keto thioethers were synthesized following the literature procedure. ${ }^{4}$ Phenacyl acetic acid derivative $(2.38 \mathrm{mmol})$ was dissolved in $25 \mathrm{~mL}$ of dry DCM. Ethanol (4.76 mmol) and 4(dimethylamino) pyridine (DMAP) (1.19 mmol) were added to the mixture. $N$-(3dimethylaminopropyl)- $N$ '-ethylcarbodiimide hydrochloride (EDC) (4.76 mmol) was dissolved in $13 \mathrm{~mL}$ dry DCM and subsequently added to the solution. The reaction mixture was stirred at ambient temperature for $24 \mathrm{~h}$. The mixture was diluted with $50 \mathrm{~mL}$ of DCM prior to washing in distilled $\mathrm{H}_{2} \mathrm{O}(1 \times 50 \mathrm{~mL})$, saturated $\mathrm{NaHCO}_{3}$ aqueous solution $(2 \times 30 \mathrm{~mL})$ and brine $(1 \times 30 \mathrm{~mL})$. The organic layer was dried over $\mathrm{NaSO}_{4}$, filtered and concentrated under vacuum. The crude product was purified via column chromatography on silica gel eluting with EtOAc/pet ether to obtain the product $\mathbf{1}$.

\footnotetext{
${ }^{4}$ Altintas, O.; Glassner, M.; Rodriguez-Emmenegger, C.; Welle, A.; Trouillet, V. Barner-Kowollik, C. Angew. Chem., Int. Ed. 2015, 54, 5777.
} 


\section{General Procedure for Synthesis of $\beta$-Keto Thioamides}<smiles>O=C(O)CSCC(=O)c1ccccc1</smiles>

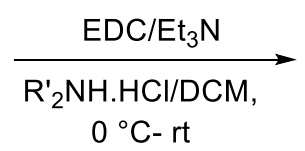<smiles>[R]N([R])C(=O)CSCC(=O)c1cc#[R]cc1</smiles>

To a solution 2-((2-oxo-2-phenyl ethyl)thio)acetic acid $(2.37 \mathrm{mmol})$ and amine hydrochloride $(2.85 \mathrm{mmol})$ in DCM $(7.0 \mathrm{~mL})$ was added $N$-(3-dimethylaminopropyl)- $N^{\prime}-$ ethylcarbodiimide hydrochloride (EDC) $(2.85 \mathrm{mmol})$ in a single batch at $0{ }^{\circ} \mathrm{C}$ under nitrogen. Then $\mathrm{Et}_{3} \mathrm{~N}$ (4.74 mmol) was added dropwise over $10 \mathrm{~min}$. The mixture was allowed to warm to room temperature and stirred overnight. Then the mixture was diluted with $30 \mathrm{~mL}$ of DCM prior to washing in distilled water $(1 \times 50 \mathrm{~mL})$, saturated $\mathrm{NaHCO}_{3}$ aqueous solution $(2 \times 30 \mathrm{~mL})$ and brine $(1 \times 30 \mathrm{~mL})$. The organic layer was dried over $\mathrm{NaSO}_{4}$ filtered and concentrated under vacuum. The crude product was purified via column chromatography on silica gel eluting with EtOAc/pet ether to obtain the product 1.

\section{General Procedure for the Synthesis of Functionalized Enol Ethers}
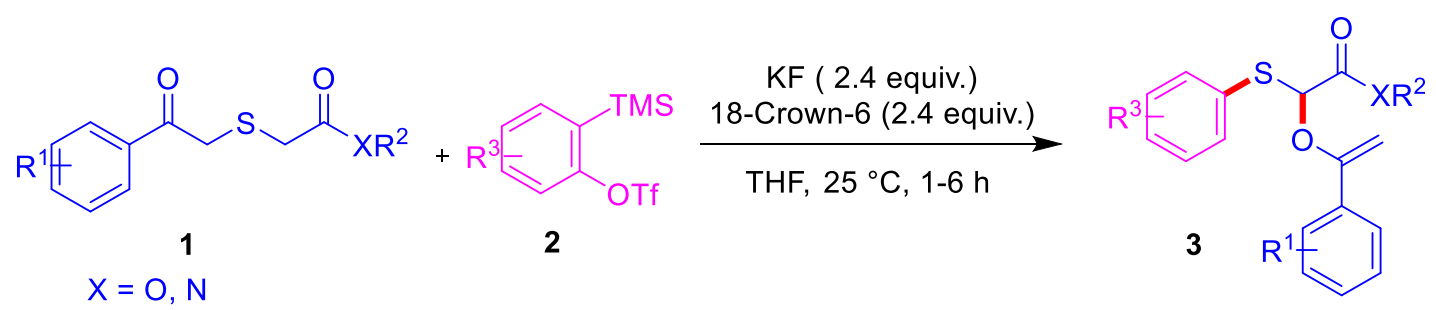

To a flame-dried screw-capped test tube equipped with a magnetic stir bar was added the 18-crown-6 (0.317 g, $1.20 \mathrm{mmol})$ and $\mathrm{KF}(0.070 \mathrm{~g}, 1.20 \mathrm{mmol})$ inside the glove-box. The mixture was dissolved in $2.0 \mathrm{~mL}$ of THF outside the glove-box under nitrogen. To this mixture was added the thioether $1(0.5 \mathrm{mmol})$ (note - solid thioethers were added prior to THF addition) and 2(trimethylsilyl)phenyl trifluoromethanesulfonate $2(0.179 \mathrm{~g}, 146 \mu \mathrm{L}, 0.60 \mathrm{mmol})$. Then the reaction mixture was stirred at $25{ }^{\circ} \mathrm{C}$ for indicated time. After completion of the reaction time (TLC monitoring), the reaction was stopped, the solvent was evaporated and the crude residue preadsorbed on silica gel and purified by flash column chromatography on silica gel (using Pet.etherEtOAc as the eluent) to afford the corresponding enol ether derivatives $\mathbf{3}$ in moderate to good yield. 
Procedure for the Synthesis of 3 a in 2.0 mmol Scale<smiles>CCOC(=O)CSCC(=O)c1ccccc1</smiles>

$1 \mathrm{a}$

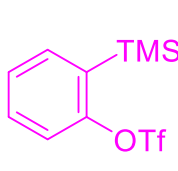

2a

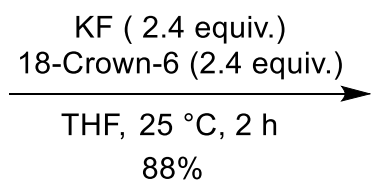

$88 \%$

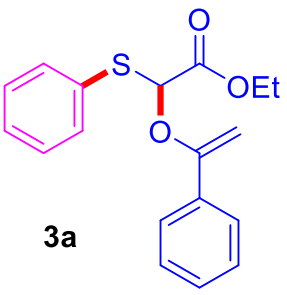

To a flame-dried screw-capped test tube equipped with a magnetic stir bar was added the 18-crown-6 (1.3 g, $4.8 \mathrm{mmol})$ and KF (0.278 g, $4.8 \mathrm{mmol})$ inside the glove-box. The mixture was dissolved in $8.0 \mathrm{~mL}$ of THF outside the glove-box under nitrogen. To this mixture was added ethyl 2-((2-oxo-2-phenylethyl)thio)acetate $1 \mathrm{a} \quad(2.0 \quad \mathrm{mmol})$ and $2-$ (trimethylsilyl)phenyl trifluoromethanesulfonate $2 \mathrm{a}(0.716 \mathrm{~g}, 583 \mu \mathrm{L}, 2.4 \mathrm{mmol})$. Then the reaction mixture was stirred at $25{ }^{\circ} \mathrm{C}$ for $2 \mathrm{~h}$. Subsequently, the reaction was stopped, the solvent was evaporated and the crude residue pre-adsorbed on silica gel and purified by flash column chromatography (Pet.ether/EtOAc $=97 / 03)$ on silica gel to afford the ethyl-2-(phenylthio)-2-((1-phenylvinyl)oxy)acetate 3a as a colourless oil (0.692 g, 88\% yield).

\section{Mechanistic Experiments}

a) Cross-over experiments: Experiments to confirm oxa-[2,3] sigmatropic rearrangement and to rule out the Pummerer-type rearrangement pathway

\section{Cross-over using $1 \mathrm{~h}$ and $1 \mathrm{u}$ as substrates}<smiles>CCOC(=O)CSCC(=O)c1ccc([N+](=O)[O-])cc1</smiles><smiles>CS(=O)(=O)c1ccccc1O</smiles><smiles>CO[C+](CSCC(=O)c1ccccc1)OC</smiles>

$\mathrm{KF}$ (2.4 equiv) $\stackrel{18-C r o w n-6(2.4 \text { equiv) }}{\longrightarrow}$

THF, $25^{\circ} \mathrm{C}, 2 \mathrm{~h}$

Only two products formed<smiles>C=C(OC(Sc1ccccc1)C(=O)OCC)c1ccc([N+](=O)[O-])cc1</smiles><smiles>C=C(OC(Sc1ccccc1)C(C)=O)C(=O)OC(=O)C(=O)OC(=O)c1ccc([N+](=O)[O-])cc1</smiles>

To a flame-dried screw-capped test tube equipped with a magnetic stir bar was added the 18-crown-6 (0.317 g, $1.20 \mathrm{mmol}), \mathrm{KF}(0.070 \mathrm{~g}, 1.20 \mathrm{mmol})$ inside the glove-box. The mixture was 
dissolved in $2.0 \mathrm{~mL}$ of THF outside the glove-box under nitrogen. To this mixture was added ethyl 2-((2-(4-nitrophenyl)-2-oxoethyl)thio)acetate $\mathbf{1 h}(0.071 \mathrm{~g}, 0.25 \mathrm{mmol})$, methyl 2-((2-oxo-2phenylethyl)thio)acetate $\mathbf{1 t}(0.056 \mathrm{~g}, 0.25 \mathrm{mmol})$ and 2- (trimethylsilyl)phenyl trifluoromethane sulfonate 2a $(0.179 \mathrm{~g}, 146 \mu \mathrm{L}, 0.60 \mathrm{mmol})$. Then the reaction mixture was stirred at $25{ }^{\circ} \mathrm{C}$ for $2 \mathrm{~h}$. After completion of indicated time, reaction mixture was diluted with $\mathrm{CH}_{2} \mathrm{Cl}_{2}(2.0 \mathrm{~mL})$ and filtered through a short pad of silica gel and eluted with $\mathrm{CH}_{2} \mathrm{Cl}_{2}(15.0 \mathrm{~mL})$. The solvent was evaporated to obtain the crude product, which was analyzed using ${ }^{1} \mathrm{H}$ NMR using $\mathrm{CH}_{2} \mathrm{Br}_{2}$ (18 $\left.\mu \mathrm{L}, 0.25 \mathrm{mmol}\right)$ as the internal standard. ${ }^{1} \mathrm{H}$ NMR analysis confirmed the formation of two products ethyl 2-((1-(4nitrophenyl)vinyl)oxy)-2-(phenylthio)acetate $\mathbf{3 h}$ (73\% yield) and methyl 2-(phenylthio)-2-((1phenylvinyl)oxy)acetate 3u (92\% yield). Cross-over products 3ax and 3ay were not detected.

\section{${ }^{1}$ H-NMR Spectrum of ethyl-2-((1-(4-nitrophenyl)vinyl)oxy)-2-(phenylthio)acetate (3h)}

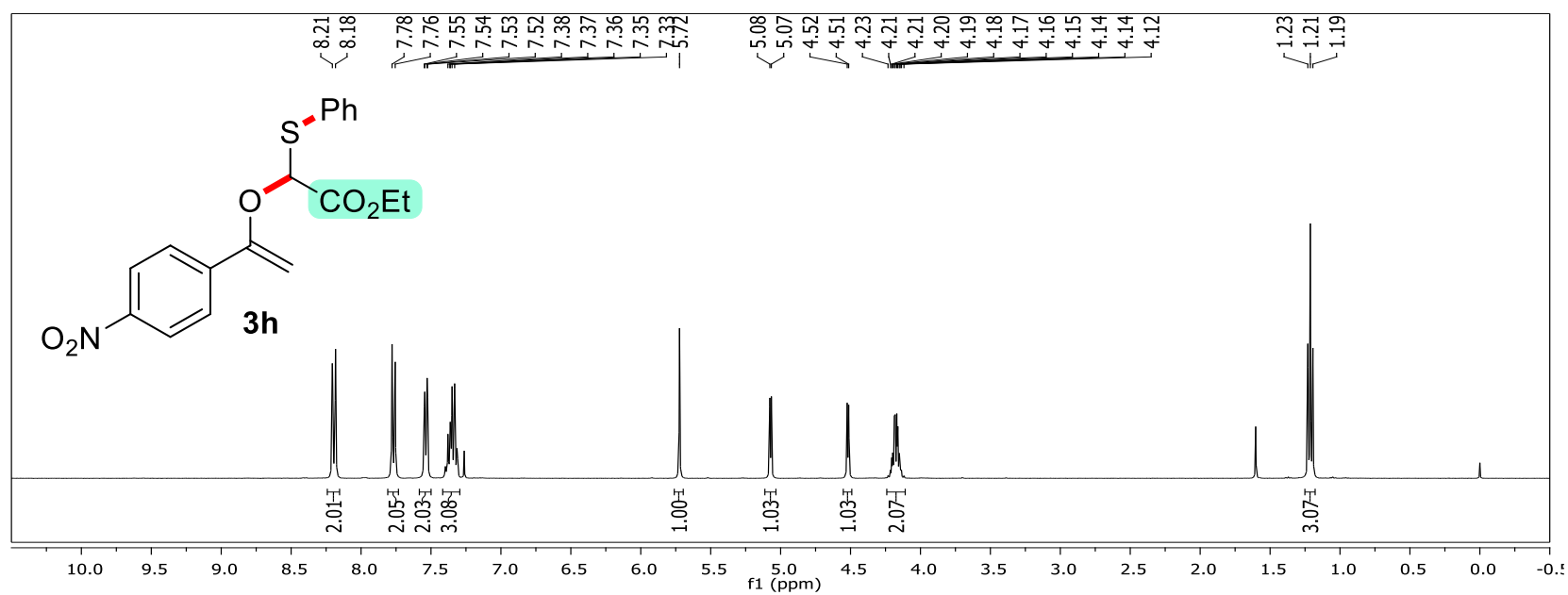

${ }^{1}$ H-NMR Spectrum of methyl-2-(phenylthio)-2-((1-phenylvinyl)oxy)acetate (3u)

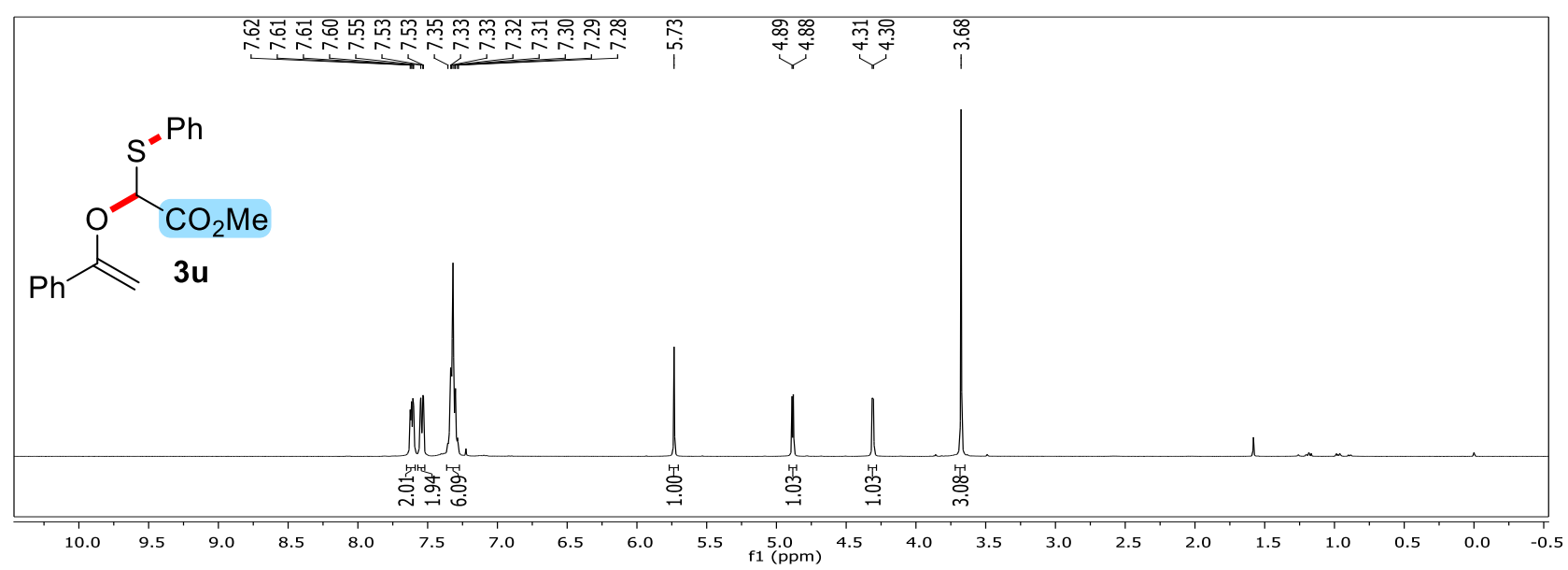


${ }^{1} \mathrm{H}-\mathrm{NMR}$ of Crude Reaction Mixture

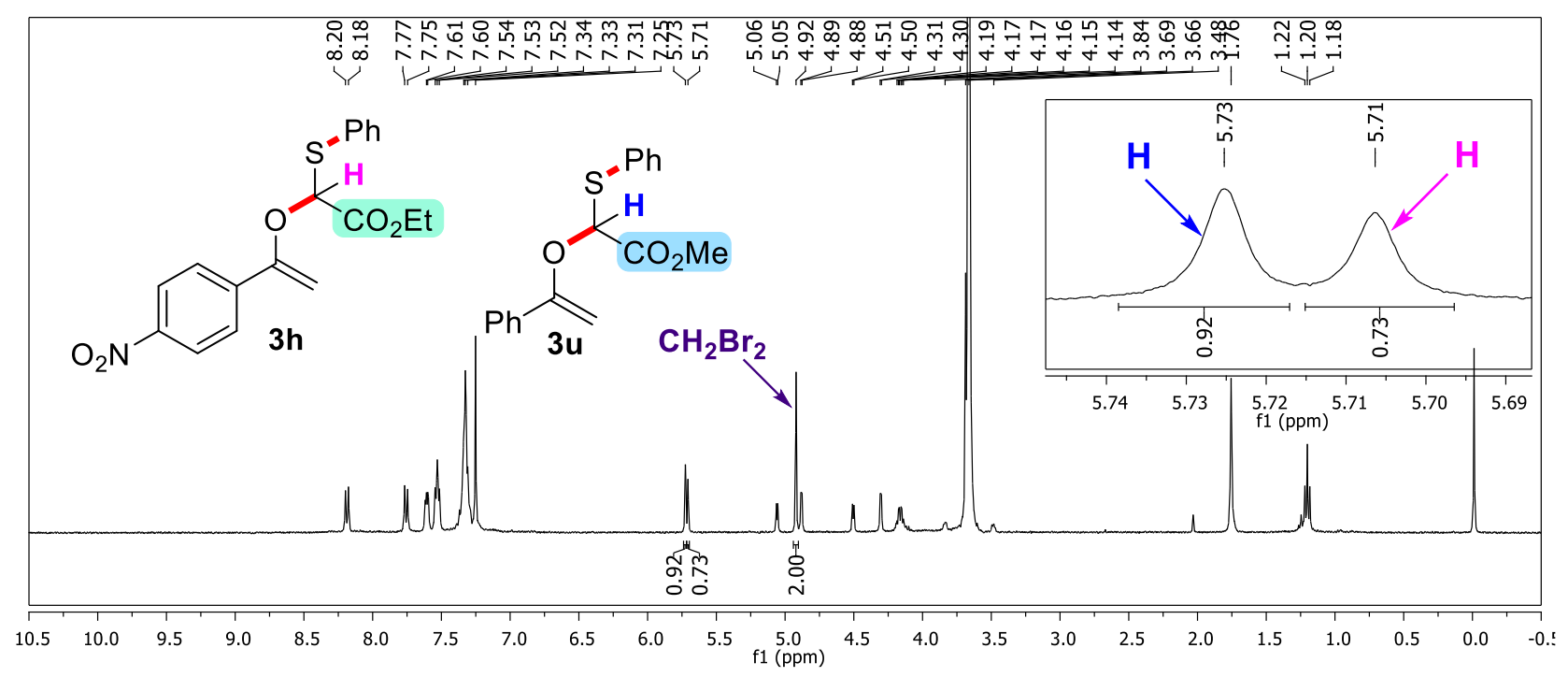

Cross-over using $1 b$ and $1 \mathrm{u}$ as substrates<smiles>CCOC(=O)CSCC(=O)c1ccc(OC)cc1</smiles><smiles>CS(=O)(=O)c1ccccc1Br</smiles><smiles>CO[C+](C)CSCC(=O)c1ccccc1</smiles>

KF (2.4 equiv) $\stackrel{\text { 18-Crown-6 (2.4 equiv) }}{\stackrel{\mathrm{THF}, 25^{\circ} \mathrm{C}, 2 \mathrm{~h}}{\longrightarrow}}$

Only two products formed<smiles>C=C(OC(Sc1ccccc1)C(=O)OCC)c1ccc(OC)cc1</smiles>

3b $(88 \%)$<smiles>C=C(OC(=O)C(=O)OC(Sc1ccccc1)C(=O)OCC)c1ccc(OC)cc1</smiles>

To a flame-dried screw-capped test tube equipped with a magnetic stir bar was added the 18-crown-6 (0.317 g, $1.20 \mathrm{mmol}), \mathrm{KF}(0.070 \mathrm{~g}, 1.20 \mathrm{mmol})$ inside the glove-box. The mixture was dissolved in $2.0 \mathrm{~mL}$ of THF outside the glove-box under nitrogen. To this mixture was added ethyl 2-((2-(4-methoxyphenyl)-2-oxoethyl)thio)acetate $\mathbf{1 b}(0.067 \mathrm{~g}, 0.25 \mathrm{mmol})$, methyl 2-((2-oxo-2phenylethyl)thio)acetate $1 \mathbf{u}(0.056 \mathrm{~g}, 0.25 \mathrm{mmol})$ and 2- (trimethylsilyl)phenyl trifluoromethane sulfonate $2 \mathbf{a}(0.179 \mathrm{~g}, 146 \mu \mathrm{L}, 0.60 \mathrm{mmol})$. Then the reaction mixture was stirred at $25^{\circ} \mathrm{C}$ for $2 \mathrm{~h}$. After completion of indicated time, the reaction mixture was diluted with $\mathrm{CH}_{2} \mathrm{Cl}_{2}(2.0 \mathrm{~mL})$ and filtered through a short pad of silica gel and eluted with $\mathrm{CH}_{2} \mathrm{Cl}_{2}(15.0 \mathrm{~mL})$. The solvent was evaporated to obtain the crude product, which was analysed using ${ }^{1} \mathrm{H} \mathrm{NMR}$ using $\mathrm{CH}_{2} \mathrm{Br}_{2}(18 \mu \mathrm{L}$, $0.25 \mathrm{mmol}$ ) as the internal standard. ${ }^{1} \mathrm{H}$ NMR analysis confirmed the formation of two products 
ethyl 2-((1-(4-methoxyphenyl)vinyl)oxy)-2-(phenylthio)acetate $3 \mathbf{b}$ (88\% yield) and methyl 2(phenylthio)-2-((1-phenylvinyl)oxy)acetate 3u (90\% yield). Cross-over products 3az and 3ay were not detected.

${ }^{1}$ H-NMR Spectrum of ethyl 2-((1-(4-methoxyphenyl)vinyl)oxy)-2-(phenylthio)acetate (3b)

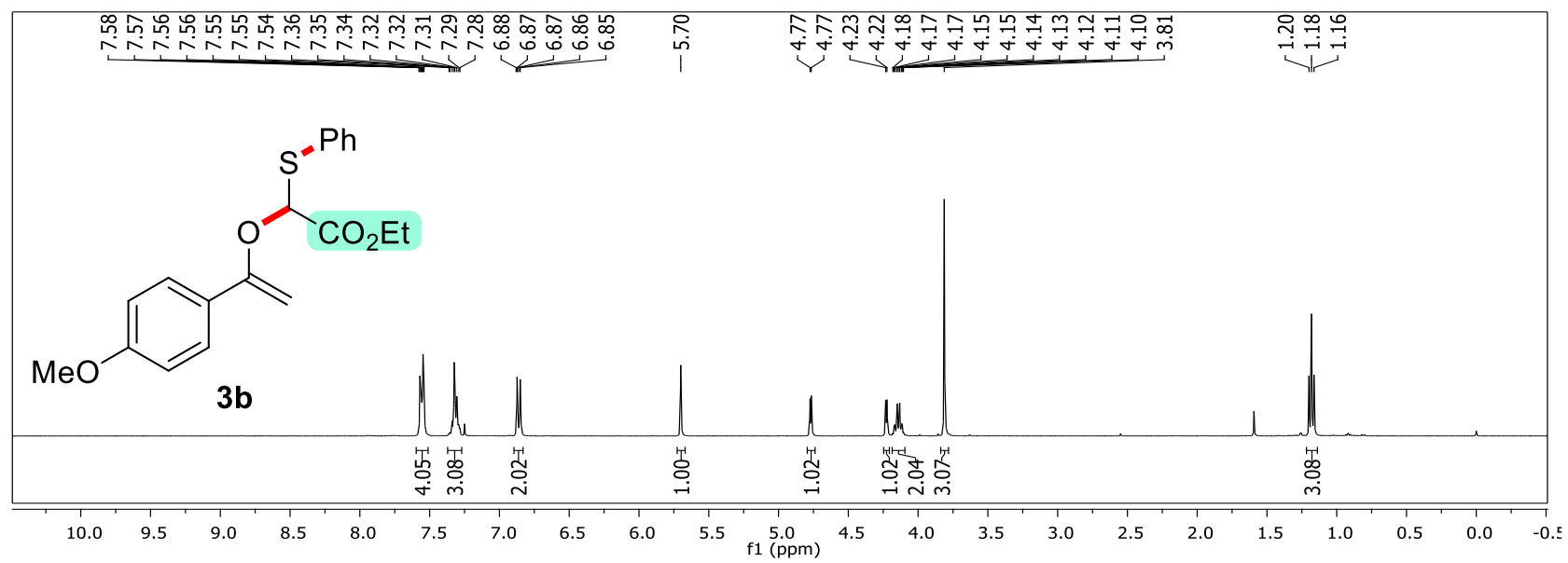

${ }^{1}$ H-NMR Spectrum of methyl-2-(phenylthio)-2-((1-phenylvinyl)oxy)acetate (3u)

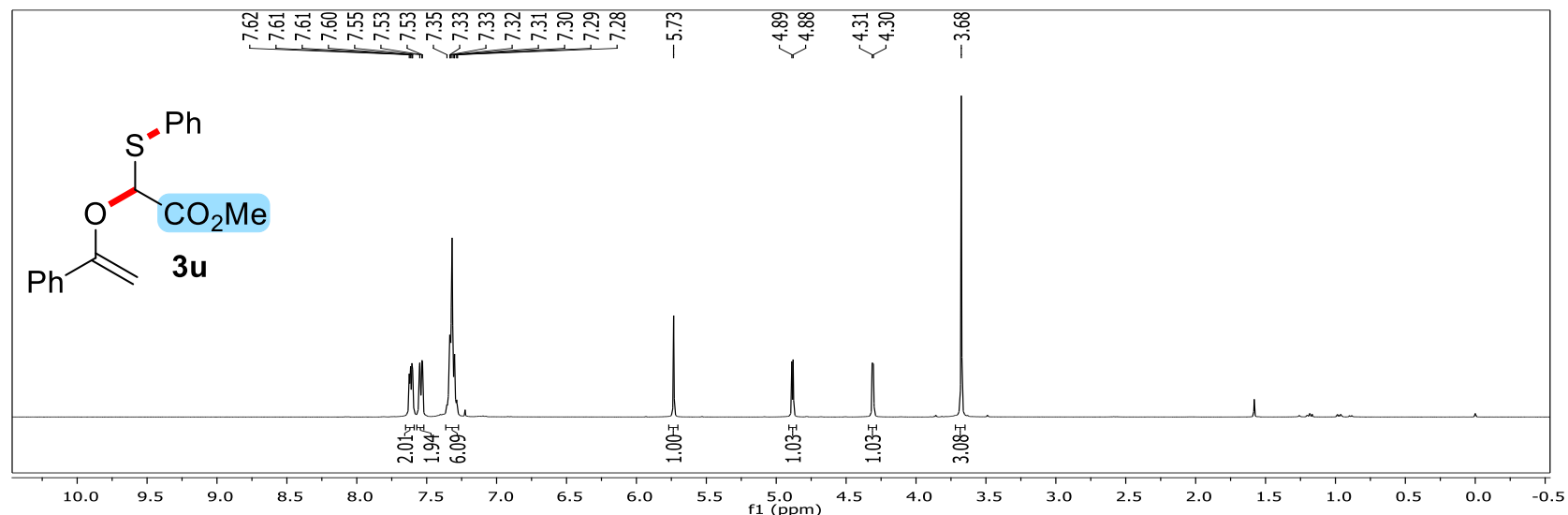

${ }^{1}$ H-NMR of Crude Reaction Mixture

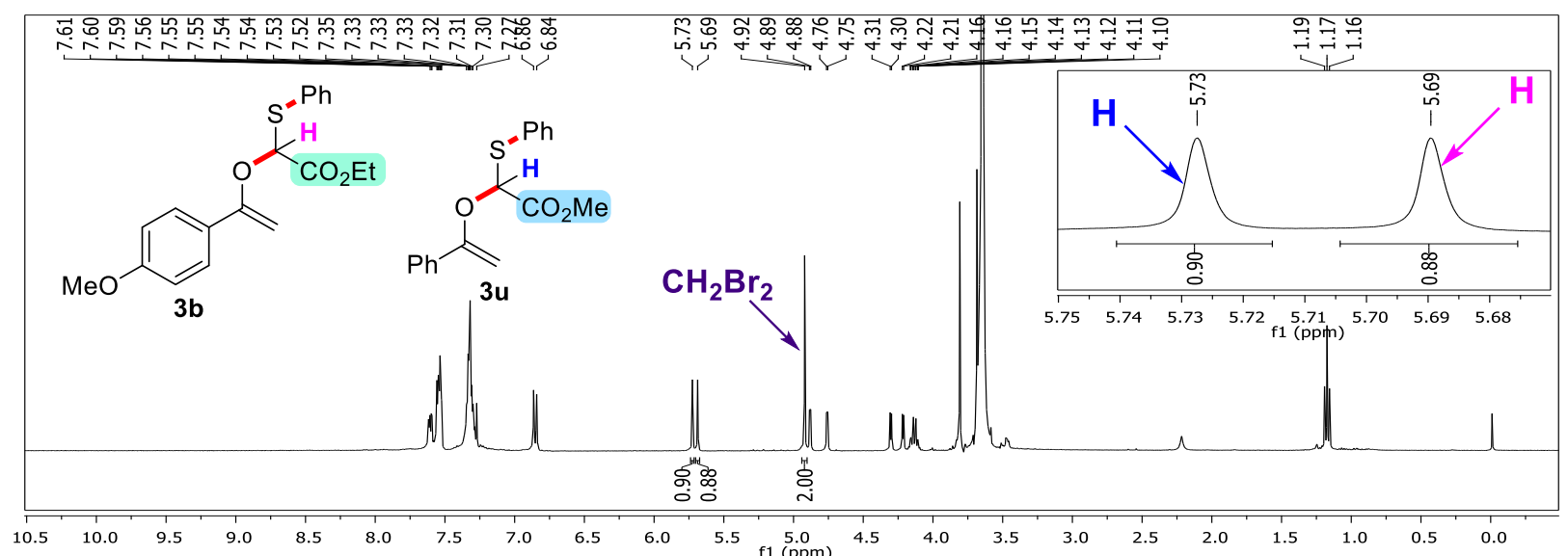


The absence of the cross-over products is an indication that the reaction proceeds via oxa-[2,3] sigmatropic rearrangement not via the Pummerer-type rearrangement pathway (Scheme $4 B$ of the manuscript).

\section{b) Reaction in the Presence of an External Nucleophile}

\section{Diethyl malonate as an external nucleophile}<smiles>CCOC(=O)CSCC(=O)c1ccccc1</smiles>

$1 \mathrm{a}$<smiles>CCOC(=O)CC(=O)OCC</smiles>

KF (2.4 equiv)<smiles>CS(=O)(=O)c1ccccc1O</smiles>

2a 18-C-6 (2.4 equiv)

THF, $25^{\circ} \mathrm{C}, 1 \mathrm{~h}$<smiles>O=C(OC(SSc1ccccc1)C(=O)[O-])c1ccccc1</smiles><smiles>CCOC(=O)c1ccccc1C(=O)OCC</smiles>

Trapping with external nucleophile<smiles>CCOC(=O)C(Sc1ccccc1)C(C(=O)OCC)C(=O)OCC</smiles>

6 (not formed)

To a flame-dried screw-capped test tube equipped with a magnetic stir bar was added the 18-crown-6 (0.158 g, $0.6 \mathrm{mmol}), \mathrm{KF}(0.035 \mathrm{~g}, 0.6 \mathrm{mmol})$ inside the glove-box. The mixture was dissolved in $1.0 \mathrm{~mL}$ of THF outside the glove-box under nitrogen. To this mixture was added ethyl 2-((2-oxo-2-phenylethyl)thio)acetate $1 \mathrm{a}(0.060 \mathrm{~g}, 0.25 \mathrm{mmol})$, diethyl malonate $4(0.040 \mathrm{~g}, 38 \mu \mathrm{L}$, $0.25 \mathrm{mmol})$ and 2- (trimethylsilyl)phenyl trifluoromethanesulfonate 2a $(0.090 \mathrm{~g}, 73 \mu \mathrm{L}, 0.30$ $\mathrm{mmol}$ ). Then, the reaction mixture was stirred at $25^{\circ} \mathrm{C}$ for $1 \mathrm{~h}$. After completion of indicated time, the reaction mixture was diluted with $\mathrm{CH}_{2} \mathrm{Cl}_{2}(1.0 \mathrm{~mL})$ and filtered through a short pad of silica gel and eluted with $\mathrm{CH}_{2} \mathrm{Cl}_{2}(8.0 \mathrm{~mL})$. The solvent was evaporated to obtain the crude product, which was analysed using ${ }^{1} \mathrm{H}$ NMR using $\mathrm{CH}_{2} \mathrm{Br}_{2}(18 \mu \mathrm{L}, 0.25 \mathrm{mmol})$ as the internal standard. ${ }^{1} \mathrm{H}$ NMR analysis confirmed the formation of rearranged product ethyl-2-(phenylthio)-2-((1phenylvinyl)oxy)acetate 3a (94\% yield), and the insertion product 5 (9\% yield). Nucleophile trapped product $\mathbf{6}$ was not observed (to trap the intermediate III). 
${ }^{1}$ H-NMR Spectrum of ethyl-2-(phenylthio)-2-((1-phenylvinyl)oxy)acetate (3a)

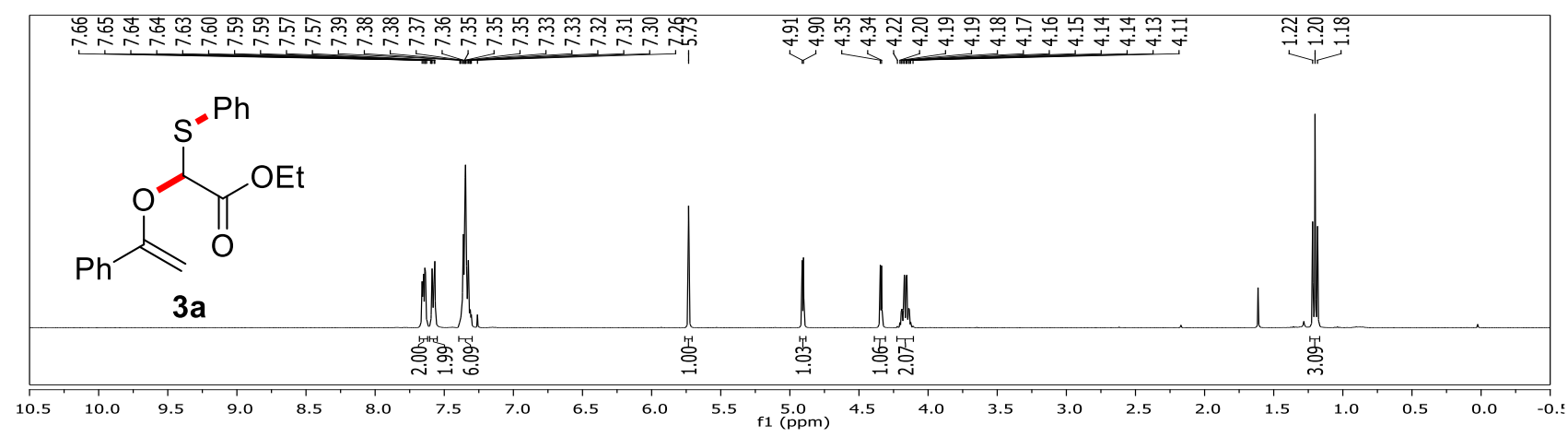

${ }^{1} \mathrm{H}-\mathrm{NMR}$ Spectrum of reaction mixture after $1 \mathrm{~h}$

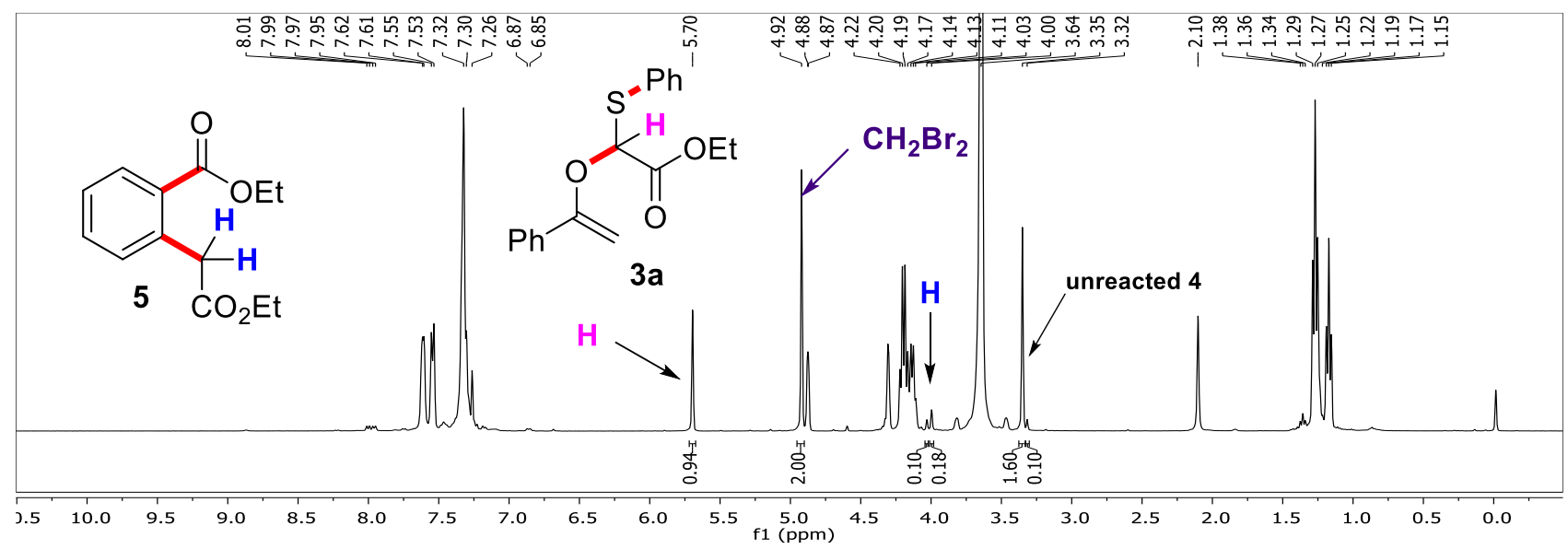

Ethyl acetoacetate as an external nucleophile<smiles>CCOC(=O)CSCC(=O)c1ccccc1</smiles><smiles>CCOC(=O)CC(C)=O</smiles>

15<smiles>CS(=O)(=O)c1ccccc1O</smiles>

$2 a$
KF (2.4 equiv) 18-C-6 (2.4 equiv)

THF, $25^{\circ} \mathrm{C}, 1 \mathrm{~h}$

Trapping with external nucleophile<smiles>C=C(OC(Sc1ccccc1)C(=O)OCC)c1ccccc1</smiles><smiles>CCOC(=O)Cc1ccccc1C(C)=O</smiles><smiles>CCOC(=O)C(SP)C(C(=O)OCC)C(C(C)=O)C(=O)O</smiles>

To a flame-dried screw-capped test tube equipped with a magnetic stir bar was added the 18-crown-6 (0.158 g, $0.6 \mathrm{mmol}), \mathrm{KF}(0.035 \mathrm{~g}, 0.6 \mathrm{mmol})$ inside the glove-box. The mixture was dissolved in $1.0 \mathrm{~mL}$ of THF outside the glove-box under nitrogen. To this mixture was added ethyl 2-((2-oxo-2-phenylethyl)thio)acetate $1 \mathbf{a}(0.060 \mathrm{~g}, 0.25 \mathrm{mmol})$, ethyl 3-oxobutanoate 15 (0.033 g, $32 \mu \mathrm{L}, 0.25 \mathrm{mmol})$ and 2- (trimethylsilyl)phenyl trifluoromethanesulfonate $2 \mathbf{a}(0.090 \mathrm{~g}, 73 \mu \mathrm{L}$, 
$0.30 \mathrm{mmol})$. Then, the reaction mixture was stirred at $25^{\circ} \mathrm{C}$ for $1 \mathrm{~h}$. After completion of indicated time reaction mixture was diluted with $\mathrm{CH}_{2} \mathrm{Cl}_{2}(1.0 \mathrm{~mL})$ and filtered through a short pad of silica gel and eluted with $\mathrm{CH}_{2} \mathrm{Cl}_{2}(8.0 \mathrm{~mL})$. The solvent was evaporated to obtain the crude product, which was analysed using ${ }^{1} \mathrm{H}$ NMR using $\mathrm{CH}_{2} \mathrm{Br}_{2}(18 \mu \mathrm{L}, 0.25 \mathrm{mmol})$ as the internal standard. ${ }^{1} \mathrm{H}$ NMR analysis confirmed the formation of rearranged products ethyl-2-(phenylthio)-2-((1phenylvinyl)oxy)acetate 3a (56\% yield), and insertion product $\mathbf{1 6}$ (45\% yield). Nucleophile trapped product 17 was not observed.

\section{${ }^{1}$ H-NMR Spectrum of ethyl-2-(phenylthio)-2-((1-phenylvinyl)oxy)acetate (3a)}

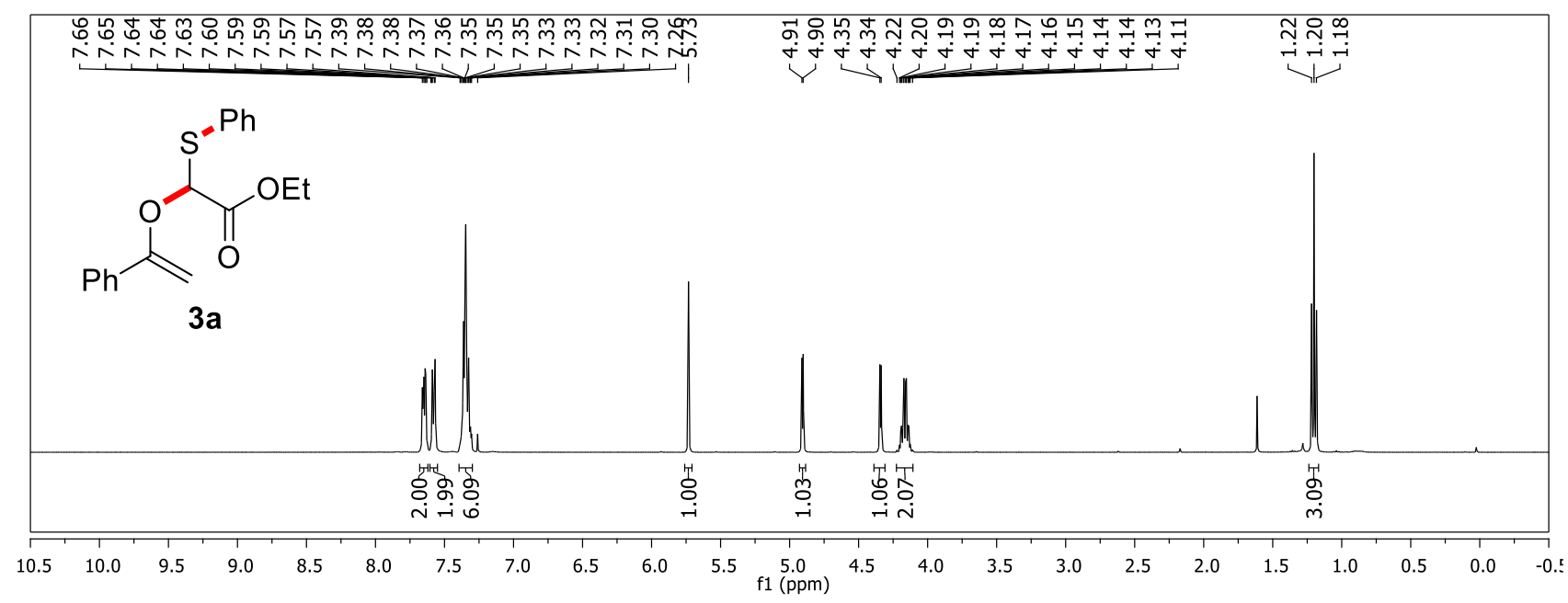

\section{${ }^{1} \mathrm{H}-\mathrm{NMR}$ Spectrum of reaction mixture after $1 \mathrm{~h}$}

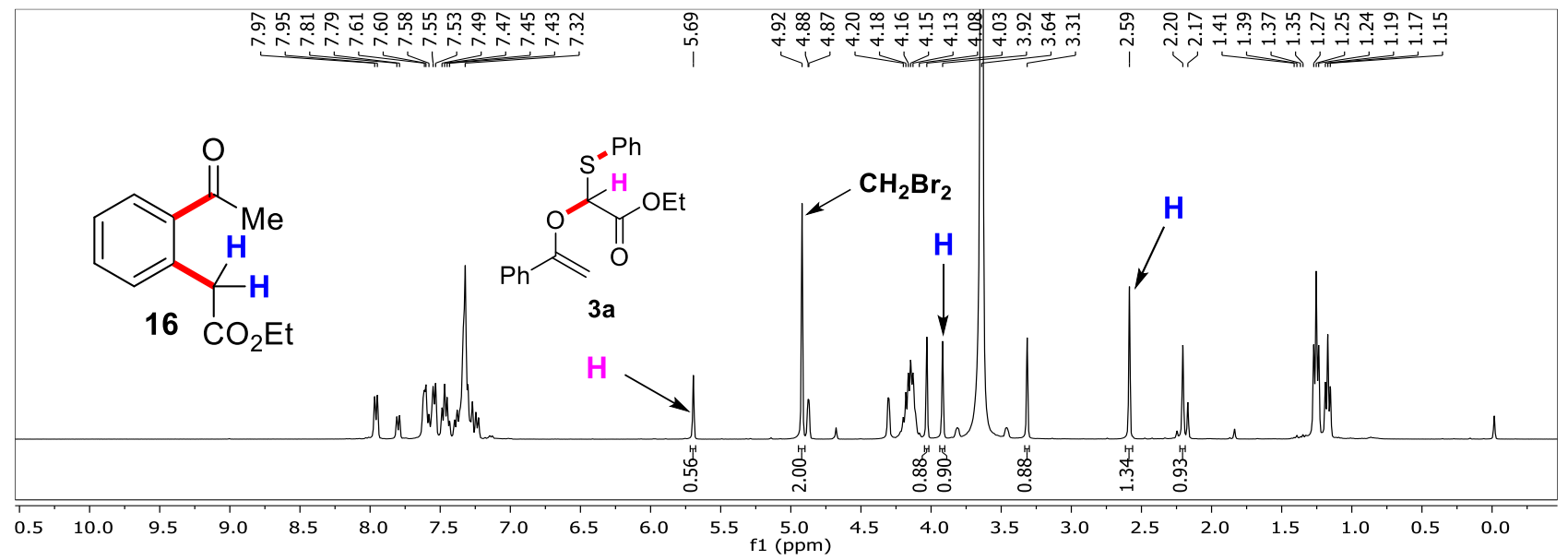

This result tends to indicate that the present rearrangement is intramolecular (Scheme $4(C))$ and absence of nucleophile trapped product confirmed that reaction proceed via oxa-[2,3] sigmatropic rearrangement not via Pummerer-type rearrangement pathway. 


\section{c) Competition experiments}

\section{Competition between oxa-[2,3] and Stevens [2,3]}<smiles>C=CCSCC(=O)OCC</smiles><smiles>CS(=O)(=O)c1ccccc1O</smiles>

$\mathrm{KF}(2.4$ equiv) 18-C-6 (2.4 equiv)

THF, $25^{\circ} \mathrm{C}, 5 \mathrm{~min}$<smiles>C=CCC(SPc1ccccc1)C(=O)OCC</smiles>

To a flame-dried screw-capped test tube equipped with a magnetic stir bar was added the 18-crown-6 (0.158 g, $0.60 \mathrm{mmol}), \mathrm{KF}(0.035 \mathrm{~g}, 0.60 \mathrm{mmol})$ inside the glove-box. The mixture was dissolved in $1.0 \mathrm{~mL}$ of THF outside the glove-box under nitrogen. To this mixture was added ethyl 2-((2-oxo-2-phenylethyl)thio)acetate $1 \mathbf{a}(0.030 \mathrm{~g}, 0.125 \mathrm{mmol})$, ethyl 2-(allylthio)acetate 7 (0.020 g, $0.125 \mathrm{mmol})$ and 2- (trimethylsilyl)phenyl trifluoromethanesulfonate $\mathbf{2 a}(0.090 \mathrm{~g}, 73.0 \mu \mathrm{L}, 0.30$ mmol). Then, the reaction mixture was stirred at $25{ }^{\circ} \mathrm{C}$ for $5 \mathrm{~min}$. After completion of indicated time, the reaction mixture was diluted with $\mathrm{CH}_{2} \mathrm{Cl}_{2}(2.0 \mathrm{~mL})$ and filtered through a short pad of silica gel and eluted with $\mathrm{CH}_{2} \mathrm{Cl}_{2}(15.0 \mathrm{~mL})$. The solvent was evaporated to obtain the crude product, which was analysed using ${ }^{1} \mathrm{H}$ NMR using $\mathrm{CH}_{2} \mathrm{Br}_{2}(18 \mu \mathrm{L}, 0.25 \mathrm{mmol})$ as the internal standard. ${ }^{1} \mathrm{H}$ NMR analysis confirmed the formation of two products ethyl-2-(phenylthio)-2-((1phenylvinyl)oxy)acetate 3a (42\% yield) and ethyl-2-(phenylthio)pent-4-enoate $\mathbf{8}$ (44\% yield).

\section{${ }^{1}$ H-NMR Spectrum of ethyl-2-(phenylthio)-2-((1-phenylvinyl)oxy)acetate (3a)}

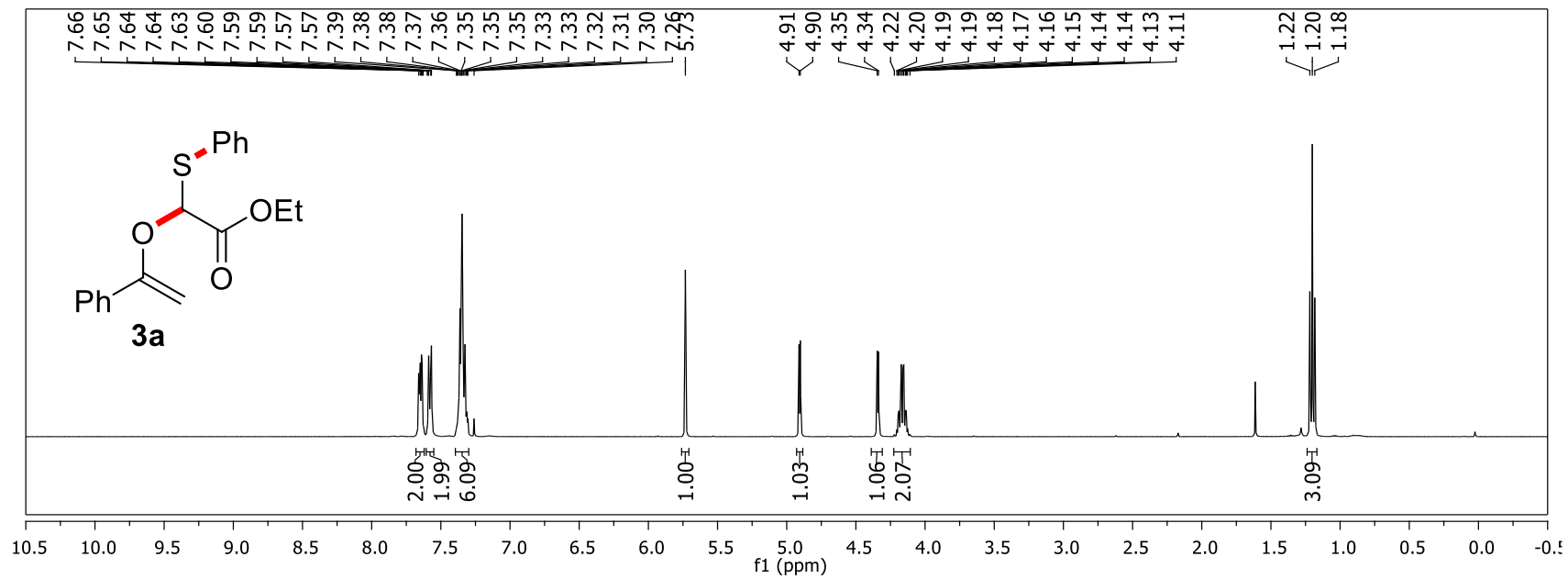

${ }^{5}$ Tan, J.-J.; Zheng, T.-Y.; Xu, K.; Liu, C.-Y. Org. Biomol. Chem. 2017, 15, 4946. 


\section{${ }^{1}$ H-NMR Spectrum of ethyl-2-(phenylthio)pent-4-enoate (8)}

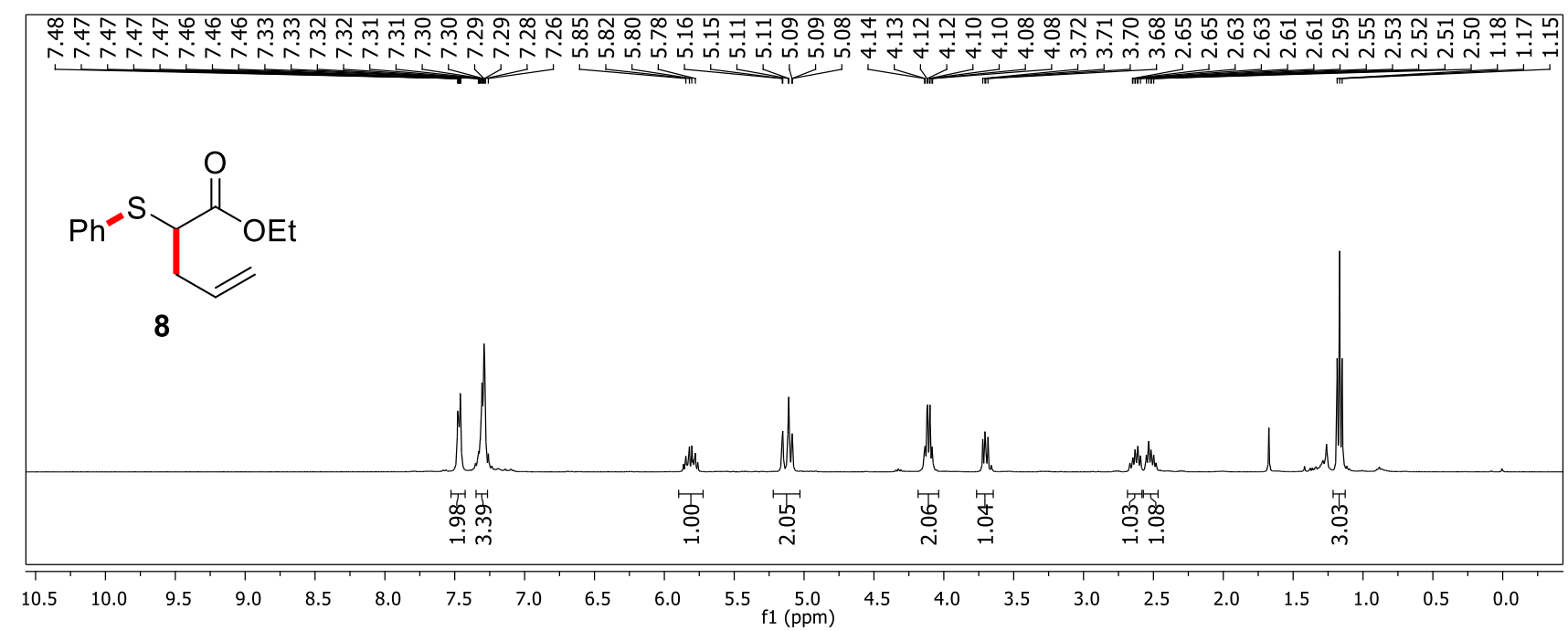

${ }^{1}$ H-NMR Spectrum of reaction mixture after 5 min

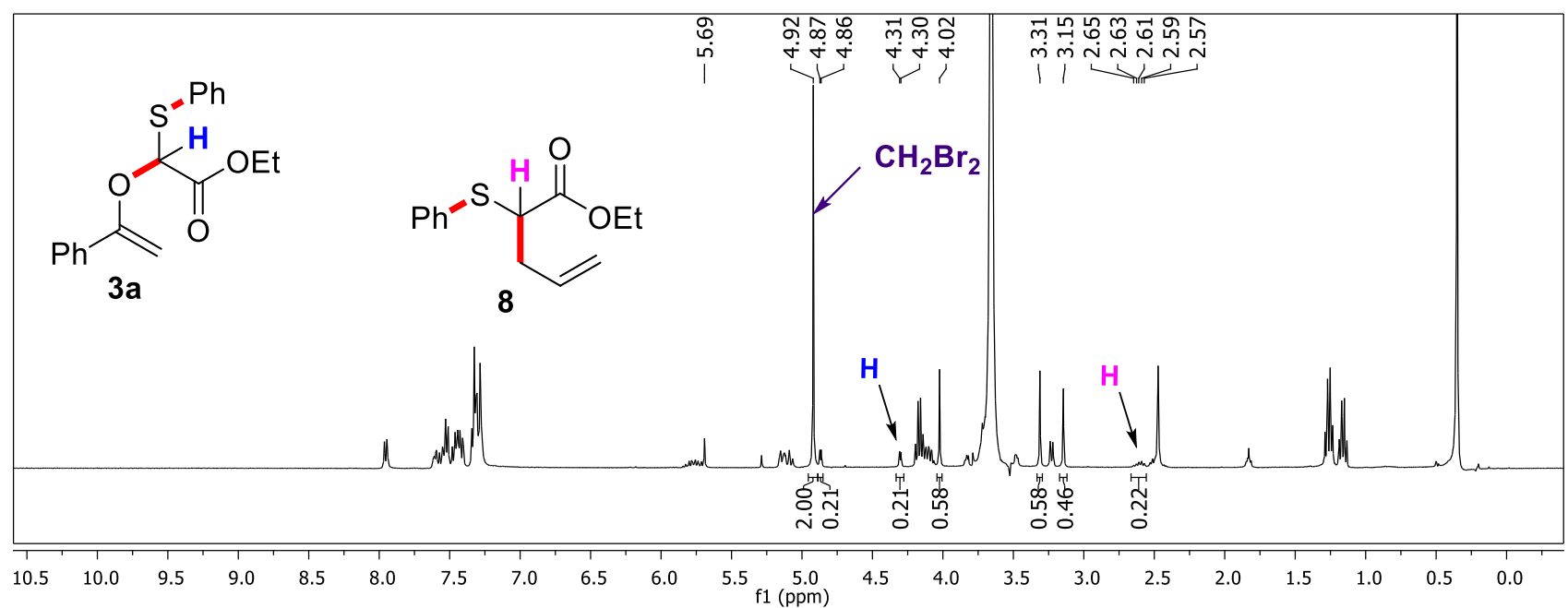

Form the above competition experiment, it is likely that both the Stevens [2,3] rearrangement and oxa-[2,3] sigmatropic rearrangement proceed with comparable rates (product ratio observed 1:1). The initial attack by the sulfide is very likely the product-determining event in both cases.

\section{Competition between two electronically different substrates}

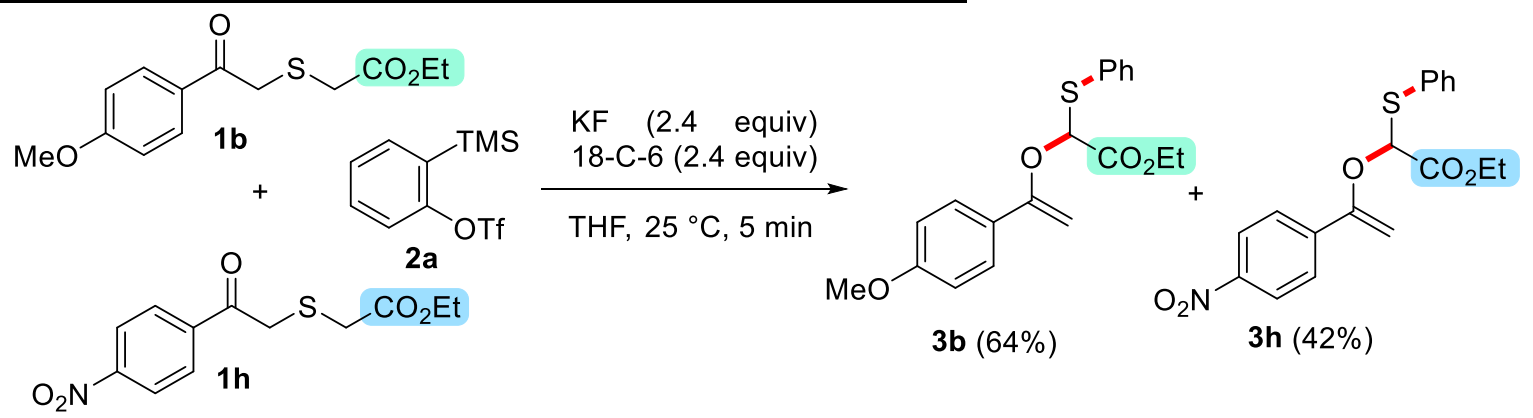


To a flame-dried screw-capped test tube equipped with a magnetic stir bar was added the 18-crown-6 (0.158 g, $0.60 \mathrm{mmol}), \mathrm{KF}(0.035 \mathrm{~g}, 0.60 \mathrm{mmol})$ inside the glove-box. Then ethyl 2((2-(4-nitrophenyl)-2-oxoethyl)thio)acetate $\mathbf{1 h}(0.035 \mathrm{~g}, 0.125 \mathrm{mmol})$ was added outside the glove-box under nitrogen atmosphere. The mixture was dissolved in $1.0 \mathrm{~mL}$ of THF. To this mixture was added ethyl 2-((2-(4-methoxyphenyl)-2-oxoethyl)thio)acetate 1b (0.034 g, 0.125 mmol) and 2- (trimethylsilyl)phenyl trifluoromethanesulfonate $2 \mathbf{a}(0.090 \mathrm{~g}, 73.0 \mu \mathrm{L}, 0.30 \mathrm{mmol})$. Then, the reaction mixture was stirred at $25{ }^{\circ} \mathrm{C}$ for $5 \mathrm{~min}$. After completion of indicated time reaction mixture was diluted with $\mathrm{CH}_{2} \mathrm{Cl}_{2}(2.0 \mathrm{~mL})$ and filtered through a short pad of silica gel and eluted with $\mathrm{CH}_{2} \mathrm{Cl}_{2}(15.0 \mathrm{~mL})$. The solvent was evaporated to obtain the crude product, which was analysed using ${ }^{1} \mathrm{H} \mathrm{NMR}$ using $\mathrm{CH}_{2} \mathrm{Br}_{2}(18 \mu \mathrm{L}, 0.25 \mathrm{mmol})$ as the internal standard. ${ }^{1} \mathrm{H} \mathrm{NMR}$ analysis confirmed the formation of two products ethyl 2-((1-(4-nitrophenyl)vinyl)oxy)-2(phenylthio)acetate $3 \mathbf{h} \quad(42 \%$ yield) and ethyl 2-((1-(4-methoxyphenyl)vinyl)oxy)-2(phenylthio)acetate $\mathbf{3 b}(64 \%$ yield).

\section{${ }^{1}$ H-NMR Spectrum of ethyl-2-((1-(4-nitrophenyl)vinyl)oxy)-2-(phenylthio)acetate (3h)}

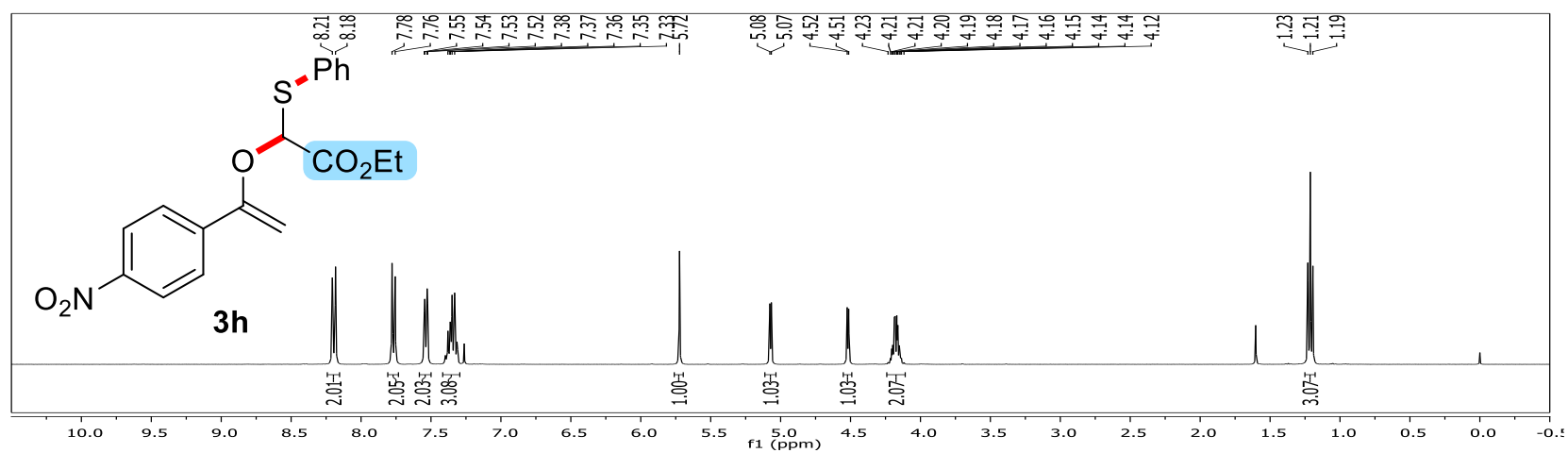

${ }^{1}$ H-NMR Spectrum of ethyl 2-((1-(4-methoxyphenyl)vinyl)oxy)-2-(phenylthio)acetate (3b)

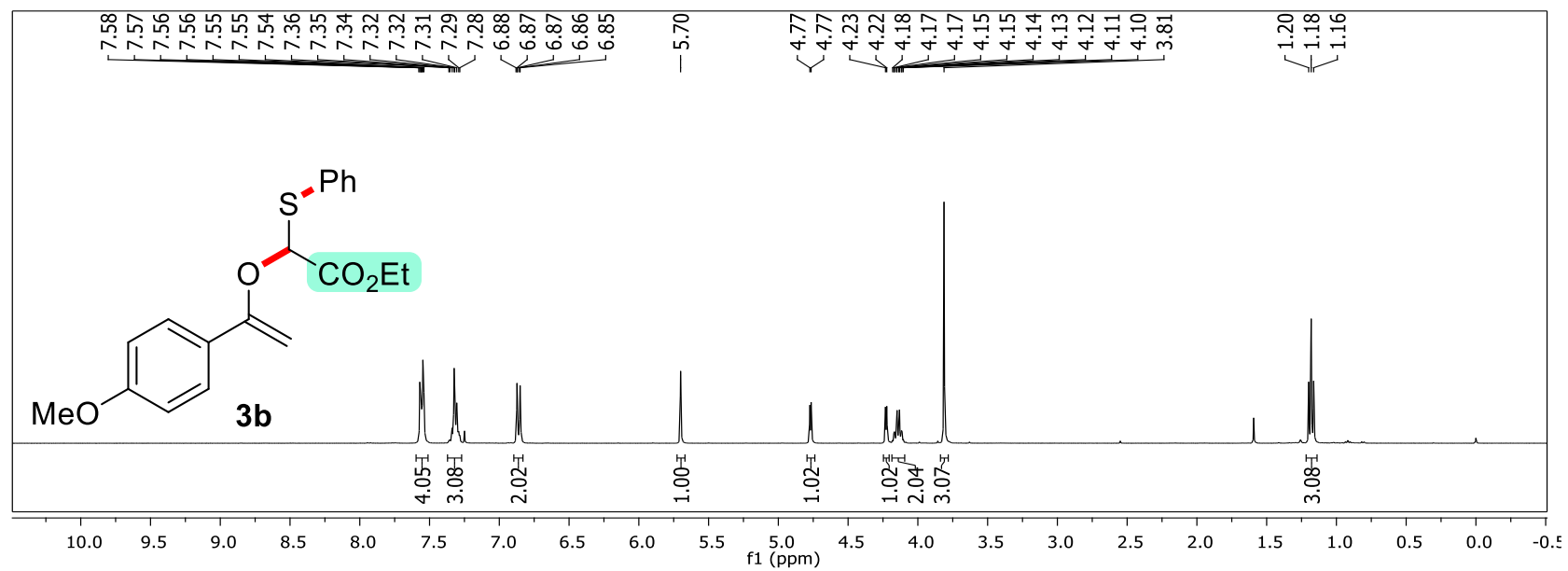


${ }^{1} \mathrm{H}-\mathrm{NMR}$ Spectrum of reaction mixture after $5 \mathrm{~min}$

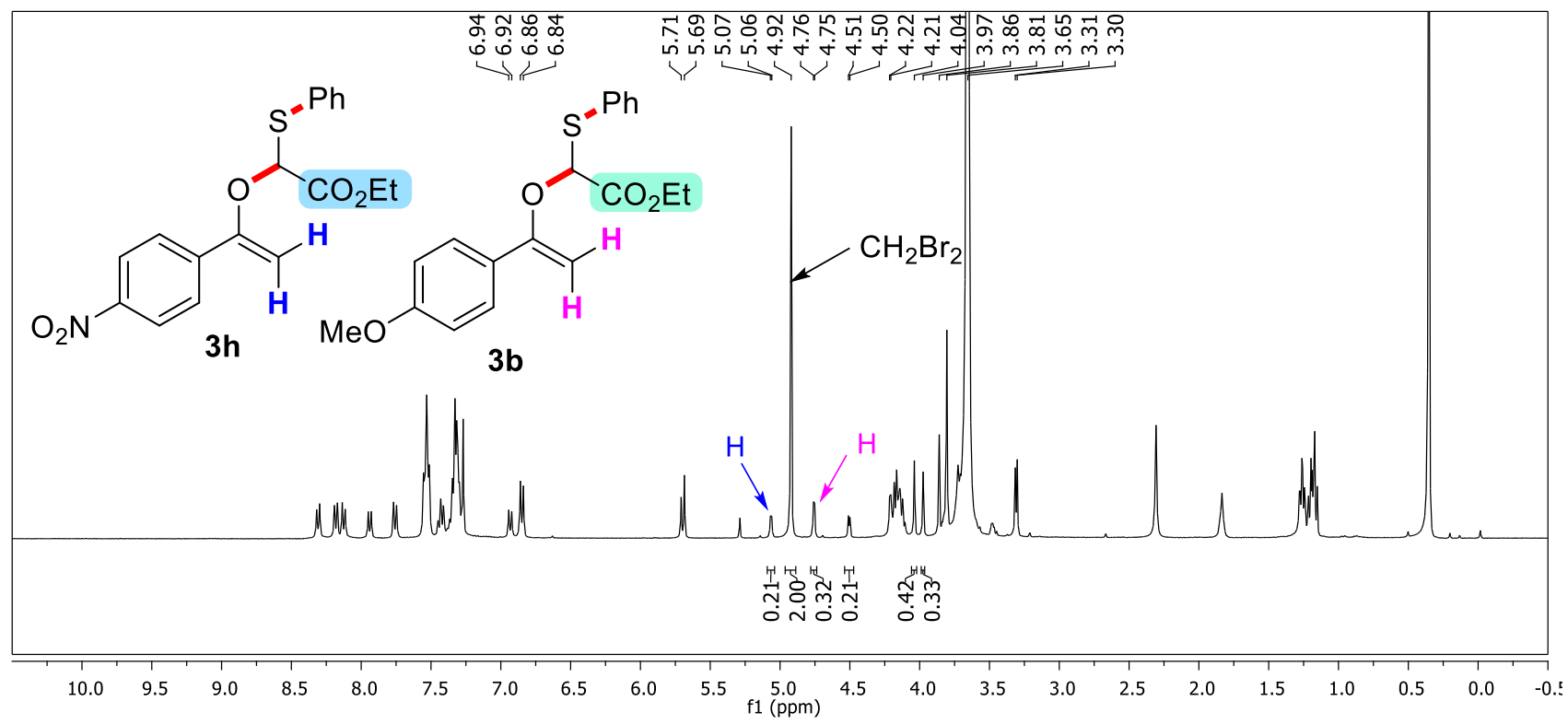

Form the above competition experiment, it indicates that the thioether substrate bearing electronreleasing group at aromatic ring reacts faster than thioether substrate bearing electronwithdrawing group at aromatic ring.

\section{X-Ray Data of 11}

Single crystal of 11 (recrystallized from $\mathrm{CDCl}_{3}$ at $25^{\circ} \mathrm{C}$ ) was mounted and the diffraction data was collected at $296 \mathrm{~K}$ on a Bruker SMART APEX CCD diffractometer using SMART/SAINT software. Intensity data were collected using graphite-monochromatized Mo-Ka radiation $(71.073 \mathrm{pm})$. The structure was solved by direct methods using the SHELX-976 and refined by full-matrix least-squares on F2. Empirical absorption corrections were applied with SADABS. ${ }^{7}$ All Non-hydrogen atoms were refined anisotropically and hydrogen atoms were included in geometric positions. Structure was drawn using Olex-2 and ORTEP-3. The crystallographic refinement parameters are given below:

\section{Table 1 Crystal data and structure refinement for 11}

Identification code

CCDC

Empirical formula

Formula weight

Temperature/K

Crystal system
11

2058553

$\mathrm{C}_{17} \mathrm{H}_{17} \mathrm{NO}_{2} \mathrm{~S}$

299.38

296(2)

orthorhombic

${ }^{6}$ SHELXL, G. M. 2013 Sheldrick, University of Göttingen: Göttingen, Germany, 2014.

${ }^{7}$ Sheldrick, G. M. SADABS, University of Göttingen, Göttingen, Germany, 1999. 
Space group

$\mathrm{a} / \AA$

$\mathrm{b} / \AA$

$\mathrm{c} / \AA$

$\alpha /^{\circ}$

$\beta /{ }^{\circ}$

$\gamma /{ }^{\circ}$

Volume $/ \AA^{3}$

$\mathrm{Z}$

$\rho_{\text {calc }} \mathrm{mg} / \mathrm{mm}^{3}$

$\mathrm{m} / \mathrm{mm}^{-1}$

$\mathrm{F}(000)$

$2 \Theta$ range for data collection

Index ranges

Reflections collected

Independent reflections

Data/restraints/parameters

Goodness-of-fit on $\mathrm{F}^{2}$

Final R indexes [I $>=2 \sigma(\mathrm{I})]$

Final $\mathrm{R}$ indexes [all data]

Largest diff. peak/hole / e $\AA^{-3}$
Pbca

$8.0719(6)$

$10.0338(8)$

$39.512(3)$

90

90

90

$3200.1(4)$

8

1.243

0.206

1264.0

2.062 to $52.56^{\circ}$

$-10 \leq \mathrm{h} \leq 10,-12 \leq \mathrm{k} \leq 12,-49 \leq 1 \leq 49$

52962

$3230[\mathrm{R}($ int $)=0.0445]$

3230/0/195

1.081

$\mathrm{R}_{1}=0.0387, \mathrm{wR}_{2}=0.1067$

$\mathrm{R}_{1}=0.0585, \mathrm{wR}_{2}=0.1294$

$0.18 /-0.20$

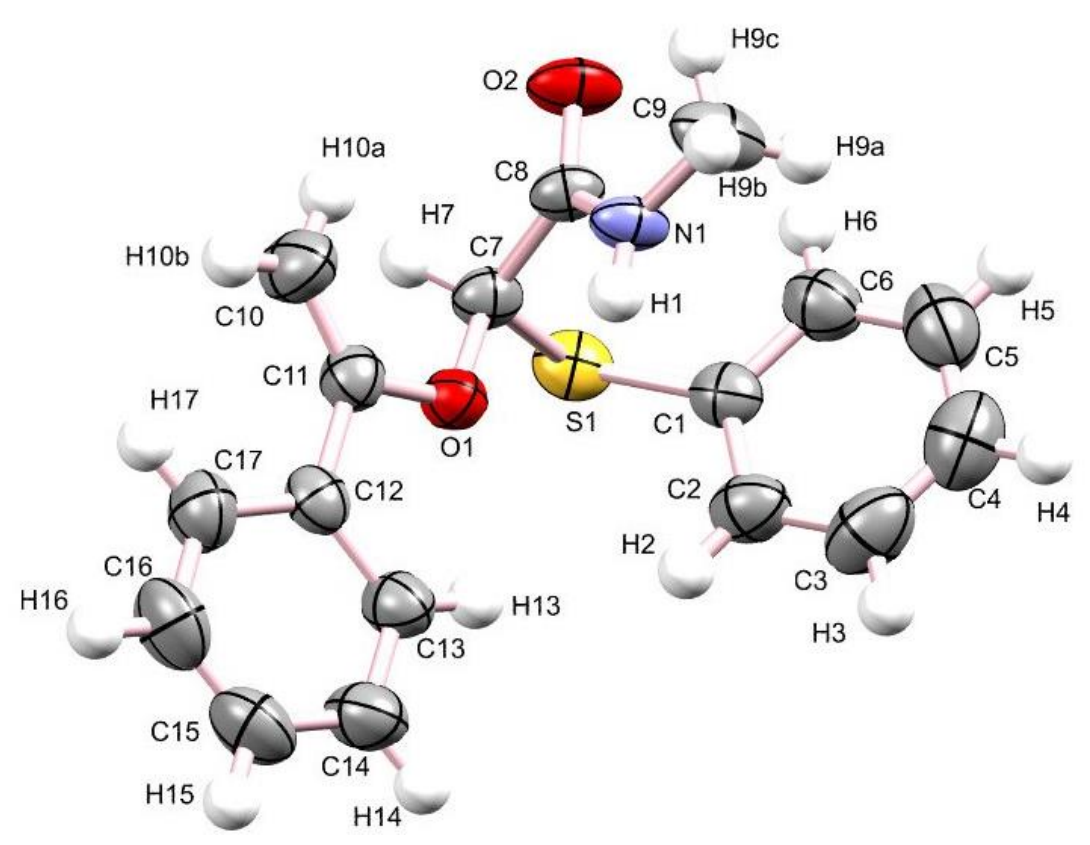

ORTEP Diagram of $\mathbf{1 1}$ (thermal ellipsoids are shown with 50\% probability). 


\section{Synthesis and Characterization of $\beta$-Keto Thioethers}

\section{Ethyl 2-((2-oxo-2-phenylethyl)thio)acetate (1a) ${ }^{4}$}<smiles>CCOC(=O)CSCC(=O)c1ccccc1</smiles>

flash column chromatography (Pet.ether/EtOAc =93/07) of the crude reaction mixture using silica gel afforded ethyl 2-((2-oxo-2-phenylethyl)thio)acetate 1a as pale yellow oil (1.0 g, $88 \%$ yield). $\boldsymbol{R} \mathbf{f}\left(\right.$ Pet. ether $/$ EtOAc = 95/05): 0.29; ${ }^{1} \mathbf{H}$ NMR (400 MHz, CDCl 3$) \delta$ 7.97-7.94 (m, 2H), 7.59-7.55 $(\mathrm{m}, 1 \mathrm{H}), 7.48-7.45(\mathrm{~m}, 2 \mathrm{H}), 4.17(\mathrm{q}, J=7.1 \mathrm{~Hz}, 2 \mathrm{H}), 4.02(\mathrm{~s}, 2 \mathrm{H}), 3.31(\mathrm{~s}, 2 \mathrm{H}), 1.25(\mathrm{t}, J=7.1 \mathrm{~Hz}$, 3H). ${ }^{13}$ C NMR (100 MHz, CDCl 3$) \delta 194.1,169.9,135.4,133.6,128.8,128.6,61.5,37.8,33.4$, 14.1. HRMS (ESI) m/z: [M+Na] ${ }^{+}$Calcd for $\mathrm{C}_{12} \mathrm{H}_{14} \mathrm{NaO}_{3} \mathrm{~S}$ 261.0556; Found: 261.0564. FTIR $\left(\mathbf{c m}^{-1}\right)$ 2983, 2365, 1731, 1676, 1598, 1275, 1155, 1024.

\section{Ethyl 2-((2-(4-methoxyphenyl)-2-oxoethyl)thio)acetate(1b)}<smiles>CCOC(=O)CSCC(=O)c1ccc(OC)cc1</smiles>

Following the general procedure, treatment of 2-((2-(4methoxyphenyl)-2-oxoethyl)thio)acetic acid(0.8 g, $3.329 \mathrm{mmol})$, EDC (1.273 g, $6.659 \mathrm{mmol})$, DMAP (0.202 g, $1.664 \mathrm{mmol})$ and $\operatorname{EtOH}(0.308 \mathrm{~g}, 0.4 \mathrm{~mL}, 6.659 \mathrm{mmol})$ in DCM $(53 \mathrm{~mL})$ at ambient temperature for $24 \mathrm{~h}$ followed by flash column chromatography $($ Pet.ether/EtOAc $=92 / 08)$ of the crude reaction mixture using silica gel afforded ethyl 2-((2-oxo-2-phenylethyl)thio)acetate $\mathbf{1 b}$ as pale yellow oil ( $0.660 \mathrm{~g}, 74 \%$ yield).

$\boldsymbol{R}_{\mathbf{f}}\left(\right.$ Pet. ether /EtOAc = 90/10): 0.21; ${ }^{1} \mathbf{H}$ NMR (400 MHz, CDCl3) $\delta$ 7.94-7.91 (m, 2H), 6.93-6.90 (m, 2H), $4.16(\mathrm{q}, J=7.1 \mathrm{~Hz}, 2 \mathrm{H}), 3.96(\mathrm{~s}, 2 \mathrm{H}), 3.85(\mathrm{~s}, 3 \mathrm{H}), 3.30(\mathrm{~s}, 2 \mathrm{H}), 1.25$ (t, $J=7.1 \mathrm{~Hz}, 3 \mathrm{H})$. ${ }^{13}$ C NMR (100 MHz, CDCl3) $\delta 192.8,170.0,163.9,131.0,128.4,114.0,61.6,55.6,37.6,33.5$, 14.2. HRMS (ESI) m/z: [M+Na] ${ }^{+}$Calcd for $\mathrm{C}_{13} \mathrm{H}_{16} \mathrm{NaO}_{4} \mathrm{~S} 291.0662$; Found: 291.0664 . FTIR (cm ${ }^{-}$ 1) 2936, 1733, 1667, 1599, 1511, 1260, 1175, 1025.

\section{Ethyl 2-((2-oxo-2-(p-tolyl)ethyl)thio)acetate (1c)}

Following the general procedure, treatment of 2-((2-oxo-2-(p-tolyl)ethyl)thio)acetic acid ( $1 \mathrm{~g}, 4.46$ mmol), EDC (1.705 g, $8.92 \mathrm{mmol})$, DMAP (0.272 g, $2.23 \mathrm{mmol})$ and EtOH (0.410 g, $0.52 \mathrm{~mL}$, 
$9.51 \mathrm{mmol})$ in DCM $(75 \mathrm{~mL})$ at ambient temperature for $24 \mathrm{~h}$ followed by flash column<smiles>CCOC(=O)CSCC(=O)c1ccc(C)cc1</smiles>
chromatography (Pet.ether/EtOAc $=95 / 05$ ) of the crude reaction mixture using silica gel afforded ethyl 2-((2-oxo-2(p-tolyl)ethyl)thio)acetate $1 \mathrm{c}$ as pale yellow oil $(0.930 \mathrm{~g}, 83 \%$ yield).

$\boldsymbol{R}_{\mathbf{f}}($ Pet. ether $/ \mathrm{EtOAc}=95 / 05): 0.40 ;{ }^{1} \mathbf{H}$ NMR $\left(\mathbf{4 0 0} \mathbf{~ M H z}, \mathbf{C D C l}_{3}\right) \delta 7.87(\mathrm{~d}, J=8.2 \mathrm{~Hz}, 2 \mathrm{H}), 7.27$ (d, $J=8.0 \mathrm{~Hz}, 2 \mathrm{H}), 4.19$ (q, $J=7.1 \mathrm{~Hz}, 2 \mathrm{H}), 4.01(\mathrm{~s}, 2 \mathrm{H}), 3.33(\mathrm{~s}, 2 \mathrm{H}), 2.42$ (s, 3H), 1.27 (t, $J=$ $7.1 \mathrm{~Hz}, 3 \mathrm{H}) .{ }^{13} \mathbf{C}$ NMR (100 MHz, $\left.\mathbf{C D C l}_{3}\right) \delta$ 193.9, 167.0, 144.6, 133.0, 129.5, 128.8, 61.6, 37.7, 33.5, 21.8, 14.2. HRMS (ESI) m/z: [M+Na] ${ }^{+}$Calcd for $\mathrm{C}_{13} \mathrm{H}_{16} \mathrm{NaO}_{3} \mathrm{~S}$ 275.0718; Found 275.0721. FTIR $\left(\mathbf{c m}^{-1}\right)$ 2982, 1735, 1605, 1278, 1219, 1028.

Ethyl 2-((2-(4-bromophenyl)-2-oxoethyl)thio)acetate (1d)<smiles>CCOC(=O)CSCC(=O)c1ccc(Br)cc1</smiles>

Following the general procedure, treatment of 2-((2-)(4bromophenyl)-2-oxoethyl)thio)acetic acid (0.750 g, $2.59 \mathrm{mmol})$,

EDC (0.994 g, $5.18 \mathrm{mmol})$, DMAP (0.158 g, $1.30 \mathrm{mmol})$ and EtOH $(0.239 \mathrm{~g}, 0.3 \mathrm{~mL}, 5.18 \mathrm{mmol})$ in DCM $(41 \mathrm{~mL})$ at ambient temperature for $24 \mathrm{~h}$ followed by flash column chromatography (Pet.ether/EtOAc $=95 / 05)$ of the crude reaction mixture using silica gel afforded ethyl 2-((2-(4-bromophenyl)-2-oxoethyl)thio)acetate 1d as light yellow oil (0.750 g, 91\% yield).

$\boldsymbol{R}_{\mathbf{f}}\left(\right.$ Pet. ether/EtOAc $=$ 90/10): 0.31; ${ }^{1} \mathbf{H}$ NMR (400 MHz, CDCl3) $\delta 7.82(\mathrm{~d}, J=8.5 \mathrm{~Hz}, 2 \mathrm{H}), 7.60$ $(\mathrm{d}, J=8.4 \mathrm{~Hz}, 2 \mathrm{H}), 4.17(\mathrm{q}, J=7.1 \mathrm{~Hz}, 2 \mathrm{H}), 3.97(\mathrm{~s}, 2 \mathrm{H}), 3.30(\mathrm{~s}, 2 \mathrm{H}), 1.26(\mathrm{t}, J=7.1 \mathrm{~Hz}, 3 \mathrm{H})$. ${ }^{13}$ C NMR (100 MHz, CDCl 3$) \delta 193.2,169.8,134.2,132.2,130.3,128.9,61.7,37.7,33.5,14.2$. HRMS (ESI) m/z: [M+Na] ${ }^{+}$Calcd for $\mathrm{C}_{12} \mathrm{H}_{13} \mathrm{BrNaO}_{3} \mathrm{~S}$ 338.9661; Found: 338.9662. FTIR (cm 1)2983, 2902, 1747, 1687, 1586, 1394, 1310, 1174, 1031.

\section{Ethyl 2-((2-(4-chlorophenyl)-2-oxoethyl)thio)acetate (1e)}<smiles>CCOC(=O)CSCC(=O)c1ccc(Cl)cc1</smiles>

Following the general procedure, treatment of 2-((2-)(4chlorophenyl)-2-oxoethyl)thio)acetic $\operatorname{acid}(0.250 \mathrm{~g}, 1.02 \mathrm{mmol})$, EDC (0.390 g, $2.04 \mathrm{mmol}), \mathrm{DMAP}(0.062 \mathrm{~g}, 0.51 \mathrm{mmol})$ and EtOH $(0.094 \mathrm{~g}, 0.1 \mathrm{~mL}, 2.04 \mathrm{mmol})$ in DCM $(17 \mathrm{~mL})$ at ambient temperature for $24 \mathrm{~h}$ followed by flash column chromatography (Pet.ether/EtOAc $=95 / 05)$ of the 
crude reaction mixture using silica gel afforded ethyl 2-((2-(4-chlorophenyl)-2oxoethyl)thio)acetate 1 e as pale yellow oil ( $0.251 \mathrm{~g}, 90 \%$ yield $)$.

$\boldsymbol{R}_{\mathbf{f}}\left(\right.$ Pet. ether/EtOAc = 90/10): 0.31; ${ }^{1} \mathbf{H}$ NMR (400 MHz, CDCl3) $\delta 7.90(\mathrm{~d}, J=8.6 \mathrm{~Hz}, 2 \mathrm{H}), 7.44$ $(\mathrm{d}, J=8.6 \mathrm{~Hz}, 2 \mathrm{H}), 4.17(\mathrm{q}, J=7.1 \mathrm{~Hz}, 2 \mathrm{H}), 3.98(\mathrm{~s}, 2 \mathrm{H}), 3.30(\mathrm{~s}, 2 \mathrm{H}), 1.26(\mathrm{t}, J=7.1 \mathrm{~Hz}, 3 \mathrm{H})$. ${ }^{13}$ C NMR (100 MHz, CDCl3) $\delta$ 193.0, 169.8, 140.2, 133.8, 130.2, 129.2, 61.7, 37.7, 33.5, 14.2. HRMS (ESI) m/z: [M+Na] ${ }^{+}$Calcd for $\mathrm{C}_{12} \mathrm{H}_{13} \mathrm{ClNaO}_{3} \mathrm{~S}$ 295.0166; Found 295.0172. FTIR (cm1) 2983, 1733, 1677, 1588, 1400, 1273, 1160, 1091, 1028.

Ethyl 2-((2-(4-fluorophenyl)-2-oxoethyl)thio)acetate (1f)<smiles>CCOC(=O)CSCC(=O)c1ccc(F)cc1</smiles>

Following the general procedure, treatment of 2-((2-)(4fluorophenyl)-2-oxoethyl)thio)acetic $\operatorname{acid}(0.450 \mathrm{~g}, 1.971 \mathrm{mmol})$, EDC (0.755 g, $3.942 \mathrm{mmol})$, DMAP (0.120 g, $0.985 \mathrm{mmol})$ and EtOH (0.181 g, $0.2 \mathrm{~mL}, 3.942 \mathrm{mmol})$ in DCM (41 mL) at ambient temperature for $24 \mathrm{~h}$ followed by flash column chromatography (Pet.ether/EtOAc $=95 / 05)$ of the crude reaction mixture using silica gel afforded ethyl 2-((2-(4-fluorophenyl)-2oxoethyl)thio)acetate $\mathbf{1 f}$ as pale yellow semi-solid ( $0.420 \mathrm{~g}, 83 \%$ yield).

$\boldsymbol{R}_{\mathbf{f}}\left(\right.$ Pet. ether $/$ EtOAc = 90/10): 0.32; ${ }^{1} \mathbf{H}$ NMR (400 MHz, CDCl3) $\delta$ 8.01-7.96 (m,2H), 7.15-7.10 $(\mathrm{m}, 2 \mathrm{H}), 4.16(\mathrm{q}, J=7.1 \mathrm{~Hz}, 2 \mathrm{H}), 3.98(\mathrm{~s}, 2 \mathrm{H}), 3.30(\mathrm{~s}, 2 \mathrm{H}), 1.25(\mathrm{t}, J=7.1 \mathrm{~Hz}, 3 \mathrm{H}) .{ }^{13} \mathbf{C}$ NMR (100 MHz, CDCl3) $\delta 192.6,169.8,166.1(\mathrm{~d}, J=256.5 \mathrm{~Hz}), 131.9(\mathrm{~d}, J=2.8 \mathrm{~Hz}), 131.4(\mathrm{~d}, J=$ $9.4 \mathrm{~Hz}), 116.0(\mathrm{~d}, J=21.9 \mathrm{~Hz}), 61.6,37.7,33.5,14.2$. HRMS (ESI) m/z: $[\mathrm{M}+\mathrm{Na}]^{+}$Calcd for $\mathrm{C}_{12} \mathrm{H}_{13} \mathrm{FNaO}_{3} \mathrm{~S}$ 279.0462; Found 279.0467. FTIR (cm $\left.{ }^{-1}\right)$ 2984, 2363, 1734, 1677, 1596, 1414, 1276, 1026.

\section{Ethyl 2-((2-(4-cyanophenyl)-2-oxoethyl)thio)acetate (1g)}<smiles>CCOC(=O)CSCC(=O)c1ccc(C#N)cc1</smiles>

Following the general procedure, treatment of 2-((2-)(4cyanophenyl)-2-oxoethyl)thio)acetic acid (1.1 g, $4.7 \mathrm{mmol})$, EDC (1.8 g, $9.51 \mathrm{mmol})$, DMAP (0.291 g, $2.38 \mathrm{mmol})$ and EtOH $(0.438$

$\mathrm{g}, 0.6 \mathrm{~mL}, 9.51 \mathrm{mmol})$ in DCM $(75 \mathrm{~mL})$ at ambient temperature for $24 \mathrm{~h}$ followed by flash column chromatography (Pet.ether/EtOAc $=85 / 15)$ of the crude reaction mixture using silica gel afforded ethyl 2-((2-(4-cyanophenyl)-2-oxoethyl)thio)acetate $1 \mathrm{~g}$ as white solid (0.9 g, $69 \%$ yield). 
$\boldsymbol{R}_{\mathbf{f}}($ Pet. ether $/$ EtOAc $=95 / 05): 0.15 ; \mathrm{mp} 53-55{ }^{\circ} \mathrm{C} ;{ }^{1} \mathbf{H}$ NMR $\left(400 \mathrm{MHz}, \mathbf{C D C l}_{3}\right) \delta 8.06(\mathrm{~d}, J=$ $8.6 \mathrm{~Hz}, 2 \mathrm{H}), 7.78(\mathrm{~d}, J=8.6 \mathrm{~Hz}, 2 \mathrm{H}), 4.18(\mathrm{q}, J=7.0 \mathrm{~Hz}, 2 \mathrm{H}), 4.01(\mathrm{~s}, 2 \mathrm{H}), 3.30(\mathrm{~s}, 2 \mathrm{H}), 1.27(\mathrm{t}$, $J=7.1 \mathrm{~Hz}, 3 \mathrm{H}) .{ }^{13} \mathbf{C}$ NMR (100 MHz, CDCl3) $\delta 192.7,169.7,138.5,132.7,129.2,117.9,116.9$, 61.8, 37.8, 33.5, 14.2. HRMS (ESI) m/z: $[\mathrm{M}+\mathrm{Na}]^{+}$Calcd for $\mathrm{C}_{13} \mathrm{H}_{13} \mathrm{NO}_{3} \mathrm{NaS}$ 286.0508; Found 286.0515. FTIR (cm-1) 2986, 2232, 1735, 1682, 1606, 1301.

\section{Ethyl 2-((2-(4-nitrophenyl)-2-oxoethyl)thio)acetate (1h)}<smiles>CCOC(=O)CSCC(=O)c1ccc([N+](=O)[O-])cc1</smiles>

Following the general procedure, treatment of 2-((2-(4nitrophenyl)-2-oxoethyl)thio)acetic acid (0.7 g, $2.7 \mathrm{mmol})$, EDC (1.1 g, $5.5 \mathrm{mmol})$, DMAP (0.168 g, $1.37 \mathrm{mmol})$ and EtOH $(0.25$ $\mathrm{g}, 0.32 \mathrm{~mL}, 5.5 \mathrm{mmol})$ in DCM $(43 \mathrm{~mL})$ at ambient temperature

for $24 \mathrm{~h}$ followed by flash column chromatography (Pet.ether/EtOAc $=85 / 15)$ of the crude reaction mixture using silica gel afforded Ethyl 2-((2-(4-nitrophenyl)-2-oxoethyl)thio)acetate $\mathbf{1 h}$ as yellow solid (0.8 g, $78 \%$ yield).

$\boldsymbol{R}_{\mathbf{f}}($ Pet. ether $/ \mathrm{EtOAc}=90 / 10): 0.18 ; \mathrm{mp} 38-40{ }^{\circ} \mathrm{C} ;{ }^{1} \mathbf{H}$ NMR (400 MHz, CDCl$) ~ \delta ~ 8.33-8.30(\mathrm{~m}$, $2 \mathrm{H}), 8.14-8.11(\mathrm{~m}, 2 \mathrm{H}), 4.18(\mathrm{q}, J=7.1 \mathrm{~Hz}, 2 \mathrm{H}), 4.04(\mathrm{~s}, 2 \mathrm{H}), 3.31(\mathrm{~s}, 2 \mathrm{H}), 1.27$ (t, $J=7.1 \mathrm{~Hz}$, 3H). ${ }^{13}$ C NMR (100 MHz, CDCl3) $\delta$ 192.5, 169.7, 150.6, 140.0, 129.9, 124.1, 61.8, 38.0, 33.5, 14.2. HRMS (ESI) m/z: [M+Na] ${ }^{+}$Calcd for $\mathrm{C}_{12} \mathrm{H}_{13} \mathrm{NNaO}_{5} \mathrm{~S}$ 306.0407; Found: 306.0414. FTIR $\left(\mathbf{c m}^{-1}\right)$ 2904, 1734, 1684, 1527, 1348, 1272, 1155, 1028.

\section{Ethyl 2-((2-(3-bromophenyl)-2-oxoethyl)thio)acetate (1i)}

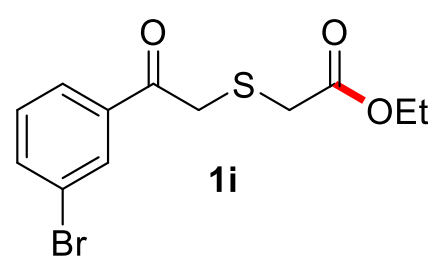

Following the general procedure, treatment of 2-((2-(3-bromophenyl)2-oxoethyl)thio)acetic acid (1.3 g, $4.7 \mathrm{mmol})$, EDC (1.8 g, 9.51 mmol), DMAP (0.291 g, $2.38 \mathrm{mmol})$ and $\mathrm{EtOH}(0.438 \mathrm{~g}, 0.6 \mathrm{~mL}, 9.51$ $\mathrm{mmol})$ in DCM $(75 \mathrm{~mL})$ at ambient temperature for $24 \mathrm{~h}$ followed by flash column chromatography $($ Pet.ether/EtOAc $=95 / 05)$ of the crude reaction mixture using silica gel afforded ethyl 2-((2-(3-bromophenyl)-2-oxoethyl)thio)acetate $\mathbf{1 i}$ as pale yellow oil (1.1 g, 76 $\%$ yield).

$\boldsymbol{R}_{\mathbf{f}}\left(\right.$ Pet. ether $/$ EtOAc = 95/05): 0.35; ${ }^{1} \mathbf{H}$ NMR (400 MHz, CDCl3) $\delta$ 8.09-8.08 (m, 1H), 7.88-7.86 (m, 1H), 7.71-7.69 (m, 1H), $7.35(\mathrm{t}, J=7.9 \mathrm{~Hz}, 1 \mathrm{H}), 4.18(\mathrm{q}, J=7.0 \mathrm{~Hz}, 2 \mathrm{H}), 3.98(\mathrm{~s}, 2 \mathrm{H}), 3.30$ $(\mathrm{s}, 2 \mathrm{H}), 1.26$ (t, $J=7.1 \mathrm{~Hz}, 3 \mathrm{H}) .{ }^{13} \mathbf{C}$ NMR (100 MHz, CDCl 3$) \delta 192.8,169.8,137.2,136.5,131.8$, 
130.4 127.3, 123.2, 61.7, 37.7, 33.5, 14.2. HRMS (ESI) m/z: $[\mathrm{M}+\mathrm{Na}]^{+}$Calcd for $\mathrm{C}_{12} \mathrm{H}_{13} \mathrm{BrO}_{3} \mathrm{NaS}$ 338.9661; Found 338.9663. FTIR ( cm$\left.^{-1}\right)$ 3065, 2983, 2903, 1740, 1565, 1301.

\section{Ethyl 2-((2-(3-chlorophenyl)-2-oxoethyl)thio)acetate (1j)}<smiles>CCOC(=O)CSCC(=O)c1cccc(Cl)c1</smiles>

Following the general procedure, treatment of 2-((2-(3chlorophenyl)-2-oxoethyl)thio)acetic acid (1.1 g, $4.7 \mathrm{mmol})$, EDC (1.8 g, $9.51 \mathrm{mmol})$, DMAP (0.291 g, $2.38 \mathrm{mmol})$ and EtOH (0.438 $\mathrm{g}, 0.6 \mathrm{~mL}, 9.51 \mathrm{mmol})$ in DCM $(75 \mathrm{~mL})$ at ambient temperature for $24 \mathrm{~h}$ followed by flash column chromatography (Pet.ether/EtOAc $=$ 95/05) of the crude reaction mixture using silica gel afforded ethyl 2-((2-(3-chlorophenyl)-2oxoethyl)thio)acetate $\mathbf{1} \mathbf{j}$ as pale yellow oil ( $1.1 \mathrm{~g}, 90 \%$ yield).

$\boldsymbol{R}_{\mathbf{f}}($ Pet. ether $/ \mathrm{EtOAc}=95 / 05): 0.35 ;{ }^{1} \mathbf{H}$ NMR (400 MHz, $\left.\mathbf{C D C l}_{3}\right) \delta$ 7.93-7.92 (m, 1H), 7.83-7.81 (m, 1H), 7.55-7.53 (m, 1H), 7.41 (t, $J=7.9 \mathrm{~Hz}, 1 \mathrm{H}), 4.17$ (q, $J=7.1 \mathrm{~Hz}, 2 \mathrm{H}), 3.98$ (s, 2H), 3.30 (s, 2H), 1.26 (t, $J=7.2 \mathrm{~Hz}, 3 \mathrm{H}) .{ }^{13} \mathbf{C}$ NMR (100 MHz, $\left.\mathbf{C D C l}_{3}\right) \delta 192.8,169.8,137.0,135.2,133.5$, 130.2, 128.8, 126.8, 61.7, 37.8, 33.5, 14.2. HRMS (ESI) m/z: $[\mathrm{M}+\mathrm{Na}]^{+} \mathrm{Calcd}$ for $\mathrm{C}_{12} \mathrm{H}_{13} \mathrm{ClO}_{3} \mathrm{NaS}$ 295.0166; Found 295.0172. FTIR ( cm$\left.^{-1}\right)$ 3465, 2984, 2935, 1742, 1569, 1241.

Ethyl 2-((2-(3-nitrophenyl)-2-oxoethyl)thio)acetate (1k)<smiles>CCOC(=O)CSCC(=O)c1cccc([N+](=O)[O-])c1</smiles>

Following the general procedure, treatment of 2-((2-(3-nitrophenyl)-2oxoethyl)thio)acetic acid $(0.5 \mathrm{~g}, 1.96 \mathrm{mmol})$, EDC (0.751 g, 3.91 mmol), DMAP (0.120 g, $0.98 \mathrm{mmol})$ and EtOH $(0.180 \mathrm{~g}, 0.23 \mathrm{~mL}, 3.91$ $\mathrm{mmol})$ in DCM $(31 \mathrm{~mL})$ at ambient temperature for $24 \mathrm{~h}$ followed by flash column chromatography (Pet.ether/EtOAc $=85 / 15)$ of the crude reaction mixture using silica gel afforded ethyl 2-((2-(3-nitrophenyl)-2-oxoethyl)thio)acetate $\mathbf{1 k}$ as yellow solid (0.410 g, 74\% yield).

$\boldsymbol{R} \mathbf{f}($ Pet. ether $/$ EtOAc $=90 / 10): 0.18 ; \mathrm{mp} 53-55{ }^{\circ} \mathrm{C} ;{ }^{1} \mathbf{H}$ NMR $(400 \mathrm{MHz}, \mathbf{C D C l})$ ) $\delta$ 8.78-8.77 (m, $1 \mathrm{H}), 8.43-8.41(\mathrm{~m}, 1 \mathrm{H}), 8.30-8.28(\mathrm{~m}, 1 \mathrm{H}), 7.69$ (t, $J=8.0 \mathrm{~Hz}, 1 \mathrm{H}), 4.18$ (q, $J=7.1 \mathrm{~Hz}, 2 \mathrm{H}), 4.05$ (s, 2H), 3.31 (s, 2H), 1.26 (t, $J=7.1 \mathrm{~Hz}, 3 \mathrm{H}) .{ }^{13} \mathbf{C} \mathbf{~ N M R}\left(\mathbf{1 0 0} \mathbf{~ M H z}, \mathbf{C D C l}_{3}\right) \delta$ 191.9, 169.6, 148.6, 136.7, 134.3, 130.2, 127.8, 123.6, 61.8, 37.7, 33.5, 14.2. HRMS (ESI) m/z: $[\mathrm{M}+\mathrm{Na}]^{+}$Calcd for $\mathrm{C}_{12} \mathrm{H}_{13} \mathrm{NNaO}_{5} \mathrm{~S}$ 306.0407; Found 306.0410. FTIR ( $\left.\mathbf{c m}^{-1}\right)$ 2984, 1731, 1684, 1612, 1532, 1350, 1264, 1027. 


\section{Ethyl 2-((2-(2-methoxyphenyl)-2-oxoethyl)thio)acetate (11)}<smiles>CCOC(=O)CSCC(=O)c1ccccc1OC</smiles>

Following the general procedure, treatment of 2-((2-)(2methoxyphenyl)-2-oxoethyl)thio)acetic acid (0.6 g, $2.50 \mathrm{mmol})$, EDC (0.957 g, $4.99 \mathrm{mmol})$, DMAP (0.152 g, $1.25 \mathrm{mmol})$ and EtOH (0.230 $\mathrm{g}, 0.29 \mathrm{~mL}, 4.99 \mathrm{mmol})$ in DCM (39 mL) at ambient temperature for $24 \mathrm{~h}$ followed by flash column chromatography (Pet.ether/EtOAc $=90 / 10)$ of the crude reaction mixture using silica gel afforded ethyl 2-((2-(2-methoxyphenyl)-2-oxoethyl)thio)acetate 11 as white solid (0.513 g, 77\% yield). $\boldsymbol{R}_{\mathbf{f}}\left(\right.$ Pet. ether $/$ EtOAc = 90/10): 0.20; $\mathrm{mp} 50-52{ }^{\circ} \mathrm{C} ;{ }^{1} \mathbf{H}$ NMR $(400 \mathbf{~ M H z}, \mathbf{C D C l} 3) \delta 7.81\left(\mathrm{dd}, J_{l}=\right.$ $\left.7.7 \mathrm{HZ}, J_{2}=1.7 \mathrm{~Hz}, 1 \mathrm{H}\right), 7.50-7.46(\mathrm{~m}, 1 \mathrm{H}), 7.03-6.99(\mathrm{~m}, 1 \mathrm{H}), 6.96(\mathrm{~d}, J=8.4 \mathrm{~Hz}, 1 \mathrm{H}), 4.16(\mathrm{q}$, $J=7.1 \mathrm{~Hz}, 2 \mathrm{H}), 4.03(\mathrm{~s}, 2 \mathrm{H}), 3.90(\mathrm{~s}, 3 \mathrm{H}), 3.27(\mathrm{~s}, 2 \mathrm{H}), 1.25(\mathrm{t}, J=7.1 \mathrm{~Hz}, 3 \mathrm{H}) .{ }^{13} \mathrm{C}$ NMR (100 MHz, CDCl $) \delta 195.5,170.2,158.9,134.4,131.4,126.1,121.0,111.7,61.5,55.7,42.9,33.5,14.2$. HRMS (ESI) m/z: [M+Na] ${ }^{+}$Calcd for $\mathrm{C}_{13} \mathrm{H}_{16} \mathrm{NaO}_{4} \mathrm{~S}$ 291.0662; Found 291.0667. FTIR (cm 1) 2981, 1732, 1665, 1596, 1484, 1301, 1162, 1024.

Ethyl 2-((2-(2-chlorophenyl)-2-oxoethyl)thio)acetate (1m)<smiles>CCOC(=O)CSCC(=O)c1ccccc1Cl</smiles>

Following the general procedure, treatment of 2-((2-)(2chlorophenyl)-2-oxoethyl)thio)acetic acid (1.1 g, $4.7 \mathrm{mmol})$, EDC (1.8 g, $9.51 \mathrm{mmol})$, DMAP (0.291 g, $2.38 \mathrm{mmol})$ and EtOH (0.438 $\mathrm{g}, 0.6 \mathrm{~mL}, 9.51 \mathrm{mmol})$ in DCM $(75 \mathrm{~mL})$ at ambient temperature for $24 \mathrm{~h}$ followed by flash column chromatography $($ Pet.ether/EtOAc $=$ 95/05) of the crude reaction mixture using silica gel afforded ethyl 2-((2-(2-chlorophenyl)-2oxoethyl)thio)acetate $\mathbf{1 m}$ as pale yellow oil ( $0.9 \mathrm{~g}, 69 \%$ yield).

$\boldsymbol{R} \mathbf{f}\left(\right.$ Pet. ether $/$ EtOAc = 95/05): 0.35; ${ }^{1} \mathbf{H}$ NMR (400 MHz, CDCl3) $\delta$ 7.57-7.55 (m, 1H), 7.43-7.38 (m, 2H), 7.37-7.32 (m, 1H), 4.23-4.16 (m, 2H), $4.02(\mathrm{~s}, 2 \mathrm{H}), 3.30(\mathrm{~s}, 2 \mathrm{H}), 1.27(\mathrm{t}, J=7.1 \mathrm{~Hz}, 3 \mathrm{H})$. ${ }^{13}$ C NMR (100 MHz, CDCl3) $\delta 196.9,169.8,137.7,132.4,131.4,130.7,130.0,127.1,61.7,41.3$, 33.4, 14.3. HRMS (ESI) m/z: [M+Na] $]^{+}$Calcd for $\mathrm{C}_{12} \mathrm{H}_{13} \mathrm{ClO}_{3} \mathrm{NaS}$ 295.0166; Found 295.0172. FTIR $\left(\mathbf{c m}^{-1}\right)$ 3068, 2984, 2934, 1742, 1676, 1569.

\section{Ethyl 2-((2-(2-nitrophenyl)-2-oxoethyl)thio)acetate (1n)}

Following the general procedure, treatment of 2-((2-(2-nitrophenyl)-2-oxoethyl)thio)acetic acid (0.580 g, $2.27 \mathrm{mmol})$, EDC (0.870 g, $4.54 \mathrm{mmol})$, DMAP (0.139 g, $1.14 \mathrm{mmol})$ and EtOH (0.209 


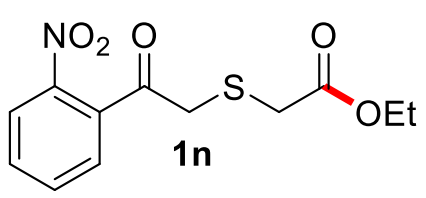

$\mathrm{g}, 0.27 \mathrm{~mL}, 4.54 \mathrm{mmol})$ in DCM $(36 \mathrm{~mL})$ at ambient temperature for $24 \mathrm{~h}$ followed by flash column chromatography (Pet.ether/EtOAc $=$ $85 / 15)$ of the crude reaction mixture using silica gel afforded ethyl 2((2-(2-nitrophenyl)-2-oxoethyl)thio)acetate $\mathbf{1 n}$ as yellow oil ( $0.510 \mathrm{~g}$,

$79 \%$ yield).

$\boldsymbol{R}_{\mathbf{f}}\left(\right.$ Pet. ether $/$ EtOAc $=$ 90/10): 0.19; ${ }^{1} \mathbf{H}$ NMR $\left(\mathbf{4 0 0} \mathbf{~ M H z}, \mathbf{C D C l}_{3}\right) \delta$ 8.03-8.01 (m, 1H), 7.69-7.65 (m, 1H), 7.57-7.53 (m, 1H), 7.49-7.47 (m, 1H), 4.07 (q, J = 7.1 Hz, 2H), $3.75(\mathrm{~s}, 2 \mathrm{H}), 3.24(\mathrm{~s}, 2 \mathrm{H})$, $1.16(\mathrm{t}, J=7.1 \mathrm{~Hz}, 3 \mathrm{H}) .{ }^{\mathbf{1 3}} \mathbf{C} \mathbf{~ N M R}\left(\mathbf{1 0 0} \mathbf{M H z}, \mathbf{C D C l}_{3}\right) \delta 196.1,169.4,145.4,135.8,134.3,130.9$, 128.7, 124.2, 61.33, 41.0, 33.2, 13.9. HRMS (ESI) m/z: $[\mathrm{M}+\mathrm{Na}]^{+}$Calcd for $\mathrm{C}_{12} \mathrm{H}_{13} \mathrm{NNaO}_{5} \mathrm{~S}$ 306.0407; Found 306.0410. FTIR (cm-1) 2920, 1734, 1696, 1529, 1347, 1197, 1021.

Ethyl 2-((2-(3,4-dimethoxyphenyl)-2-oxoethyl)thio)acetate (10)<smiles>CCOC(=O)CSCC(=O)c1ccc(OC)c(OC)c1</smiles>

Following the general procedure, treatment of 2-((2-(3,4dimethoxyphenyl)-2-oxoethyl)thio)acetic $\operatorname{acid}(0.7 \mathrm{~g}, 2.59 \mathrm{mmol})$, $\operatorname{EDC}(0.993 \mathrm{~g}, 5.18 \mathrm{mmol})$, DMAP $(0.158 \mathrm{~g}, 1.30 \mathrm{mmol})$ and EtOH $(0.239 \mathrm{~g}, 0.3 \mathrm{~mL}, 5.18 \mathrm{mmol})$ in DCM $(41 \mathrm{~mL})$ at ambient temperature for $24 \mathrm{~h}$ followed by flash column chromatography (Pet.ether/EtOAc $=85 / 15$ ) of the crude reaction mixture using silica gel afforded ethyl 2-((2-(3,4-dimethoxyphenyl)-2oxoethyl)thio)acetate 10 as white solid $(0.650 \mathrm{~g}, 85 \%$ yield $)$.

$\boldsymbol{R}_{\mathbf{f}}(\mathrm{Pet}$. ether /EtOAc $=90 / 10): 0.29 ; \mathrm{mp} 48-50{ }^{\circ} \mathrm{C} ;{ }^{1} \mathbf{H}$ NMR (400 MHz, $\left.\mathbf{C D C l}_{3}\right) \delta$ 7.59-7.56 (m, 1H), 7.53-7.52 (m, 1H), 6.89-6.87 (m, 1H), $4.18(\mathrm{q}, J=7.1 \mathrm{~Hz}, 2 \mathrm{H}), 3.98(\mathrm{~s}, 2 \mathrm{H}), 3.94(\mathrm{~s}, 3 \mathrm{H})$, 3.92 (s, 3H), 3.32 (s, 2H), 1.26 (t, $J=7.1 \mathrm{~Hz}, 3 \mathrm{H}) .{ }^{\mathbf{1 3}} \mathbf{C} \mathbf{~ N M R}\left(\mathbf{1 0 0} \mathbf{~ M H z}, \mathbf{C D C l}_{3}\right)$ 8193.0, 170.0, 153.9, 149.3, 128.7, 123.6, 110.8, 110.2, 61.6, 56.2, 56.1, 37.3, 33.6, 14.2. HRMS (ESI) m/z: $[\mathrm{M}+\mathrm{Na}]^{+}$Calcd for $\mathrm{C}_{14} \mathrm{H}_{18} \mathrm{NaO}_{5} \mathrm{~S}$ 321.0767; Found 321.0773. FTIR (cm $^{-}$ 1) $2978,2936,1719,1660,1590,1514,1416,1263,1024$.

Ethyl 2-((2-(3-bromo-4-fluorophenyl)-2-oxoethyl)thio)acetate (1p)<smiles>CCOC(=O)CSCC(=O)c1ccc(F)c(Br)c1</smiles>

Following the general procedure, treatment of 2-((2-(3-bromo-4fluorophenyl)-2-oxoethyl)thio)acetic $\operatorname{acid}(0.320 \mathrm{~g}, 1.04 \mathrm{mmol})$, EDC (0.400 g, $2.08 \mathrm{mmol})$, DMAP (0.064 g, $0.52 \mathrm{mmol})$ and EtOH $(0.096 \mathrm{~g}, 0.12 \mathrm{~mL}, 2.08 \mathrm{mmol})$ in DCM $(16 \mathrm{~mL})$ at ambient 
temperature for $24 \mathrm{~h}$ followed by flash column chromatography (Pet.ether/EtOAc $=95 / 05)$ of the crude reaction mixture using silica gel afforded ethyl 2-((2-(3-bromo-4-fluorophenyl)-2oxoethyl)thio)acetate $1 p$ as light yellow oil $(0.275 \mathrm{~g}, 79 \%$ yield).

$\boldsymbol{R}_{\mathbf{f}}($ Pet. ether /EtOAc $=90 / 10): 0.35 ;{ }^{1} \mathbf{H}$ NMR $\left(\mathbf{4 0 0} \mathbf{~ M H z}, \mathbf{C D C l}_{3}\right) \delta 8.19\left(\mathrm{dd}, J_{1}=6.6 \mathrm{~Hz}, J_{2}=2.1\right.$ $\mathrm{Hz}, 1 \mathrm{H}), 7.93-7.89(\mathrm{~m}, 1 \mathrm{H}), 7.22-7.18(\mathrm{~m}, 1 \mathrm{H}), 4.18(\mathrm{q}, J=7.1 \mathrm{~Hz}, 2 \mathrm{H}), 3.96(\mathrm{~s}, 2 \mathrm{H}), 3.30(\mathrm{~s}, 2 \mathrm{H})$, 1.27 (t, $J=7.1 \mathrm{~Hz}, 3 \mathrm{H}) .{ }^{13} \mathbf{C}$ NMR (100 MHz, CDCl$) \delta 191.5,169.7,162.4(\mathrm{~d}, J=256.7 \mathrm{~Hz}), 134.7$ (d, $J=1.8 \mathrm{~Hz}), 133.0$ (d, $J=3.6 \mathrm{~Hz}), 130.0(\mathrm{~d}, J=8.6 \mathrm{~Hz}), 116.9$ (d, $J=23.0 \mathrm{~Hz}), 110.1$ (d, $J=$ $21.8 \mathrm{~Hz}$ ), 61.7, 37.6, 33.5, 14.2. HRMS (ESI) m/z: $[\mathrm{M}+\mathrm{Na}]^{+}$Calcd for $\mathrm{C}_{12} \mathrm{H}_{12} \mathrm{BrFNaO}_{3} \mathrm{~S}$ 356.9567; Found 356.9552. FTIR ( cm$\left.^{-1}\right)$ 2983, 1734, 1679, 1588, 1492, 1391, 1265, 1027.

\section{Ethyl 2-((2-(naphthalen-2-yl)-2-oxoethyl)thio)acetate (1q)}<smiles>CCOC(=O)CSCC(=O)c1ccc2ccccc2c1</smiles>

Following the general procedure, treatment of 2-((2-(naphthalen2-yl)-2-oxoethyl)thio)acetic acid (0.5 g, $1.92 \mathrm{mmol})$, EDC (0.736 g, $3.84 \mathrm{mmol})$, DMAP $(0.117 \mathrm{~g}, 0.96 \mathrm{mmol})$ and EtOH $(0.177 \mathrm{~g}$, $0.22 \mathrm{~mL}, 3.84 \mathrm{mmol})$ in DCM (30 $\mathrm{mL})$ at ambient temperature for $24 \mathrm{~h}$ followed by flash column chromatography (Pet.ether/EtOAc $=95 / 05)$ of the crude reaction mixture using silica gel afforded ethyl 2-((2-(naphthalen-2-yl)-2-oxoethyl)thio)acetate 1q as pale yellow oil ( $0.4 \mathrm{~g}, 74 \%$ yield $)$.

$\boldsymbol{R}_{\mathbf{f}}($ Pet. ether /EtOAc $=90 / 10): 0.29 ;{ }^{1} \mathbf{H}$ NMR $\left(\mathbf{4 0 0} \mathbf{~ M H z}, \mathbf{C D C l}_{3}\right) \delta 8.49(\mathrm{~s}, 1 \mathrm{H}), 8.03-8.00(\mathrm{~m}$, $1 \mathrm{H})$, 7.97-7.95 (m, 1H), 7.91-7.86 (m, 2H), 7.63-7.54 (m, 2H), 4.22-4.16 (m,4H), $3.36(\mathrm{~s}, 2 \mathrm{H})$, $1.27(\mathrm{t}, J=7.1 \mathrm{~Hz}, 3 \mathrm{H}) .{ }^{13} \mathbf{C}$ NMR (100 MHz, $\left.\mathbf{C D C l}_{3}\right) \delta 194.2,170.0,135.9,132.8,132.6,130.6$, 129.8, 128.9, 128.8, 127.9, 127.0, 124.2,61.6, 37.9, 33.6, 14.2. HRMS (ESI) m/z: $[\mathrm{M}+\mathrm{Na}]^{+} \mathrm{Calcd}$ for $\mathrm{C}_{16} \mathrm{H}_{16} \mathrm{NaO}_{3} \mathrm{~S}$ 311.0712; Found: 311.0718. FTIR ( $\left.\mathbf{c m}^{-1}\right)$ 2982, 1732, 1673, 1626, 1468, 1293 , 1027.

\section{Ethyl 2-((2-(naphthalen-1-yl)-2-oxoethyl)thio)acetate(1r)}

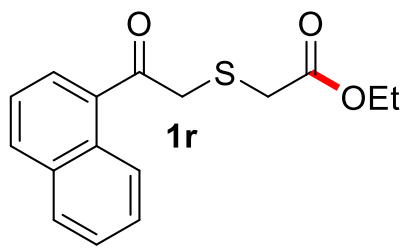

Following the general procedure, treatment of 2-((2-(naphthalen-1-yl)2-oxoethyl)thio)acetic acid (0.6 g, $2.31 \mathrm{mmol})$, EDC (0.883 g, 4.61 mmol), DMAP (0.141 g, $1.52 \mathrm{mmol})$ and EtOH $(0.212 \mathrm{~g}, 0.27 \mathrm{~mL}, 4.61$ $\mathrm{mmol})$ in DCM $(53 \mathrm{~mL})$ at ambient temperature for $24 \mathrm{~h}$ followed by flash column chromatography (Pet.ether/EtOAc $=95 / 05)$ of the crude reaction mixture using silica 
gel afforded ethyl 2-((2-(naphthalen-1-yl)-2-oxoethyl)thio)acetateas 1r pale yellow solid (0.665 g, $75 \%$ yield).

$\boldsymbol{R}_{\mathbf{f}}($ Pet. ether $/ \mathrm{EtOAc}=90 / 10): 0.29 ; \mathrm{mp} \mathrm{38-40}{ }^{\circ} \mathrm{C} ;{ }^{1} \mathbf{H}$ NMR (400 MHz, CDCl 3$) \delta 8.62(\mathrm{~d}, J=$ $8.6 \mathrm{~Hz}, 1 \mathrm{H}), 8.00(\mathrm{~d}, J=8.2 \mathrm{~Hz}, 1 \mathrm{H}), 7.93-7.91(\mathrm{~m}, 1 \mathrm{H}), 7.87(\mathrm{~d}, J=8.1 \mathrm{~Hz}, 1 \mathrm{H}), 7.63-7.58$ (m, 1H), 7.56-7.47 (m, 2H), 4.19 (q, $J=7.1 \mathrm{~Hz}, 2 \mathrm{H}), 4.14(\mathrm{~s}, 2 \mathrm{H}), 3.37$ (s, 2H), 1.26 (t, $J=7.1 \mathrm{~Hz}$, 3H), ${ }^{13}$ C NMR (100 MHz, CDCl 3$) \delta 197.8,170.0,134.2,134.1,133.4,130.7,128.6,128.4,128.3$, 126.7, 125.8, 124.4, 61.6, 40.8, 33.5, 14.2. HRMS (ESI) m/z: $[\mathrm{M}+\mathrm{Na}]{ }^{+}$Calcd for $\mathrm{C}_{16} \mathrm{H}_{16} \mathrm{NaO}_{3} \mathrm{~S}$ 311.0712; Found: 311.0717. FTIR (cm-1) 2982, 1732, 1675, 1285, 1170, 1027.

\section{Ethyl 2-((2-(3,5-difluorophenyl)-2-oxoethyl)thio)acetate (1s)}<smiles>CCOC(=O)CSCC(=O)c1cc(F)cc(F)c1</smiles>

Following the general procedure, treatment of $2-((2-(3,5-$ difluorophenyl)-2-oxoethyl)thio)acetic acid (0.8 g, $3.24 \mathrm{mmol})$, EDC (1.2 g, $6.49 \mathrm{mmol})$, DMAP (0.198 g, $1.62 \mathrm{mmol})$ and EeOH (0.299 $\mathrm{g}, 0.4 \mathrm{~mL}, 6.49 \mathrm{mmol})$ in DCM $(51 \mathrm{~mL})$ at ambient temperature for $24 \mathrm{~h}$ followed by flash column chromatography (Pet.ether/EtOAc $=95 / 05)$ of the crude reaction mixture using silica gel afforded ethyl 2-((2-(3,5-difluorophenyl)-2oxoethyl)thio)acetate $1 \mathrm{~s}$ as pale yellow oil $(0.810 \mathrm{~g}, 91 \%$ yield $)$.

$\boldsymbol{R}_{\mathbf{f}}\left(\right.$ Pet. ether /EtOAc = 90/10): 0.49; ${ }^{1} \mathbf{H}$ NMR (400 MHz, $\left.\mathbf{C D C l}_{3}\right) \delta$ 7.47- 7.42 (m, 2H), 7.04$6.98(\mathrm{~m}, 1 \mathrm{H}), 4.15(\mathrm{q}, J=7.1 \mathrm{~Hz}, 2 \mathrm{H}), 3.94(\mathrm{~s}, 2 \mathrm{H}), 3.28(\mathrm{~s}, 2 \mathrm{H}), 1.24(\mathrm{t}, J=7.2 \mathrm{~Hz}, 3 \mathrm{H}) .{ }^{13} \mathbf{C ~ N M R}$ (100 MHz, CDCl3) $\delta 191.6(\mathrm{t}, J=2.4 \mathrm{~Hz}), 169.6,163.2(\mathrm{~d}, J=251.3 \mathrm{~Hz}), 163.0(\mathrm{~d}, J=251.2 \mathrm{~Hz})$, $138.3(\mathrm{t}, J=7.7 \mathrm{~Hz}), 111.8(\mathrm{~d}, J=26.3 \mathrm{~Hz}), 111.6(\mathrm{~d}, J=11.3 \mathrm{~Hz}), 108.8$ (t, $J=25.4 \mathrm{~Hz}), 61.7$, 37.6, 33.4, 14.1. HRMS (ESI) m/z: $[\mathrm{M}+\mathrm{Na}]^{+}$Calcd for $\mathrm{C}_{12} \mathrm{H}_{12} \mathrm{~F}_{2} \mathrm{NaO}_{3} \mathrm{~S}$ 297.0367; Found 297.0370. FTIR $\left(\mathbf{c m}^{-1}\right)$ 3088, 2986, 1742, 1682, 1594, 1442, 1329, 1120, 987.

\section{Ethyl 2-((2-oxo-2-(thiophen-2-yl)ethyl)thio)acetate (1t)}<smiles>CCOC(=O)CSCC(=O)c1cccs1</smiles>

$1 t$

Following the general procedure, treatment of 2-((2-(furan-2-yl)-2oxoethyl)thio)acetic $\operatorname{acid}(0.6 \mathrm{~g}, 2.77 \mathrm{mmol})$, EDC (1.063 g, 5.54 mmol), DMAP (0.169 g, $1.385 \mathrm{mmol})$ and EtOH (0.255 g, $0.3 \mathrm{~mL}, 9.51$ $\mathrm{mmol})$ in DCM (44 $\mathrm{mL})$ at ambient temperature for $24 \mathrm{~h}$ followed by flash column chromatography $($ Pet.ether/EtOAc $=95 / 05)$ of the crude reaction mixture using silica 
gel afforded Ethyl 2-((2-oxo-2-(thiophen-2-yl)ethyl)thio)acetate 1t as pale yellow oil (0.495 g, $73 \%$ yield).

$\boldsymbol{R}_{\mathbf{f}}($ Pet. ether $/$ EtOAc $=90 / 10): 0.31 ;{ }^{1} \mathbf{H}$ NMR (400 MHz, CDCl3) $\delta 7.75\left(\mathrm{dd}, J_{1}=3.8 \mathrm{~Hz}, J_{2}=\right.$ $1.0 \mathrm{~Hz}, 1 \mathrm{H}), 7.67\left(\mathrm{dd}, J_{1}=4.9 \mathrm{~Hz}, J_{2}=1.0 \mathrm{~Hz}, 1 \mathrm{H}\right), 7.13\left(\mathrm{dd}, J_{1}=4.9 \mathrm{~Hz}, J_{2}=3.9 \mathrm{~Hz}, 1 \mathrm{H}\right), 4.17$ (q, $J=7.1 \mathrm{~Hz}, 2 \mathrm{H}), 3.91(\mathrm{~s}, 2 \mathrm{H}), 3.34(\mathrm{~s}, 2 \mathrm{H}), 1.25(\mathrm{t}, J=7.1 \mathrm{~Hz}, 3 \mathrm{H}) .{ }^{13} \mathrm{C}$ NMR (100 MHz, $\left.\mathbf{C D C l}_{3}\right) \delta 187.4,169.9,142.6,134.6,133.0,128.4,61.6,38.1,33.6,14.2$. HRMS (ESI) m/z: $[\mathrm{M}+\mathrm{Na}]^{+}$Calcd for $\mathrm{C}_{10} \mathrm{H}_{12} \mathrm{NaO}_{3} \mathrm{~S}_{2}$ 267.0120; Found 267.0125. FTIR (cm1) $3095,2983,1731,1653,1515,1413,1291,1156,1027$.

\section{Methyl 2-((2-oxo-2-phenylethyl)thio)acetate $(1 \mathrm{u})^{8}$}<smiles>COC(=O)CSCC(=O)c1ccccc1</smiles>

Following the general procedure, treatment of 2-((2-oxo-2phenylethyl)thio)acetic acid(0.5 g, $2.387 \mathrm{mmol})$, EDC (0.9 g, 4.75 mmol), DMAP (0.145 g, $1.189 \mathrm{mmol})$ and $\mathrm{MeOH}(0.152 \mathrm{~g}, 0.2 \mathrm{~mL}$, $4.756 \mathrm{mmol})$ in DCM (38 $\mathrm{mL})$ at ambient temperature for $24 \mathrm{~h}$ followed by flash column chromatography (Pet.ether/EtOAc $=95 / 05)$ of the crude reaction mixture using silica gel afforded methyl 2-((2-oxo-2-phenylethyl)thio)acetateas 1u as pale yellow oil (0.475 g, 89\% yield).

$\boldsymbol{R}_{\mathbf{f}}\left(\right.$ Pet. ether $/$ EtOAc = 90/10): 0.49; ${ }^{1} \mathbf{H}$ NMR (400 MHz, CDCl3) $\delta$ 7.96- 7.94 (m, 2H), 7.597.55 (m, 1H), 7.48-7.44 (m, 2H), 4.01 (s, 2H), 3.71 (s, 3H), 3.33 (s, 2H). ${ }^{13}$ C NMR (100 MHz, $\left.\mathbf{C D C l}_{3}\right) \delta 194.1,170.4,135.4,133.7,128.8,128.7,52.6,37.8,33.2$. HRMS (ESI) m/z: [M+Na $]^{+}$ Calcd for $\mathrm{C}_{11} \mathrm{H}_{12} \mathrm{NaO}_{3} \mathrm{~S}$ 247.0399; Found 247.0405. FTIR (cm-1) 2952, 1738, 1675, 1277, 1159 , 1009.

\section{Butyl 2-((2-oxo-2-phenylethyl)thio)acetate (1v)}<smiles>CCCCOC(=O)CSCC(=O)c1ccccc1</smiles>

Following the general procedure, treatment of 2-((2-oxo-2phenylethyl)thio)acetic acid (0.5 g, $2.38 \mathrm{mmol})$, EDC (0.9 g, 4.76 mmol), DMAP (0.145 g, $1.19 \mathrm{mmol})$ and butan-1-ol (0.353 g, 0.44 $\mathrm{mL}, 4.76 \mathrm{mmol})$ in DCM $(38 \mathrm{~mL})$ at ambient temperature for $24 \mathrm{~h}$ followed by flash column chromatography (Pet.ether/EtOAc $=95 / 05)$ of the crude reaction mixture using silica gel afforded butyl 2-((2-oxo-2-phenylethyl)thio)acetate $1 \mathbf{v}$ as colourless oil ( $0.550 \mathrm{~g}, 87 \%$ yield).

${ }^{8}$ Vedejs, E.; Eberlein, T. H.; Mazur, D. J.; McClure, C. K.; Perry, D. A.; Ruggeri, R.; Schwartz, E.; Stuls, J. S.; Varie, D. L.; Wilde, R. G.; Wittemberger, S. J. Org. Chem. 1986, 51, 1556. 
$\boldsymbol{R}_{\mathbf{f}}($ Pet. ether $/ \mathrm{EtOAc}=90 / 10): 0.43 ;{ }^{1} \mathbf{H}$ NMR $\left(\mathbf{4 0 0} \mathbf{~ M H z}, \mathbf{C D C l}_{3}\right) \delta$ 7.95-7.92 (m, 2H), 7.57-7.53 (m, 1H), 7.46-7.42 (m, 2H), 4.09 (t, J = 6.7 Hz, 2H). 4.00 (s, 2H), 3.29 (s, 2H), 1.61-1.54 (m, 2H), 1.38-1.29 (m, 2H), $0.88(\mathrm{t}, J=7.4 \mathrm{~Hz}, 3 \mathrm{H}) .{ }^{13} \mathbf{C}$ NMR (100 MHz, CDCl $) \delta 194.0,169.9,135.3$, 133.6, 128.7, 128.6, 65,4, 37.7, 33.4, 30.5, 19.1, 13.7. HRMS (ESI) m/z: $[\mathrm{M}+\mathrm{Na}]^{+}$Calcd for $\begin{array}{llllll}\mathrm{C}_{14} \mathrm{H}_{18} \mathrm{NaO}_{3} \mathrm{~S} & 289.0869 ; & \text { Found } & 289.0874 & \text { FTIR } & \text { (cm }\end{array}$ 1) 2961, 1732, 1677, 1451, 1277, 1158, 1123.

\section{Isopropyl 2-((2-oxo-2-phenylethyl)thio)acetate (1w)}

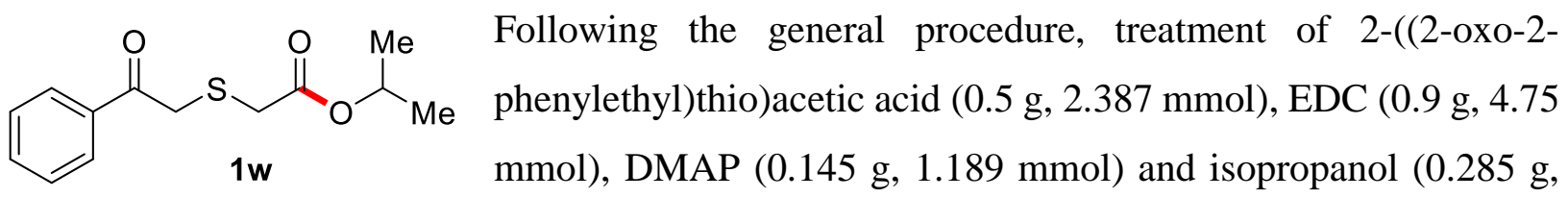

$0.4 \mathrm{~mL}, 4.756 \mathrm{mmol})$ in DCM $(38 \mathrm{~mL})$ at ambient temperature for $24 \mathrm{~h}$ followed by flash column chromatography (Pet.ether/EtOAc $=95 / 05)$ of the crude reaction mixture using silica gel afforded isopropyl 2-((2-oxo-2-phenylethyl)thio)acetate $1 \mathrm{w}$ as pale yellow oil ( $0.485 \mathrm{~g}, 81 \%$ yield).

$\boldsymbol{R} \mathbf{f}\left(\right.$ Pet. ether /EtOAc = 90/10): 0.46; ${ }^{1} \mathbf{H}$ NMR (400 MHz, CDCl $) \delta$ 7.97-7.94 (m, 2H), 7.59-7.55 (m, 1H), 7.48-7.44 (m, 2H), $5.02(\mathrm{sep}, J=6.3 \mathrm{~Hz}, 1 \mathrm{H}), 4.02$ (s, 2H), 3.28 (s, 2H), 1.24 (d, $J=6.2$ $\mathrm{Hz}, 6 \mathrm{H}) .{ }^{13} \mathbf{C}$ NMR (100 MHz, CDCl$) \delta 194.2,169.4,135.5,133.6,128.8,128.7,69.2,37.8$, 33.8, 21.8. HRMS (ESI) $\mathrm{m} / \mathrm{z}$ : $[\mathrm{M}+\mathrm{Na}]^{+} \mathrm{Calcd}$ for $\mathrm{C}_{13} \mathrm{H}_{16} \mathrm{NaO}_{3} \mathrm{~S}$ 275.0712; Found 275.0716. FTIR $\left(\mathbf{c m}^{-1}\right)$ 2981, 1733, 1675, 1450, 1273, 1175, 1104.

\section{Allyl 2-((2-oxo-2-phenylethyl)thio)acetate (1x)}

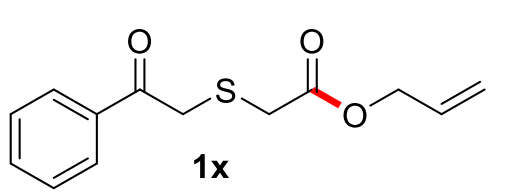

Following the general procedure, treatment of 2-((2-oxo-2phenylethyl)thio)acetic acid( $0.4 \mathrm{~g}, 1.90 \mathrm{mmol}), \mathrm{EDC}(0.728 \mathrm{~g}, 3.8$ mmol), DMAP (0.116 g, $0.95 \mathrm{mmol})$ and prop-2-en-1-ol (0.220 g, $0.26 \mathrm{~mL}, 3.8 \mathrm{mmol})$ in DCM $(30 \mathrm{~mL})$ at ambient temperature for $24 \mathrm{~h}$ followed by flash column chromatography (Pet.ether/EtOAc $=95 / 05)$ of the crude reaction mixture using silica gel afforded allyl 2-((2-oxo-2-phenylethyl)thio)acetate $1 \mathbf{x}$ as pale yellow oil ( $0.4 \mathrm{~g}, 84 \%$ yield).

$\boldsymbol{R}_{\mathbf{f}}($ Pet. ether /EtOAc $=90 / 10): 0.39 ;{ }^{1} \mathbf{H}$ NMR $\left(\mathbf{4 0 0} \mathbf{~ M H z}, \mathbf{C D C l}_{3}\right) \delta$ 7.96-7.94 (m, 2H), 7.59-7.55 (m, 1H), 7.48-7.44 (m, 2H), 5.94-5.85 (m, 1H), 5.35-5.30 (m, 1H), 5.25-5.22(m, 1H), 4.62-4.60 (m, 2H), 4.03 (s, 2H), 3.35 (s, 2H). $\left.{ }^{13} \mathbf{C ~ N M R ~ ( 1 0 0 ~ M H z , ~ C D C l ~}\right) \delta$ 194.1, 169.6, 135.4, 133.6, 
131.7, 128.8, 128.7, 118.9, 66.1, 37.8, 33.4. HRMS (ESI) m/z: $[\mathrm{M}+\mathrm{Na}]^{+} \mathrm{Calcd}$ for $\mathrm{C}_{13} \mathrm{H}_{14} \mathrm{NaO}_{3} \mathrm{~S}$ 273.0556; Found 273.0562. FTIR (cm-1) 2925, 2363, 1734, 1678, 1595, 1277, 1156, 987.

\section{Prop-2-yn-1-yl 2-((2-oxo-2-phenylethyl)thio)acetate (1y)}

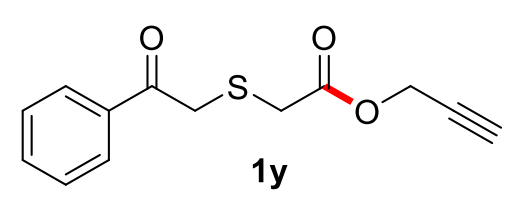

Following the general procedure, treatment of 2-((2-oxo-2phenylethyl)thio)acetic acid( $0.5 \mathrm{~g}, 2.378 \mathrm{mmol})$, EDC $(0.9 \mathrm{~g}$, $4.756 \mathrm{mmol})$, DMAP (0.145 g, $1.189 \mathrm{mmol})$ and prop-2-yn-1-ol

$(0.266 \mathrm{~g}, 0.27 \mathrm{~mL}, 4.756 \mathrm{mmol})$ in DCM $(38 \mathrm{~mL})$ at ambient temperature for $24 \mathrm{~h}$ followed by flash column chromatography (Pet.ether/EtOAc $=95 / 05)$ of the crude reaction mixture using silica gel afforded prop-2-yn-1-yl 2-((2-oxo-2-phenylethyl)thio)acetate 1y as white solid (0.410 g, 69\% yield).

$\boldsymbol{R}_{\mathbf{f}}\left(\mathrm{Pet}\right.$. ether $/ \mathrm{EtOAc}=$ 90/10): 0.38; $\mathrm{mp} 38-40{ }^{\circ} \mathrm{C} ;{ }^{1} \mathbf{H}$ NMR $\left(400 \mathrm{MHz}, \mathbf{C D C l}_{3}\right) \delta$ 7.96-7.94 (m, 2H), 7.60-7.56 (m, 1H), 7.49-7.45 (m, 2H), 4.72 (d, $J=2.4 \mathrm{~Hz}, 2 \mathrm{H}), 4.05(\mathrm{~s}, 2 \mathrm{H}), 3.37$ (s, 2H), $2.48(\mathrm{t}, J=2.4 \mathrm{~Hz}, 1 \mathrm{H}) .{ }^{\mathbf{1 3}} \mathbf{C} \mathbf{N M R}(\mathbf{1 0 0} \mathbf{M H z}, \mathbf{C D C l}) \delta 194.1,169.2,135.4,133.7,128.9,128.7$, 77.2, 75.5, 52.9, 37.8, 33.1. HRMS (ESI) m/z: $[\mathrm{M}+\mathrm{Na}]^{+} \mathrm{Calcd}$ for $\mathrm{C}_{13} \mathrm{H}_{12} \mathrm{NaO}_{3} \mathrm{~S}$ 271.0399; Found 271.0406. FTIR (cm $\left.{ }^{-1}\right)$ 3294, 2949, 2128, 1731, 1674, 1447, 1281, 1120.

\section{Phenethyl 2-((2-oxo-2-phenylethyl)thio)acetate (1z)}

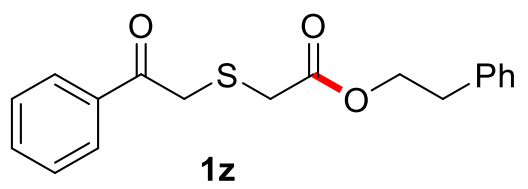

Following the general procedure, treatment of 2-((2-oxo-2phenylethyl)thio)acetic acid (0.5 g, $2.38 \mathrm{mmol})$, EDC (0.911 g, $4.76 \mathrm{mmol})$, DMAP (0.145 g, $1.19 \mathrm{mmol})$ and 2-phenylethan-1ol $(0.581 \mathrm{~g}, 0.57 \mathrm{~mL}, 4.76 \mathrm{mmol})$ in DCM $(38 \mathrm{~mL})$ at ambient temperature for $24 \mathrm{~h}$ followed by flash column chromatography (Pet.ether/EtOAc $=95 / 05)$ of the crude reaction mixture using silica gel afforded phenethyl 2-((2-oxo-2-phenylethyl)thio)acetate $\mathbf{~} \mathbf{z}$ as pale yellow oil ( $0.654 \mathrm{~g}, 87 \%$ yield).

$\boldsymbol{R}_{\mathbf{f}}\left(\right.$ Pet. ether /EtOAc $=$ 90/10): 0.43; ${ }^{\mathbf{1}} \mathbf{H}$ NMR (400 MHz, $\left.\mathbf{C D C l}_{3}\right) \delta$ 7.95-7.93 (m, 2H), 7.61-7.57 (m, 1H), 7.49-7.46 (m, 2H), 7.31-7.26 (m, 2H), 7.23-7.19 (m, 3H), 4.36 (t, J = 7.0 Hz, 2H), 3.95 (s, 2H),3.32 (s, 2H), 2.95 (t, $J=7.0 \mathrm{~Hz}, 2 \mathrm{H}) .{ }^{13} \mathbf{C} \mathbf{~ N M R}\left(\mathbf{1 0 0} \mathbf{~ M H z}, \mathbf{C D C l}_{3}\right) \delta 194.1,169.9,137.6$, 135.4, 133.7, 129.0, 128.8, 128.7, 128.6, 126.7,66.0, 37.8, 35.0, 33.4. HRMS (ESI) m/z: $[\mathrm{M}+\mathrm{Na}]^{+}$ Calcd for $\mathrm{C}_{18} \mathrm{H}_{18} \mathrm{NaO}_{3} \mathrm{~S}$ 337.0869; Found 337.0871. FTIR (cm1) $3028,2957,1725,1672,1600,1450,1272,1157,992$. 


\section{Ethyl 2-((2-(4-chlorophenyl)-2-oxoethyl)thio)acetate (1aa)}<smiles>O=C(CSCC(=O)c1ccccc1)c1ccccc1</smiles>

Following the general procedure, ${ }^{4}$ treatment of 2-((2-oxo-2phenylethyl)thio)acetic acid (1.0 g, $4.76 \mathrm{mmol})$, EDC (1.8 g, 9.52 mmol), DMAP (0.291 g, $2.38 \mathrm{mmol})$ and phenol (0.896 g, $9.52 \mathrm{mmol})$ in DCM $(75 \mathrm{~mL})$ at ambient temperature for $24 \mathrm{~h}$ followed by flash column chromatography (Pet.ether/EtOAc $=95 / 05)$ of the crude reaction mixture using silica gel afforded ethyl phenyl 2((2-oxo-2-phenylethyl)thio)acetate 1aa as colourless oil (1.05 g, 77\% yield).

$\boldsymbol{R}_{\mathbf{f}}\left(\right.$ Pet. ether $/ \mathrm{EtOAc}=$ 90/10): 0.31; ${ }^{1} \mathbf{H}$ NMR (400 MHz, CDCl $) \delta$ 7.99-7.97 (m, 2H), 7.61-7.57 (m, 1H), 7.49-7.45 (m, 2H), 7.40-7.36 (m, 2H), 7.26-7.22 (m, 1H), 7.14-7.11 (m, 2H), 4.12 (s, 2H), 3.57 (s, 2H). ${ }^{13}$ C NMR (100 MHz, CDCl $) \delta 194.1,168.5,150.6,135.4,133.7,129.6,128.9$, 128.7, 126.2, 121.4,37.8, 33.5. HRMS (ESI) m/z: $[\mathrm{M}+\mathrm{Na}]^{+}$Calcd for $\mathrm{C}_{16} \mathrm{H}_{14} \mathrm{NaO}_{3} \mathrm{~S} 309.0556$; Found 309.0560. FTIR (cm-1) 3464, 2920, 1758, 1673, 1592, 1488, 1280, 1114.

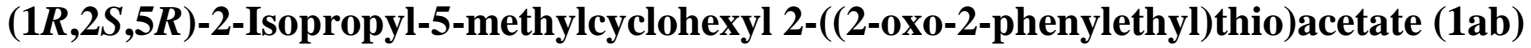<smiles>CC(C)[C@H]1CC[C@@H](C)C[C@H]1OC(=O)CSCC(=O)c1ccccc1</smiles>
Following the general procedure, treatment of 2-((2-oxo-2phenylethyl)thio)acetic acid (0.5 g, $2.38 \mathrm{mmol})$, EDC (0.9 g, $4.75 \mathrm{mmol})$, DMAP (0.145 g, $1.19 \mathrm{mmol})$ and (1R,2S,5R)-2isopropyl-5-methylcyclohexan-1-ol (0.742 $\mathrm{g}, 4.75 \mathrm{mmol})$ in

DCM $(38 \mathrm{~mL})$ at ambient temperature for $24 \mathrm{~h}$ followed by flash column chromatography (Pet.ether/EtOAc $=95 / 05)$ of the crude reaction mixture using silica gel afforded (1R,2S,5R)-2-isopropyl-5-methylcyclohexyl 2-((2-oxo-2-phenylethyl)thio)acetate 1ab as pale yellow oil ( $0.650 \mathrm{~g}, 79 \%$ yield $)$.

$\boldsymbol{R}_{\mathbf{f}}\left(\right.$ Pet. ether $/$ EtOAc $=$ 90/10): 0.35; ${ }^{1} \mathbf{H}$ NMR (400 MHz, $\left.\mathbf{C D C l}_{3}\right) \delta$ 7.98-7.96 (m, 2H), 7.61-7.57 $(\mathrm{m}, 1 \mathrm{H}), 7.50-7.48(\mathrm{~m}, 2 \mathrm{H}), 4.71\left(\mathrm{td}, J_{1}=10.9 \mathrm{~Hz}, J_{2}=4.4 \mathrm{~Hz}, 1 \mathrm{H}\right), 4.07-3.99(\mathrm{~m}, 2 \mathrm{H}), 3.31(\mathrm{~s}$, 2H), 2.02-1.97 (m, 1H), 1.93-1.85 (m, 1H), 1.69-1.65 (m, 2H), 1.53-1.43 (m, 1H), 1.41-1.35 (m, 1H), 1.09-0.87 (m, 9H), $0.75(\mathrm{~d}, J=7.0 \mathrm{~Hz}, 3 \mathrm{H}) .{ }^{13} \mathbf{C}$ NMR (100 MHz, CDCl3) $\delta$ 194.2, 169.6, 135.6, 133.7, 128.9, 128.8,75.7, 47.1, 40.8, 37.8, 34.3, 33.8, 31.5, 26.3, 23.5, 22.1, 20.9, 16.4. HRMS (ESI) m/z: [M+Na $]^{+}$Calcd for $\mathrm{C}_{20} \mathrm{H}_{28} \mathrm{NaO}_{3} \mathrm{~S}$ 371.1651; Found 371.1657. FTIR (cm1) $2957,2866,1727,1678,1451,1277,1172$. 


\section{(E)-3,7-dimethylocta-2,6-dien-1-yl 2-((2-oxo-2-phenylethyl)thio)acetate (1ac)}

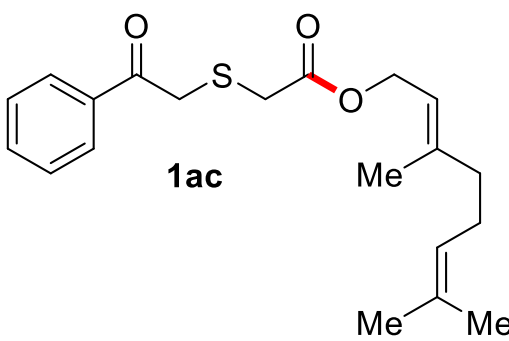

Following the general procedure, treatment of 2-((2-oxo-2phenylethyl)thio)acetic acid (0.5 g, $2.38 \mathrm{mmol})$, EDC (0.911 g, $4.76 \mathrm{mmol})$, DMAP (0.145 $\mathrm{g}, 1.19 \mathrm{mmol})$ and $(E)-3,7-$ dimethylocta-2,6-dien-1-ol $(0.720 \mathrm{~g}, 0.57 \mathrm{~mL}, 4.76 \mathrm{mmol})$ in DCM (38 mL) at ambient temperature for $24 \mathrm{~h}$ followed by flash column chromatography $($ Pet.ether/EtOAc $=95 / 05)$ of the crude reaction mixture using silica gel afforded (E)-3,7-dimethylocta-2,6-dien-1-yl 2-((2-oxo-2phenylethyl)thio)acetate 1ac as pale yellow oil (0.502 g, 61\% yield).

$\boldsymbol{R}_{\mathbf{f}}($ Pet. ether $/$ EtOAc $=95 / 05)$ : 0.49; ${ }^{1} \mathbf{H}$ NMR (400 MHz, CDCl $) \delta$ 7.97-7.95 (m, 2H), 7.60-7.56 (m, 1H), 7.49-7.45 (m, 2H), 5.35-5.31 (m, 1H), 5.08-5.05 (m, 1H), $4.64(\mathrm{~d}, J=7.2 \mathrm{~Hz}, 2 \mathrm{H}), 4.04(\mathrm{~s}$, 2H), 3.33 (s, 2H), 2.11-2.01 (m, 4H), 1.69 (s, 3H), 1.67 (s, 3H), 1.59 (s, 3H). ${ }^{13}$ C NMR (100 MHz, CDCl3) $\delta 194.2,170.0,143.1,135.5,133.7,132.0,128.8,128.7,123.8,117.9,62.5,39.6,37.8$, 33.5, 26.4, 25.8, 17.8, 16.6. HRMS (ESI) m/z: [M+Na] ${ }^{+}$Calcd for $\mathrm{C}_{20} \mathrm{H}_{26} \mathrm{NaO}_{3} \mathrm{~S} 369.1495$; Found 369.1496. FTIR (cm-1) 2920, 1731, 1677, 1447, 1278, 1155, 1121.

$(3 R, 8 S, 9 S, 10 R, 13 R, 14 S, 17 R)-10,13-D i m e t h y l-17-((R)-6-m e t h y l h e p t a n-2$

2,3,4,7,8,9,10,11,12,13,14,15,16,17-tetradecahydro- $1 H$-cyclopenta[a]phenanthren-3-yl 2-((2oxo-2-phenylethyl)thio)acetate (1ad)

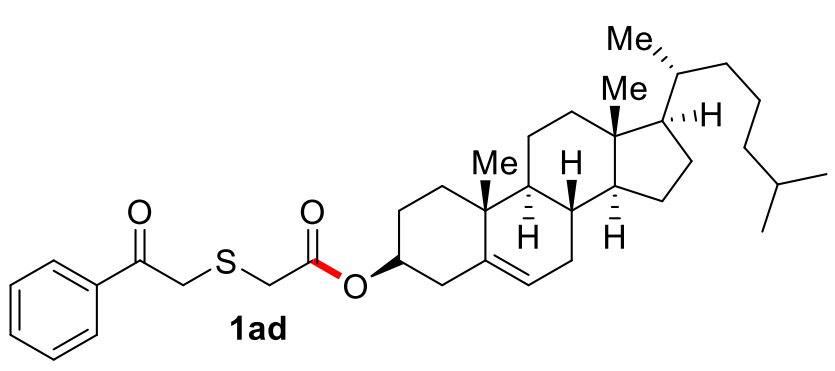

Following the general procedure, treatment of 2-((2-oxo-2-phenylethyl)thio)acetic acid (0.5 g, $2.38 \mathrm{mmol})$, EDC (0.9 g, $4.75 \mathrm{mmol})$, DMAP (0.145 g, $1.18 \mathrm{mmol})$ and cholesterol $(1.8 \mathrm{~g}, 4.75 \mathrm{mmol})$ in $\mathrm{DCM}(38 \mathrm{~mL})$ at ambient temperature for $24 \mathrm{~h}$ followed by flash column chromatography $($ Pet.ether/EtOAc $=95 / 05)$ of the crude reaction mixture using silica gel afforded $\quad(3 R, 8 S, 9 S, 10 R, 13 R, 14 S, 17 R)-10,13$-dimethyl-17-((R)-6-methylheptan-2yl)2,3,4,7,8,9,10,11,12,13,14,15,16,17-tetradecahydro-1H-cyclopenta[a]phenanthren-3-yl 2-((2oxo-2-phenylethyl)thio)acetate 1ad as white solid (0.825 g, 60\% yield).

$\boldsymbol{R}_{\mathbf{f}}($ Pet. ether $/$ EtOAc $=90 / 10): 0.47 ; \mathrm{mp} 103-105{ }^{\circ} \mathrm{C} ;{ }^{1} \mathbf{H}$ NMR (400 MHz, CDCl $) \delta$ 7.98-7.96 $(\mathrm{m}, 2 \mathrm{H}), 7.60-7.57(\mathrm{~m}, 1 \mathrm{H}), 7.49-7.46(\mathrm{~m}, 2 \mathrm{H}), 5.38-5.36(\mathrm{~m}, 1 \mathrm{H}), 4.68-4.60(\mathrm{~m}, 1 \mathrm{H}), 4.03(\mathrm{~s}, 2 \mathrm{H})$, 
3.30 (s, 2H), 2.34-2.32 (m, 2H), 2.02-1.95 (m, 2H), 1.89-1.80 (m, 3H), 1.64-1.08 (m, 18H), 1.040.85 (m, 15H), 0.67 (s, 3H). ${ }^{13} \mathbf{C}$ NMR (100 MHz, CDCl3) $\delta$ 194.2, 169.4, 139.5, 135.6, 133.7, 128.9, 128.8, 123.0,75.5, 56.8, 56.3, 50.1, 42.5, 39.9, 39.7, 38.1, 37.8, 37.1, 36.7, 36.3, 35.9, 33.8, 32.0, 32.0, 28.4, 28.2, 27.8, 24.4, 24.0, 23.0, 22.7, 21.2, 19.4, 18.9, 12.0. HRMS (ESI) m/z: $[\mathrm{M}+\mathrm{Na}]^{+}$Calcd for $\mathrm{C}_{37} \mathrm{H}_{54} \mathrm{NaO}_{3} \mathrm{~S}$ 601.3686; Found 601.3694. FTIR (cm-1) 2935, 2869, 1733, $1671,1291,1162,1005$

(R)-2-((Tert-butoxycarbonyl)amino)-3-phenylpropyl 2-((2-0xo-2 phenylethyl)thio) acetate(1ae)<smiles>NC(COC(=O)CSCC(=O)c1ccccc1)Cc1ccccc1</smiles>
Following the general procedure, treatment of 2-((2-oxo-2phenylethyl)thio)acetic acid( $0.2 \mathrm{~g}, 0.95 \mathrm{mmol}), \mathrm{EDC}(0.365$ $\mathrm{g}, 1.90 \mathrm{mmol})$, DMAP (0.058 g, $0.475 \mathrm{mmol})$ and tert-butyl $(R)$-(1-hydroxy-3-phenylpropan-2-yl)carbamate $(0.478 \mathrm{~g}$, $1.90 \mathrm{mmol})$ in DCM $(15 \mathrm{~mL})$ at ambient temperature for $24 \mathrm{~h}$ followed by flash column chromatography (Pet.ether/EtOAc $=90 / 10)$ of the crude reaction mixture using silica gel afforded (R)-2-((tert-butoxycarbonyl)amino)-3-phenylpropyl 2-((2-oxo-2-phenylethyl)thio)acetate 1ae as white solid ( $0.4 \mathrm{~g}, 95 \%$ yield $)$.

$\boldsymbol{R}_{\mathbf{f}}($ Pet. ether $/$ EtOAc $=90 / 10): 0.33 ; \mathrm{mp} 72-74{ }^{\circ} \mathrm{C} ;{ }^{1} \mathbf{H}$ NMR $\left(400 \mathbf{~ M H z}, \mathbf{C D C l}_{3}\right) \delta$ 7.99-7.97 (m, $2 \mathrm{H}), 7.61-7.57(\mathrm{~m}, 1 \mathrm{H}), 7.50-7.46(\mathrm{~m}, 2 \mathrm{H}), 7.31-7.26(\mathrm{~m}, 2 \mathrm{H}), 7.23-7.19(\mathrm{~m}, 3 \mathrm{H}), 4.91-4.89(\mathrm{~m}$, 1H), 4.20-4.02 (m, 5H), 3.36 (s, 2H), 2.92-2.79 (m, 2H), 1.40 (s, 9H). ${ }^{13}$ C NMR (100 MHz, $\left.\mathbf{C D C l}_{3}\right) \delta 194.1,169.6,155.3,137.3,135.3,133.6,129.3,128.8,128.7,128.6,126.7,79.5,65.8$, 50.7, 37.8, 37.6, 33.3, 28.4. HRMS (ESI) m/z: $[\mathrm{M}+\mathrm{Na}]^{+}$Calcd for $\mathrm{C}_{24} \mathrm{H}_{29} \mathrm{NNaO}_{5} \mathrm{~S} 466.1659$; Found 466.1660. FTIR (cm $\left.\mathbf{c m}^{-1}\right)$ 3371, 2975, 1709, 1599, 1518, 1278, 1166.

\section{$N, N$-Dimethyl-2-((2-oxo-2-phenylethyl)thio)acetamide(1af)}

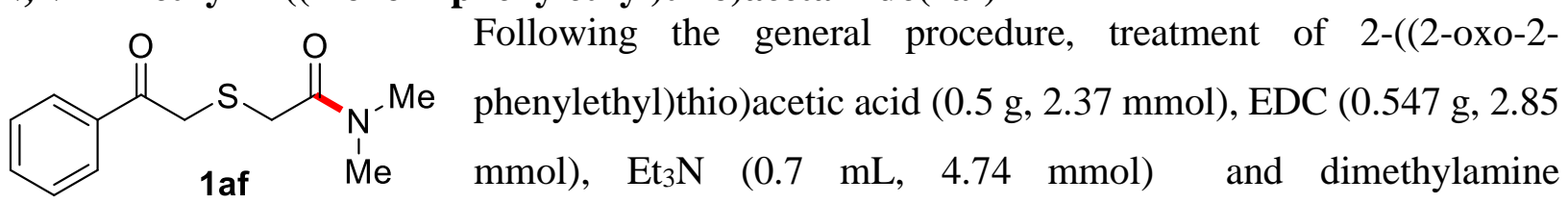

hydrochloride $(0.232 \mathrm{~g}, 2.85 \mathrm{mmol})$ in DCM $(7 \mathrm{~mL})$ at ambient temperature for overnight followed by flash column chromatography (Pet.ether/EtOAc $=95 / 05$ ) of the crude reaction mixture using 
silica gel afforded $N, N$-dimethyl-2-((2-oxo-2-phenylethyl)thio)acetamide 1af as light yellow solid (0.450 g, 80\% yield).

$\boldsymbol{R}_{\mathbf{f}}($ Pet. ether $/ \mathrm{EtOAc}=90 / 10): 0.40 ; \mathrm{mp} 47-49^{\circ} \mathrm{C} ;{ }^{1} \mathbf{H}$ NMR (400 MHz, CDCl3) $\delta$ 7.96-7.94 (m, 2H), 7.57-7.53 (m, 1H), 7.46-7.42 (m, 2H), $4.05(\mathrm{~s}, 2 \mathrm{H}), 3.40(\mathrm{~s}, 2 \mathrm{H}), 3.03(\mathrm{~s}, 3 \mathrm{H}), 2.92(\mathrm{~s}, 3 \mathrm{H})$. ${ }^{13}$ C NMR (100 MHz, CDCl3) $\delta$ 194.6, 168.6, 135.6, 133.5, 128.8, 128.7,38.1, 37.8, 35.8, 33.5. HRMS (ESI) m/z: [M+Na] ${ }^{+}$Calcd for $\mathrm{C}_{12} \mathrm{H}_{15} \mathrm{NNaO}_{2} \mathrm{~S}$ 260.0716; Found 260.0721. FTIR (cm1) $2936,1684,1637,1448,1395,1278,1201,1137$.

\section{$N$-Methoxy- $N$-methyl-2-((2-oxo-2-phenylethyl)thio)acetamide (1ag)}

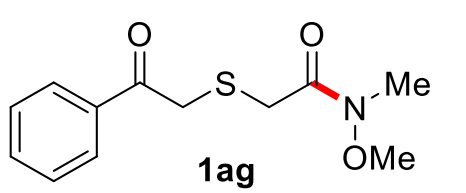

Following the general procedure, treatment of 2-((2-oxo-2phenylethyl)thio)acetic acid (0.5 g, $2.37 \mathrm{mmol})$, EDC (0.547 g, 2.85 $\mathrm{mmol}), \mathrm{Et}_{3} \mathrm{~N}(0.7 \mathrm{~mL}, 4.74 \mathrm{mmol})$ and N,O-dimethylhydroxylamine hydrochloride $(0.277 \mathrm{~g}, 2.85 \mathrm{mmol})$ in DCM $(7 \mathrm{~mL})$ at ambient temperature for overnight followed by flash column chromatography (Pet.ether/EtOAc $=85 / 15)$ of the crude reaction mixture using silica gel afforded $N$-methoxy- $N$-methyl-2-((2-oxo-2-phenylethyl)thio)acetamide 1ag as light yellow semi-solid $(0.375 \mathrm{~g}, 63 \%$ yield $)$.

$\boldsymbol{R}_{\mathbf{f}}\left(\right.$ Pet. ether $/ \mathrm{EtOAc}=$ 90/10): 0.15; ${ }^{1} \mathbf{H}$ NMR (400 MHz, CDCl $) \delta$ 7.97-7.95 (m, 2H), 7.58-7.54 (m, 1H), 7.47-7.43 (m, 2H), $4.08(\mathrm{~s}, 2 \mathrm{H}), 3.71(\mathrm{~s}, 3 \mathrm{H}), 3.49$ (s, 2H), 3.19 (s, 3H). ${ }^{13} \mathrm{C}$ NMR (100 $\left.\mathbf{M H z}_{\mathbf{1}} \mathbf{C D C l}_{3}\right) \delta 194.2,169.9,135.4,133.2,128.5,128.4,61.3,37.7,32.1,31.5$. HRMS (ESI) m/z: $[\mathrm{M}+\mathrm{Na}]^{+}$Calcd for $\mathrm{C}_{12} \mathrm{H}_{15} \mathrm{NNaO}_{3} \mathrm{~S}$ 276.0665; Found 276.0670. FTIR (cm $\left.{ }^{-1}\right)$ 2937, 1677, 1647, 1592, 1449, 1278, 1201, 997.

\section{Ethyl 2-((2-oxocyclohexyl)thio)acetate (1ah)}

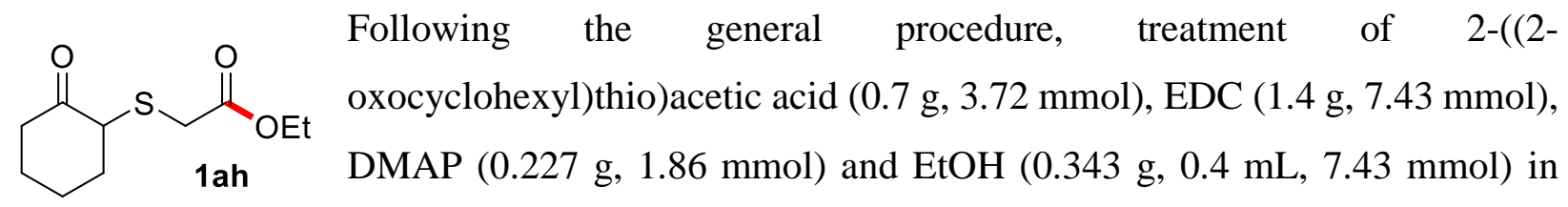

DCM $(59 \mathrm{~mL})$ at ambient temperature for $24 \mathrm{~h}$ followed by flash column chromatography (Pet.ether/EtOAc $=95 / 05)$ of the crude reaction mixture using silica gel afforded ethyl 2-((2oxocyclohexyl)thio)acetate 1ah as pale yellow oil (0.510 g, 64\% yield).

$\boldsymbol{R}_{\mathbf{f}}\left(\right.$ Pet. ether $/ \mathrm{EtOAc}$ 90/10): 0.31; ${ }^{1} \mathbf{H}$ NMR (400 MHz, CDCl3) $\delta 4.16(\mathrm{q}, J=7.1 \mathrm{~Hz}, 2 \mathrm{H})$, 3.58-3.56 (m, 1H), 3.29 (d, $J=15.4 \mathrm{~Hz}, 1 \mathrm{H}), 3.20$ (d, $J=15.4 \mathrm{~Hz}, 1 \mathrm{H}), 2.92-2.84(\mathrm{~m}, 1 \mathrm{H}), 2.30-$ 
$2.19(\mathrm{~m}, 2 \mathrm{H}), 2.02-1.69(\mathrm{~m}, 5 \mathrm{H}), 1.26(\mathrm{t}, J=7.1 \mathrm{~Hz}, 3 \mathrm{H}) .{ }^{13} \mathbf{C} \mathbf{~ N M R}\left(\mathbf{1 0 0} \mathbf{M H z}, \mathbf{C D C l}_{3}\right) \delta 207.6$, 170.1, 61.6, 52.6, 38.5, 33.2, 33.0, 27.0, 22.5, 14.2. HRMS (ESI) m/z: $[\mathrm{M}+\mathrm{Na}]^{+}$Calcd for $\begin{array}{lllll}\mathrm{C}_{10} \mathrm{H}_{16} \mathrm{NaO}_{3} \mathrm{~S} & 239.0712 ; & \text { Found } & 239.0718 . & \text { FTIR }\end{array}$ 1) $2936,1778,1736,1446,1298,1152,1121,1028$.

\section{Ethyl 2-((2-oxocycloheptyl)thio)acetate (1ai)}<smiles>CCOC(=O)CSC1CCCCCC1=O</smiles>

Following the general procedure, treatment of 2-((2oxocycloheptyl)thio)acetic acid (0.607 g, $3.0 \mathrm{mmol})$, EDC (1.15 g, 6.0 mmol), DMAP (0.183 g, $0.5 \mathrm{mmol})$ and EtOH $(0.276 \mathrm{~g}, 0.35 \mathrm{~mL}, 6.0$ $\mathrm{mmol})$ in DCM $(50 \mathrm{~mL})$ at ambient temperature for $24 \mathrm{~h}$ followed by flash column chromatography (Pet.ether/EtOAc $=95 / 05)$ of the crude reaction mixture using silica gel afforded ethyl 2-((2-oxocycloheptyl)thio)acetate 1ai as pale yellow oil ( $0.585 \mathrm{~g}, 85 \%$ yield). $\boldsymbol{R}_{\mathbf{f}}($ Pet. ether $/$ EtOAc $=95 / 05)$ : 0.38; ${ }^{1} \mathbf{H}$ NMR (400 MHz, $\left.\mathbf{C D C l}_{3}\right) \delta 4.20-4.12(\mathrm{~m}, 2 \mathrm{H}), 3.42-3.38$ (m, 1H), $3.32(\mathrm{~d}, J=15.7 \mathrm{~Hz}, 1 \mathrm{H}), 3.15(\mathrm{~d}, J=15.7 \mathrm{~Hz}, 1 \mathrm{H}), 2.84-2.77(\mathrm{~m}, 1 \mathrm{H}), 2.38-2.33(\mathrm{~m}$, 1H), 2.20-2.13 (m, 1H), 1.96-1.79 (m, 3H), 1.49-1.33 (m, 3H), 1.27-1.21 (m, 4H). ${ }^{\mathbf{1 3}} \mathbf{C}$ NMR (100 MHz, CDCl $) \delta 208.4,169.9,61.6,53.2,39.4,33.7,30.3,29.7,27.3,25.8,14.2$. HRMS (ESI) m/z: $[\mathrm{M}+\mathrm{Na}]^{+}$Calcd for $\mathrm{C}_{11} \mathrm{H}_{18} \mathrm{NaO}_{3} \mathrm{~S}$ 253.0869; Found 253.0872. FTIR (cm-1) 2932, 2856, 1736, 1693, 1450, 1297, 1163, 1028.

\section{Ethyl 2-((1-oxo-2,3-dihydro-1 $H$-inden-2-yl)thio)acetate (1aj)}

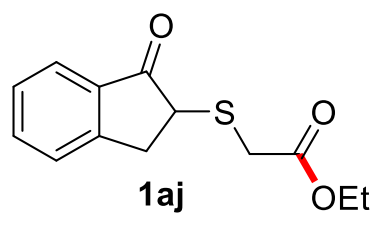

Following the general procedure, treatment of 2-((1-oxo-2,3-dihydro- $1 \mathrm{H}$ inden-2-yl)thio)acetic acid (1.0 g, $4.49 \mathrm{mmol})$, EDC (1.7 g, $8.99 \mathrm{mmol})$, DMAP $(0.274 \mathrm{~g}, 2.25 \mathrm{mmol})$ and EtOH $(0.415 \mathrm{~g}, 0.53 \mathrm{~mL}, 8.99 \mathrm{mmol})$ in DCM $(71 \mathrm{~mL})$ at ambient temperature for $24 \mathrm{~h}$ followed by flash column chromatography (Pet.ether/EtOAc $=95 / 05)$ of the crude reaction mixture using silica gel afforded ethyl 2-((1-oxo-2,3-dihydro- $1 H$-inden-2-yl)thio)acetate 1aj as pale yellow oil ( $0.8 \mathrm{~g}, 71 \%$ yield).

$\boldsymbol{R}_{\mathbf{f}}($ Pet. ether $/ \mathrm{EtOAc}=90 / 10): 0.35 ;{ }^{1} \mathbf{H}$ NMR $\left(400 \mathrm{MHz}, \mathbf{C D C l}_{3}\right) \delta 7.74(\mathrm{~d}, J=7.6 \mathrm{~Hz}, 1 \mathrm{H})$, 7.60-7.56 (m, 1H), 7.41-7.35 (m, 2H), $4.17(\mathrm{q}, J=7.1 \mathrm{~Hz}, 2 \mathrm{H}), 3.89-3.83(\mathrm{~m}, 2 \mathrm{H}), 3.59$ (dd, $J_{l}=$ $\left.17.8 \mathrm{~Hz}, J_{2}=7.9 \mathrm{~Hz}, 1 \mathrm{H}\right), 3.33(\mathrm{~d}, J=15.6 \mathrm{~Hz}, 1 \mathrm{H}), 2.92\left(\mathrm{dd}, J_{l}=17.8 \mathrm{~Hz}, J_{2}=3.0 \mathrm{~Hz}, 1 \mathrm{H}\right), 1.25$ $(\mathrm{t}, J=7.1 \mathrm{~Hz}, 3 \mathrm{H}) .{ }^{13} \mathbf{C}$ NMR (100 MHz, CDCl$) \delta 202.9,170.1,151.9,135.3,135.0,128.0,126.4$, 
124.7,61.5, 44.7, 34.1, 32.6, 14.2. HRMS (ESI) m/z: [M+Na] ${ }^{+}$Calcd for $\mathrm{C}_{13} \mathrm{H}_{14} \mathrm{NaO}_{3} \mathrm{~S} 273.0556$; Found 273.0559. FTIR $\left(\mathbf{c m}^{-1}\right)$ 2983, 2927, 1708, 1608, 1299, 1272, 1028

\section{Ethyl 2-((1-oxo-1,2,3,4-tetrahydronaphthalen-2-yl)thio)acetate (1ak)}<smiles>CCOC(=O)CSC1CCc2ccccc2C1=O</smiles>

Following the general procedure, treatment of 2-((1-oxo-1,2,3,4-

tetrahydronaphthalen-2-yl)thio)acetic acid (0.7 g, $2.96 \mathrm{mmol})$, EDC (1.13 g, $5.92 \mathrm{mmol})$, DMAP (0.181 g, $1.48 \mathrm{mmol})$ and EtOH (0.273 g, $0.35 \mathrm{~mL}, 5.92 \mathrm{mmol})$ in DCM $(47 \mathrm{~mL})$ at ambient temperature for $24 \mathrm{~h}$ followed by flash column chromatography (Pet.ether/EtOAc $=95 / 05)$ of the crude reaction mixture using silica gel afforded ethyl 2-((1-oxo-1,2,3,4-tetrahydronaphthalen-2-yl)thio)acetate 1ak as light yellow solid ( $0.650 \mathrm{~g}$, $80 \%$ yield).

$\boldsymbol{R} \mathbf{f}($ Pet. ether $/$ EtOAc $=90 / 10): 0.47 ; \mathrm{mp} 53-55^{\circ} \mathrm{C} ;{ }^{1} \mathbf{H}$ NMR $\left(400 \mathbf{~ M H z}, \mathbf{C D C l}_{3}\right) \delta 8.06(\mathrm{~d}, J=$ $7.8 \mathrm{~Hz}, 1 \mathrm{H}), 7.48-7.44(\mathrm{~m}, 1 \mathrm{H}), 7.31(\mathrm{t}, J=7.5 \mathrm{~Hz}, 1 \mathrm{H}), 7.21(\mathrm{~d}, J=7.6 \mathrm{~Hz}, 1 \mathrm{H}), 4.19(\mathrm{q}, J=7.1$ Hz, 2H), 3.83 (t, $J=4.5 \mathrm{~Hz}, 1 \mathrm{H}), 3.53$ (d, $J=15.7 \mathrm{~Hz}, 1 \mathrm{H}), 3.27$ (d, $J=15.7 \mathrm{~Hz}, 1 \mathrm{H}), 3.22-3.14$ (m, 1H), $2.86\left(\mathrm{dt}, J_{l}=17.1 \mathrm{~Hz}, J_{2}=4.5 \mathrm{~Hz}, 1 \mathrm{H}\right), 2.57-2.49(\mathrm{~m}, 1 \mathrm{H}), 2.35-2.28(\mathrm{~m}, 1 \mathrm{H}), 1.27(\mathrm{t}, J$ $=7.1 \mathrm{~Hz}, 3 \mathrm{H}) .{ }^{13} \mathbf{C}$ NMR (100 MHz, $\left.\mathbf{C D C l}_{3}\right) \delta 1$ 92.9, 170.0, 143.0, 133.7, 130.8, 128.7, 128.3, 127.0,61.6, 48.2, 32.5, 28.5, 26.1, 14.2. HRMS (ESI) m/z: $[\mathrm{M}+\mathrm{Na}]^{+}$Calcd for $\mathrm{C}_{14} \mathrm{H}_{16} \mathrm{NaO}_{3} \mathrm{~S}$ 287.0712; Found 287.0713. FTIR ( (cm $\left.^{-1}\right)$ 2983, 1734, 1675, 1600, 1452, 1301, 1152, 1028.

\section{Ethyl 2-((2-oxopropyl)thio)acetate(1al)}

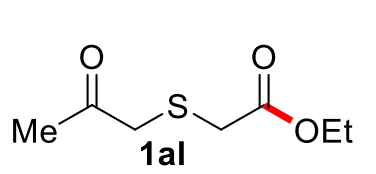

Following the general procedure, treatment of 2-((2-oxopropyl)thio)acetic acid (0.7 g, $4.72 \mathrm{mmol})$, EDC (1.8 g, $9.45 \mathrm{mmol})$, DMAP (0.288 g, 2.36 $\mathrm{mmol})$ and $\mathrm{EtOH}(0.435 \mathrm{~g}, 0.55 \mathrm{~mL}, 9.45 \mathrm{mmol})$ in DCM $(75 \mathrm{~mL})$ at ambient temperature for $24 \mathrm{~h}$ followed by flash column chromatography (Pet.ether/EtOAc $=$ 95/05) of the crude reaction mixture using silica gel afforded ethyl 2-((2-oxopropyl)thio)acetate 1al as pale yellow oil $(0.750 \mathrm{~g}, 90 \%$ yield $)$.

$\boldsymbol{R}_{\mathbf{f}}($ Pet. ether $/$ EtOAc $=90 / 10)$ : 0.31; ${ }^{\mathbf{1}} \mathbf{H}$ NMR $\left(400 \mathbf{~ M H z}, \mathbf{C D C l}_{3}\right) \delta 4.13(\mathrm{q}, J=7.1 \mathrm{~Hz}, 2 \mathrm{H}), 3.38$ (s, 2H), 3.20 (s, 2H), 2.24 (s, 3H), 1.23 (t, $J=7.1 \mathrm{~Hz}, 3 \mathrm{H}) .{ }^{13} \mathbf{C}$ NMR (100 MHz, CDCl $) \delta 203.0$, 169.8,61.5, 42.2, 33.4, 28.3, 14.1. HRMS (ESI) m/z: $[\mathrm{M}+\mathrm{Na}]^{+} \mathrm{Calcd}$ for $\mathrm{C}_{7} \mathrm{H}_{12} \mathrm{NaO}_{3} \mathrm{~S}$ 199.0399; Found 199.0402. FTIR (cm $\left.\mathbf{c m}^{-1}\right)$ 2984, 1735, 1701, 1299, 1154, 1124, 1028. 


\section{Synthesis and Characterization of Functionalized Enol Ethers}

Ethyl-2-(phenylthio)-2-((1-phenylvinyl)oxy)acetate (3a)

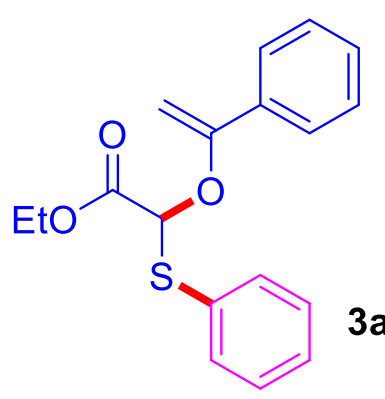

Following the general procedure, treatment of 2-(trimethylsilyl) phenyltrifluoromethanesulfonate $2 \mathbf{a}(0.179 \mathrm{~g}, 146 \mu \mathrm{L}, 0.6 \mathrm{mmol})$ with ethyl 2-((2-oxo-2-phenylethyl)thio)acetate $1 \mathrm{a}(0.119 \mathrm{~g}, 0.5 \mathrm{mmol})$ in the presence of KF (0.070 g, $1.2 \mathrm{mmol})$ and 18-crown-6 (0.317 g, $1.2 \mathrm{mmol})$ in $\mathrm{THF}(2.0 \mathrm{~mL})$ at $25{ }^{\circ} \mathrm{C}$ for $1 \mathrm{~h}$ followed by flash column chromatography (Pet.ether/EtOAc $=97 / 03)$ of the crude reaction mixture using silica gel afforded ethyl-2-(phenylthio)-2-((1-phenylvinyl)oxy)acetate 3a as a colourless oil ( $0.143 \mathrm{~g}, 91 \%$ yield $)$.

$\boldsymbol{R}_{\mathbf{f}}($ Pet. ether $/ \mathrm{EtOAc}=95 / 05): 0.46 ;{ }^{1} \mathbf{H}$ NMR (400 MHz, CDCl3) $\delta$ 7.67-7.63 (m, 2H), 7.60-7.55 (m, 2H), 7.39-7.30 (m, 6H), $5.73(\mathrm{~s}, 1 \mathrm{H}), 4.91(\mathrm{~d}, J=3.7 \mathrm{~Hz}, 1 \mathrm{H}), 4.34(\mathrm{~d}, J=3.7 \mathrm{~Hz}, 1 \mathrm{H}), 4.22-$ $4.11(\mathrm{~m}, 2 \mathrm{H}), 1.20(\mathrm{t}, J=7.1 \mathrm{~Hz}, 3 \mathrm{H}) .{ }^{13} \mathbf{C} \mathbf{N M R}(\mathbf{1 0 0} \mathbf{M H z}, \mathbf{C D C l} 3) \delta 166.9,157.9,135.4,134.6$, 130.8, 129.1, 129.05, 129.0, 128.3, 125.8, 86.9, 81.3, 61.9, 14.1. HRMS (ESI) m/z: [M+Na $]^{+}$ Calcd for $\mathrm{C}_{18} \mathrm{H}_{18} \mathrm{NaO}_{3} \mathrm{~S}$ 337.0869; Found 337.0874. FTIR (cm $^{-}$ 1) $2933,2347,1753,1733,1620,1442,1182,1119$.

\section{Ethyl-2-((1-(4-methoxyphenyl)vinyl)oxy)-2-(phenylthio)acetate (3b)}

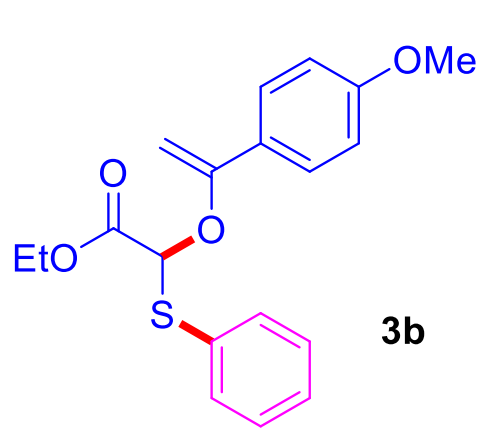

Following the general procedure, treatment of 2-(trimethylsilyl) phenyltrifluoromethanesulfonate $2 \mathbf{a}(0.179 \mathrm{~g}, 146 \mu \mathrm{L}, 0.6 \mathrm{mmol})$ with ethyl 2-((2-(4-methoxyphenyl)-2-oxoethyl)thio)acetate $\mathbf{1 b}$ $(0.134 \mathrm{~g}, 0.5 \mathrm{mmol})$ in the presence of $\mathrm{KF}(0.070 \mathrm{~g}, 1.2 \mathrm{mmol})$ and 18-crown-6 (0.317 g, $1.2 \mathrm{mmol})$ in THF $(2.0 \mathrm{~mL})$ at $25^{\circ} \mathrm{C}$ for $3 \mathrm{~h}$ followed by flash column chromatography (Pet.ether/EtOAc = 96/04) of the crude reaction mixture using silica gel afforded ethyl2-((1-(4-methoxyphenyl)vinyl)oxy)-2-(phenylthio)acetate $\mathbf{3 b}$ as a colourless oil (0.125 g, 73\% yield).

$\boldsymbol{R}_{\mathbf{f}}\left(\right.$ Pet. ether $/$ EtOAc $=$ 95/05): 0.31; ${ }^{1} \mathbf{H}$ NMR (400 MHz, CDCl3) $\delta$ 7.59-7.55 (m, 4H), 7.37-7.29 (m, 3H), 6.89-6.86 (m, 2H), $5.71(\mathrm{~s}, 1 \mathrm{H}), 4.78(\mathrm{~d}, J=3.6 \mathrm{~Hz}, 1 \mathrm{H}), 4.24(\mathrm{~d}, J=3.6 \mathrm{~Hz}, 1 \mathrm{H}), 4.19$ $4.12(\mathrm{~m}, 2 \mathrm{H}), 3.82(\mathrm{~s}, 3 \mathrm{H}), 1.19(\mathrm{t}, J=7.1 \mathrm{~Hz}, 3 \mathrm{H}) .{ }^{13} \mathbf{C}$ NMR (100 MHz, CDCl$) \delta$ 167.0, 160.3, 
157.8, 134.5, 130.9, 129.1, 128.1, 127.2, 113.7, 85.4, 81.2, 61.9, 55.4, 14.1. HRMS (ESI) m/z; $[\mathrm{M}+\mathrm{Na}]^{+}$Calcd for $\mathrm{C}_{19} \mathrm{H}_{20} \mathrm{NaO}_{4} \mathrm{~S}$ 367.0975; Found 367.0980. FTIR (cm1) $3433,2934,1736,1672,1599,1257,1174,1024$.

Ethyl 2-(phenylthio)-2-((1-(p-tolyl)vinyl)oxy)acetate (3c)

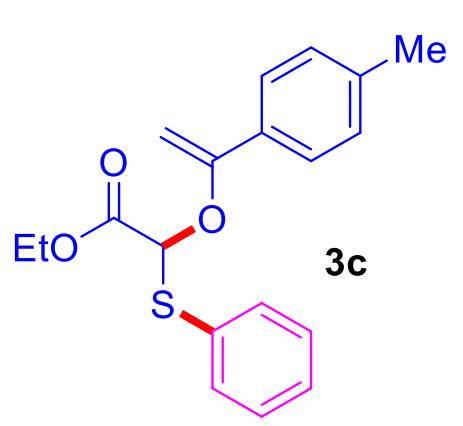

Following the general procedure, treatment of 2-(trimethylsilyl) phenyltrifluoromethanesulfonate $2 \mathrm{a}(0.179 \mathrm{~g}, 146 \mu \mathrm{L}, 0.6 \mathrm{mmol})$ with ethyl 2-((2-oxo-2-(p-tolyl)ethyl)thio)acetate 1c (0.119 g, 0.5 $\mathrm{mmol})$ in the presence of $\mathrm{KF}(0.070 \mathrm{~g}, 1.2 \mathrm{mmol})$ and 18-crown-6 $(0.317 \mathrm{~g}, 1.2 \mathrm{mmol})$ in THF $(2.0 \mathrm{~mL})$ at $25^{\circ} \mathrm{C}$ for $3 \mathrm{~h}$ followed by flash column chromatography (Pet.ether/EtOAc $=97 / 03)$ of the crude reaction mixture using silica gel afforded ethyl 2-(phenylthio)-

2-((1-(p-tolyl)vinyl)oxy)acetate $3 \mathbf{c}$ as a colorless oil ( $0.113 \mathrm{~g}, 69 \%$ yield).

$\boldsymbol{R}_{\mathbf{f}}\left(\right.$ Pet. ether /EtOAc = 95/05): 0.48; ${ }^{1} \mathbf{H}$ NMR (400 MHz, $\left.\mathbf{C D C l}_{3}\right) \delta$ 7.60-7.54 (m, 4H), 7.36-7.31 (m, 3H), $7.17(\mathrm{~d}, J=8.0 \mathrm{~Hz}, 2 \mathrm{H}), 5.73(\mathrm{~s}, 1 \mathrm{H}), 4.87$ (d, $J=3.5 \mathrm{~Hz}, 1 \mathrm{H}), 4.31(\mathrm{~d}, J=3.5 \mathrm{~Hz}, 1 \mathrm{H})$, 4.21-4.13 (m, 2H), $2.38(\mathrm{~s}, 3 \mathrm{H}), 1.20(\mathrm{t}, J=7.1 \mathrm{~Hz}, 3 \mathrm{H}) .{ }^{\mathbf{1 3}} \mathbf{C} \mathbf{~ N M R}\left(\mathbf{1 0 0} \mathbf{~ M H z}, \mathbf{C D C l}_{3}\right) \delta 166.9$, 158.0, 138.9, 134.5, 132.6, 130.8, 129.0, 125.7, 86.2, 81.2, 61.7, 21.3, 14.1. HRMS (ESI) m/z: $[\mathrm{M}+\mathrm{Na}]^{+}$Calcd for $\mathrm{C}_{19} \mathrm{H}_{20} \mathrm{NaO}_{3} \mathrm{~S}$ 351.1025; Found 351.1030. FTIR $\left(\mathbf{c m}^{-1}\right)$ 3055, 2928, 1738, $1603,1325,1028$.

\section{Ethyl-2-((1-(4-bromophenyl)vinyl)oxy)-2-(phenylthio)acetate (3d)}

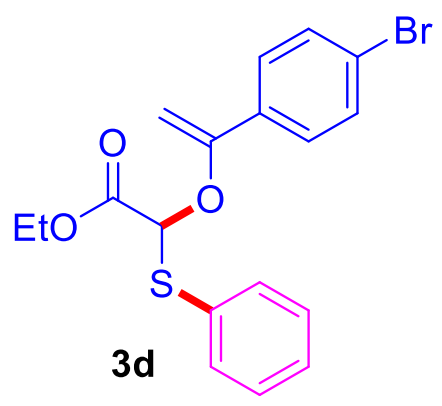

Following the general procedure, treatment of 2-(trimethylsilyl) phenyltrifluoromethanesulfonate $2 \mathbf{a}(0.179 \mathrm{~g}, 146 \mu \mathrm{L}, 0.6 \mathrm{mmol})$ with ethyl 2-((2-(4-bromophenyl)-2-oxoethyl)thio)acetate 1d (0.159 g, 0.5 $\mathrm{mmol})$ in the presence of $\mathrm{KF}(0.070 \mathrm{~g}, 1.2 \mathrm{mmol})$ and 18-crown-6 $(0.317 \mathrm{~g}, 1.2 \mathrm{mmol})$ in THF $(2.0 \mathrm{~mL})$ at $25^{\circ} \mathrm{C}$ for $3 \mathrm{~h}$ followed by flash column chromatography (Pet.ether/EtOAc $=97 / 03)$ of the crude reaction mixture using silica gel afforded ethyl-2-((1-(4bromophenyl)vinyl)oxy)-2-(phenylthio)acetate $3 \mathbf{d}$ as a colourless oil ( $0.175 \mathrm{~g}, 89 \%$ yield).

$\boldsymbol{R} \mathbf{f}($ Pet. ether $/$ EtOAc $=95 / 05): 0.41 ;{ }^{1} \mathbf{H}$ NMR $\left(\mathbf{4 0 0} \mathbf{~ M H z}, \mathbf{C D C l}_{3}\right) \delta$ 7.56-7.54 (m, 2H), 7.51-7.45 (m, 4H), 7.38-7.30 (m, 3H), $5.71(\mathrm{~s}, 1 \mathrm{H}), 4.89$ (d, $J=3.8 \mathrm{~Hz}, 1 \mathrm{H}), 4.35$ (d, $J=3.8 \mathrm{~Hz}, 1 \mathrm{H}), 4.22-$ 
$4.10(\mathrm{~m}, 2 \mathrm{H}), 1.20(\mathrm{t}, J=7.1 \mathrm{~Hz}, 3 \mathrm{H}) .{ }^{13} \mathbf{C} \mathbf{N M R}\left(\mathbf{1 0 0} \mathbf{M H z}, \mathbf{C D C l}_{3}\right) \delta$ 166.7, 156.9, 134.6, 134.3, 131.5, 130.5, 129.2, 129.1, 127.4, 123.1, 87.3, 81.2, 62.0, 14.1. HRMS (ESI) m/z: [M+Na $]^{+}$Calcd for $\mathrm{C}_{18} \mathrm{H}_{17} \mathrm{BrNaO}_{3} \mathrm{~S}$ 414.9974; Found 414.9979. FTIR (cm $\left.{ }^{-1}\right)$ 2982, 1751, 1620, 1484, 1268, 1230, $1180,1114$.

Ethyl-2-((1-(4-chlorophenyl)vinyl)oxy)-2-(phenylthio)acetate (3e)

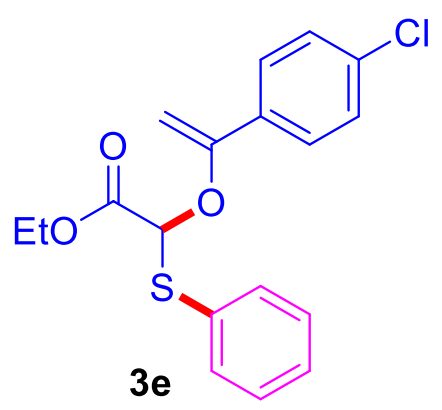

Following the general procedure, treatment of 2-(trimethylsilyl) phenyltrifluoromethanesulfonate $2 \mathbf{a}(0.179 \mathrm{~g}, 146 \mu \mathrm{L}, 0.6 \mathrm{mmol})$ with ethyl 2-((2-(4-chlorophenyl)-2-oxoethyl)thio)acetate 1e (0.136 g, 0.5 $\mathrm{mmol})$ in the presence of $\mathrm{KF}(0.070 \mathrm{~g}, 1.2 \mathrm{mmol})$ and 18-crown-6 $(0.317 \mathrm{~g}, 1.2 \mathrm{mmol})$ in THF $(2.0 \mathrm{~mL})$ at $25^{\circ} \mathrm{C}$ for $3 \mathrm{~h}$ followed by flash column chromatography (Pet.ether/EtOAc = 97/03) of the crude reaction mixture using silica gel afforded ethyl-2-((1-(4-

chlorophenyl)vinyl)oxy)-2-(phenylthio)acetate $\mathbf{3 e}$ as a colourless oil (0.152 g, 88\% yield).

$\boldsymbol{R}_{\mathbf{f}}($ Pet. ether $/$ EtOAc $=95 / 05)$ : 0.46; ${ }^{1} \mathbf{H}$ NMR (400 MHz, CDCl $) \delta$ 7.57-7.54 (m, 4H), 7.38-7.30 (m, 5H), $5.71(\mathrm{~s}, 1 \mathrm{H}), 4.88(\mathrm{~d}, J=3.8 \mathrm{~Hz}, 1 \mathrm{H}), 4.34(\mathrm{~d}, J=3.8 \mathrm{~Hz}, 1 \mathrm{H}), 4.22-4.10(\mathrm{~m}, 2 \mathrm{H}), 1.20$

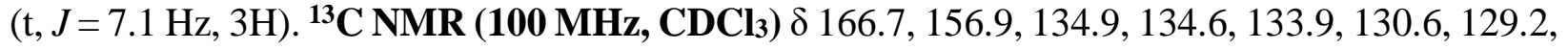
129.1, 128.5, 127.1, 87.2, 81.2, 62.0, 14.1. HRMS (ESI) m/z: $[\mathrm{M}+\mathrm{Na}]^{+}$Calcd for $\mathrm{C}_{18} \mathrm{H}_{17} \mathrm{ClNaO}_{3} \mathrm{~S}$ 371.0479; Found 371.0485. FTIR (cm-1) 2983, 1751, 1621, 1489, 1269, 1116, 1016, 833.

Ethyl-2-((1-(4-fluorophenyl)vinyl)oxy)-2-(phenylthio)acetate (3f)<smiles>C=C(OC(Sc1ccccc1)C(=O)OCC)c1ccc(F)cc1</smiles>

Following the general procedure, treatment of 2-(trimethylsilyl) phenyltrifluoromethanesulfonate $2 \mathbf{a}(0.179 \mathrm{~g}, 146 \mu \mathrm{L}, 0.6 \mathrm{mmol})$ with ethyl 2-((2-(4-fluorophenyl)-2-oxoethyl)thio)acetate $\mathbf{1 f}(0.128 \mathrm{~g}, 0.5$ $\mathrm{mmol})$ in the presence of $\mathrm{KF}(0.070 \mathrm{~g}, 1.2 \mathrm{mmol})$ and 18-crown-6 $(0.317 \mathrm{~g}, 1.2 \mathrm{mmol})$ in $\mathrm{THF}(2.0 \mathrm{~mL})$ at $25^{\circ} \mathrm{C}$ for $1.5 \mathrm{~h}$ followed by flash column chromatography (Pet.ether/EtOAc $=97 / 03)$ of the crude reaction mixture using silica gel afforded ethyl-2-((1-(4fluorophenyl)vinyl)oxy)-2-(phenylthio)acetate $3 \mathbf{f}$ as a pale yellow oil (0.145 g, 87\% yield).

$\boldsymbol{R}_{\mathbf{f}}($ Pet. ether $/$ EtOAc $=95 / 05)$ : 0.47; ${ }^{1} \mathbf{H}$ NMR (400 MHz, CDCl $) \delta$ 7.63-7.59 (m, 2H), 7.58-7.55 $(\mathrm{m}, 2 \mathrm{H}), 7.38-7.30(\mathrm{~m}, 3 \mathrm{H}), 7.06-7.00(\mathrm{~m}, 2 \mathrm{H}), 5.71(\mathrm{~s}, 1 \mathrm{H}), 4.83(\mathrm{~d}, J=3.8 \mathrm{~Hz}, 1 \mathrm{H}), 4.31(\mathrm{~d}, J=$ 
3.8 Hz, 1H), 4.22-4.11 (m, 2H), $1.20(\mathrm{t}, J=7.1 \mathrm{~Hz}, 3 \mathrm{H}) .{ }^{\mathbf{1 3}} \mathbf{C}$ NMR (100 MHz, CDCl $) \delta 166.8$, $163.3(\mathrm{~d}, J=248.9 \mathrm{~Hz}), 157.1,134.6,131.6(\mathrm{~d}, J=3.1 \mathrm{~Hz}), 130.6,129.2,129.1,127.7(\mathrm{~d}, J=8.4$ Hz), $115.2(\mathrm{~d}, J=21.7 \mathrm{~Hz}), 86.6,81.2,62.0,14.1$. HRMS (ESI) m/z: $[\mathrm{M}+\mathrm{Na}]^{+}$Calcd for $\mathrm{C}_{18} \mathrm{H}_{17} \mathrm{FNaO}_{3} \mathrm{~S}$ 355.0775; Found 355.0783. FTIR (cm-1) 3436, 2984, 1734, 1275, 1068, 1021, 859.

Ethyl-2-((1-(4-cyanophenyl)vinyl)oxy)-2-(phenylthio)acetate (3g)

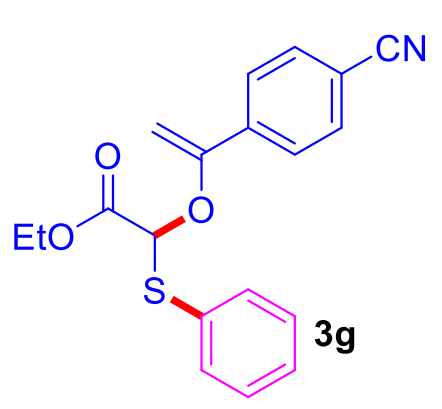

Following the general procedure, treatment of 2-(trimethylsilyl) phenyltrifluoromethanesulfonate $2 \mathbf{a}(0.179 \mathrm{~g}, 146 \mu \mathrm{L}, 0.6 \mathrm{mmol})$ with ethyl 2-((2-(4-cyanophenyl)-2-oxoethyl)thio)acetate $1 \mathrm{~g}(0.132 \mathrm{~g}, 0.5$ $\mathrm{mmol})$ in the presence of $\mathrm{KF}(0.070 \mathrm{~g}, 1.2 \mathrm{mmol})$ and 18-crown-6 $(0.317 \mathrm{~g}, 1.2 \mathrm{mmol})$ in THF $(2.0 \mathrm{~mL})$ at $25^{\circ} \mathrm{C}$ for $1 \mathrm{~h}$ followed by flash column chromatography (Pet.ether/EtOAc = 92/08) of the crude

reaction mixture using silica gel afforded ethyl-2-((1-(4-cyanophenyl)vinyl)oxy)-2(phenylthio)acetate $\mathbf{3 g}$ as a colorless oil ( $0.149 \mathrm{~g}, 88 \%$ yield).

$\boldsymbol{R}_{\mathbf{f}}\left(\right.$ Pet. ether $/ \mathrm{EtOAc}=$ 95/05): 0.46; ${ }^{1} \mathbf{H}$ NMR (400 MHz, CDCl 3$) \delta 7.70(\mathrm{~d}, J=8.7 \mathrm{~Hz}, 2 \mathrm{H})$, $7.61(\mathrm{~d}, J=8.7 \mathrm{~Hz}, 2 \mathrm{H}), 7.53-7.51(\mathrm{~m}, 2 \mathrm{H}), 7.38-7.29(\mathrm{~m}, 3 \mathrm{H}), 5.71(\mathrm{~s}, 1 \mathrm{H}), 5.00(\mathrm{~d}, J=4.3 \mathrm{~Hz}$, 1H), 4.47 (d, $J=4.1 \mathrm{~Hz}, 1 \mathrm{H}), 4.21-4.10(\mathrm{~m}, 2 \mathrm{H}), 1.19$ (t, $J=7.1 \mathrm{~Hz}, 3 \mathrm{H}) .{ }^{13} \mathbf{C}$ NMR (100 MHz, CDCl3) $\delta 166.4,155.9,139.5,134.6,132.1,130.2,129.3,129.1,126.2,118.7,112.4,89.5,81.2$, 62.1, 14.0. HRMS (ESI) m/z: [M+H] ${ }^{+}$Calcd for $\mathrm{C}_{19} \mathrm{H}_{18} \mathrm{NO}_{3} \mathrm{~S} 340.1002$; Found 340.1007. FTIR $\left(\mathbf{c m}^{-1}\right)$ 2985, 2228, 1751, 1617, 1441, 1115.

\section{Ethyl-2-((1-(4-nitrophenyl)vinyl)oxy)-2-(phenylthio)acetate (3h)}

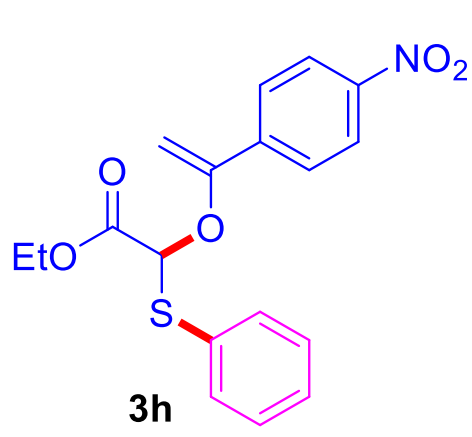

Following the general procedure, treatment of 2-(trimethylsilyl) phenyltrifluoromethanesulfonate $2 \mathbf{a}(0.179 \mathrm{~g}, 146 \mu \mathrm{L}, 0.6 \mathrm{mmol})$ with ethyl 2-((2-(4-nitrophenyl)-2-oxoethyl)thio)acetate $\mathbf{1 h}(0.142$ $\mathrm{g}, 0.5 \mathrm{mmol})$ in the presence of $\mathrm{KF}(0.070 \mathrm{~g}, 1.2 \mathrm{mmol})$ and 18 crown-6 (0.317 g, $1.2 \mathrm{mmol})$ in THF $(2.0 \mathrm{~mL})$ at $25{ }^{\circ} \mathrm{C}$ for $1 \mathrm{~h}$ followed by flash column chromatography (Pet.ether/EtOAc = 97/03) of the crude reaction mixture using silica gel afforded ethyl2-((1-(4-nitrophenyl)vinyl)oxy)-2-(phenylthio)acetate $\mathbf{3 h}$ as a colourless oil (0.127 g, $71 \%$ yield). 
$\boldsymbol{R}_{\mathbf{f}}\left(\right.$ Pet. ether $/$ EtOAc = 95/05): 0.41; ${ }^{1} \mathbf{H}$ NMR (400 MHz, $\left.\mathbf{C D C l}_{3}\right) \delta 8.19(\mathrm{~d}, J=9.0 \mathrm{~Hz}, 2 \mathrm{H}), 7.77$ $(\mathrm{d}, J=9.0 \mathrm{~Hz}, 2 \mathrm{H}), 7.55-7.52(\mathrm{~m}, 2 \mathrm{H}), 7.43-7.31(\mathrm{~m}, 3 \mathrm{H}), 5.72(\mathrm{~s}, 1 \mathrm{H}), 5.07(\mathrm{~d}, J=4.2 \mathrm{~Hz}, 1 \mathrm{H})$, $4.52(\mathrm{~d}, J=4.2 \mathrm{~Hz}, 1 \mathrm{H}), 4.23-4.12(\mathrm{~m}, 2 \mathrm{H}), 1.21(\mathrm{t}, J=7.1 \mathrm{~Hz}, 3 \mathrm{H}) .{ }^{13} \mathbf{C}$ NMR (100 MHz, CDCl$)$ $\delta 166.5,155.8,148.1,141.4,134.8,130.2,129.5,129.2,126.5,123.7,90.2,81.3,62.2,14.1$. HRMS (ESI) m/z: [M+Na] ${ }^{+}$Calcd for $\mathrm{C}_{18} \mathrm{H}_{17} \mathrm{NNaO}_{5} \mathrm{~S}$ 382.0720; Found 382.0726. FTIR (cm 1) $3058,2984,1752,1594,1520,1342,1234,1184,1111$.

\section{Ethyl-2-((1-(3-bromophenyl)vinyl)oxy)-2-(phenylthio)acetate (3i)}

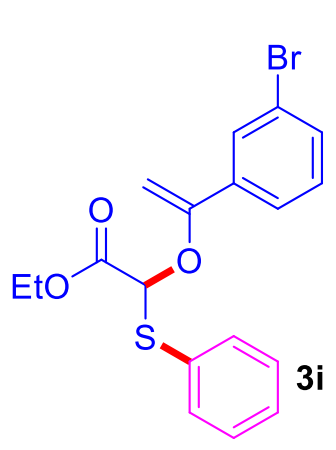

Following the general procedure, treatment of 2-(trimethylsilyl) phenyltrifluoromethanesulfonate $2 \mathbf{a}(0.179 \mathrm{~g}, 146 \mu \mathrm{L}, 0.6 \mathrm{mmol})$ with ethyl 2-((2-(3-bromophenyl)-2-oxoethyl)thio)acetate $1 \mathbf{i}(0.158 \mathrm{~g}, 0.5 \mathrm{mmol})$ in the presence of KF (0.070 g, $1.2 \mathrm{mmol})$ and 18-crown-6 (0.317 g, $1.2 \mathrm{mmol})$ in THF (2.0 mL) at $25^{\circ} \mathrm{C}$ for $3 \mathrm{~h}$ followed by flash column chromatography 3i (Pet.ether/EtOAc $=97 / 03)$ of the crude reaction mixture using silica gel afforded ethyl-2-((1-(3-bromophenyl)vinyl)oxy)-2-(phenylthio)acetate $\mathbf{3 i}$ as a colorless oil $(0.169 \mathrm{~g}, 86 \%$ yield).

$\boldsymbol{R}_{\mathbf{f}}\left(\right.$ Pet. ether /EtOAc = 95/05): 0.46; ${ }^{1} \mathbf{H}$ NMR (400 MHz, CDCl3) $\delta$ 7.77-7.76 (m, 1H), 7.58-7.54 $(\mathrm{m}, 3 \mathrm{H}), 7.47-7.45(\mathrm{~m}, 1 \mathrm{H}), 7.37-7.26(\mathrm{~m}, 3 \mathrm{H}), 7.21(\mathrm{t}, J=7.9 \mathrm{~Hz}, 1 \mathrm{H}) 5.71(\mathrm{~s}, 1 \mathrm{H}), 4.89(\mathrm{~d}, J=$ $3.9 \mathrm{~Hz}, 1 \mathrm{H}), 4.37(\mathrm{~d}, J=3.9 \mathrm{~Hz}, 1 \mathrm{H}), 4.22-4.11(\mathrm{~m}, 2 \mathrm{H}), 1.20(\mathrm{t}, J=7.1 \mathrm{~Hz}, 3 \mathrm{H}) .{ }^{13} \mathbf{C} \mathbf{N M R}(\mathbf{1 0 0}$ MHz, CDCl $) \delta 166.6,156.5,137.4,134.7,131.9,130.4,129.8,129.3,129.1,128.8,124.3,122.5$, 87.9, 81.2, 62.0, 14.1. HRMS (ESI) $\mathrm{m} / \mathrm{z}$ : $[\mathrm{M}+\mathrm{Na}]^{+}$Calcd for $\mathrm{C}_{18} \mathrm{H}_{17} \mathrm{BrNaO}_{3} \mathrm{~S}$ 414.9974; Found 414.9978. FTIR $\left(\mathbf{c m}^{-1}\right)$ 3064, 2983, 1752, 1622, 1475, 1265.

\section{Ethyl-2-((1-(3-chlorophenyl)vinyl)oxy)-2-(phenylthio)acetate (3j)}<smiles>C=C(OC(Sc1ccccc1)C(=O)OCC)c1cccc(Cl)c1</smiles>

Following the general procedure, treatment of 2-(trimethylsilyl) phenyltrifluoromethanesulfonate $2 \mathbf{a}(0.179 \mathrm{~g}, 146 \mu \mathrm{L}, 0.6 \mathrm{mmol})$ with ethyl 2-((2-(3-chlorophenyl)-2-oxoethyl)thio)acetate $\mathbf{1 j}(0.136 \mathrm{~g}, 0.5 \mathrm{mmol})$ in the presence of $\mathrm{KF}(0.070 \mathrm{~g}, 1.2 \mathrm{mmol})$ and 18 -crown-6 $(0.317 \mathrm{~g}, 1.2 \mathrm{mmol})$ in $\mathrm{THF}(2.0 \mathrm{~mL})$ at $25^{\circ} \mathrm{C}$ for $3 \mathrm{~h}$ followed by flash column chromatography

3j (Pet.ether/EtOAc $=97 / 03)$ of the crude reaction mixture using silica gel 
afforded ethyl-2-((1-(3-chlorophenyl)vinyl)oxy)-2-(phenylthio)acetate $\mathbf{3 j}$ as a colorless oil (0.146 g, $84 \%$ yield).

$\boldsymbol{R}_{\mathbf{f}}\left(\right.$ Pet. ether $/$ EtOAc = 95/05): 0.46; ${ }^{1} \mathbf{H}$ NMR (400 MHz, CDCl3) $\delta$ 7.61-7.56 (m, 3H), 7.51-7.49 (m, 1H), 7.39-7.25 (m, 5H), $5.71(\mathrm{~s}, 1 \mathrm{H}), 4.90(\mathrm{~d}, J=3.8 \mathrm{~Hz}, 1 \mathrm{H}), 4.37(\mathrm{~d}, J=3.9 \mathrm{~Hz}, 1 \mathrm{H}), 4.22-$ $4.11(\mathrm{~m}, 2 \mathrm{H}), 1.20(\mathrm{t}, J=7.1 \mathrm{~Hz}, 3 \mathrm{H}) .{ }^{13} \mathbf{C}$ NMR (100 MHz, CDCl3) $\delta$ 166.7, 156.6, 137.2, 134.7, 134.4 130.5, 129.6, 129.3, 129.1, 129.0, 126.0, 123.9, 87.8, 81.3, 62.0, 14.1. HRMS (ESI) m/z: $[\mathrm{M}+\mathrm{Na}]^{+}$Calcd for $\mathrm{C}_{18} \mathrm{H}_{17} \mathrm{ClNaO}_{3} \mathrm{~S}$ 371.0479; Found 371.0481. FTIR (cm-1) 3066, 2984, 2903, $1752,1566,1253$.

\section{Ethyl-2-((1-(3-nitrophenyl)vinyl)oxy)-2-(phenylthio)acetate (3k)}

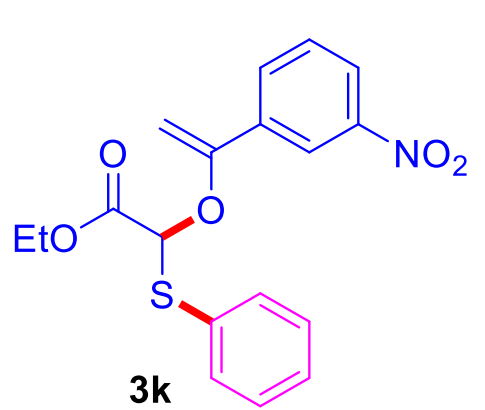

Following the general procedure, treatment of 2-(trimethylsilyl) phenyltrifluoromethanesulfonate $2 \mathbf{a}(0.179 \mathrm{~g}, 146 \mu \mathrm{L}, 0.6 \mathrm{mmol})$ with ethyl 2-((2-(3-nitrophenyl)-2-oxoethyl)thio)acetate $\mathbf{1 k}(0.142$ $\mathrm{g}, 0.5 \mathrm{mmol})$ in the presence of $\mathrm{KF}(0.070 \mathrm{~g}, 1.2 \mathrm{mmol})$ and 18 crown-6 (0.317 g, $1.2 \mathrm{mmol})$ in THF $(2.0 \mathrm{~mL})$ at $25{ }^{\circ} \mathrm{C}$ for $3 \mathrm{~h}$ followed by flash column chromatography (Pet.ether/EtOAc $=$

90/10) of the crude reaction mixture using silica gel afforded ethyl-2-((1-(3nitrophenyl)vinyl)oxy)-2-(phenylthio)acetate 3k as a pale yellow oil (0.149 g, $83 \%$ yield).

$\boldsymbol{R}_{\mathbf{f}}($ Pet. ether $/$ EtOAc $=90 / 10)$ : 0.31; ${ }^{1} \mathbf{H}$ NMR (400 MHz, CDCl3) $\delta$ 8.47-8.46 (m, 1H), 8.19-8.16 (m, 1H), $7.92(\mathrm{~d}, J=7.9 \mathrm{~Hz}, 1 \mathrm{H}), 7.58-7.56(\mathrm{~m}, 2 \mathrm{H}), 7.51(\mathrm{t}, J=8.0 \mathrm{~Hz}, 1 \mathrm{H}), 7.40-7.32(\mathrm{~m}, 3 \mathrm{H})$, $5.72(\mathrm{~s}, 1 \mathrm{H}), 5.01(\mathrm{~d}, J=4.2 \mathrm{~Hz}, 1 \mathrm{H}), 4.45(\mathrm{~d}, J=4.2 \mathrm{~Hz}, 1 \mathrm{H}), 4.22-4.10(\mathrm{~m}, 2 \mathrm{H}), 1.20(\mathrm{t}, J=7.1$

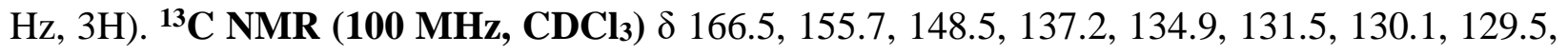
129.4, 129.2, 123.6, 120.8, 88.7, 81.3, 62.2, 14.1. HRMS (ESI) m/z: $[\mathrm{M}+\mathrm{H}]^{+}$Calcd for

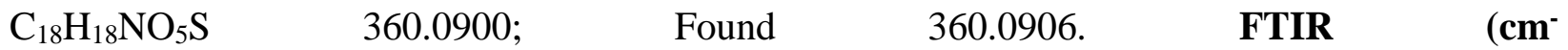
1) 2983, 1750, 1624, 1530, 1347, 1182, 1135, 1023.

\section{Ethyl-2-((1-(2-methoxyphenyl)vinyl)oxy)-2-(phenylthio)acetate (3I)}

Following the general procedure, treatment of 2-(trimethylsilyl) phenyltrifluoromethanesulfonate 2a $(0.179 \mathrm{~g}, 146 \mu \mathrm{L}, 0.6 \mathrm{mmol})$ with ethyl 2-((2-(2-methoxyphenyl)-2-oxoethyl)thio)acetate 11 $(0.134 \mathrm{~g}, 0.5 \mathrm{mmol})$ in the presence of $\mathrm{KF}(0.070 \mathrm{~g}, 1.2 \mathrm{mmol})$ and 18 -crown-6 (0.317 g, $1.2 \mathrm{mmol})$ in $\mathrm{THF}(2.0 \mathrm{~mL})$ at $25^{\circ} \mathrm{C}$ for $2 \mathrm{~h}$ followed by flash column chromatography (Pet.ether/EtOAc $=$ 
<smiles>C=C(OC(Sc1ccccc1)C(=O)OCC)c1ccccc1OC</smiles>

97/03) of the crude reaction mixture using silica gel afforded ethyl-2-((1(2-methoxyphenyl)vinyl)oxy)-2-(phenylthio)acetate 31 as a pale yellow oil ( $0.143 \mathrm{~g}, 83 \%$ yield).

$\boldsymbol{R}_{\mathbf{f}}(\mathrm{Pet}$. ether $/ \mathrm{EtOAc}=95 / 05): 0.46 ;{ }^{1} \mathbf{H}$ NMR $\left(\mathbf{4 0 0} \mathbf{~ M H z}, \mathbf{C D C l}_{\mathbf{3}}\right) \delta 7.59-$ $7.57(\mathrm{~m}, 2 \mathrm{H}), 7.51\left(\mathrm{dd}, J_{1}=7.6 \mathrm{~Hz}, J_{2}=1.6 \mathrm{~Hz}, 1 \mathrm{H}\right), 7.35-7.29(\mathrm{~m}, 4 \mathrm{H})$, 6.97-6.90 (m, 2H), 5.66 (s, 1H), 4.97 (d, $J=2.6 \mathrm{~Hz}, 1 \mathrm{H}), 4.65$ (d, $J=2.6$ $\mathrm{Hz}, 1 \mathrm{H}), 4.20-4.09$ (m, 2H), 3.83 (s, 3H), 1.18 (t, $J=7.1 \mathrm{~Hz}, 3 \mathrm{H}) .{ }^{13} \mathrm{C}$ NMR (100 MHz, CDCl $) \delta 167.0,157.2,155.4,134.3,131.2,130.04,130.0,129.0,128.8,124.5$, 120.5, 111.3, 93.0, 81.4, 61.8, 55.6, 14.1. HRMS (ESI) m/z: $[\mathrm{M}+\mathrm{Na}]^{+}$Calcd for $\mathrm{C}_{19} \mathrm{H}_{20} \mathrm{NaO}_{4} \mathrm{~S}$ : 367.0975, found: 367.0980. FTIR ( cm$\left.^{-1}\right)$ 3439, 2981, 1742, 1671, 1598, 1485, 1436, 1246, 1021.

\section{Ethyl-2-((1-(2-chlorophenyl)vinyl)oxy)-2-(phenylthio)acetate (3m)}

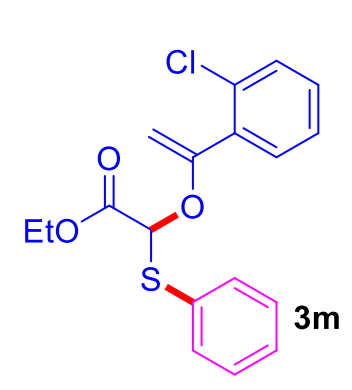

Following the general procedure, treatment of 2-(trimethylsilyl) phenyltrifluoromethanesulfonate $2 \mathrm{a}(0.179 \mathrm{~g}, 146 \mu \mathrm{L}, 0.6 \mathrm{mmol})$ with ethyl 2-((2-(2-chlorophenyl)-2-oxoethyl)thio)acetate $1 \mathrm{~m}(0.136 \mathrm{~g}, 0.5 \mathrm{mmol})$ in the presence of $\mathrm{KF}(0.070 \mathrm{~g}, 1.2 \mathrm{mmol})$ and 18-crown-6 (0.317 g, $1.2 \mathrm{mmol})$ in THF $(2.0 \mathrm{~mL})$ at $25^{\circ} \mathrm{C}$ for $3 \mathrm{~h}$ followed by flash column chromatography (Pet.ether/EtOAc $=97 / 03)$ of the crude reaction mixture using silica gel afforded ethyl-2-((1-(2-chlorophenyl)vinyl)oxy)-2-(phenylthio)acetate $\mathbf{3 m}$ as a colorless oil (0.141 g, 81\% yield).

$\boldsymbol{R}_{\mathbf{f}}\left(\right.$ Pet. ether /EtOAc = 95/05): 0.46; ${ }^{1} \mathbf{H}$ NMR (400 MHz, $\left.\mathbf{C D C l}_{\mathbf{3}}\right) \delta$ 7.59-7.57 (m, 2H), 7.44-7.37 (m, 2H), 7.34-7.21 (m, 5H), 5.64 (s, 1H), 4.66-4.64 (m, 2H), 4.22-4.10 (m, 2H), 1.19 (t, $J=7.1$ $\mathrm{Hz}, 3 \mathrm{H}) .{ }^{13} \mathbf{C}$ NMR (100 MHz, CDCl$) \delta$ 166.7, 156.4, 135.1, 134.5, 132.8, 131.2, 130.8, 130.0, 123.0, 129.0, 126.6, 93.0, 81.6, 61.9, 14.1. HRMS (ESI) m/z: [M+Na] ${ }^{+}$Calcd for $\mathrm{C}_{18} \mathrm{H}_{17} \mathrm{ClNaO}_{3} \mathrm{~S}$ 371.0479; Found 371.0486. FTIR ( cm$\left.^{-1}\right)$ 3061, 2983, 1756, 1633, 1475, 1298.

\section{Ethyl-2-((1-(2-nitrophenyl)vinyl)oxy)-2-(phenylthio)acetate (3n)}

Following the general procedure, treatment of 2-(trimethylsilyl) phenyltrifluoromethanesulfonate 2a $(0.090 \mathrm{~g}, 73 \mu \mathrm{L}, 0.3 \mathrm{mmol})$ with ethyl 2-((2-(2-nitrophenyl)-2-oxoethyl)thio)acetate 1n (0.071 $\mathrm{g}, 0.25 \mathrm{mmol})$ in the presence of $\mathrm{KF}(0.035 \mathrm{~g}, 0.6 \mathrm{mmol})$ and 18 -crown-6 $(0.158 \mathrm{~g}, 0.6 \mathrm{mmol})$ in THF $(1.0 \mathrm{~mL})$ at $25{ }^{\circ} \mathrm{C}$ for $2 \mathrm{~h}$ followed by flash column chromatography (Pet.ether/EtOAc $=$ 


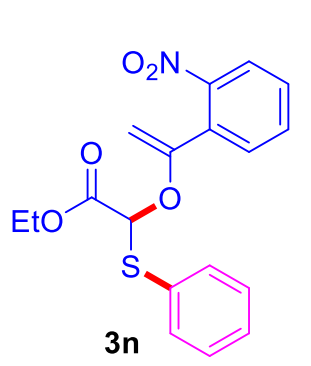

90/10) of the crude reaction mixture using silica gel afforded ethyl-2-((1-(2nitrophenyl)vinyl)oxy)-2-(phenylthio)acetate $3 \mathbf{n}$ as a pale yellow oil (0.076 g, $84 \%$ yield).

$\boldsymbol{R}_{\mathbf{f}}($ Pet. ether $/ \mathrm{EtOAc}=90 / 10): 0.31 ;{ }^{1} \mathbf{H}$ NMR $\left(\mathbf{4 0 0} \mathbf{~ M H z}, \mathbf{C D C l}_{3}\right) \delta 7.82(\mathrm{~d}$, $J=7.9 \mathrm{~Hz}, 1 \mathrm{H}), 7.59-7.47$ (m, 5H), 7.35-7.27 (m, 3H), 5.64 (s, 1H), 4.05 (d, $J$ $=3.8 \mathrm{~Hz}, 1 \mathrm{H}), 4.53(\mathrm{~d}, J=3.8 \mathrm{~Hz}, 1 \mathrm{H}), 4.18-4.06(\mathrm{~m}, 2 \mathrm{H}), 1.17(\mathrm{t}, J=7.1 \mathrm{~Hz}$,

3H). ${ }^{13}$ C NMR (100 MHz, CDCl $) \delta 166.3,156.7,148.9,134.8,132.6,131.3,131.2,130.4,130.0$, 129.2, 129.1, 124.3, 91.0, 82.8, 62.1, 14.0. HRMS (ESI) m/z: [M+Na] ${ }^{+}$Calcd for $\mathrm{C}_{18} \mathrm{H}_{17} \mathrm{NNaO}_{5} \mathrm{~S}$ 382.0720; Found: 382.0722. FTIR ( cm$\left.^{-1}\right)$ 3061, 1752, 1636, 1531, 1362, 1127, 1052

\section{Ethyl-2-((1-(3,4-dimethoxyphenyl)vinyl)oxy)-2-(phenylthio)acetate (3o)}

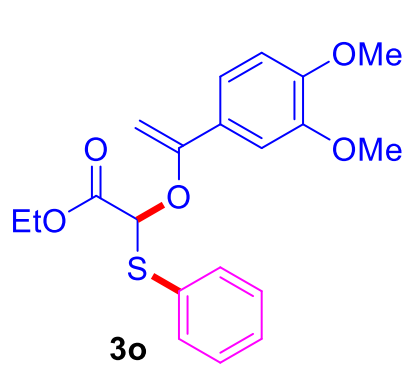

Following the general procedure, treatment of 2-(trimethylsilyl) phenyltrifluoromethanesulfonate $2 \mathrm{a}(0.179 \mathrm{~g}, 146 \mu \mathrm{L}, 0.6 \mathrm{mmol})$ with ethyl 2-((2-(3,4-dimethoxyphenyl)-2-oxoethyl)thio)acetate $10(0.149 \mathrm{~g}$, $0.5 \mathrm{mmol})$ in the presence of $\mathrm{KF}(0.070 \mathrm{~g}, 1.2 \mathrm{mmol})$ and 18 -crown- 6 $(0.317 \mathrm{~g}, 1.2 \mathrm{mmol})$ in THF $(2.0 \mathrm{~mL})$ at $25^{\circ} \mathrm{C}$ for $2.5 \mathrm{~h}$ followed by flash column chromatography $($ Pet.ether/EtOAc $=97 / 03)$ of the crude reaction mixture using silica gel afforded ethyl-2-((1-(3,4-dimethoxyphenyl)vinyl)oxy)-2(phenylthio)acetate 30 as a colourless oil $(0.151 \mathrm{~g}, 81 \%$ yield).

$\boldsymbol{R} \mathbf{f}($ Pet. ether /EtOAc $=95 / 05): 0.31 ;{ }^{1} \mathbf{H}$ NMR $\left(\mathbf{4 0 0} \mathbf{~ M H z}, \mathbf{C D C l}_{3}\right) \delta$ 7.57-7.55 (m, 2H), 7.36-7.28 $(\mathrm{m}, 3 \mathrm{H}), 7.21\left(\mathrm{dd}, J_{1}=8.4 \mathrm{~Hz}, J_{2}=1.9 \mathrm{~Hz}, 1 \mathrm{H}\right), 7.14(\mathrm{~d}, J=1.9 \mathrm{~Hz}, 1 \mathrm{H}), 5.71(\mathrm{~s}, 1 \mathrm{H}), 4.79(\mathrm{~d}, J$ $=3.6 \mathrm{~Hz}, 1 \mathrm{H}), 4.26(\mathrm{~d}, J=3.6 \mathrm{~Hz}, 1 \mathrm{H}), 4.19-4.11(\mathrm{~m}, 2 \mathrm{H}), 3.89(\mathrm{~s}, 3 \mathrm{H}), 3.86(\mathrm{~s}, 3 \mathrm{H}), 1.18(\mathrm{t}, J=$ $7.1 \mathrm{~Hz}, 3 \mathrm{H}) .{ }^{13} \mathbf{C} \mathbf{N M R}\left(\mathbf{1 0 0} \mathbf{~ M H z}, \mathbf{C D C l}_{3}\right) \delta 167.0,157.8,149.8,148.7,134.6,130.8,129.1$, 129.0, 128.4, 118.7, 110.9, 109.1, 85.84, 81.1, 61.9, 56.1, 55.9, 14.1. HRMS (ESI) m/z: [M+Na] ${ }^{+}$ Calcd for $\mathrm{C}_{20} \mathrm{H}_{22} \mathrm{NaO}_{5} \mathrm{~S}$ 397.1080; Found 397.1082. FTIR ( cm$\left.^{-1}\right)$ 2936, 1738, 1671, 1589, 1541, 1270, 1021.

\section{Ethyl-2-((1-(3-bromo-4-fluorophenyl)vinyl)oxy)-2-(phenylthio)acetate (3p)}

Following the general procedure, treatment of 2-(trimethylsilyl) phenyltrifluoromethanesulfonate 2a $(0.179 \mathrm{~g}, 146 \mu \mathrm{L}, 0.6 \mathrm{mmol})$ with ethyl ethyl 2-((2-(3-bromo-4-fluorophenyl)-2oxoethyl)thio)acetate $1 \mathbf{p}(0.168 \mathrm{~g}, 0.5 \mathrm{mmol})$ in the presence of $\mathrm{KF}(0.070 \mathrm{~g}, 1.2 \mathrm{mmol})$ and 18 - 


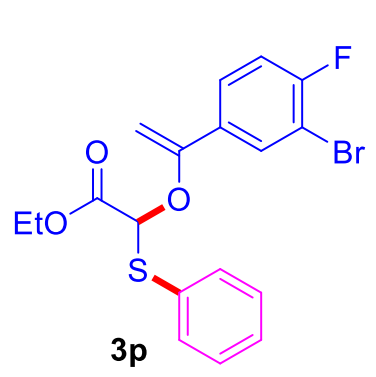

crown-6 $(0.317 \mathrm{~g}, 1.2 \mathrm{mmol})$ in THF $(2.0 \mathrm{~mL})$ at $25{ }^{\circ} \mathrm{C}$ for $2 \mathrm{~h}$ followed by flash column chromatography (Pet.ether/EtOAc $=97 / 03)$ of the crude reaction mixture using silica gel afforded Ethyl-2-((1-(3-bromo-4fluorophenyl)vinyl)oxy)-2-(phenylthio)acetate $3 p$ as a pale yellow oil (0.162 g, 79\% yield).

$\boldsymbol{R}_{\mathbf{f}}\left(\right.$ Pet. ether $/ \mathrm{EtOAc}=$ 95/05): 0.49; ${ }^{1} \mathbf{H}$ NMR $\left(\mathbf{4 0 0} \mathbf{~ M H z}, \mathbf{C D C l}_{3}\right) \delta 7.79$ $\left(\mathrm{dd}, J_{l}=6.6 \mathrm{~Hz}, J_{2}=2.2 \mathrm{~Hz}, 1 \mathrm{H}\right), 7.56-7.50(\mathrm{~m}, 3 \mathrm{H}), 7.40-7.32(\mathrm{~m}, 3 \mathrm{H}), 7.07(\mathrm{t}, J=8.4 \mathrm{~Hz}, 1 \mathrm{H})$, $5.68(\mathrm{~s}, 1 \mathrm{H}), 4.82(\mathrm{~d}, J=4.0 \mathrm{~Hz}, 1 \mathrm{H}), 4.33(\mathrm{~d}, J=4.0 \mathrm{~Hz}, 1 \mathrm{H}), 4.22-4.11(\mathrm{~m}, 2 \mathrm{H}), 1.20(\mathrm{t}, J=7.1$ $\mathrm{Hz}, 3 \mathrm{H}) .{ }^{13} \mathbf{C}$ NMR $\left(\mathbf{1 0 0} \mathbf{M H z}, \mathbf{C D C l}_{3}\right) \delta 166.7,159.5(\mathrm{~d}, J=249.5 \mathrm{~Hz}), 155.9,134.8,133.1(\mathrm{~d}, J$ $=3.8 \mathrm{~Hz}), 131.1,130.4,129.4,129.2,126.5(\mathrm{~d}, J=7.4 \mathrm{~Hz}), 116.3(\mathrm{~d}, J=22.6 \mathrm{~Hz}), 109.1(\mathrm{~d}, J=$ $21.3 \mathrm{~Hz}$ ), 87.6, 81.3, 62.1, 14.1. HRMS (ESI) m/z: $[\mathrm{M}+\mathrm{Na}]^{+}$Calcd for $\mathrm{C}_{18} \mathrm{H}_{16} \mathrm{BrFNaO}_{3} \mathrm{~S}$ 432.9880; Found 432.9886. FTIR (cm-1) 2983, 1750, 1623, 1494, 1264, 1181, 1058, 953.

\section{Ethyl-2-((1-(naphthalen-2-yl)vinyl)oxy)-2-(phenylthio)acetate (3q)}

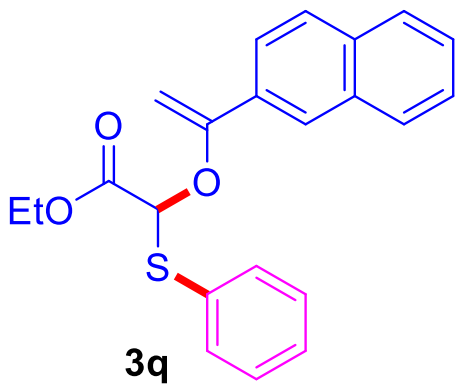

Following the general procedure, treatment of 2-(trimethylsilyl) phenyltrifluoromethanesulfonate 2a $(0.179 \mathrm{~g}, 146 \mu \mathrm{L}, 0.6 \mathrm{mmol})$ with ethyl 2-((2-(naphthalen-2-yl)-2-oxoethyl)thio)acetate 1q $(0.144 \mathrm{~g}, 0.5 \mathrm{mmol})$ in the presence of $\mathrm{KF}(0.070 \mathrm{~g}, 1.2 \mathrm{mmol})$ and 18-crown-6 (0.317 g, $1.2 \mathrm{mmol})$ in THF $(2.0 \mathrm{~mL})$ at $25^{\circ} \mathrm{C}$ for $3 \mathrm{~h}$ followed by flash column chromatography (Pet.ether/EtOAc $=$ 97/03) of the crude reaction mixture using silica gel afforded ethyl-2-((1-(naphthalen-2yl)vinyl)oxy)-2-(phenylthio)acetate $3 q$ as a pale yellow oil ( $0.135 \mathrm{~g}, 74 \%$ yield).

$\boldsymbol{R}_{\mathbf{f}}($ Pet. ether /EtOAc $=95 / 05): 0.46 ;{ }^{1} \mathbf{H}$ NMR $\left(400 \mathbf{~ M H z}, \mathbf{C D C l}_{3}\right) \delta 8.14(\mathrm{~s}, 1 \mathrm{H}), 7.88-7.80(\mathrm{~m}$, $3 \mathrm{H}), 7.71\left(\mathrm{dd}, J_{1}=8.6 \mathrm{~Hz}, \mathrm{~J}_{2}=1.7 \mathrm{~Hz}, 1 \mathrm{H}\right), 7.63-7.61(\mathrm{~m}, 2 \mathrm{H}), 7.52-7.47(\mathrm{~m}, 2 \mathrm{H}), 7.40-7.32(\mathrm{~m}$, 3H), $5.80(\mathrm{~s}, 1 \mathrm{H}), 5.06(\mathrm{~d}, J=3.7 \mathrm{~Hz}, 1 \mathrm{H}), 4.46(\mathrm{~d}, J=3.7 \mathrm{~Hz}, 1 \mathrm{H}), 4.26-4.14(\mathrm{~m}, 2 \mathrm{H}), 1.23(\mathrm{t}, J$ $=7.1 \mathrm{~Hz}, 3 \mathrm{H}) .{ }^{13} \mathbf{C} \mathbf{~ N M R}\left(\mathbf{1 0 0} \mathbf{~ M H z}, \mathbf{C D C l}_{3}\right) \delta 167.0,157.9,134.7,133.7,133.2,132.6,130.7$, 129.2, 129.1, 128.7, 127.9, 127.7, 126.6, 126.4, 125.2, 123.6, 87.7, 81.3, 62.0, 14.1. HRMS (ESI) m/z: $\quad[\mathrm{M}+\mathrm{Na}]^{+}$Calcd for $\mathrm{C}_{22} \mathrm{H}_{20} \mathrm{NaO}_{3} \mathrm{~S}$ 387.1025; Found 387.1029. FTIR (cm1) $3438,3058,2982,1749,1678,1626,1472,1284$. 


\section{Ethyl-2-((1-(naphthalen-1-yl)vinyl)oxy)-2-(phenylthio)acetate (3r)}

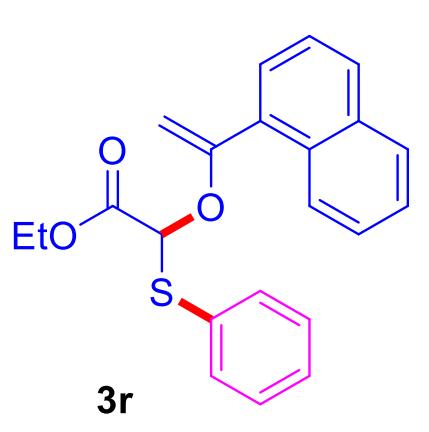

Following the general procedure, treatment of 3(trimethylsilyl)naphthalen-2-yl trifluoromethanesulfonate $\mathbf{2 a}(0.179 \mathrm{~g}$, $146 \mu \mathrm{L}, \quad 0.6 \mathrm{mmol}) \quad$ with ethyl 2-((2-(naphthalen-1-yl)-2oxoethyl)thio)acetate $1 \mathbf{r}(0.144 \mathrm{~g}, 0.5 \mathrm{mmol})$ in the presence of $\mathrm{KF}$ $(0.070 \mathrm{~g}, 1.2 \mathrm{mmol})$ and 18-crown-6 (0.317 g, $1.2 \mathrm{mmol})$ in THF (2.0 $\mathrm{mL})$ at $25^{\circ} \mathrm{C}$ for $3 \mathrm{~h}$ followed by flash column chromatography (Pet.ether/EtOAc $=97 / 03)$ of the crude reaction mixture using silica gel afforded ethyl-2-((1-(naphthalen-1-yl)vinyl)oxy)-2-(phenylthio)acetate $\mathbf{3 r}$ as a colourless oil (0.165 g, 91\% yield).

$\boldsymbol{R}_{\mathbf{f}}($ Pet. ether $/$ EtOAc $=95 / 05): 0.48 ;{ }^{1} \mathbf{H}$ NMR (400 MHz, CDCl 3$) \delta 8.32(\mathrm{~d}, J=7.8 \mathrm{~Hz}, 1 \mathrm{H})$, 7.89-7.87 (m, 2H), 7.59-7.56 (m, 3H), 7.53-7.44 (m, 3H), 7.39- 7.30 (m, 3H), 5.69 (s, 1H), 4.70 $(\mathrm{d}, J=2.8 \mathrm{~Hz}, 1 \mathrm{H}), 4.67(\mathrm{~d}, J=2.8 \mathrm{~Hz}, 1 \mathrm{H}), 4.25-4.11(\mathrm{~m}, 2 \mathrm{H}), 1.21(\mathrm{t}, J=7.1 \mathrm{~Hz}, 3 \mathrm{H}) .{ }^{13} \mathbf{C ~ N M R}$ (100 MHz, CDCl 3$) \delta 166.9,159.1,134.7,134.0,133.6,131.3,130.7,129.6,129.1,129.0,128.2$, 127.6, 126.4, 126.1, 126.0, 125.1, 92.6, 81.4, 62.0, 14.1. HRMS (ESI) m/z: [M+Na] ${ }^{+}$Calcd for $\mathrm{C}_{22} \mathrm{H}_{20} \mathrm{NaO}_{3} \mathrm{~S}$ 387.1025: Found 387.1030. FTIR (cm-1) 3052, 2927, 1748, 1623, 1439, 1250, 1192, 1067.

\section{Ethyl-2-((1-(3,5-difluorophenyl)vinyl)oxy)-2-(phenylthio)acetate (3s)}

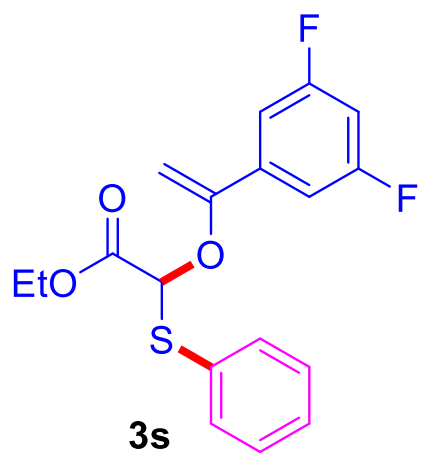

Following the general procedure, treatment of 2-(trimethylsilyl) phenyltrifluoromethanesulfonate $2 \mathbf{a}(0.179 \mathrm{~g}, 146 \mu \mathrm{L}, 0.6 \mathrm{mmol})$ with ethyl 2-((2-(3,5-difluorophenyl)-2-oxoethyl)thio)acetate 1s $(0.137 \mathrm{~g}$, $0.5 \mathrm{mmol})$ in the presence of $\mathrm{KF}(0.070 \mathrm{~g}, 1.2 \mathrm{mmol})$ and 18-crown$6(0.317 \mathrm{~g}, 1.2 \mathrm{mmol})$ in THF $(2.0 \mathrm{~mL})$ at $25{ }^{\circ} \mathrm{C}$ for $2 \mathrm{~h}$ followed by flash column chromatography (Pet.ether/EtOAc $=97 / 03)$ of the crude reaction mixture using silica gel afforded ethyl-2-((1-(3,5difluorophenyl)vinyl)oxy)-2-(phenylthio)acetate 3s as a colourless oil (0.141 g, 81\% yield).

$\boldsymbol{R}_{\mathbf{f}}\left(\right.$ Pet. ether $/ \mathrm{EtOAc}=$ 95/05): 0.46; ${ }^{1} \mathbf{H}$ NMR (400 MHz, CDCl $) \delta$ 7.56-7.54 (m, 2H), 7.40-7.31 $(\mathrm{m}, 3 \mathrm{H}), 7.16-7.10(\mathrm{~m}, 2 \mathrm{H}), 6.78\left(\mathrm{tt}, J_{1}=8.7 \mathrm{~Hz}, J_{2}=2.3 \mathrm{~Hz}, 1 \mathrm{H}\right), 5.68(\mathrm{~s}, 1 \mathrm{H}), 4.91(\mathrm{~d}, J=4.1$ $\mathrm{Hz}, 1 \mathrm{H}), 4.39(\mathrm{~d}, J=4.1 \mathrm{~Hz}, 1 \mathrm{H}), 4.22-4.10(\mathrm{~m}, 2 \mathrm{H}), 1.20(\mathrm{t}, J=7.1 \mathrm{~Hz} 3 \mathrm{H}) .{ }^{\mathbf{1 3}} \mathbf{C} \mathbf{N M R}(\mathbf{1 0 0} \mathbf{M H z}$, $\left.\mathbf{C D C l}_{3}\right) \delta 166.6,163.1(\mathrm{~d}, J=247.41 \mathrm{~Hz}), 163.0(\mathrm{~d}, J=247.41 \mathrm{~Hz}), 155.7(\mathrm{t}, J=3.2 \mathrm{~Hz}), 138.8$ 
(t, $J=9.8 \mathrm{~Hz}), 134.8,130.3,129.4,129.2,108.9(\mathrm{~d}, J=7.47 \mathrm{~Hz}), 108.7(\mathrm{~d}, J=7.47 \mathrm{~Hz}), 104.3(\mathrm{t}$, $J=25.5 \mathrm{~Hz}$ ), 88.6, 81.3, 62.1, 14.1. HRMS (ESI) m/z: $[\mathrm{M}+\mathrm{Na}]^{+}$Calcd for $\mathrm{C}_{18} \mathrm{H}_{16} \mathrm{~F}_{2} \mathrm{NaO}_{3} \mathrm{~S}$ 373.0680; Found 373.0684. FTIR (cm $\left.\mathbf{c m}^{-1}\right)$ 2984, 1753, 1624, 1589, 1342, 1118, 987.

\section{Ethyl-2-(phenylthio)-2-((1-(thiophen-2-yl)vinyl)oxy)acetate (3t)}

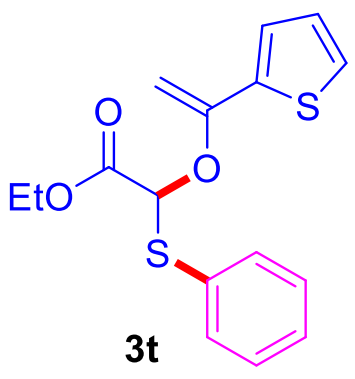

Following the general procedure, treatment of 2-(trimethylsilyl) phenyltrifluoromethanesulfonate $2 \mathrm{a}(0.179 \mathrm{~g}, 146 \mu \mathrm{L}, 0.6 \mathrm{mmol})$ with ethyl 2-((2-oxo-2-(thiophen-2-yl)ethyl)thio)acetate $1 \mathbf{t}(0.122 \mathrm{~g}, 0.5 \mathrm{mmol})$ in the presence of $\mathrm{KF}(0.070 \mathrm{~g}, 1.2 \mathrm{mmol})$ and 18-crown-6 (0.317 g, 1.2 $\mathrm{mmol})$ in THF $(2.0 \mathrm{~mL})$ at $25{ }^{\circ} \mathrm{C}$ for $3 \mathrm{~h}$ followed by flash column chromatography (Pet.ether/EtOAc $=97 / 03)$ of the crude reaction mixture

using silica gel afforded ethyl-2-(phenylthio)-2-((1-(thiophen-2-yl)vinyl)oxy)acetate $\mathbf{3 t}$ as a colourless oil ( $0.116 \mathrm{~g}, 73 \%$ yield).

$\boldsymbol{R}_{\mathbf{f}}\left(\right.$ Pet. ether /EtOAc $=$ 95/05): 0.46; ${ }^{\mathbf{1}} \mathbf{H}$ NMR $\left(\mathbf{4 0 0} \mathbf{~ M H z}, \mathbf{C D C l}_{\mathbf{3}}\right) \delta$ 7.60-7.58 (m, 2H), 7.38-7.33 (m, 3H), 7.30-7.29 (m, 1H), 7.25 (d, $J=5.3 \mathrm{~Hz}, 1 \mathrm{H}), 7.01-6.99$ (m, 1H), 5.70 (s, 1H), 4.84 (d, $J$ $=3.9 \mathrm{~Hz}, 1 \mathrm{H}), 4.25(\mathrm{~d}, J=3.9 \mathrm{~Hz}, 1 \mathrm{H}), 4.20-4.08(\mathrm{~m}, 2 \mathrm{H}), 1.18(\mathrm{t}, J=7.1 \mathrm{~Hz}, 3 \mathrm{H}) .{ }^{13} \mathbf{C} \mathbf{~ N M R}$ (100 MHz, CDCl3) $\delta 166.6,153.2,139.2,134.7,130.5,129.2,129.1,127.4,125.8,124.9,86.0$, 81.3, 62.0, 14.1. HRMS (ESI) m/z: [M+Na] ${ }^{+}$Calcd for $\mathrm{C}_{16} \mathrm{H}_{16} \mathrm{NaO}_{3} \mathrm{~S}_{2} 343.0433$; Found 343.0438. FTIR $\left(\mathbf{c m}^{-1}\right)$ 3436, 2983, 2363, 1734, 1660, 1413, 1276, 1068, 1021.

\section{Methyl-2-(phenylthio)-2-((1-phenylvinyl)oxy)acetate (3u)}

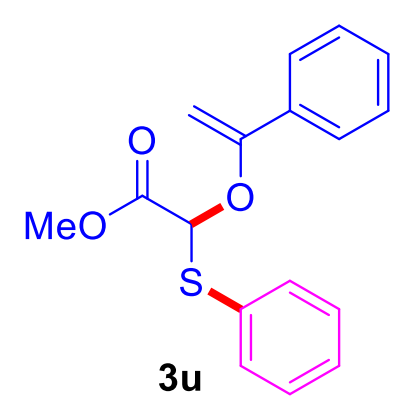

Following the general procedure, treatment of 2-(trimethylsilyl) phenyltrifluoromethanesulfonate $2 \mathrm{a}(0.179 \mathrm{~g}, 146 \mu \mathrm{L}, 0.6 \mathrm{mmol})$ with methyl 2-((2-oxo-2-phenylethyl)thio)acetate $1 \mathbf{u}(0.112 \mathrm{~g}, 0.5 \mathrm{mmol})$ in the presence of $\mathrm{KF}(0.070 \mathrm{~g}, 1.2 \mathrm{mmol})$ and 18-crown-6 $(0.317 \mathrm{~g}, 1.2$ $\mathrm{mmol})$ in THF $(2.0 \mathrm{~mL})$ at $25{ }^{\circ} \mathrm{C}$ for $3 \mathrm{~h}$ followed by flash column chromatography (Pet.ether/EtOAc $=97 / 03)$ of the crude reaction mixture using silica gel afforded methyl-2-(phenylthio)-2-((1-phenylvinyl)oxy)acetate $3 \mathbf{u}$ as a colourless oil $(0.135 \mathrm{~g}, 90 \%$ yield $)$.

$\boldsymbol{R}_{\mathbf{f}}\left(\mathrm{Pet}\right.$. ether /EtOAc = 95/05): 0.49; ${ }^{\mathbf{1}} \mathbf{H}$ NMR $\left(\mathbf{4 0 0} \mathbf{~ M H z}, \mathbf{C D C l}_{\mathbf{3}}\right) \delta$ 7.66-7.64 (m, 2H), 7.58-7.57 (m, 2H), 7.39-7.31 (m, 6H), $5.77(\mathrm{~s}, 1 \mathrm{H}), 4.92(\mathrm{~d}, J=3.7 \mathrm{~Hz}, 1 \mathrm{H}), 4.34(\mathrm{~d}, J=3.7 \mathrm{~Hz}, 1 \mathrm{H}), 3.71$ 
(s, 3H). ${ }^{13}$ C NMR (100 MHz, $\left.\mathbf{C D C l}_{3}\right) \delta$ 167.3, 157.9, 135.3, 134.6, 130.7, 129.2, 129.1, 129.0, 128.3, 125.8, 87.0, 81.3, 52.7. HRMS (ESI) $\mathrm{m} / \mathrm{z}:[\mathrm{M}+\mathrm{Na}]^{+}$Calcd for $\mathrm{C}_{17} \mathrm{H}_{16} \mathrm{NaO}_{3} \mathrm{~S}$ 323.0712; Found 323.0717. FTIR $\left(\mathbf{c m}^{-1}\right)$ 3436, 2954, 1740, 1679, 1440, 1274, 1166, 1069.

\section{Butyl-2-(phenylthio)-2-((1-phenylvinyl)oxy)acetate (3v)}

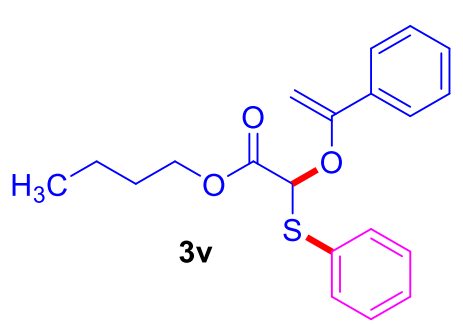

Following the general procedure, treatment of 2-(trimethylsilyl) phenyltrifluoromethanesulfonate 2a $(0.179 \mathrm{~g}, 146 \mu \mathrm{L}, 0.6 \mathrm{mmol})$ with butyl 2-((2-oxo-2-phenylethyl)thio)acetate 1v (0.133 g, 0.5 $\mathrm{mmol})$ in the presence of $\mathrm{KF}(0.070 \mathrm{~g}, 1.2 \mathrm{mmol})$ and 18-crown-6 $(0.317 \mathrm{~g}, 1.2 \mathrm{mmol})$ in THF $(2.0 \mathrm{~mL})$ at $25^{\circ} \mathrm{C}$ for $0.5 \mathrm{~h}$ followed by flash column chromatography (Pet.ether/EtOAc $=97 / 03)$ of the crude reaction mixture using silica gel afforded butyl-2-(phenylthio)-2-((1-phenylvinyl)oxy)acetate $\mathbf{3 v}$ as a colourless oil (0.149 g, $87 \%$ yield).

$\boldsymbol{R}_{\mathbf{f}}($ Pet. ether $/ \mathrm{EtOAc}=95 / 05): 0.43 ;{ }^{1} \mathbf{H}$ NMR $\left(\mathbf{4 0 0} \mathbf{~ M H z}, \mathbf{C D C l}_{3}\right) \delta$ 7.66-7.62 (m, 2H), 7.59-7.55 $(\mathrm{m}, 2 \mathrm{H}), 7.38-7.30(\mathrm{~m}, 6 \mathrm{H}), 5.73(\mathrm{~s}, 1 \mathrm{H}), 4.90(\mathrm{~d}, J=3.7 \mathrm{~Hz}, 1 \mathrm{H}), 4.33(\mathrm{~d}, J=3.7 \mathrm{~Hz}, 1 \mathrm{H}), 4.16-$ $4.04(\mathrm{~m}, 2 \mathrm{H}), 1.58-1.51(\mathrm{~m}, 2 \mathrm{H}), 1.37-1.28(\mathrm{~m}, 2 \mathrm{H}), 0.90(\mathrm{t}, J=7.4 \mathrm{~Hz}, 3 \mathrm{H}) .{ }^{13} \mathbf{C} \mathbf{~ N M R}(\mathbf{1 0 0} \mathbf{M H z}$, CDCl3) $\delta$ 167.0, 157.9, 135.4, 134.5, 130.8, 129.1, 129.08, 129.0, 128.3, 125.8, 86.9, 81.3, 65.8, 30.5, 19.1, 13.7. HRMS (ESI) m/z: [M+Na] ${ }^{+}$Calcd for $\mathrm{C}_{20} \mathrm{H}_{22} \mathrm{NaO}_{3} \mathrm{~S} 365.1182$; Found 365.1187. FTIR $\left(\mathbf{c m}^{-1}\right)$ 3059, 2961, 1755, 1733, 1620, 1441, 1276, 1177, 1118.

\section{Isopropyl-2-(phenylthio)-2-((1-phenylvinyl)oxy)acetate (3w)}

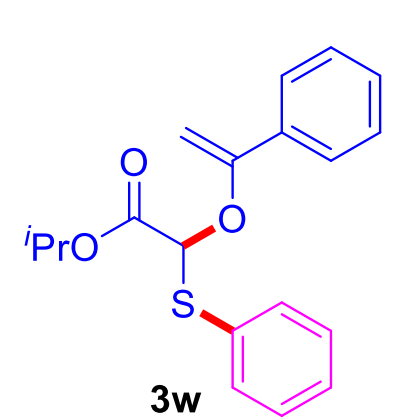

Following the general procedure, treatment of 2-(trimethylsilyl) phenyltrifluoromethanesulfonate $2 \mathrm{a}(0.179 \mathrm{~g}, 146 \mu \mathrm{L}, 0.6 \mathrm{mmol})$ with isopropyl 2-((2-oxo-2-phenylethyl)thio)acetate $1 \mathbf{w}(0.126 \mathrm{~g}, 0.5 \mathrm{mmol})$ in the presence of KF (0.070 g, $1.2 \mathrm{mmol})$ and 18-crown-6 (0.317 g, 1.2 $\mathrm{mmol})$ in THF $(2.0 \mathrm{~mL})$ at $25{ }^{\circ} \mathrm{C}$ for $3 \mathrm{~h}$ followed by flash column chromatography (Pet.ether/EtOAc $=97 / 03)$ of the crude reaction mixture using silica gel afforded isopropyl-2-(phenylthio)-2-((1-phenylvinyl)oxy)acetate $\mathbf{3 w}$ as a colourless oil ( $0.144 \mathrm{~g}, 88 \%$ yield).

$\boldsymbol{R} \mathbf{f}($ Pet. ether $/$ EtOAc $=95 / 05): 0.51 ;{ }^{1} \mathbf{H}$ NMR $\left(\mathbf{4 0 0} \mathbf{~ M H z}, \mathbf{C D C l}_{3}\right) \delta$ 7.68-7.65 (m, 2H), 7.61-7.59 (m, 2H), 7.39-7.30 (m, 6H), $5.71(\mathrm{~s}, 1 \mathrm{H}), 5.02(\mathrm{sep}, J=6.3 \mathrm{~Hz}, 1 \mathrm{H}), 4.90(\mathrm{~d}, J=3.6 \mathrm{~Hz}, 1 \mathrm{H}), 4.35$ 
$(\mathrm{d}, J=3.6 \mathrm{~Hz}, 1 \mathrm{H}), 1.21(\mathrm{~d}, J=6.3 \mathrm{~Hz}, 3 \mathrm{H}), 1.18(\mathrm{~d}, J=6.3 \mathrm{~Hz}, 3 \mathrm{H}) .{ }^{13} \mathrm{C}$ NMR (100 MHz, CDCl3) $\delta 166.3,158.1,135.5,134.4,130.9,129.0,128.95,128.3,125.8,86.8,81.3,69.8,21.6$, 21.6. HRMS (ESI) m/z: [M+Na] ${ }^{+}$Calcd for $\mathrm{C}_{19} \mathrm{H}_{20} \mathrm{NaO}_{3} \mathrm{~S}: 351.1025$, found: 351.1027. FTIR $\left(\mathbf{c m}^{-1}\right)$ 3439, 2982, 1728, 1281, 1103, 1067.

\section{Allyl-2-(phenylthio)-2-((1-phenylvinyl)oxy)acetate (3x)}

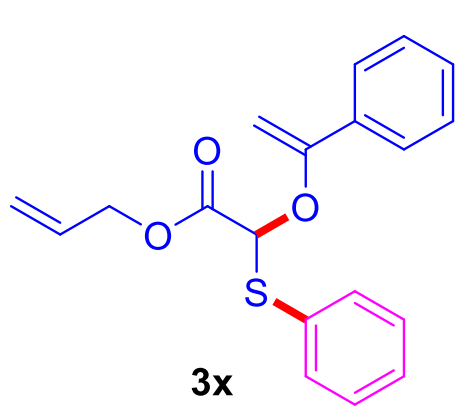

Following the general procedure, treatment of 2-(trimethylsilyl) phenyltrifluoromethanesulfonate $2 \mathbf{a}(0.179 \mathrm{~g}, 146 \mu \mathrm{L}, 0.6 \mathrm{mmol})$ with allyl 2-((2-oxo-2-phenylethyl)thio)acetate $\mathbf{1 x}(0.125 \mathrm{~g}, 0.5$ $\mathrm{mmol})$ in the presence of $\mathrm{KF}(0.070 \mathrm{~g}, 1.2 \mathrm{mmol})$ and 18-crown-6 $(0.317 \mathrm{~g}, 1.2 \mathrm{mmol})$ in THF $(2.0 \mathrm{~mL})$ at $25^{\circ} \mathrm{C}$ for $1.5 \mathrm{~h}$ followed by flash column chromatography (Pet.ether/EtOAc $=97 / 03)$ of the crude reaction mixture using silica gel afforded allyl-2-(phenylthio)-2-((1phenylvinyl)oxy)acetate $3 \mathbf{x}$ as a colourless oil ( $0.137 \mathrm{~g}, 84 \%$ yield).

$\boldsymbol{R}_{\mathbf{f}}($ Pet. ether $/$ EtOAc $=95 / 05)$ : 0.38; ${ }^{1} \mathbf{H}$ NMR (400 MHz, CDCl $) \delta$ 7.65-7.62 (m, 2H), 7.58-7.56 $(\mathrm{m}, 2 \mathrm{H}), 7.38-7.30(\mathrm{~m}, 6 \mathrm{H}), 5.83\left(\mathrm{ddt}, J_{1}=16.3 \mathrm{~Hz}, J_{2}=10.7 \mathrm{~Hz}, J_{3}=5.8 \mathrm{~Hz}, 1 \mathrm{H}\right), 5.76(\mathrm{~s}, 1 \mathrm{H})$, $5.32\left(\mathrm{dd}, J_{1}=17.3 \mathrm{~Hz}, J_{2}=1.3 \mathrm{~Hz}, 1 \mathrm{H}\right), 5.24\left(\mathrm{dd}, J_{1}=10.4 \mathrm{~Hz}, J_{2}=1.0 \mathrm{~Hz}, 1 \mathrm{H}\right), 4.92(\mathrm{~d}, J=3.7$ Hz, 1H), 4.66-4.54 (m, 2H), 4.35 (d, $J=3.7$ Hz, 1H) ${ }^{\mathbf{1 3}} \mathbf{C}$ NMR (100 MHz, CDCl $) \delta$ 166.6, 157.9, 135.3, 134.7, 131.4, 130.6, 129.2, 129.1, 129.0, 128.4, 125.8, 119.1, 87.0, 81.2, 66.3. HRMS (ESI) m/z; $[\mathrm{M}+\mathrm{Na}]+{ }^{+}$Calcd for $\mathrm{C}_{19} \mathrm{H}_{18} \mathrm{NaO}_{3} \mathrm{~S}$ 349.0869; Found: 349.0878. FTIR (cm $\left.{ }^{-1}\right)$ 3467, 2944, 1741, 1680, 1444, 1273, 1178, 933.

\section{Prop-2-yn-1-yl-2-(phenylthio)-2-((1-phenylvinyl)oxy)acetate (3y)}

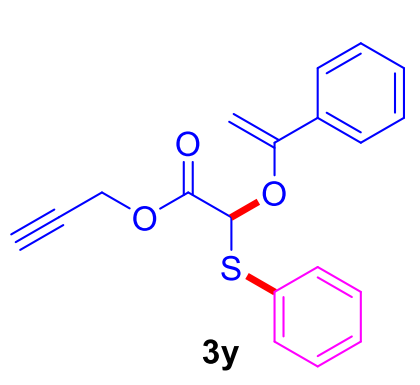

reaction mixture using silica gel afforded prop-2-yn-1-yl-2-(phenylthio)-2-((1-

Following the general procedure, treatment of 2-(trimethylsilyl) phenyltrifluoromethanesulfonate $2 \mathbf{a}(0.179 \mathrm{~g}, 146 \mu \mathrm{L}, 0.6 \mathrm{mmol})$ with prop-2-yn-1-yl 2-((2-oxo-2-phenylethyl)thio)acetate $1 \mathbf{y}(0.124 \mathrm{~g}, 0.5$ $\mathrm{mmol})$ in the presence of $\mathrm{KF}(0.070 \mathrm{~g}, 1.2 \mathrm{mmol})$ and 18-crown-6 $(0.317 \mathrm{~g}, 1.2 \mathrm{mmol})$ in THF $(2.0 \mathrm{~mL})$ at $25^{\circ} \mathrm{C}$ for $2 \mathrm{~h}$ followed by flash column chromatography (Pet.ether/EtOAc $=97 / 03$ ) of the crude phenylvinyl)oxy)acetate $\mathbf{3 y}$ as a colourless oil (0.113 g, $70 \%$ yield). 
$\boldsymbol{R}_{\mathbf{f}}($ Pet. ether $/$ EtOAc $=95 / 05): 0.39 ;{ }^{1} \mathbf{H}$ NMR (400 MHz, CDCl $\left.\mathbf{3}\right) \delta$ 7.64-7.61 (m, 2H), 7.59-7.57 $(\mathrm{m}, 2 \mathrm{H}), 7.39-7.31(\mathrm{~m}, 6 \mathrm{H}), 5.76(\mathrm{~s}, 1 \mathrm{H}), 4.93(\mathrm{~d}, J=3.7 \mathrm{~Hz}, 1 \mathrm{H}), 4.75-4.66(\mathrm{~m}, 2 \mathrm{H}), 4.35(\mathrm{~d}, J=$ $3.8 \mathrm{~Hz}, 1 \mathrm{H}), 2.51$ (t, $J=2.3 \mathrm{~Hz}, 1 \mathrm{H}) .{ }^{13} \mathrm{C}$ NMR (100 MHz, CDCl3) $\delta$ 166.1, 157.8, 135.2, 135.0, 130.2, 129.4, 129.2, 129.1, 128.4, 125.8, 87.2, 80.7, 75.8, 53.1. HRMS (ESI) m/z: [M+Na $]^{+}$Calcd for $\mathrm{C}_{19} \mathrm{H}_{16} \mathrm{NaO}_{3} \mathrm{~S}$ 347.0712; Found 347.0718. FTIR (cm-1) 3292, 2363, 1761, 1621, 1276, 1163 , 1118.

\section{Phenethyl-2-(phenylthio)-2-((1-phenylvinyl)oxy)acetate (3z)}

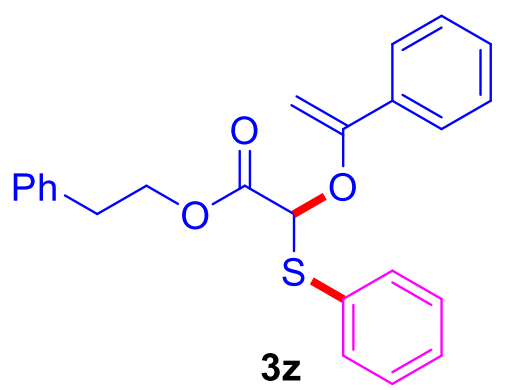

Following the general procedure, treatment of 2-(trimethylsilyl) phenyltrifluoromethanesulfonate $\mathbf{2 a}(0.179 \mathrm{~g}, 146 \mu \mathrm{L}, 0.6 \mathrm{mmol})$ with phenethyl 2-((2-oxo-2-phenylethyl)thio)acetate $\mathbf{1 z}(0.157 \mathrm{~g}$, $0.5 \mathrm{mmol})$ in the presence of $\mathrm{KF}(0.070 \mathrm{~g}, 1.2 \mathrm{mmol})$ and 18 crown-6 (0.317 g, $1.2 \mathrm{mmol})$ in THF $(2.0 \mathrm{~mL})$ at $25{ }^{\circ} \mathrm{C}$ for $1 \mathrm{~h}$ followed by flash column chromatography (Pet.ether/EtOAc $=$ 97/03) of the crude reaction mixture using silica gel afforded phenethyl-2-(phenylthio)-2-((1phenylvinyl)oxy)acetate $\mathbf{3 z}$ as a colourless oil (0.173 g, 89\% yield).

$\boldsymbol{R} \mathbf{f}($ Pet. ether $/$ EtOAc $=95 / 05)$ : 0.31; ${ }^{1} \mathbf{H}$ NMR (400 MHz, CDCl3) $\delta$ 7.69-7.66 (m, 2H), 7.57-7.55 (m, 2H), 7.43-7.25 (m, 9H), 7.24-7.22 (m, 2H), $5.77(\mathrm{~s}, 1 \mathrm{H}), 4.92(\mathrm{~d}, J=3.7 \mathrm{~Hz}, 1 \mathrm{H}), 4.40-4.27$ (m, 3H), 2.94-2.83 (m, 2H) ${ }^{13} \mathbf{C}$ NMR (100 MHz, CDCl3) $\delta$ 166.8, 157.7, 137.4, 135.3, 134.6, 130.6, 129.1, 129.03, 129.01, 129.0, 128.6, 128.3, 126.7, 125.7, 86.8, 81.1, 66.2, 34.9. HRMS (ESI) m/z: $[\mathrm{M}+\mathrm{Na}]^{+}$Calcd for $\mathrm{C}_{24} \mathrm{H}_{22} \mathrm{NaO}_{3} \mathrm{~S}$ 413.1182; Found 413.1187. FTIR (cm-1) 3059, $1754,1732,1620,1276,1117$.

\section{Phenyl-2-(phenylthio)-2-((1-phenylvinyl)oxy)acetate (3aa)}<smiles>C=C(OC(Sc1ccccc1)C(=O)O)c1ccccc1</smiles>

Following the general procedure, treatment of 2-(trimethylsilyl) phenyltrifluoromethanesulfonate $2 \mathrm{a}(0.179 \mathrm{~g}, 146 \mu \mathrm{L}, 0.6 \mathrm{mmol})$ with phenyl 2-((2-oxo-2-phenylethyl)thio)acetate 1aa $(0.143 \mathrm{~g}, 0.5 \mathrm{mmol})$ in the presence of KF (0.070 g, $1.2 \mathrm{mmol})$ and 18-crown-6 (0.317 g, $1.2 \mathrm{mmol})$ in THF (2.0 mL) at $25{ }^{\circ} \mathrm{C}$ for $0.5 \mathrm{~h}$ followed by flash column chromatography (Pet.ether/EtOAc $=97 / 03)$ of the crude reaction mixture using silica gel 
afforded phenyl-2-(phenylthio)-2-((1-phenylvinyl)oxy)acetate 3aa as a colourless oil (0.093 g, $51 \%$ yield).

$\boldsymbol{R}_{\mathbf{f}}($ Pet. ether $/$ EtOAc $=95 / 05): 0.35 ;{ }^{1} \mathbf{H}$ NMR (400 MHz, CDCl 3$) \delta$ 7.72-7.67 (m, 4H), 7.43-7.33 $(\mathrm{m}, 8 \mathrm{H}), 7.25-7.21(\mathrm{~m}, 1 \mathrm{H}), 6.95-6.93(\mathrm{~m}, 2 \mathrm{H}), 5.95(\mathrm{~s}, 1 \mathrm{H}), 5.01(\mathrm{~d}, J=3.7 \mathrm{~Hz}, 1 \mathrm{H}), 4.49(\mathrm{~d}, J=$ 3.7 Hz, 1H). ${ }^{13} \mathbf{C}$ NMR (100 MHz, CDCl3) $\delta$ 165.4, 158.0, 150.4, 135.3, 135.0, 130.3, 129.6, 129.4, 129.2, 129.1, 128.4, 126.3, 125.9, 121.3, 87.1, 81.0. HRMS (ESI) m/z: [M+Na ${ }^{+}$Calcd for $\mathrm{C}_{22} \mathrm{H}_{18} \mathrm{NaO}_{3} \mathrm{~S}$ 385.0869; Found 385.0870. FTIR (cm-1) 2852, 1772, 1591, 1489, 1276, 1191, $1116,1054$.

\section{$(1 R, 2 S, 5 R)-2-$ Isopropyl-5-methylcyclohexyl-2-(phenylthio)-2-((1-phenylvinyl)oxy)acetate} (3ab)

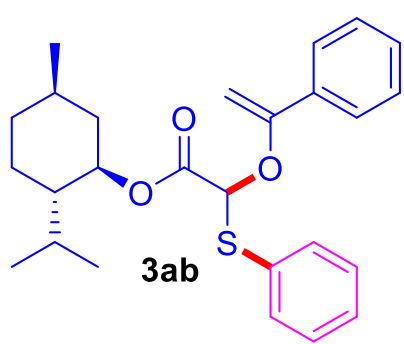

Following the general procedure, treatment of 2-(trimethylsilyl) phenyltrifluoromethanesulfonate $2 \mathbf{a}(0.179 \mathrm{~g}, 146 \mu \mathrm{L}, 0.6 \mathrm{mmol})$ with $(1 R, 2 S, 5 R)$-2-isopropyl-5-methylcyclohexyl 2-((2-oxo-2phenylethyl)thio)acetate $\mathbf{1 a b}(0.174 \mathrm{~g}, 0.5 \mathrm{mmol})$ in the presence of $\mathrm{KF}$ (0.070 g, $1.2 \mathrm{mmol})$ and 18-crown-6 (0.317 g, $1.2 \mathrm{mmol})$ in THF (2.0 $\mathrm{mL})$ at $25{ }^{\circ} \mathrm{C}$ for $1 \mathrm{~h}$ followed by flash column chromatography $($ Pet.ether/EtOAc $=97 / 03)$ of the crude reaction mixture using silica gel afforded $(1 R, 2 S, 5 R)-2$ Isopropyl-5-methylcyclohexyl-2-(phenylthio)-2-((1-phenylvinyl)oxy)acetate (4a) $\mathbf{3 a b}$ as a colourless oil (0.168 g, 79\% yield; diastereoselectivity determined by crude ${ }^{1} \mathrm{H}$ NMR is $\left.1: 1\right)$. $\boldsymbol{R}_{\mathbf{f}}($ Pet. ether $/$ EtOAc $=95 / 05): 0.51 ;{ }^{1} \mathbf{H}$ NMR of one Diastereomer $\left(400 \mathrm{MHz}, \mathbf{C D C l}_{3}\right) \delta 7.66-$ $7.62(\mathrm{~m}, 2 \mathrm{H}), 7.60-7.56(\mathrm{~m}, 2 \mathrm{H}), 7.38-7.29(\mathrm{~m}, 6 \mathrm{H}), 5.69$ (s, 1H), 4.87 (d, J = 3.7 Hz, 1H), 4.764.65 (m, 1H), 4.33-4.31 (m, 1H), 1.98-1.80 (m, 2H), 1.70-1.63 (m, 2H), 1.50-1.33 (m, 2H), 1.09$0.88(\mathrm{~m}, 7 \mathrm{H}), 0.82-0.81(\mathrm{~m}, 2 \mathrm{H}), 0.70(\mathrm{~d}, J=6.9 \mathrm{~Hz}, 3 \mathrm{H}) .{ }^{\mathbf{1}} \mathbf{H}$ NMR of other Diastereomer (400 MHz, CDCl $) \delta$ 7.66-7.62 (m, 2H), 7.60-7.56 (m, 2H), 7.38-7.29 (m, 6H), $5.70(\mathrm{~s}, 1 \mathrm{H}), 4.88(\mathrm{~d}, J$ $=3.6 \mathrm{~Hz}, 1 \mathrm{H}), 4.76-4.65(\mathrm{~m}, 1 \mathrm{H}), 4.33-4.31(\mathrm{~m}, 1 \mathrm{H}), 1.98-1.80(\mathrm{~m}, 2 \mathrm{H}), 1.70-1.63(\mathrm{~m}, 2 \mathrm{H}), 1.50-$ $1.33(\mathrm{~m}, 2 \mathrm{H}), 1.09-0.88(\mathrm{~m}, 7 \mathrm{H}), 0.82-0.81(\mathrm{~m}, 2 \mathrm{H}), 0.74(\mathrm{~d}, J=6.9 \mathrm{~Hz}, 3 \mathrm{H}) .{ }^{13} \mathrm{C}$ NMR of one Diastereomer (100 MHz, CDCl$) \delta$ 166.6, 158.3, 135.5, 134.0, 131.3, 129.1, 129.0, 128.8, 128.3, 125.8, 86.6, 81.6, 76.3, 47.0, 40.5, 31.5, 25.7, 23.1, 22.1, 20.9, $16.0{ }^{13} \mathbf{C}$ NMR of other Diastereomer (100 MHz, CDCl 3$) \delta 166.5,158.25,135.53,133.9,131.4,129.08,129.0,128.82$, 128.33, 125.9, 86.9, 81.55, 76.4, 46.9, 40.50, 31.2, 26.2, 23.4, 22.09, 20.90, 16.2 HRMS (ESI) 
m/z: $[\mathrm{M}+\mathrm{Na}]^{+}$Calcd for $\mathrm{C}_{26} \mathrm{H}_{32} \mathrm{NaO}_{3} \mathrm{~S}:$ 447.1964, found: 447.1970. FTIR (cm-1) 3429, 3377, 2952, 2861, 1727, 1447, 1185, 1078.

\section{(E)-3,7-Dimethylocta-2,6-dien-1-yl-2-(phenylthio)-2-((1-phenylvinyl)oxy)acetate (3ac)}

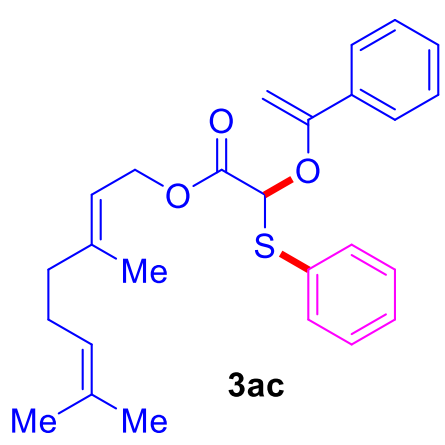

Following the general procedure, treatment of 2-(trimethylsilyl) phenyltrifluoromethanesulfonate $2 \mathbf{a}(0.179 \mathrm{~g}, 146 \mu \mathrm{L}, 0.6 \mathrm{mmol})$ with (E)-3,7-dimethylocta-2,6-dien-1-yl 2-((2-oxo-2-phenylethyl) thio)acetate 1 ac $(0.173 \mathrm{~g}, 0.5 \mathrm{mmol})$ in the presence of $\mathrm{KF}(0.070 \mathrm{~g}$, $1.2 \mathrm{mmol})$ and 18 -crown-6 (0.317 g, $1.2 \mathrm{mmol})$ in THF $(2.0 \mathrm{~mL})$ at $25{ }^{\circ} \mathrm{C}$ for $0.5 \mathrm{~h}$ followed by flash column chromatography $($ Pet.ether/EtOAc $=97 / 03)$ of the crude reaction mixture using silica gel afforded (E)-3,7-dimethylocta-2,6-dien-1-yl-2-(phenylthio)-2-((1-phenylvinyl)oxy)acetate 3ac as a colourless oil $(0.177 \mathrm{~g}, 84 \%$ yield $)$.

$\boldsymbol{R}_{\mathbf{f}}\left(\right.$ Pet. ether $/$ EtOAc = 95/05): 0.46; ${ }^{1} \mathbf{H}$ NMR (400 MHz, $\left.\mathbf{C D C l}_{3}\right) \delta$ 7.66-7.62 (m, 2H), 7.58-7.55 $(\mathrm{m}, 2 \mathrm{H}), 7.37-7.29(\mathrm{~m}, 6 \mathrm{H}), 5.73(\mathrm{~s}, 1 \mathrm{H}), 5.30-5.26(\mathrm{~m}, 1 \mathrm{H}), 5.12-5.08(\mathrm{~m}, 1 \mathrm{H}), 4.90(\mathrm{~d}, J=3.6$ $\mathrm{Hz}, 1 \mathrm{H}), 4.68-4.59$ (m, 2H), 4.33 (d, $J=3.6 \mathrm{~Hz}, 1 \mathrm{H}), 2.13-2.02$ (m, 4H), 1.70 (s, 3H), 1.69 (s, 3H), 1.62 (s, 3H). ${ }^{13} \mathbf{C}$ NMR (100 MHz, CDCl3) $\delta 166.8,157.9,143.4,135.4,134.6,132.0,130.8$, 129.1, 129.0, 128.3, 125.8, 123.8, 117.6, 87.0, 81.2, 62.7, 39.6, 26.4, 25.8, 17.8, 16.6. HRMS (ESI) $\mathrm{m} / \mathrm{z}: \quad[\mathrm{M}+\mathrm{Na}]^{+}$Calcd for $\mathrm{C}_{26} \mathrm{H}_{30} \mathrm{NaO}_{3} \mathrm{~S}$ 445.1808; Found 445.1813. FTIR (cm 1) $2921,1754,1620,1442,1276,1166,1118$.

(3S,8S,9S,10R,13R,14S,17R)-10,13-Dimethyl-17-((R)-6-methylheptan-2-yl) 2,3,4,7,8,9,10,11,12,13,14,15,16,17-tetradecahydro-1H-cyclopenta[a]phenanthren-3-yl 2 (phenylthio)-2-((1-phenylvinyl)oxy)acetate (3ad)

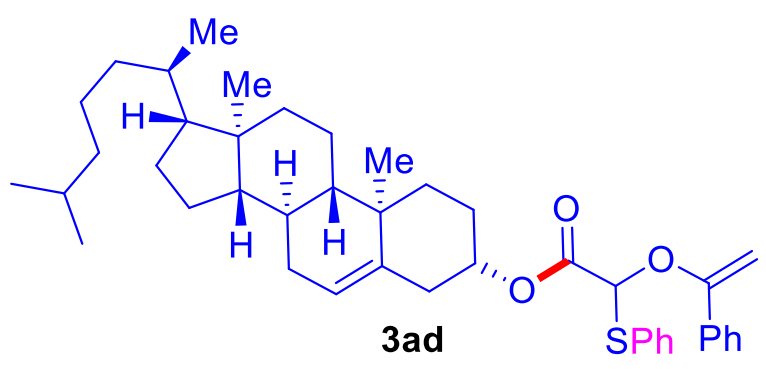

Following the general procedure, treatment of 2(trimethylsilyl) phenyltrifluoromethanesulfonate 2a $(0.179 \mathrm{~g}, \quad 146 \mu \mathrm{L}, \quad 0.6 \mathrm{mmol})$ with (3S,8S,9S,10R,13R,14S,17R)-10,13-dimethyl17-((R)-6-methylheptan-2-yl)$2,3,4,7,8,9,10,11,12,13,14,15,16,17-$

tetradecahydro-1H-cyclopenta[ $a]$ phenanthren-3-yl 2-((2-oxo-2-phenylethyl)thio)acetate $\mathbf{1 a d}$ $(0.289 \mathrm{~g}, 0.5 \mathrm{mmol})$ in the presence of $\mathrm{KF}(0.070 \mathrm{~g}, 1.2 \mathrm{mmol})$ and 18 -crown-6 (0.317 g, $1.2 \mathrm{mmol})$ 
in THF $(2.0 \mathrm{~mL})$ at $25^{\circ} \mathrm{C}$ for $2 \mathrm{~h}$ followed by flash column chromatography (Pet.ether/EtOAc $=$ 97/03) of the crude reaction mixture using silica gel afforded $(3 S, 8 S, 9 S, 10 R, 13 R, 14 S, 17 R)-10,13-$ dimethyl-17-((R)-6-methylheptan-2-yl) 2,3,4,7,8,9,10,11,12,13,14,15,16,17-tetradecahydro-1Hcyclopenta $[a]$ phenanthren-3-yl 2 (phenylthio)-2-((1-phenylvinyl)oxy)acetate 3ad as a colourless oil $\left(0.262 \mathrm{~g}, 80 \%\right.$ yield) (diastereomeric ratio determined by crude ${ }^{1} \mathrm{H}$ NMR in DMSO $\mathrm{d}_{6}$ is 1:1. For both diastereomers all peaks in ${ }^{1} \mathrm{H} \mathrm{nmr}\left(\mathrm{CDCl}_{3}\right)$ was merging, therefor combined peaks for both diastereomers are given).

$\boldsymbol{R}_{\mathbf{f}}\left(\right.$ Pet. ether /EtOAc = 95/05): 0.48; ${ }^{1} \mathbf{H}$ NMR (400 MHz, $\left.\mathbf{C D C l}_{3}\right) \delta$ 7.67-7.65 (m, 4H), 7.61-7.59 (m, 4H), 7.39-7.31 (m, 12H), $5.71(\mathrm{~s}, 2 \mathrm{H}), 5.36(\mathrm{~s}, 2 \mathrm{H}), 4.90(\mathrm{~d}, J=3.6 \mathrm{~Hz}, 2 \mathrm{H}), 4.67-4.58(\mathrm{~m}$, 2H), 4.35 (d, $J=3.6 \mathrm{~Hz}, 2 \mathrm{H}), 2.34-2.17(\mathrm{~m}, 4 \mathrm{H}), 2.05-1.97$ (m, 4H), 1.91-1.71 (m, 6H), 1.63-1.34 (m, 20H), 1.32-0.99 (m, 28H), 0.96-0.94 (m, 6H), 0.91-0.89 (m, 12H), 0.70 (s, 6H). ${ }^{13} \mathbf{C}$ NMR of one Diastereomer (100 MHz, CDCl3) $\delta$ 166.2, 158.1, 139.4, 135.5, 134.6, 130.9, 129.0, 128.96, $128.3,125.8,123.1,86.9,81.3,75.8,56.8,56.3,50.1,42.4,39.8,39.6,37.9,37.0,36.6,36.3,35.9$, $32.0,31.99,28.3,28.1,27.6,24.4,24.0,23.0,22.7,21.1,19.4,18.8,12.0 .{ }^{13} \mathbf{C}$ of other Diastereomer NMR (100 MHz, $\left.\mathbf{C D C l}_{3}\right) \delta$ 166.20, 158.1, 139.35, 135.5, 134.5, 130.87, 129.0, 128.96, 128.3, 125.8, 123.0, 86.9, 81.27, 75.8, 56.8, 56.3, 50.1, 42.4, 39.8, 39.6, 37.8, 37.0, 36.6, $36.3,35.9,32.0,31.99,28.3,28.1,27.5,24.4,24.0,23.0,22.7,21.1,19.4,18.8,12.0$. HRMS (ESI) m/z: $\quad[\mathrm{M}+\mathrm{Na}]^{+}$Calcd for $\mathrm{C}_{43} \mathrm{H}_{58} \mathrm{NaO}_{3} \mathrm{~S}$ 677.3999; Found 677.4006. FTIR (cm1) $3436,2936,1731,1683,1469,1440,1377,1277,1066$.

\section{(R)-2-((Tert-butoxycarbonyl)amino)-3-phenylpropyl-2-(phenylthio)-2-((1} phenylvinyl)oxy)acetate (3ae)

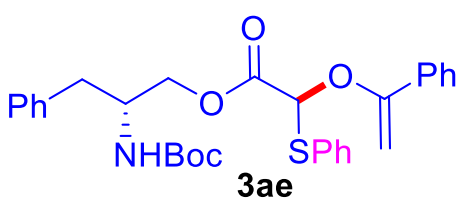

Following the general procedure, treatment of 2-(trimethylsilyl) phenyltrifluoromethanesulfonate $2 \mathrm{a}(0.90 \mathrm{~g}, 73.0 \mu \mathrm{L}, 0.3 \mathrm{mmol})$ with (R)-2-((tert-butoxycarbonyl)amino)-3-phenylpropyl 2-((2oxo-2-phenylethyl)thio)acetate 1ae $(0.111 \mathrm{~g}, 0.25 \mathrm{mmol})$ in the presence of KF $(0.035 \mathrm{~g}, 0.6 \mathrm{mmol})$ and 18-crown-6 $(0.158 \mathrm{~g}, 0.6 \mathrm{mmol})$ in THF $(1.0 \mathrm{~mL})$ at 25 ${ }^{\circ} \mathrm{C}$ for $0.5 \mathrm{~h}$ followed by flash column chromatography (Pet.ether/EtOAc $=97 / 03$ ) of the crude reaction mixture using silica gel afforded $(R)-2-(($ tert-butoxycarbonyl)amino)-3-phenylpropyl-2(phenylthio)-2-((1 phenylvinyl)oxy)acetate 3ae as a colourless oil $(0.115 \mathrm{~g}, 88 \%$ yield; diastereoselectivity determined by crude ${ }^{1} \mathrm{H}$ NMR is $\left.1: 1\right)$. 
$\boldsymbol{R}_{\mathbf{f}}($ Pet. ether $/ \mathrm{EtOAc}=95 / 05): 0.38 ;{ }^{1} \mathbf{H}$ NMR of one Diastereomer (400 $\left.\mathbf{~ M H z , ~ C D C l} \mathbf{3}\right) \delta 7.71$ $7.68(\mathrm{~m}, 2 \mathrm{H}), 7.64-7.61(\mathrm{~m}, 2 \mathrm{H}), 7.39-7.35(\mathrm{~m}, 6 \mathrm{H}), 7.31-7.22(\mathrm{~m}, 3 \mathrm{H}), 7.13-7.09(\mathrm{~m}, 2 \mathrm{H}), 5.81$ (s, 1H), 4.98-4.96 (m, 1H), 4.62-4.47 (m, 1H), 4.38 (d, J = 3.7 Hz, 1H), 4.11-3.99 (m, 2H), 3.96$3.92(\mathrm{~m}, 1 \mathrm{H}), 2.78-2.62(\mathrm{~m}, 2 \mathrm{H}), 1.42(\mathrm{~s}, 9 \mathrm{H}) .{ }^{13} \mathbf{C}$ NMR of one Diastereomer (100 $\left.\mathbf{M H z}, \mathbf{C D C l}_{3}\right)$ $\delta 166.8,157.8,155.1,137.1,135.1,134.6,130.5,129.3,129.30,129.2,129.1,128.4,126.7,125.6$,

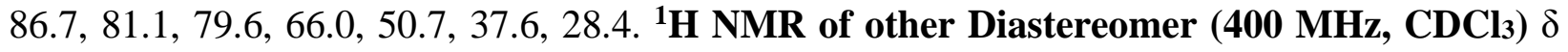
7.71-7.68 (m, 2H), 7.64-7.61 (m, 2H), 7.39-7.35 (m, 6H), 7.31-7.22 (m, 3H), 7.13-7.09 (m, 2H), $5.80(\mathrm{~s}, 1 \mathrm{H}), 4.98-4.96(\mathrm{~m}, 1 \mathrm{H}), 4.62-4.47(\mathrm{~m}, 1 \mathrm{H}), 4.41(\mathrm{~d}, J=3.7 \mathrm{~Hz}, 1 \mathrm{H}), 4.24-4.21(\mathrm{~m}, 1 \mathrm{H})$, 4.11-3.99 (m, 2H), 2.78-2.62 (m, 2H), 1.42 (s, 9H). ${ }^{13} \mathbf{C}$ NMR of other Diastereomer (100 MHz, $\left.\mathbf{C D C l}_{3}\right) \delta 166.78,158.0,155.01,137.2,135.2,134.7,130.4,129.4,129.27,129.2,129.09,128.40$, 126.7, 125.6, 86.9, 81.0, 79.57, 65.8, 50.7, 37.7, 28.41 HRMS (ESI) m/z: [M+Na $]^{+}$Calcd for $\mathrm{C}_{30} \mathrm{H}_{33} \mathrm{NNaO}_{5} \mathrm{~S}$ 542.1972; Found 542.1978. FTIR (cm $\left.{ }^{-1}\right)$ 3424, 2975, 2927, 1754, 1711, 1498, $1276,1236,1165,1023$.

\section{$N, N$-Dimethyl-2-(phenylthio)-2-((1-phenylvinyl)oxy)acetamide (3af)}

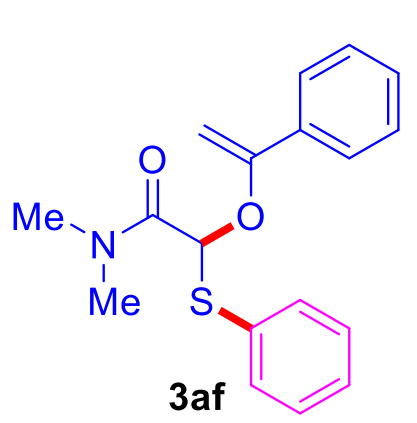

Following the general procedure, treatment of 2-(trimethylsilyl) phenyltrifluoromethanesulfonate $\mathbf{2 a}(0.090 \mathrm{~g}, 73 \mu \mathrm{L}, 0.3 \mathrm{mmol})$ with $N, N$-dimethyl-2-((2-oxo-2-phenylethyl)thio)acetamide 1af (0.059 g, $0.25 \mathrm{mmol})$ in the presence of $\mathrm{KF}(0.035 \mathrm{~g}, 0.6 \mathrm{mmol})$ and 18-crown-6 $(0.158 \mathrm{~g}, 0.6 \mathrm{mmol})$ in THF $(1.0 \mathrm{~mL})$ at $25^{\circ} \mathrm{C}$ for $2 \mathrm{~h}$ followed by flash column chromatography (Pet.ether/EtOAc $=90 / 10$ ) of the crude

reaction mixture using silica gel afforded $N, N$-Dimethyl-2-(phenylthio)-2-((1phenylvinyl)oxy)acetamide 3af as a pale yellow oil (0.062 g, 79\% yield).

$\boldsymbol{R}_{\mathbf{f}}($ Pet. ether $/$ EtOAc $=90 / 10): 0.25 ;{ }^{1} \mathbf{H}$ NMR (400 MHz, CDCl3) $\delta$ 7.62-7.59 (m, 2H), 7.58-7.54 (m, 2H), 7.36-7.30 (m, 6H), $5.93(\mathrm{~s}, 1 \mathrm{H}), 4.82(\mathrm{~d}, J=3.7 \mathrm{~Hz}, 1 \mathrm{H}), 4.31(\mathrm{~d}, J=3.6 \mathrm{~Hz}, 1 \mathrm{H}), 3.20$

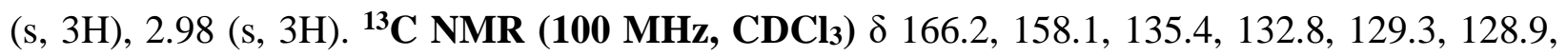
128.6, 128.4, 125.6, 86.9, 85.1, 37.3, 36.5. HRMS (ESI) m/z: $[\mathrm{M}+\mathrm{Na}]^{+}$Calcd for $\mathrm{C}_{18} \mathrm{H}_{19} \mathrm{NNaO}_{2} \mathrm{~S}$ 336.1029; Found: 336.1036. FTIR (cm-1) 3419, 2924, 1725, 1647, 1219, 1022. 


\section{$N$-Methoxy- $N$-methyl-2-(phenylthio)-2-((1-phenylvinyl)oxy)acetamide (3ag)}

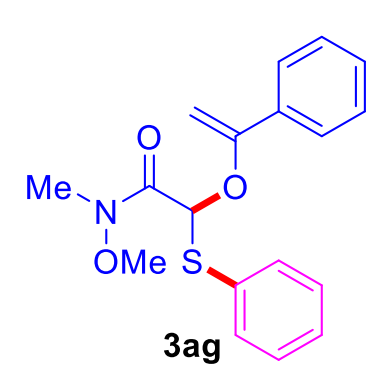

mixture using silica gel afforded $N$-methoxy- $N$-methyl-2-(phenylthio)-2-((1-

phenylvinyl)oxy)acetamide 3ag as a pale yellow oil (0.137 g, 83\% yield).

$\boldsymbol{R}_{\mathbf{f}}\left(\right.$ Pet. ether $/$ EtOAc $=$ 80/20): 0.46; ${ }^{1} \mathbf{H}$ NMR (400 MHz, CDCl $) \delta$ 7.67-7.65 (m, 2H), 7.59-7.55 (m, 2H), 7.37-7.31 (m, 6H), $6.21(\mathrm{~s}, 1 \mathrm{H}), 4.85(\mathrm{~d}, J=3.5 \mathrm{~Hz}, 1 \mathrm{H}), 4.33(\mathrm{~d}, J=3.5 \mathrm{~Hz}, 1 \mathrm{H}), 3.82$ (s, 3H), 3.24 (s, 3H). ${ }^{13}$ C NMR (100 MHz, CDCl3) $\delta$ 167.2, 158.3, 135.4, 133.2, 132.2, 129.1, 128.8, 128.6, 128.2, 125.8, 86.6, 80.3, 61.6, 32.7. HRMS (ESI) m/z: $[\mathrm{M}+\mathrm{Na}]^{+}$Calcd for $\mathrm{C}_{18} \mathrm{H}_{19} \mathrm{NNaO}_{3} \mathrm{~S}$ 352.0978; Found: 352.0982. FTIR (cm-1) 3427, 2936, 1663, 1477, 1441, 1064.

\section{Ethyl-2-(cyclohex-1-en-1-yloxy)-2-(phenylthio)acetate (3ah)}

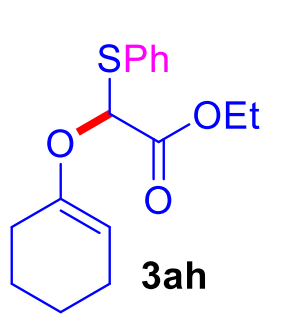

Following the general procedure, treatment of 2-(trimethylsilyl) phenyltrifluoromethanesulfonate $2 \mathbf{a}(0.179 \mathrm{~g}, 146 \mu \mathrm{L}, 0.6 \mathrm{mmol})$ with ethyl 2((2-oxocyclohexyl)thio)acetate 1ah $(0.108 \mathrm{~g}, 0.5 \mathrm{mmol})$ in the presence of $\mathrm{KF}$ $(0.070 \mathrm{~g}, 1.2 \mathrm{mmol})$ and 18-crown-6 $(0.317 \mathrm{~g}, 1.2 \mathrm{mmol})$ in THF $(2.0 \mathrm{~mL})$ at 25 ${ }^{\circ} \mathrm{C}$ for $1 \mathrm{~h}$ followed by flash column chromatography (Pet.ether/EtOAc $=97 / 03$ ) of the crude reaction mixture using silica gel afforded ethyl-2-(cyclohex-1-en-1-yloxy)-2(phenylthio)acetate 3ah as a colourless oil ( $0.075 \mathrm{~g}, 51 \%$ yield).

$\boldsymbol{R}_{\mathbf{f}}($ Pet. ether $/$ EtOAc $=95 / 05)$ : 0.30; ${ }^{1} \mathbf{H}$ NMR (400 MHz, CDCl 3$) \delta$ 7.54-7.40 (m, 2H), 7.35-7.28 (m, 3H), $5.54(\mathrm{~s}, 1 \mathrm{H}), 4.74(\mathrm{t}, J=3.9 \mathrm{~Hz}, 1 \mathrm{H}), 4.17-4.09(\mathrm{~m}, 2 \mathrm{H}), 3.21-2.11(\mathrm{~m}, 2 \mathrm{H}), 2.07-1.97$ $(\mathrm{m}, 2 \mathrm{H}), 1.71-1.65(\mathrm{~m}, 2 \mathrm{H}), 1.56-1.50(\mathrm{~m}, 2 \mathrm{H}), 1.19(\mathrm{t}, J=7.1 \mathrm{~Hz}, 3 \mathrm{H}) .{ }^{13} \mathrm{C}$ NMR (100 MHz, CDCl$\left._{3}\right) \delta 167.4,152.8,134.2,131.3,129.0,128.8,99.3,80.2,61.8,27.6,23.6,22.8,22.4,14.1$. HRMS (ESI) m/z; [M+Na] ${ }^{+}$Calcd for $\mathrm{C}_{16} \mathrm{H}_{20} \mathrm{NaO}_{3} \mathrm{~S}$ 315.1025; Found: 315.1031. FTIR (cm1) 2931, 1755, 1732, 1668, 1440, 1265, 1153. 


\section{Ethyl 2-(cyclohept-1-en-1-yloxy)-2-(phenylthio)acetate (3ai)}

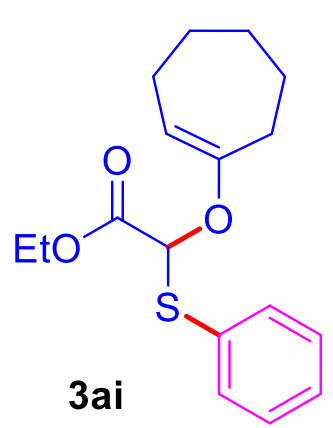

Following the general procedure, treatment of 2-(trimethylsilyl) phenyltrifluoromethanesulfonate $\mathbf{2 a}(0.179 \mathrm{~g}, 146 \mu \mathrm{L}, 0.6 \mathrm{mmol})$ with ethyl 2-((2-oxocycloheptyl)thio)acetate 1ai $(0.115 \mathrm{~g}, 0.5 \mathrm{mmol})$ in the presence of $\mathrm{KF}(0.070 \mathrm{~g}, 1.2 \mathrm{mmol})$ and 18-crown-6 (0.317 $\mathrm{g}, 1.2 \mathrm{mmol})$ in THF (2.0 $\mathrm{mL})$ at $25{ }^{\circ} \mathrm{C}$ for $4 \mathrm{~h}$ followed by flash column chromatography (Pet.ether/EtOAc $=97 / 03)$ of the crude reaction mixture using silica gel afforded ethyl 2-(cyclohept-1-en-1-yloxy)-2-(phenylthio)acetate 3ai as a colorless oil ( $0.101 \mathrm{~g}, 66 \%$ yield).

$\boldsymbol{R}_{\mathbf{f}}$ (Pet. ether /EtOAc = 95/05): 0.50; ${ }^{1} \mathbf{H}$ NMR (400 MHz, $\left.\mathbf{C D C l}_{3}\right) \delta$ 7.53-7.50 (m, 2H), 7.31-7.30 (m, 3H), $5.43(\mathrm{~s}, 1 \mathrm{H}), 4.83(\mathrm{t}, J=6.7 \mathrm{~Hz}, 1 \mathrm{H}), 4.16-4.10(\mathrm{~m}, 2 \mathrm{H}), 2.36-2.33(\mathrm{~m}, 2 \mathrm{H}), 2.06-2.02$ (m, 2H), 1.72-1.66 (m, 2H), 1.57-1.44 (m, 4H), 1.18 (t, $J=7.1 \mathrm{~Hz}, 3 \mathrm{H}) .{ }^{13} \mathrm{C}$ NMR (100 MHz, CDCl3) $\delta 167.3,158.9,134.1,131.4,128.9,128.7,102.7,80.5,61.7,33.3,31.8,27.6,25.3,14.1$. HRMS (ESI) m/z: [M+Na] ${ }^{+}$Calcd for $\mathrm{C}_{17} \mathrm{H}_{22} \mathrm{NaO}_{3} \mathrm{~S}$ 329.1187; Found 329.1187. FTIR (cm1) $2923,2846,1756,1662,1164,1026$.

\section{Ethyl-2-((1H-inden-3-yl)oxy)-2-(phenylthio)acetate (3aj)}

Following the general procedure, treatment of 2-(trimethylsilyl)

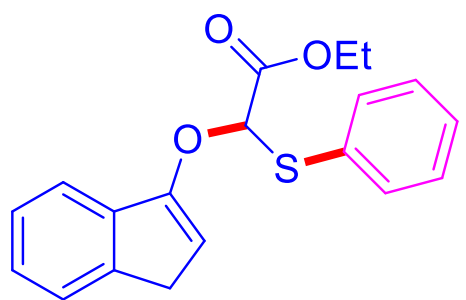

3aj phenyltrifluoromethanesulfonate 2a $(0.179 \mathrm{~g}, 146 \mu \mathrm{L}, 0.6 \mathrm{mmol})$ with ethyl 2-((1-oxo-2,3-dihydro-1H-inden-2-yl)thio)acetate 1aj $(0.125 \mathrm{~g}, 0.5 \mathrm{mmol})$ in the presence of $\mathrm{KF}(0.070 \mathrm{~g}, 1.2 \mathrm{mmol})$ and 18-crown-6 (0.317 g, $1.2 \mathrm{mmol})$ in THF $(2.0 \mathrm{~mL})$ at $25{ }^{\circ} \mathrm{C}$ for $1 \mathrm{~h}$ followed by flash column chromatography (Pet.ether/EtOAc $=$ 97/03) of the crude reaction mixture using silica gel afforded ethyl-2-((1H-inden-3-yl)oxy)-2(phenylthio)acetate 3aj as a colourless oil $(0.128 \mathrm{~g}, 79 \%$ yield).

$\boldsymbol{R}_{\mathbf{f}}\left(\right.$ Pet. ether /EtOAc = 95/05): 0.46; ${ }^{1} \mathbf{H}$ NMR (400 MHz, CDCl3) $\delta$ 7.64-7.60 (m, 3H), 7.46-7.44 $(\mathrm{m}, 1 \mathrm{H}), 7.40-7.33(\mathrm{~m}, 4 \mathrm{H}), 7.32-7.28(\mathrm{~m}, 1 \mathrm{H}), 5.86(\mathrm{~s}, 1 \mathrm{H}), 5.41(\mathrm{t}, J=2.3 \mathrm{~Hz}, 1 \mathrm{H}), 4.20(\mathrm{q}, J=$ $7.1 \mathrm{~Hz}, 2 \mathrm{H}), 3.43-3.30(\mathrm{~m}, 2 \mathrm{H}), 1.23(\mathrm{t}, J=7.1 \mathrm{~Hz}, 3 \mathrm{H}) .{ }^{13} \mathbf{C}$ NMR (100 MHz, CDCl3) $\delta 166.5$, $155.1,142.5,139.2,134.3,130.7,129.0,128.96,126.2,125.8,123.9,118.5,101.9,82.6,61.9$, 34.6, 14.0. HRMS (ESI) m/z: [M+Na] ${ }^{+}$Calcd for $\mathrm{C}_{19} \mathrm{H}_{18} \mathrm{NaO}_{3} \mathrm{~S}$ 349.0869; Found 349.0870. FTIR (cm $\left.{ }^{-1}\right)$ 3400, 2927, 1741, 1709, 1279, 1068. 


\section{Ethyl-2-((3,4-dihydronaphthalen-1-yl)oxy)-2-(phenylthio)acetate (3ak)}

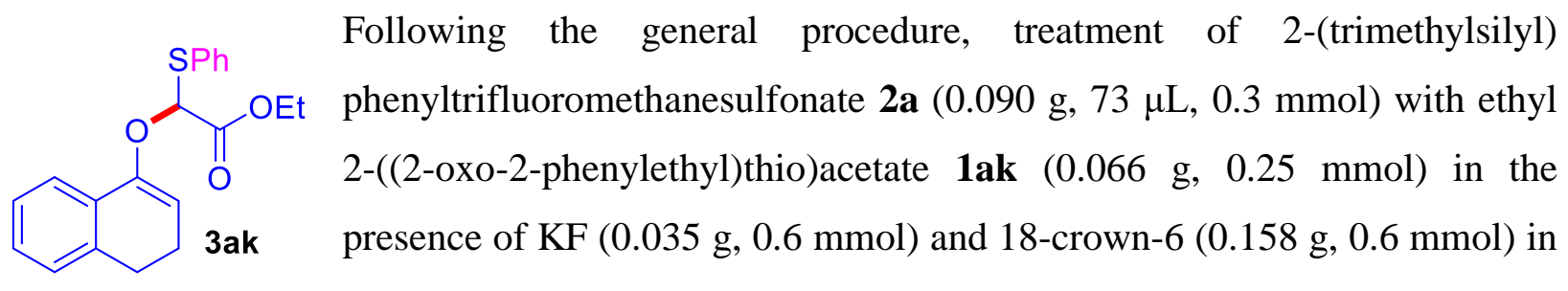

THF $(1.0 \mathrm{~mL})$ at $25{ }^{\circ} \mathrm{C}$ for $2 \mathrm{~h}$ followed by flash column chromatography (Pet.ether/EtOAc = 97/03) of the crude reaction mixture using silica gel afforded ethyl-2-((3,4-dihydronaphthalen-1yl)oxy)-2-(phenylthio)acetate 3ak as a colourless oil (0.046 g, 54\% yield).

$\boldsymbol{R}_{\mathbf{f}}($ Pet. ether $/ \mathrm{EtOAc}=95 / 05): 0.35 ;{ }^{1} \mathbf{H}$ NMR (400 MHz, CDCl$) \delta ~ 7.61-7.57$ (m, 3H), 7.38-7.32 (m, 3H), 7.27-7.20 (m, 2H), 7.17-7.15 (m, 1H), $5.73(\mathrm{~s}, 1 \mathrm{H}), 5.15$ (t, J=4.7 Hz, 1H), 4.23-4.12 $(\mathrm{m}, 2 \mathrm{H}), 2.86-2.74(\mathrm{~m}, 2 \mathrm{H}), 2.46-2.31(\mathrm{~m}, 2 \mathrm{H}), 1.21(\mathrm{t}, J=7.1 \mathrm{~Hz}, 3 \mathrm{H}) .{ }^{13} \mathrm{C}$ NMR (100 MHz, CDCl3) $\delta 167.1,150.0,137.0,134.6,131.2,130.9,129.1,129.0,128.0,127.2,126.5,122.1,101.0$, 81.0, 61.9, 28.0, 22.0, 14.1. HRMS (ESI) m/z: [M+Na] ${ }^{+}$Calcd for $\mathrm{C}_{20} \mathrm{H}_{20} \mathrm{NaO}_{3} \mathrm{~S} 363.1025$; Found 363.1028. FTIR (cm-1) 2930, 1736, 1680, 1286, 1185, 1089, 1022.

\section{Ethyl-2-(phenylthio)-2-(prop-1-en-2-yloxy)acetate (3al)}

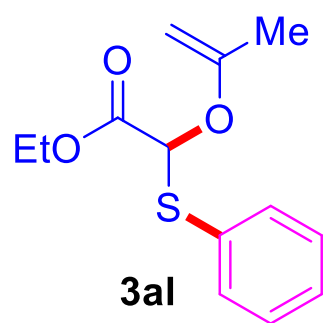

Following the general procedure, treatment of 2-(trimethylsilyl) phenyltrifluoromethanesulfonate $2 \mathbf{a}(0.179 \mathrm{~g}, 146 \mu \mathrm{L}, 0.6 \mathrm{mmol})$ with ethyl 2-((2-oxopropyl)thio)acetate 1al $(0.088 \mathrm{~g}, 0.5 \mathrm{mmol})$ in the presence of $\mathrm{KF}$ (0.070 g, $1.2 \mathrm{mmol})$ and 18-crown-6 (0.317 g, $1.2 \mathrm{mmol})$ in THF (2.0 mL) at $25^{\circ} \mathrm{C}$ for $1 \mathrm{~h}$ followed by flash column chromatography (Pet.ether/EtOAc $=97 / 03)$ of the crude reaction mixture using silica gel afforded ethyl-2(phenylthio)-2-(prop-1-en-2-yloxy)acetate 3al as a colourless oil ( $0.120 \mathrm{~g}, 96 \%$ yield).

$\boldsymbol{R}_{\mathbf{f}}\left(\right.$ Pet. ether /EtOAc = 95/05): 0.48; ${ }^{1} \mathbf{H}$ NMR (400 MHz, CDCl3) $\delta$ 7.54-7.51 (m, 2H), 7.36-7.28 $(\mathrm{m}, 3 \mathrm{H}), 5.52(\mathrm{~s}, 1 \mathrm{H}), 4.16-4.10(\mathrm{~m}, 3 \mathrm{H}), 3.92(\mathrm{~d}, J=2.9 \mathrm{~Hz}, 1 \mathrm{H}), 1.91(\mathrm{~s}, 3 \mathrm{H}), 1.19(\mathrm{t}, J=7.1 \mathrm{~Hz}$, 3H). ${ }^{13}$ C NMR (100 MHz, CDCl3) $\delta 166.9,157.6,134.2,131.1,129.0,128.9,85.8,80.6,61.8$, 20.9, 14.1. HRMS (ESI) m/z: $[\mathrm{M}+\mathrm{Na}]^{+}$Calcd for $\mathrm{C}_{13} \mathrm{H}_{16} \mathrm{NaO}_{3} \mathrm{~S}$ 275.0712; Found 275.0716. FTIR $\left(\mathbf{c m}^{-1}\right)$ 3370, 2977, 1740, 1709, 1278, 1165, 1021 
Ethyl-2-((3,4-dimethylphenyl)thio)-2-((1-phenylvinyl)oxy)acetate (3am)<smiles>C=C(OC(Sc1ccc(C)c(C)c1)C(=O)OCC)c1ccccc1</smiles>

Following the general procedure, treatment of 4,5-dimethyl-2(trimethylsilyl)phenyl trifluoromethanesulfonate $\mathbf{2 b}(0.098$ g, 0.3 mmol) with ethyl 2-((2-oxo-2-phenylethyl)thio)acetate $\mathbf{1 a}(0.60 \mathrm{~g}, 0.25$ $\mathrm{mmol})$ in the presence of $\mathrm{KF}(0.035 \mathrm{~g}, 0.6 \mathrm{mmol})$ and 18-crown-6 $(0.158 \mathrm{~g}, 0.6 \mathrm{mmol})$ in THF $(1.0 \mathrm{~mL})$ at $25^{\circ} \mathrm{C}$ for $5 \mathrm{~h}$ followed by flash column chromatography (Pet.ether/EtOAc $=97 / 03$ ) of the crude reaction mixture using silica gel afforded ethyl-2-((3,4dimethylphenyl)thio)-2-((1-phenylvinyl)oxy)acetate 3am as a colourless oil (0.079 g, 92\% yield). $\boldsymbol{R}_{\mathbf{f}}($ Pet. ether $/$ EtOAc $=95 / 05): 0.49 ;{ }^{1} \mathbf{H}$ NMR (400 MHz, CDCl $\left.\mathbf{3}\right) \delta$ 7.67-7.63 (m, 2H), 7.38-7.30 (m, 5H), $7.09(\mathrm{~d}, J=7.8 \mathrm{~Hz}, 1 \mathrm{H}), 5.67(\mathrm{~s}, 1 \mathrm{H}), 4.90(\mathrm{~d}, J=3.6 \mathrm{~Hz}, 1 \mathrm{H}), 4.33$ (d, $J=3.6 \mathrm{~Hz}, 1 \mathrm{H})$, 4.22-4.12 (m, 2H), $2.26(\mathrm{~s}, 3 \mathrm{H}), 2.22(\mathrm{~s}, 3 \mathrm{H}), 1.22$ (t, $J=7.1 \mathrm{~Hz}, 3 \mathrm{H}) .{ }^{13} \mathbf{C}$ NMR (100 MHz, CDCl3) $\delta 167.0,157.9,138.2,137.4,136.2,135.5,132.3,130.3,128.9,128.3,127.0,125.8,86.9$, 81.4, 61.8, 19.7, 14.1. HRMS (ESI) m/z; [M+K] ${ }^{+}$Calcd for $\mathrm{C}_{20} \mathrm{H}_{22} \mathrm{KO}_{3} \mathrm{~S}$ 381.0921; Found: 381.0927. FTIR (cm-1) 3461, 2981, 2366, 1736, 1682, 1269, 1074, 1022.

\section{Ethyl-2-(benzo[d][1,3]dioxol-5-ylthio)-2-((1-phenylvinyl)oxy)acetate (3an)}

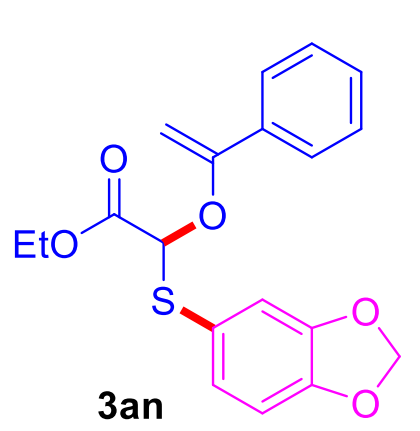

Following the general procedure, treatment of 6(trimethylsilyl)benzo[d][1,3]dioxol-5-yl trifluoromethanesulfonate 2c $(0.103 \mathrm{~g}, 0.3 \mathrm{mmol})$ with ethyl 2-((2-oxo-2-phenylethyl)thio)acetate 1a $(0.60 \mathrm{~g}, 0.25 \mathrm{mmol})$ in the presence of $\mathrm{KF}(0.035 \mathrm{~g}, 0.6 \mathrm{mmol})$ and 18 crown-6 $(0.158 \mathrm{~g}, 0.6 \mathrm{mmol})$ in THF $(1.0 \mathrm{~mL})$ at $25^{\circ} \mathrm{C}$ for $1 \mathrm{~h}$ followed by flash column chromatography (Pet.ether/EtOAc $=93 / 07$ ) of the crude reaction mixture using silica gel afforded ethyl-2(benzo[d][1,3]dioxol-5-ylthio)-2-((1-phenylvinyl)oxy)acetate 3an as a colourless oil (0.081 g, $90 \%$ yield).

$\boldsymbol{R}_{\mathbf{f}}($ Pet. ether $/$ EtOAc $=95 / 05)$ : 0.18; ${ }^{1} \mathbf{H}$ NMR (400 MHz, CDCl3) $\delta$ 7.67-7.63 (m, 2H), 7.38-7.32 $(\mathrm{m}, 3 \mathrm{H}), 7.09-7.04(\mathrm{~m}, 2 \mathrm{H}), 6.76(\mathrm{~d}, J=8.0 \mathrm{~Hz}, 1 \mathrm{H}), 5.97(\mathrm{~s}, 2 \mathrm{H}), 5.63(\mathrm{~s}, 1 \mathrm{H}), 4.90(\mathrm{~d}, J=3.6$ $\mathrm{Hz}, 1 \mathrm{H}), 4.33$ (d, $J=3.6 \mathrm{~Hz}, 1 \mathrm{H}), 4.19$ (q, $J=7.1 \mathrm{~Hz}, 2 \mathrm{H}), 1.24(\mathrm{t}, J=7.1 \mathrm{~Hz}, 3 \mathrm{H}) .{ }^{13} \mathbf{C}$ NMR (100 MHz, CDCl3) $\delta 166.8,157.9,149.2,147.9,135.4,130.0,129.0,128.3,125.8,121.9,115.6$, 
108.7, 101.6, 86.9, 81.4, 61.9, 14.2. HRMS (ESI) m/z: $[\mathrm{M}+\mathrm{Na}]^{+}$Calcd for $\mathrm{C}_{19} \mathrm{H}_{18} \mathrm{NaO}_{5} \mathrm{~S}$ 381.0767; Found 381.0773. FTIR (cm-1) 3441, 2982, 1733, 1681, 1476, 1235, 1078, 932.

\section{Ethyl-2-((3,4-difluorophenyl)thio)-2-((1-phenylvinyl)oxy)acetate (3ao)}<smiles>C=C(OC(Sc1ccc(F)c(F)c1)C(=O)OCC)c1ccccc1</smiles>

Following the general procedure, treatment of 4,5-difluoro-2(trimethylsilyl)phenyl trifluoromethanesulfonate $\mathbf{2 d}(0.100 \mathrm{~g}, 0.3 \mathrm{mmol})$ with ethyl 2-((2-oxo-2-phenylethyl)thio)acetate 1a $(0.60 \mathrm{~g}, 0.25 \mathrm{mmol})$ in the presence of $\mathrm{KF}(0.035 \mathrm{~g}, 0.6 \mathrm{mmol})$ and 18 -crown-6 $(0.158 \mathrm{~g}, 0.6 \mathrm{mmol})$ in THF $(1.0 \mathrm{~mL})$ at $25^{\circ} \mathrm{C}$ for $6 \mathrm{~h}$ followed by flash column chromatography (Pet.ether/EtOAc $=97 / 03)$ of the crude reaction mixture using silica gel $(0.069 \mathrm{~g}, 78 \%$ yield $)$.

$\boldsymbol{R}_{\mathbf{f}}\left(\right.$ Pet. ether $/$ EtOAc = 95/05): 0.50; ${ }^{1} \mathbf{H}$ NMR (400 MHz, $\left.\mathbf{C D C l}_{3}\right) \delta$ 7.64-7.59 (m, 2H), 7.43-7.34 $(\mathrm{m}, 4 \mathrm{H}), 7.32-7.28(\mathrm{~m}, 1 \mathrm{H}), 7.15-7.08(\mathrm{~m}, 1 \mathrm{H}), 5.67(\mathrm{~s}, 1 \mathrm{H}), 4.93(\mathrm{~d}, J=3.7 \mathrm{~Hz}, 1 \mathrm{H}), 4.35(\mathrm{~d}, J=$ $3.7 \mathrm{~Hz}, 1 \mathrm{H}), 4.22-4.15(\mathrm{~m}, 2 \mathrm{H}), 1.23(\mathrm{t}, J=7.1 \mathrm{~Hz}, 3 \mathrm{H}) .{ }^{13} \mathbf{C}$ NMR (100 MHz, CDCl$) \delta 166.5$, $157.8,151.5\left(\mathrm{dd}, J_{l}=253.7 \mathrm{~Hz}, J_{2}=14.8 \mathrm{~Hz}\right), 150.1\left(\mathrm{dd}, J_{l}=255.1 \mathrm{~Hz}, J_{2}=16.1 \mathrm{~Hz}\right), 135.2$, 131.7, $\left(\mathrm{dd}, J_{l}=6.3 \mathrm{~Hz}, J_{2}=3.8 \mathrm{~Hz}\right), 129.2,128.5,126.4\left(\mathrm{dd}, J_{1}=5.9 \mathrm{~Hz}, J_{2}=4.3 \mathrm{~Hz}\right), 125.7$, $124.1(\mathrm{~d}, J=17.4 \mathrm{~Hz}), 117.8(\mathrm{~d}, J=17.3 \mathrm{~Hz}), 87.3,80.6,62.1,14.2 . \mathbf{H R M S}(\mathbf{E S I}) \mathrm{m} / \mathrm{z}:[\mathrm{M}+\mathrm{Na}]^{+}$ Calcd for $\mathrm{C}_{18} \mathrm{H}_{16} \mathrm{~F}_{2} \mathrm{NaO}_{3} \mathrm{~S}$ 373.0680; Found 373.0684. FTIR (cm 1) $3441,2986,1736,1681,1601,1502,1273,1090$.

\section{Ethyl-2-((2,3-dihydro-1H-inden-5-yl)thio)-2-((1-phenylvinyl)oxy)acetate (3ap)}

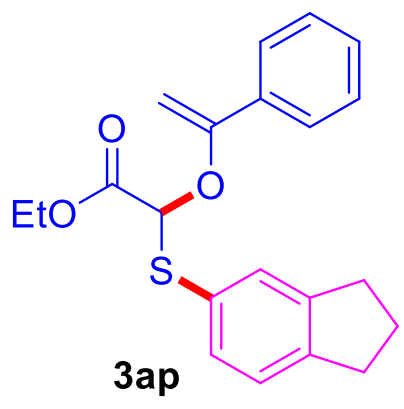

Following the general procedure, treatment of 6-(trimethylsilyl)-2,3dihydro- $1 H$-inden-5-yl trifluoromethanesulfonate 2 e $(0.102 \mathrm{~g}, 0.3$ mmol $)$ with ethyl 2-((2-oxo-2-phenylethyl)thio)acetate $1 \mathbf{a}(0.60 \mathrm{~g}, 0.25$ $\mathrm{mmol})$ in the presence of $\mathrm{KF}(0.035 \mathrm{~g}, 0.6 \mathrm{mmol})$ and 18-crown-6 $(0.158 \mathrm{~g}, 0.6 \mathrm{mmol})$ in $\mathrm{THF}(1.0 \mathrm{~mL})$ at $25^{\circ} \mathrm{C}$ for $5 \mathrm{~h}$ followed by flash column chromatography (Pet.ether/EtOAc = 97/03) of the crude reaction mixture using silica gel afforded ethyl-2-((2,3-dihydro- $1 H$ inden-5-yl)thio)-2-((1-phenylvinyl)oxy)acetate 3ap as a colourless oil (0.082 g, 92\% yield). 
$\boldsymbol{R}_{\mathbf{f}}\left(\right.$ Pet. ether $/$ EtOAc = 95/05): 0.35; ${ }^{1} \mathbf{H}$ NMR (400 MHz, CDCl $) \delta$ 7.67-7.62 (m, 2H), $7.43(\mathrm{~s}$, 1H), 7.38-7.32 (m, 4H), 7.18 (d, J=7.7 Hz, 1H), 5.67 (s, 1H), 4.89 (d, J = 3.6 Hz, 1H), 4.33 (d, $J$ $=3.6 \mathrm{~Hz}, 1 \mathrm{H}), 4.18(\mathrm{q}, \mathrm{J}=7.1 \mathrm{~Hz}, 2 \mathrm{H}), 2.89\left(\mathrm{dd}, J_{1}=16.0 \mathrm{~Hz}, J_{2}=7.9 \mathrm{~Hz}, 4 \mathrm{H}\right), 2.08(\mathrm{p}, J=7.5$ Hz, 2H), 1.22 (t, $J=7.1 \mathrm{~Hz}, 3 \mathrm{H}) .{ }^{13} \mathrm{C}$ NMR (100 MHz, CDCl3) $\delta$ 167.0, 158.0, 145.9, 145.3, 135.5, 133.0, 131.2, 128.9, 128.3, 127.4, 125.8, 124.9, 86.9, 81.6, 61.8, 32.8, 25.5, 14.1. HRMS (ESI) $\mathrm{m} / \mathrm{z}: \quad[\mathrm{M}+\mathrm{Na}]^{+}$Calcd for $\mathrm{C}_{21} \mathrm{H}_{22} \mathrm{NaO}_{3} \mathrm{~S}$ 377.1182; Found 377.1189. FTIR (cm1) $3436,2956,1736,1682,1269,1182,1073,862$.

Ethyl-2-((2,5-dimethylphenyl)thio)-2-((1-phenylvinyl)oxy)acetate (3aq)

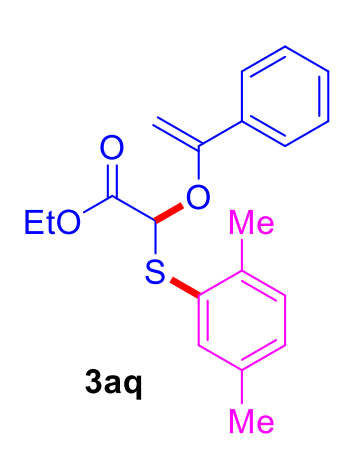

Following the general procedure, treatment of 3,6-dimethyl-2(trimethylsilyl)phenyl trifluoromethanesulfonate $\mathbf{2 f}(0.098 \mathrm{~g}, 0.3 \mathrm{mmol})$ with ethyl 2-((2-oxo-2-phenylethyl)thio)acetate $1 \mathrm{a}(0.60 \mathrm{~g}, 0.25 \mathrm{mmol})$ in the presence of KF $(0.035 \mathrm{~g}, 0.6 \mathrm{mmol})$ and 18-crown-6 $(0.158 \mathrm{~g}, 0.6 \mathrm{mmol})$ in THF (1.0 mL) at $25{ }^{\circ} \mathrm{C}$ for $1.5 \mathrm{~h}$ followed by flash column chromatography (Pet.ether/EtOAc $=97 / 03)$ of the crude reaction mixture using silica gel afforded ethyl-2-((2,5-dimethylphenyl)thio)-2-((1-phenylvinyl)oxy)acetate

3aq as a colourless oil (0.078 $\mathrm{g}, 91 \%$ yield $)$.

$\boldsymbol{R}_{\mathbf{f}}\left(\right.$ Pet. ether $/$ EtOAc = 95/05): 0.49; ${ }^{1} \mathbf{H}$ NMR (400 MHz, CDCl $) \delta$ 7.66-7.63 (m, 2H), $7.43(\mathrm{~s}$, 1H), 7.38-7.34 (m, 3H), $7.14(\mathrm{~d}, J=7.7 \mathrm{~Hz}, 1 \mathrm{H}), 7.08$ (d, J=7.7 Hz, 1H), $5.71(\mathrm{~s}, 1 \mathrm{H}), 4.88(\mathrm{~d}, J$ $=3.6 \mathrm{~Hz}, 1 \mathrm{H}), 4.31(\mathrm{~d}, J=3.6 \mathrm{~Hz}, 1 \mathrm{H}), 4.16(\mathrm{q}, J=7.2 \mathrm{~Hz}, 2 \mathrm{H}), 2.46(\mathrm{~s}, 3 \mathrm{H}), 2.28(\mathrm{~s}, 3 \mathrm{H}), 1.22$ $(\mathrm{t}, J=7.1 \mathrm{~Hz}, 3 \mathrm{H}) .{ }^{13} \mathbf{C}$ NMR (100 MHz, CDCl$) \delta ~ 167.1,158.1,139.0,136.6,136.1,135.5,130.3$, 130.2, 129.8, 128.9, 128.3, 125.8, 86.6, 81.5, 61.9, 20.8, 20.7, 14.0. HRMS (ESI) m/z: [M+K] ${ }^{+}$ Calcd for $\mathrm{C}_{20} \mathrm{H}_{22} \mathrm{KO}_{3} \mathrm{~S}$ 381.0921; Found 381.0933. FTIR (cm 1) $3438,2982,2922,1737,1683,1488,1269,1182,1021$

\section{Ethyl-2-(naphthalen-2-ylthio)-2-((1-phenylvinyl)oxy)acetate (3ar)}

Following the general procedure, treatment of 3-(trimethylsilyl)naphthalen-2-yl trifluoromethanesulfonate $\mathbf{2 g}(0.105 \mathrm{~g}, 0.3 \mathrm{mmol})$ with ethyl 2-((2-oxo-2-phenylethyl)thio)acetate $1 \mathrm{a}(0.060 \mathrm{~g}, 0.25 \mathrm{mmol})$ in the presence of $\mathrm{KF}(0.035 \mathrm{~g}, 0.6 \mathrm{mmol})$ and 18-crown-6 $(0.158 \mathrm{~g}, 0.6$ $\mathrm{mmol})$ in $\mathrm{THF}(1.0 \mathrm{~mL})$ at $25^{\circ} \mathrm{C}$ for $6 \mathrm{~h}$ followed by flash column chromatography 
(Pet.ether/EtOAc $=97 / 03)$ of the crude reaction mixture using silica gel afforded ethyl-2(naphthalen-2-ylthio)-2-((1-phenylvinyl)oxy)acetate 3ar as a colourless oil (0.075 g, 82\% yield).<smiles>C=C(OC(Sc1ccc2ccccc2c1)C(=O)OCC)c1ccccc1</smiles>
$\boldsymbol{R}_{\mathbf{f}}(\mathrm{Pet}$. ether $/ \mathrm{EtOAc}=95 / 05): 0.37 ;{ }^{1} \mathbf{H}$ NMR $\left(\mathbf{4 0 0} \mathbf{~ M H z}, \mathbf{C D C l}_{3}\right) \delta$ 8.09 (s, $1 \mathrm{H}), 7.85-7.76(\mathrm{~m}, 3 \mathrm{H}), 7.69-7.63(\mathrm{~m}, 3 \mathrm{H}), 7.54-7.48(\mathrm{~m}, 2 \mathrm{H})$, 7.36- $7.34(\mathrm{~m}, 3 \mathrm{H}), 5.81(\mathrm{~s}, 1 \mathrm{H}), 4.93(\mathrm{~d}, J=3.7 \mathrm{~Hz}, 1 \mathrm{H}), 4.38(\mathrm{~d}, J=$ $3.7 \mathrm{~Hz}, 1 \mathrm{H}), 4.21-4.09(\mathrm{~m}, 2 \mathrm{H}), 1.15(\mathrm{t}, J=7.1 \mathrm{~Hz}, 3 \mathrm{H}) .{ }^{13} \mathbf{C}$ NMR (100 MHz, $\left.\mathbf{C D C l}_{3}\right) \delta$ 166.9, 158.0, 135.4, 134.3, 133.5, 133.3, 131.2, $129.0,128.6,128.4,128.1,127.9,127.8,127.0,126.7,125.8,87.1$, 81.4, 62.0, 14.1. HRMS (ESI) m/z: $[\mathrm{M}+\mathrm{Na}]^{+}$Calcd for $\mathrm{C}_{22} \mathrm{H}_{20} \mathrm{NaO}_{3} \mathrm{~S}$ 387.1025; Found 387.1031. FTIR (cm-1) 3437, 3054, 2363, 1733, 1622, 1272, 1065, 857.

Ethyl-2-(naphthalen-2-ylthio)-2-((1-phenylvinyl)oxy)acetate (3ar) and Ethyl 2-(naphthalen-1-ylthio)-2-((1-phenylvinyl)oxy)acetate (3as)

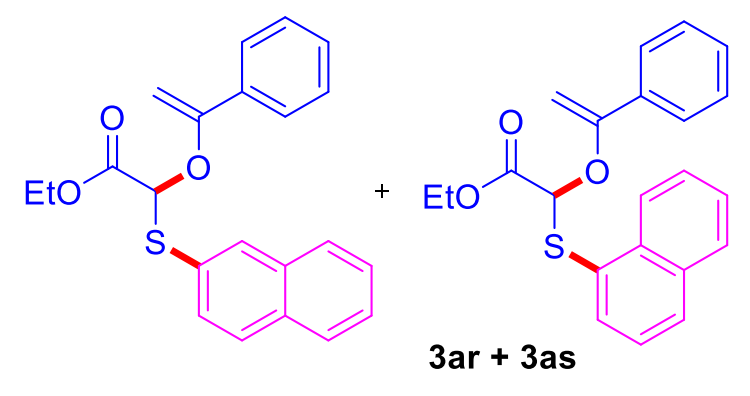

Following the general procedure, treatment of 1(trimethylsilyl)naphthalen-2-yl trifluoromethanesulfonate $\mathbf{2 h}(0.094 \mathrm{~g}, 0.3 \mathrm{mmol})$ with ethyl 2-((2-oxo-2-phenylethyl)thio)acetate 1a $(0.60 \mathrm{~g}, 0.25 \mathrm{mmol})$ in the presence of $\mathrm{KF}(0.035 \mathrm{~g}$, $0.6 \mathrm{mmol})$ and 18 -crown-6 (0.158 $\mathrm{g}, 0.6 \mathrm{mmol})$ in

THF $(1.0 \mathrm{~mL})$ at $25{ }^{\circ} \mathrm{C}$ for $2 \mathrm{~h}$ followed by flash column chromatography (Pet.ether/EtOAc $=$ 97/03) of the crude reaction mixture using silica gel afforded Ethyl-2-(naphthalen-2-ylthio)-2-((1phenylvinyl)oxy)acetate (3ar) and Ethyl 2-(naphthalen-1-ylthio)-2-((1-phenylvinyl)oxy)acetate (3as) as a mixture of regioisomers in 1.2:1 ratio as a colorless oil $(0.077 \mathrm{~g}, 84 \%)$.

$\boldsymbol{R}_{\mathbf{f}}($ Pet. ether $/ \mathrm{EtOAc}=95 / 05): 0.49 ;{ }^{1} \mathbf{H}$ NMR $(400 \mathrm{MHz}, \mathbf{C D C l} 3)$ of Major Isomer $(3 \mathrm{ar}) ; \delta$ $8.11(\mathrm{~s}, 1 \mathrm{H}), 7.92-7.77(\mathrm{~m}, 3 \mathrm{H}), 7.70-7.64(\mathrm{~m}, 3 \mathrm{H}), 7.61-7.50(\mathrm{~m}, 2 \mathrm{H}), 7.37-7.33(\mathrm{~m}, 3 \mathrm{H}), 5.83(\mathrm{~s}$, $1 \mathrm{H}), 4.95(\mathrm{~d}, J=3.7 \mathrm{~Hz}, 1 \mathrm{H}), 4.39$ (d, $J=3.7 \mathrm{~Hz}, 1 \mathrm{H}), 4.20-4.13(\mathrm{~m}, 2 \mathrm{H}), 1.16(\mathrm{t}, J=7.1 \mathrm{~Hz}, 3 \mathrm{H})$. ${ }^{13} \mathbf{C}$ NMR (100 MHz, CDCl $)$ of Major Isomer; $\delta 166.9,158.0,135.4,134.3,133.5,133.3,131.2$, 129.0, 128.6, 128.5, 128.1, 127.9, 127.8, 127.0, 126.7, 125.8, 87.1, 81.4, 62.0, 14.1. Representative peaks of Minor Isomer (3as); ${ }^{1} \mathrm{H}$ NMR (400 MHz, CDCl 3$) ; \delta 8.64-8.02(\mathrm{~m}$, 1H), $7.44(\mathrm{t}, J=7.7 \mathrm{~Hz}, 1 \mathrm{H}), 5.80(\mathrm{~s}, 1 \mathrm{H}), 4.90(\mathrm{~d}, J=3.7 \mathrm{~Hz}, 1 \mathrm{H}), 4.35(\mathrm{~d}, J=3.7 \mathrm{~Hz}, 1 \mathrm{H}), 4.01$ $3.91(\mathrm{~m}, 2 \mathrm{H}), 1.03(\mathrm{t}, J=7.1 \mathrm{~Hz}, 3 \mathrm{H}) .{ }^{\mathbf{1 3}} \mathbf{C} \mathbf{~ N M R}\left(\mathbf{1 0 0} \mathbf{~ M H z}, \mathbf{C D C l}_{\mathbf{3}} ; \delta 166.9,158.0,135.8,135.4\right.$, 135.3, 134.2, 130.6, 128.9, 128.5, 128.2, 127.0, 126.4, 126.3, 125.5, 86.7, 61.8, 13.8. HRMS 
(ESI) $\mathrm{m} / \mathrm{z}$ : $[\mathrm{M}+\mathrm{Na}]^{+}$Calcd for $\mathrm{C}_{22} \mathrm{H}_{20} \mathrm{NaO}_{3} \mathrm{~S}$ 387.1025; Found 387.1032. FTIR (cm1) $3055,2375,1753,1621,1276,1056,947$.

Ethyl-2-((3-methoxyphenyl)thio)-2-((1-phenylvinyl)oxy)acetate (3at)

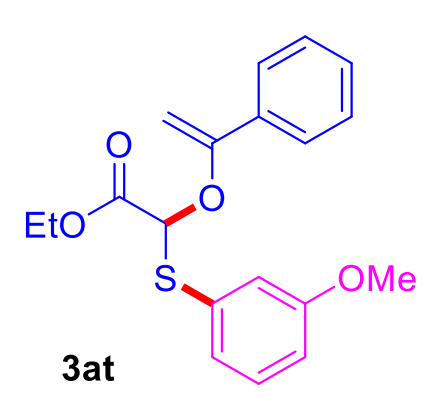

Following the general procedure, treatment of 3-methoxy-2(trimethylsilyl)phenyl trifluoromethanesulfonate $\mathbf{2 i}(0.099 \quad \mathrm{~g}, 0.3$ mmol) with ethyl 2-((2-oxo-2-phenylethyl)thio)acetate $1 \mathbf{a}(0.60 \mathrm{~g}, 0.25$ $\mathrm{mmol})$ in the presence of $\mathrm{KF}(0.035 \mathrm{~g}, 0.6 \mathrm{mmol})$ and 18-crown-6 $(0.158 \mathrm{~g}, 0.6 \mathrm{mmol})$ in THF $(1.0 \mathrm{~mL})$ at $25^{\circ} \mathrm{C}$ for $1.0 \mathrm{~h}$ followed by flash column chromatography (Pet.ether/EtOAc $=95 / 05)$ of the crude reaction mixture using silica gel afforded ethyl-2-((3-methoxyphenyl)thio)-2-((1phenylvinyl)oxy)acetate 3at as a colourless oil ( $0.073 \mathrm{~g}, 84 \%$ yield).

$\boldsymbol{R}_{\mathbf{f}}\left(\right.$ Pet. ether /EtOAc = 95/05): 0.29; ${ }^{1} \mathbf{H}$ NMR $\left(\mathbf{4 0 0} \mathbf{~ M H z}, \mathbf{C D C l}_{3}\right) \delta$ 7.67-7.54 (m, 2H), 7.38-7.34 (m, 3H), 7.25-7.21 (m, 1H), 7.15-7.11 (m, 2H), 6.90-6.88 (m, 1H), $5.74(\mathrm{~s}, 1 \mathrm{H}), 4.91(\mathrm{~d}, J=3.7$ $\mathrm{Hz}, 1 \mathrm{H}), 4.33(\mathrm{~d}, J=3.7 \mathrm{~Hz}, 1 \mathrm{H}), 4.19(\mathrm{q}, J=7.1 \mathrm{~Hz}, 2 \mathrm{H}), 3.72(\mathrm{~s}, 3 \mathrm{H}), 1.22(\mathrm{t}, J=7.1 \mathrm{~Hz}, 3 \mathrm{H})$. ${ }^{13}$ C NMR (100 MHz, CDCl $) \delta 166.9,159.7,157.9,135.4,132.0,129.8,129.0,128.3,126.4$, 125.8, 119.0, 115.5, 86.9, 81.3, 62.0, 55.3, 14.1. HRMS (ESI) $\mathrm{m} / \mathrm{z}:[\mathrm{M}+\mathrm{Na}]^{+}$Calcd for

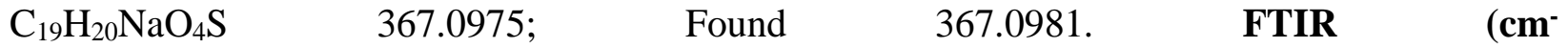
1) 2934, 1753, 1733, 1621, 1585, 1481, 1280, 1237, 1037.

Ethyl-2-((1-phenylvinyl)oxy)-2-(p-tolylthio)acetate (3au) and Ethyl-2-((1-phenylvinyl)oxy)-2-( $m$-tolylthio)acetate (3au')<smiles>C=C(OC(Sc1ccc(C)cc1)C(=O)OCC)C(OC(=O)c1ccccc1)Sc1cccc(C)c1</smiles>

Following the general procedure, treatment of 4 methyl-2-(trimethylsilyl)phenyl trifluoromethanesulfonate $\mathbf{2 j}(0.094 \mathrm{~g}, 0.3 \mathrm{mmol})$ with ethyl 2-((2-oxo-2-phenylethyl)thio)acetate 1a $(0.60 \mathrm{~g}, 0.25 \mathrm{mmol})$ in the presence of $\mathrm{KF}(0.035 \mathrm{~g}$, $0.6 \mathrm{mmol})$ and 18-crown-6 (0.158 g, $0.6 \mathrm{mmol})$ in

THF $(1.0 \mathrm{~mL})$ at $25{ }^{\circ} \mathrm{C}$ for $2 \mathrm{~h}$ followed by flash column chromatography (Pet.ether/EtOAc $=$ 97/03) of the crude reaction mixture using silica gel afforded ethyl-2-((1-phenylvinyl)oxy)-2-( $p$ tolylthio)acetate (3au) and ethyl-2-((1-phenylvinyl)oxy)-2-(m-tolylthio)acetate (3au') as a mixture of regioisomers in 1.1:1 ratio as a colorless oil $(0.065 \mathrm{~g}, 79 \%)$. 
$\boldsymbol{R}_{\mathbf{f}}($ Pet. ether $/$ EtOAc $=95 / 05): 0.43 ;{ }^{1} \mathbf{H}$ NMR (400 MHz, CDCl$)$ of Major Isomer; $\delta$ 7.67-7.63 (m, 2H), 7.47-7.45 (m, 1H), 7.39-7.33 (m, 4H), 7.24-7.13 (m, 2H), 5.71 (s, 1H), 4.91-4.89 (m, 1H), $4.33(\mathrm{~d}, J=3.3 \mathrm{~Hz}, 1 \mathrm{H}), 4.21-4.13(\mathrm{~m}, 2 \mathrm{H}), 2.32(\mathrm{~s}, 3 \mathrm{H}), 1.21(\mathrm{t}, J=7.1 \mathrm{~Hz}, 3 \mathrm{H})$. ${ }^{1} \mathbf{H}$ NMR (400 MHz, CDCl $)$ of Minor Isomer; $\delta$ 7.67-7.63 (m, 2H), 7.47-7.45 (m, 1H), 7.39-7.33 (m, 4H), 7.24$7.13(\mathrm{~m}, 2 \mathrm{H}), 5.68(\mathrm{~s}, 1 \mathrm{H}), 4.91-4.89(\mathrm{~m}, 1 \mathrm{H}), 4.33$ (d, J = 3.3 Hz, 1H), 4.21-4.13 (m, 2H), 2.35 $(\mathrm{s}, 3 \mathrm{H}), 1.21(\mathrm{t}, J=7.1 \mathrm{~Hz}, 3 \mathrm{H}) .{ }^{13} \mathbf{C}$ NMR (100 MHz, CDCl 3$)$ of Major Isomer; $\delta 167.0,157.9$, 138.8, 135.4, 135.0, 129.9, 129.85, 129.0, 128.9, 125.8, 87.0, 81.3, 61.9, 21.3, 14.1. ${ }^{\mathbf{1 3}} \mathbf{C}$ NMR (100 MHz, CDCl3) of Minor Isomer; $\delta$ 166.9, 157.9, 139.5, 135.4, 135.3, 131.6, 130.4, 129.0, 128.3, 126.9, 86.9, 81.4, 61.9, 21.4, 14.1. HRMS (ESI) m/z: $[\mathrm{M}+\mathrm{Na}]^{+}$Calcd for $\mathrm{C}_{19} \mathrm{H}_{18} \mathrm{NaO}_{3} \mathrm{~S}$ 351.1025; Found 351.1030. FTIR (cm-1) 2982, 1755, 1732, 1621, 1495, 1276, 1181, 1119.

\section{Ethyl-2-((4-chlorophenyl)thio)-2-((1-phenylvinyl)oxy)acetate and} Ethyl-2-((3-chlorophenyl)thio)-2-((1-phenylvinyl)oxy)acetate (3av and 3av')

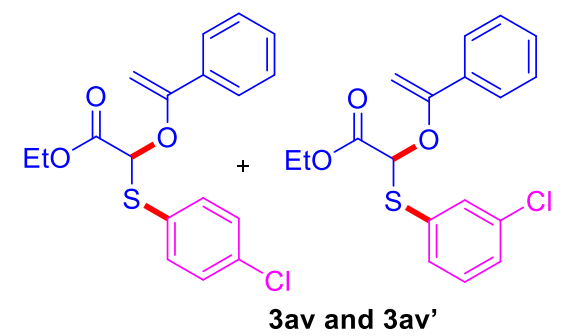

Following the general procedure, treatment of 4-chloro-2(trimethylsilyl)phenyl trifluoromethanesulfonate $\mathbf{2 k}(0.100 \mathrm{~g}$, $0.3 \mathrm{mmol})$ with ethyl 2-((2-oxo-2-phenylethyl)thio)acetate $\mathbf{1 a}$ $(0.060 \mathrm{~g}, 0.25 \mathrm{mmol})$ in the presence of $\mathrm{KF}(0.035 \mathrm{~g}, 0.6 \mathrm{mmol})$ and 18-crown-6 (0.158 g, $0.6 \mathrm{mmol})$ in THF $(1.0 \mathrm{~mL})$ at $25^{\circ} \mathrm{C}$ for $6 \mathrm{~h}$ followed by flash column chromatography

(Pet.ether/EtOAc $=97 / 03)$ of the crude reaction mixture using silica gel afforded ethyl-2-((4chlorophenyl)thio)-2-((1-phenylvinyl)oxy)acetate 3av and ethyl-2-((3-chlorophenyl)thio)-2-((1phenylvinyl)oxy)acetate 3av' as a colourless oil (0.074 g, 85\% yield).

$\boldsymbol{R}_{\mathbf{f}}\left(\right.$ Pet. ether $/$ EtOAc = 95/05): 0.47; ${ }^{1} \mathbf{H}$ NMR (400 MHz, CDCl $) \delta$ 7.67-7.64 (m, 3H), 7.53-7.50 (m, 2H), 7.42-7.35 (m, 3H), $5.72(\mathrm{~s}, 1 \mathrm{H}), 4.95-4.94(\mathrm{~m}, 1 \mathrm{H}), 4.37$ (d, J = 3.7 Hz, 1H), 4.25-4.16 $(\mathrm{m}, 2 \mathrm{H}), 1.24(\mathrm{t}, J=7.1 \mathrm{~Hz}, 3 \mathrm{H}) .{ }^{13} \mathrm{C}$ NMR (100 MHz, CDCl3) $\delta$ 166.6, 157.8, 136.1, 135.2, $134.3,132.6,129.3,129.1,128.4,125.8,87.1,80.8,62.1,14.2$. Representative peaks of the minor isomer ${ }^{1} \mathbf{H}$ NMR (400 MHz, CDCl3) $\delta$ 7.62-7.61 (m), 7.49-7.46 (m), 7.33-7.28 (m). 5.74 (s). ${ }^{13}$ C NMR (100 MHz, CDCl3) $\delta$ 157.83, 135.7, 135.21, 134.5, 132.5, 130.1, 129.30, 129.0, 125.79, 87.3, 80.77, 62.12, 14.1 HRMS (ESI) m/z: [M+Na] ${ }^{+}$Calcd for $\mathrm{C}_{18} \mathrm{H}_{17} \mathrm{ClNaO}_{3} \mathrm{~S} 371.0479$; Found 371.0487. FTIR (cm-1) 2983, 2365, 1754, 1732, 1622, 1472, 1276, 1182, 1120. 
Ethyl-2-((4-fluorophenyl)thio)-2-((1-phenylvinyl)oxy)acetate and Ethyl -2-((3-fluorophenyl)thio)-2-((1-phenylvinyl)oxy)acetate (3aw and 3aw')

Following the general procedure, treatment of 5-fluoro-2-(trimethylsilyl)phenyl trifluoromethanesulfonate $2 \mathbf{2}(0.095 \mathrm{~g}, 0.3 \mathrm{mmol})$ with ethyl 2-((2-oxo-2-phenylethyl)thio)acetate $1 \mathrm{a}(0.060 \mathrm{~g}, 0.25 \mathrm{mmol})$ in the presence of $\mathrm{KF}(0.035 \mathrm{~g}, 0.6 \mathrm{mmol})$ and 18 -crown-6 $(0.158 \mathrm{~g}, 0.6$

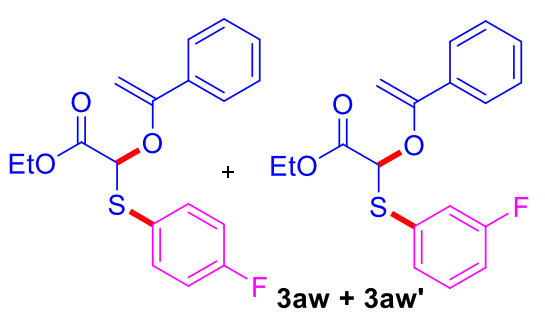
$\mathrm{mmol})$ in THF $(1.0 \mathrm{~mL})$ at $25^{\circ} \mathrm{C}$ for $6 \mathrm{~h}$ followed by flash column chromatography (Pet.ether/EtOAc $=97 / 03)$ of the crude reaction mixture using silica gel afforded Ethyl-2-((4fluorophenyl)thio)-2-((1-phenylvinyl)oxy)acetate 3aw and ethyl -2-((3-fluorophenyl)thio)-2-((1-phenylvinyl)oxy)acetate 3aw' as a colourless oil $(0.077 \mathrm{~g}, 93 \%$ yield $)$.

$\boldsymbol{R}_{\mathbf{f}}($ Pet. ether $/ \mathrm{EtOAc}=95 / 05)$ : 0.47; ${ }^{1} \mathbf{H}$ NMR (400 MHz, CDCl3) $\delta$ 7.65-7.60 (m, 2H), 7.57-7.52 (m, 2H), 7.39-7.33 (m, 3H), 7.08-6.99 (m, 2H), $5.66(\mathrm{~s}, 1 \mathrm{H}), 4.92-4.91(\mathrm{~m}, 1 \mathrm{H}), 4.34(\mathrm{~d}, J=3.7$

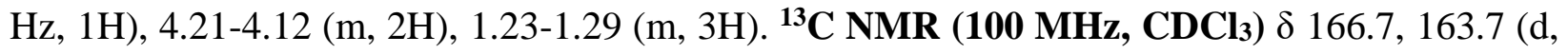
$J=249.1 \mathrm{~Hz}), 157.8,137.4,135.3,130.3$ (d, $J=8.0 \mathrm{~Hz}), 129.8,129.1,128.4,125.8,125.4,121.0$ $(\mathrm{d}, J=22.2 \mathrm{~Hz}), 116.2(\mathrm{~d}, J=22.0 \mathrm{~Hz}), 87.0,80.9,62.0,14.2$ (Representative peaks of the minor isomer ${ }^{1} \mathrm{H}$ NMR (400 MHz, $\left.\mathbf{C D C l}_{3}\right) \delta$ 7.31-7.27 (m), $5.73(\mathrm{~s}){ }^{13} \mathrm{C}$ NMR (100 MHz, CDCl 3$) \delta$ 166.6, 162.4 (d, $J=248.0 \mathrm{~Hz}), 157.9$, 137.36, 135.2, 129.9, 129.12, 129.4, 125.77, 125.3, 116.1(d, $J=21.0 \mathrm{~Hz}$ ), 87.2, 80.91, 62.1, 14.1. HRMS (ESI) m/z: $[\mathrm{M}+\mathrm{Na}]^{+}$Calcd for $\mathrm{C}_{18} \mathrm{H}_{17} \mathrm{FNaO}_{3} \mathrm{~S}$ 355.0775; Found 355.0782. FTIR (cm-1) 3437, 2984, 1730, 1681, 1589, 1490, 1269, 1071, 836.

\section{1-Phenyl-2-(phenylthio)-2-((1-phenylvinyl)oxy)ethan-1-one (10a)}

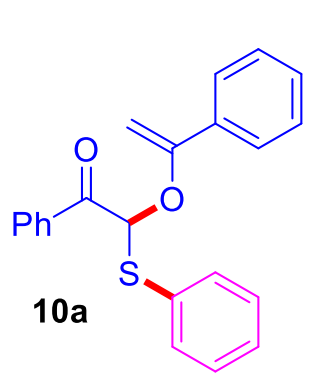

Following the general procedure, treatment of 2-(trimethylsilyl) phenyltrifluoromethanesulfonate $\mathbf{2 a}(0.179 \mathrm{~g}, 146 \mu \mathrm{L}, 0.6 \mathrm{mmol})$ with 2,2'thiobis(1-phenylethan-1-one $)^{9} \mathbf{9 a}(0.135 \mathrm{~g}, 0.5 \mathrm{mmol})$ in the presence of $\mathrm{KF}$ $(0.070 \mathrm{~g}, 1.2 \mathrm{mmol})$ and 18-crown-6 (0.317 g, $1.2 \mathrm{mmol})$ in THF $(2.0 \mathrm{~mL})$ at $25{ }^{\circ} \mathrm{C}$ for $1 \mathrm{~h}$ followed by flash column chromatography (Pet.ether/EtOAc $=$ 97/03) of the crude reaction mixture using silica gel afforded 1-phenyl-2(phenylthio)-2-((1-phenylvinyl)oxy)ethan-1-one 10a as a colourless oil (0.164 g, 95\% yield).

\footnotetext{
${ }^{9}$ Eller, C.; Kehr, G.; Daniliuc, C. G.; Stephan, D. W.; Erker, G. Chem. Commun. 2015, 51, 7226.
} 
$\boldsymbol{R}_{\mathbf{f}}\left(\right.$ Pet. ether $/$ EtOAc = 95/05): 0.52; ${ }^{1} \mathbf{H}$ NMR (400 MHz, $\left.\mathbf{C D C l}_{3}\right) \delta$ 8.15-8.13 (m, 2H), 7.73-7.71 $(\mathrm{m}, 2 \mathrm{H}), 7.64-7.60(\mathrm{~m}, 1 \mathrm{H}), 7.54-7.43(\mathrm{~m}, 4 \mathrm{H}), 7.42-7.31(\mathrm{~m}, 6 \mathrm{H}) 6.50(\mathrm{~s}, 1 \mathrm{H}), 4.95(\mathrm{~d}, J=3.7$ Hz, 1H), 4.42 (d, $J=3.7$ Hz, 1H). ${ }^{13}$ C NMR (100 MHz, CDCl3) $\delta$ 190.1, 157.9, 135.3, 134.0, 133.99, 133.7, 131.1, 129.2, 129.1, 129.0, 128.95, 128.7, 128.3, 125.7, 87.3, 85.2. HRMS (ESI) m/z: $[\mathrm{M}+\mathrm{Na}]^{+}$Calcd for $\mathrm{C}_{22} \mathrm{H}_{18} \mathrm{NaO}_{2} \mathrm{~S}$ 369.0920; Found 369.0923. FTIR (cm-1) 2851, 1695, 1599, 1444, 1275, 1118, 979.

\section{Product Functionalization}

a) Procedure for Synthesis of Compound 11 and 12<smiles>[R]c1ccc(C(=C)OC(C(=O)OCC)C(=O)c2ccccc2)cc1</smiles><smiles>[R]c1ccc(C(=C)OC(C(=O)NC)c2ccccc2)cc1</smiles>

To stirred solution of enol ether 3 (1.0 equiv) in ethanol $(0.5 \mathrm{~mL})$ was added $33 \%$ ethanol solution of methylamine (3.0 equiv) under $\mathrm{N}_{2}$ atmosphere at room temperature. The reaction mixture was stirred for $30 \mathrm{~h}$ at room temperature $\left(25^{\circ} \mathrm{C}\right)$ followed by evaporation of solvent under reduced pressure and the crude product was purified by column chromatography using mixture of pet. ether/ethyl acetate as an eluent to afford the corresponding amide derivatives.

\section{N-Methyl-2-(phenylthio)-2-((1-phenylvinyl)oxy)acetamide (11)}

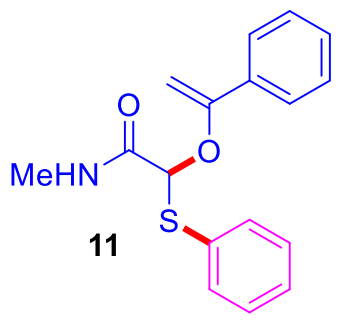

Following the general procedure, to stirred solution of ethyl 2-(phenylthio)2-((1-phenylvinyl)oxy)acetate $3 \mathbf{a}(0.063 \mathrm{~g}, 0.20 \mathrm{mmol}, 1.0$ equiv) in ethanol $(0.5 \mathrm{~mL})$ was added $33 \%$ ethanol solution of methylamine $(0.075 \mathrm{~mL}, 0.60$ mmol, 3.0 equiv) under $\mathrm{N}_{2}$ atmosphere at room temperature. The reaction mixture was stirred for $30 \mathrm{~h}$ at room temperature $\left(25^{\circ} \mathrm{C}\right)$ followed by evaporation under reduced pressure and the crude product was purified by column chromatography using mixture of pet. ether/ethyl acetate (80/20) as an eluent to afford the $\mathbf{1 1}$ as a white solid (0.043 $\mathrm{mg}, 72 \%)$

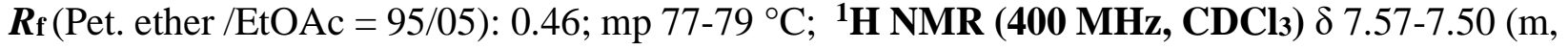
4H), 7.37-7.28 (m, 6H), $6.01(\mathrm{bs}, 1 \mathrm{H}), 5.68(\mathrm{~s}, 1 \mathrm{H}), 4.95(\mathrm{~d}, J=3.7 \mathrm{~Hz}, 1 \mathrm{H}), 4.46(\mathrm{~d}, J=3.7 \mathrm{~Hz}$, 1H), $2.66(\mathrm{~d}, J=5.0 \mathrm{~Hz}, 3 \mathrm{H}) .{ }^{13} \mathbf{C}$ NMR (100 MHz, CDCl$) \delta 167.2,157.1,135.4,135.39,130.4$, 129.3, 129.2, 129.0, 128.5, 125.7, 88.8, 83.5, 26.2. HRMS (ESI) m/z: [M+Na] ${ }^{+}$Calcd for 
$\mathrm{C}_{17} \mathrm{H}_{17} \mathrm{NNaO}_{2} \mathrm{~S}$ 322.0872; Found 322.0877 . FTIR (cm-1) 3448, 2923, 2852, 1743, 1670, 1535, 1408, 1268, 1114, 1026.

\section{2-((1-(4-Bromophenyl)vinyl)oxy)- $N$-methyl-2-(phenylthio)acetamide (12)}

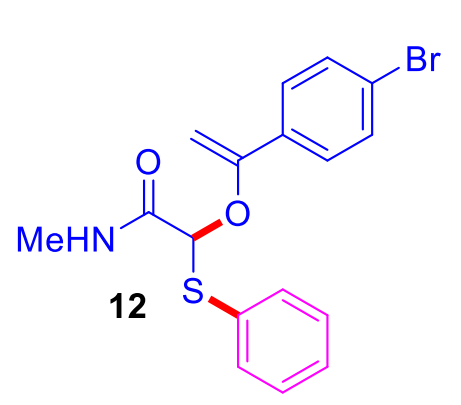

Following the general procedure, to stirred solution of ethyl-2-((1(4-bromophenyl)vinyl)oxy)-2-(phenylthio)acetate 3d (0.088 g, 0.22 mmol, 1.0 equiv) in ethanol $(0.5 \mathrm{~mL})$ was added $33 \%$ ethanol solution of methylamine ( $0.084 \mu \mathrm{L}, 0.67 \mathrm{mmol}, 3.0$ equiv) under $\mathrm{N}_{2}$ atmosphere at room temperature. The reaction mixture was stirred for $30 \mathrm{~h}$ at room temperature $\left(25^{\circ} \mathrm{C}\right)$ followed by evaporation under reduced pressure and the crude product was purified by column chromatography using mixture of pet. ether/ethyl acetate (80/20) as an eluent to afford the 2-((1-(4-bromophenyl)vinyl)oxy)- $N$ methyl-2-(phenylthio)acetamide $\mathbf{1 2}$ as a sticky oil $(0.052 \mathrm{mg}, 61 \%)$.

$\boldsymbol{R}_{\mathbf{f}}\left(\mathrm{Pet}\right.$. ether /EtOAc = 70/30): 0.32; ${ }^{\mathbf{1}} \mathbf{H}$ NMR $\left(\mathbf{4 0 0} \mathbf{~ M H z}, \mathbf{C D C l}_{\mathbf{3}}\right) \delta$ 7.53-7.47 (m, 4H), 7.38-7.29 (m, 5H), 5.96 (bs, 1H), 5.66 (s, 1H), $4.94(\mathrm{~d}, J=3.6 \mathrm{~Hz}, 1 \mathrm{H}), 4.47$ (d, $J=3.6 \mathrm{~Hz}, 1 \mathrm{H}), 2.68$ (d, $J$ $=4.8 \mathrm{~Hz}, 3 \mathrm{H}) .{ }^{13} \mathbf{C}$ NMR (100 MHz, $\left.\mathbf{C D C l}_{3}\right) \delta 167.0,156.2,135.3,134.3,131.7,130.2,129.4$, 129.1, 127.2, 123.3, 89.2, 83.5, 26.2. HRMS (ESI) m/z: $[\mathrm{M}+\mathrm{Na}]^{+}$Calcd for $\mathrm{C}_{17} \mathrm{H}_{16} \mathrm{BrNNaO}_{2} \mathrm{~S}$ 399.9977; Found 399.9980. FTIR (cm-1) 3306, 1667, 1621, 1529, 1483, 1263, 1109, 1009, 943

\section{b) General Procedure for the Synthesis of 1,3-Dioxolan-4-one Derivatives}<smiles>[R]C(=C)OC(C(=O)OCC)c1ccccc1</smiles>

$$
\underset{25^{\circ} \mathrm{C} \text {, overnight }}{\stackrel{\text { THF: } \mathrm{H}_{2} \mathrm{O}(2: 1)}{\longrightarrow}}
$$

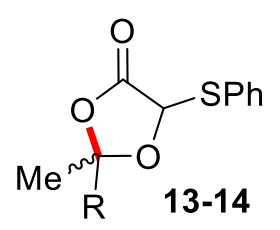

13-14

To stirred solution of 3 (1.0 equiv) in THF: $\mathrm{H}_{2} \mathrm{O}$ (2:1) was added $\mathrm{LiOH}$ (3.0 equiv) at 25 ${ }^{\circ} \mathrm{C}$. Reaction mixture was allowed to stir for overnight at $25^{\circ} \mathrm{C}$. After completion of the reaction, monitored by TLC, the reaction was diluted with $\mathrm{H}_{2} \mathrm{O}(10 \mathrm{~mL})$ and extracted with $\mathrm{Et}_{2} \mathrm{O}(10 \mathrm{~mL} \mathrm{x}$ 3). The aqueous phase was acidified using $1 \mathrm{M} \mathrm{HCl}$ solution $(\mathrm{pH}=6-7)$ and extracted with EtOAc $(10 \mathrm{~mL} \times 3)$. At this stage, we clearly observed the hydrolysis product (acid) from the TLC. Then combined organic layers were dried $\left(\mathrm{Na}_{2} \mathrm{SO}_{4}\right)$, filtered and concentrated under reduced pressure at $50{ }^{\circ} \mathrm{C}$ (cyclized product was observed upon organic layer concentration under reduced pressure at 
$50{ }^{\circ} \mathrm{C}$ ). The crude product was purified by column chromatography using mixture of pet. ether/ethyl acetate (97/03) as an eluent to afford the 13-14.

\section{2-Methyl-2-phenyl-5-(phenylthio)-1,3-dioxolan-4-one (13)}<smiles>C[C@]1(c2ccccc2)OC(=O)C(c2ccccc2)O1</smiles>

13

Following the general procedure, to stirred solution of ethyl 2-(phenylthio)2-((1-phenylvinyl)oxy)acetate 3a ( $0.2 \mathrm{~g}, 0.64 \mathrm{mmol}, 1.0$ equiv) in THF (4.0

$\mathrm{mL})$ :water $(2.0 \mathrm{~mL})$ was added $\mathrm{LiOH}(0.080 \mathrm{~g}, 1.9 \mathrm{mmol}, 3.0$ equiv) at room temperature. Reaction mixture was allowed to stir for overnight at room temperature. After completion of the reaction, monitored by TLC, the reaction was diluted with water $(10 \mathrm{~mL})$ and extracted with $\mathrm{Et}_{2} \mathrm{O}(10 \mathrm{~mL} \times 3)$. The aqueous phase was acidified using $1 \mathrm{M} \mathrm{HCl}$ solution $(\mathrm{pH}=6-7)$ and extracted with EtOAc $(10 \mathrm{~mL} \times 3)$. At this stage we clearly observed the hydrolysis product (acid) from the TLC. Then combined organic layers were dried $\left(\mathrm{Na}_{2} \mathrm{SO}_{4}\right)$, filtered and concentrated under reduced pressure at $50{ }^{\circ} \mathrm{C}$ (cyclized product was observed upon organic layer concentration under reduced pressure at $50{ }^{\circ} \mathrm{C}$ ). The crude product was purified by column chromatography using mixture of pet. ether/ethyl acetate $(97 / 03)$ as an eluent to afford the $\mathbf{1 3}$ as a colorless oil $(0.141 \mathrm{mg}, 77 \%$ yield; diastereoselectivity determined by crude ${ }^{1} \mathrm{H}$ NMR is $>20: 1$ )

$\boldsymbol{R}_{\mathbf{f}}\left(\right.$ Pet. ether /EtOAc $=$ 95/05): 0.46; ${ }^{\mathbf{1}} \mathbf{H}$ NMR $\left(\mathbf{4 0 0} \mathbf{~ M H z}, \mathbf{C D C l}_{3}\right) \delta$ 7.45-7.30 (m, 7H), 7.27-7.17 (m, 3H), 5.83 (s, 1H), 1.83 (s, 3H). ${ }^{13} \mathbf{C}$ NMR (100 MHz, CDCl $) \delta$ 168.6, 140.1, 133.9, 131.0, 129.2, 129.2, 128.9, 128.4, 125.0, 111.5, 81.9, 29.2. HRMS (ESI) $\mathrm{m} / \mathrm{z}:[\mathrm{M}+\mathrm{H}]^{+}$Calcd for $\mathrm{C}_{16} \mathrm{H}_{15} \mathrm{O}_{3} \mathrm{~S}$ 287.0736; Found 287.1469. FTIR (cm-1) 3062, 1799, 1444, 1239, 1096, 1021, 949

\section{2-Methyl-2-(naphthalen-1-yl)-5-(phenylthio)-1,3-dioxolan-4-one (14)}

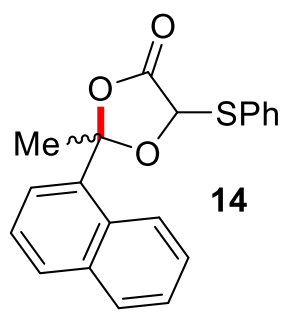
Following general procedure, to stirred solution of ethyl 2-((1-(naphthalen-1yl)vinyl)oxy)-2-(phenylthio)acetate $3 r(0.070 \mathrm{~g}, 0.19 \mathrm{mmol}, 1.0$ equiv) in THF $(1.2 \mathrm{~mL})$ :water $(0.6 \mathrm{~mL})$ was added $\mathrm{LiOH}(0.024 \mathrm{~g}, 0.58 \mathrm{mmol}, 3.0$ equiv) at room temperature. Reaction mixture was allowed to stir for overnight at room temperature. After completion of the reaction, monitored by TLC, the reaction was diluted with water $(3 \mathrm{~mL})$ and extracted with $\mathrm{Et}_{2} \mathrm{O}(3 \mathrm{~mL} \times 3)$. The aqueous phase was acidified using $1 \mathrm{M} \mathrm{HCl}$ solution $(\mathrm{pH}=6-7)$ and extracted with EtOAc (3 $\mathrm{mL} \times 3)$. At this stage we clearly observed the hydrolysis product (acid) from the TLC. Then combined organic layers 
were dried $\left(\mathrm{Na}_{2} \mathrm{SO}_{4}\right)$, filtered and concentrated under reduced pressure at $50{ }^{\circ} \mathrm{C}$ (cyclized product was observed upon organic layer concentration under reduced pressure at $50{ }^{\circ} \mathrm{C}$ ). The crude product was purified by column chromatography using mixture of pet. ether/ethyl acetate (97/03) as an eluent to afford the 2-methyl-2-(naphthalen-1-yl)-5-(phenylthio)-1,3-dioxolan-4-one $\mathbf{1 4}$ as a colorless oil ( $0.036 \mathrm{mg}, 55 \%$ yield; diastereoselectivity determined by crude ${ }^{1} \mathrm{H}$ NMR is $\left.>20: 1\right)$ $\boldsymbol{R}_{\mathbf{f}}\left(\right.$ Pet. ether $/$ EtOAc $=$ 95/05): 0.46; ${ }^{1} \mathbf{H}$ NMR $\left(\mathbf{4 0 0} \mathbf{~ M H z}, \mathbf{C D C l}_{3}\right) \delta 8.40(\mathrm{~d}, J=8.3 \mathrm{~Hz}, 1 \mathrm{H})$, 7.91-7.85 (m, 2H), 7.61-7.52 (m, 3H), 7.40-7.36 (m, 1H), 7.23-7.15 (m, 3H), 7.06-7.03 (m, 2H), $5.93(\mathrm{~s}, 1 \mathrm{H}), 2.08(\mathrm{~m}, 3 \mathrm{H}){ }^{13} \mathbf{C}$ NMR (100 MHz, CDCl $) \delta$ 168.5, 136.1, 134.4, 134.2, 130.8, 130.3, 129.5, 129.04, 129.0, 128.95, 126.5, 125.9, 125.8, 124.9, 123.5, 112.7, 82.1, 29.3 HRMS (ESI) $\mathrm{m} / \mathrm{z}: \quad[\mathrm{M}+\mathrm{H}]^{+}$Calcd for $\mathrm{C}_{20} \mathrm{H}_{17} \mathrm{O}_{3} \mathrm{~S}$ 337.0893; Found 337.0896. FTIR (cm1) $3054,2367,1801,1512,1236,1202,1094,1020,946$. 


\section{9. ${ }^{1} \mathrm{H}$ and ${ }^{13} \mathrm{C}$ NMR Spectra of $\beta$-Keto Thioetherss}

Ethyl 2-((2-oxo-2-phenylethyl)thio)acetate (1a $)^{4}$
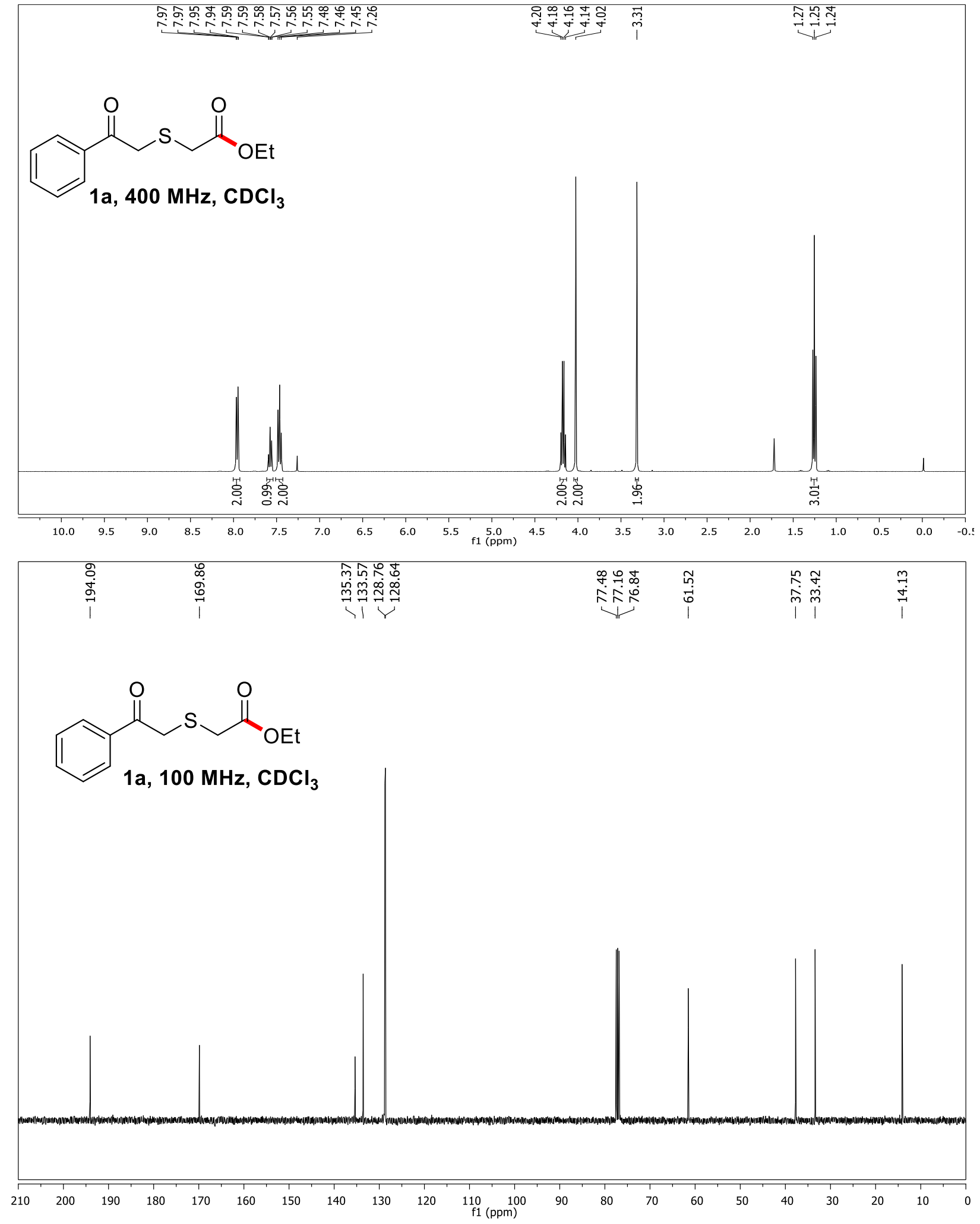
Ethyl 2-((2-(4-methoxyphenyl)-2-oxoethyl)thio)acetate(1b)
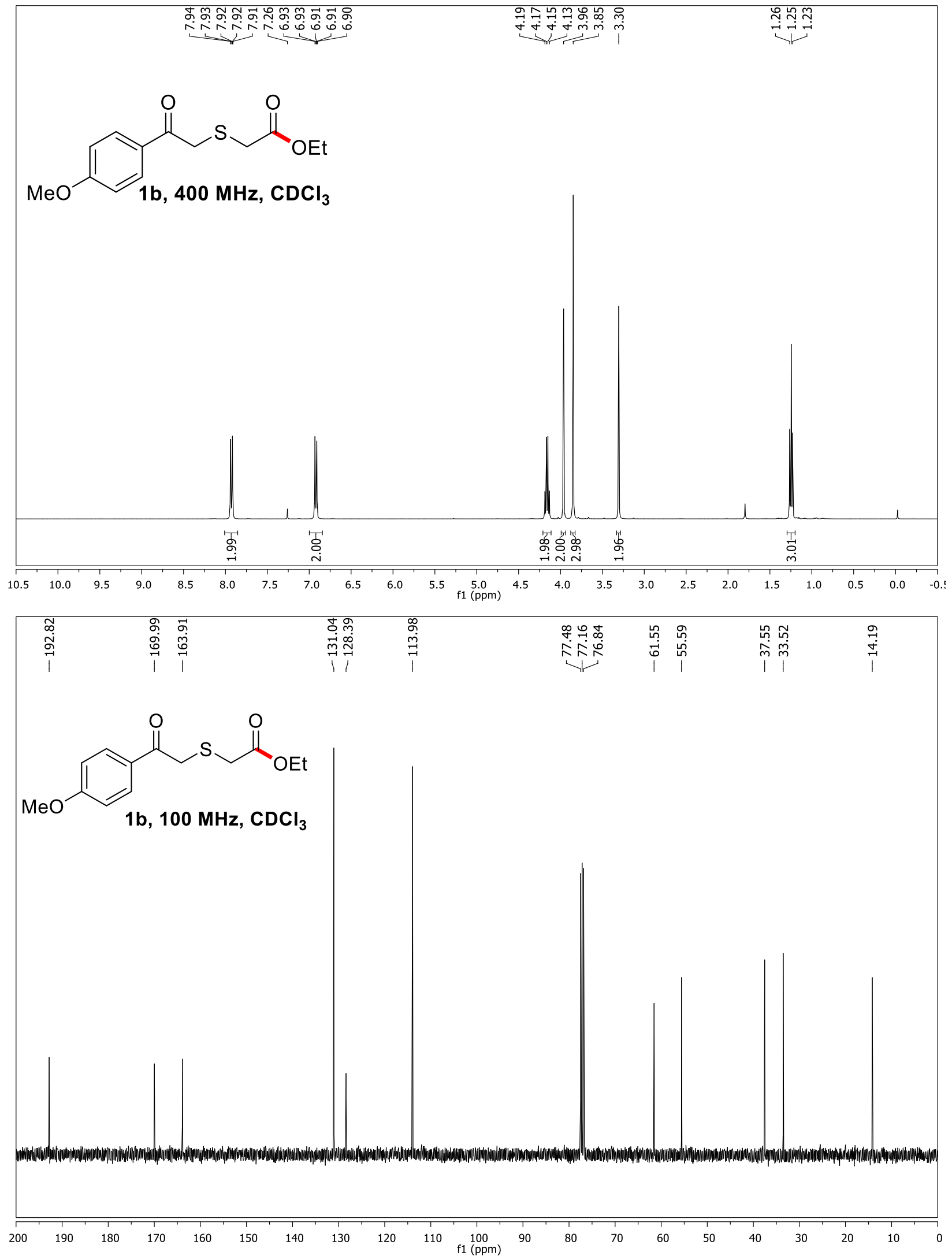
Ethyl 2-((2-oxo-2-(p-tolyl)ethyl)thio)acetate (1c)
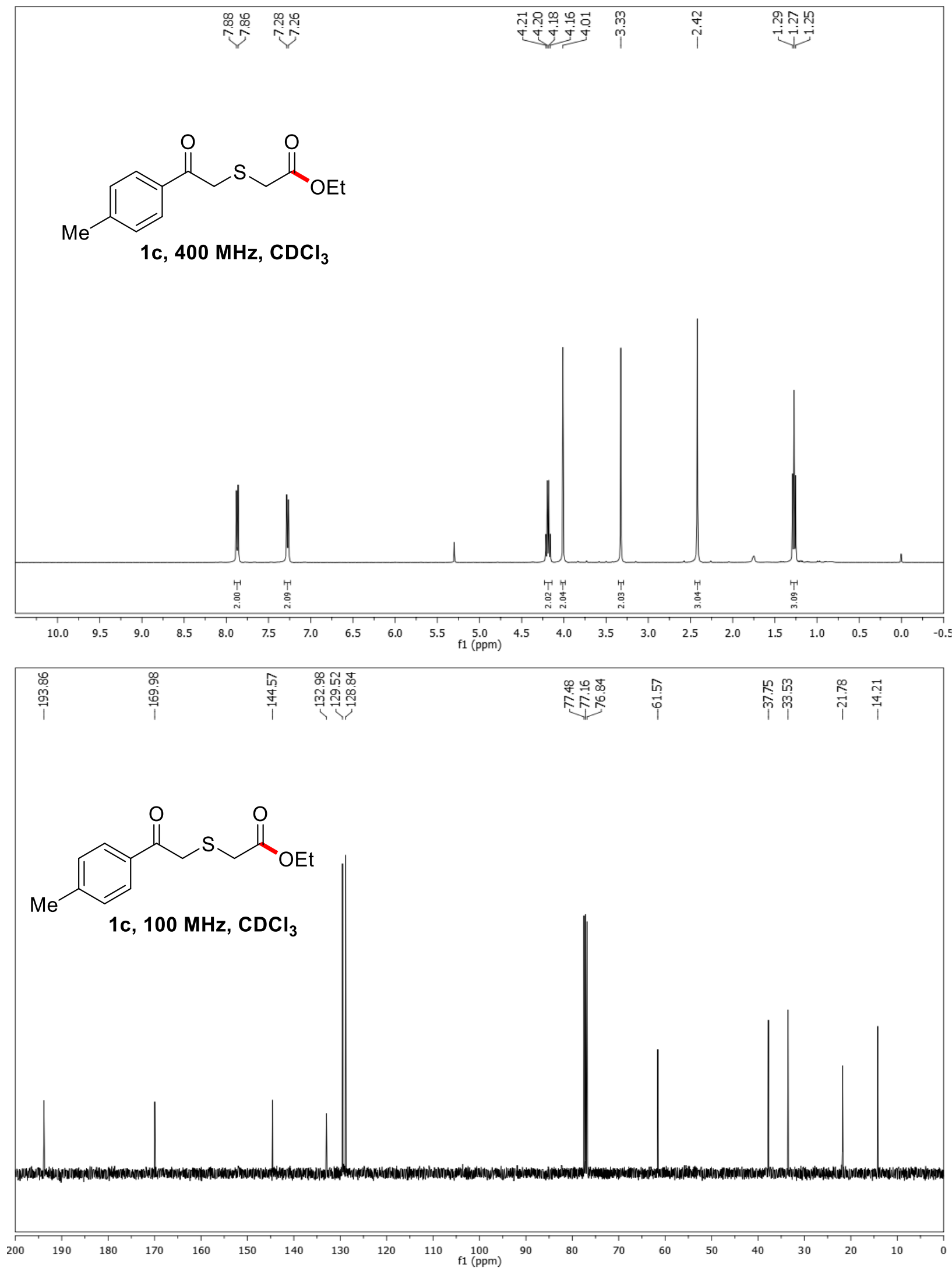
Ethyl 2-((2-(4-bromophenyl)-2-oxoethyl)thio)acetate (1d)
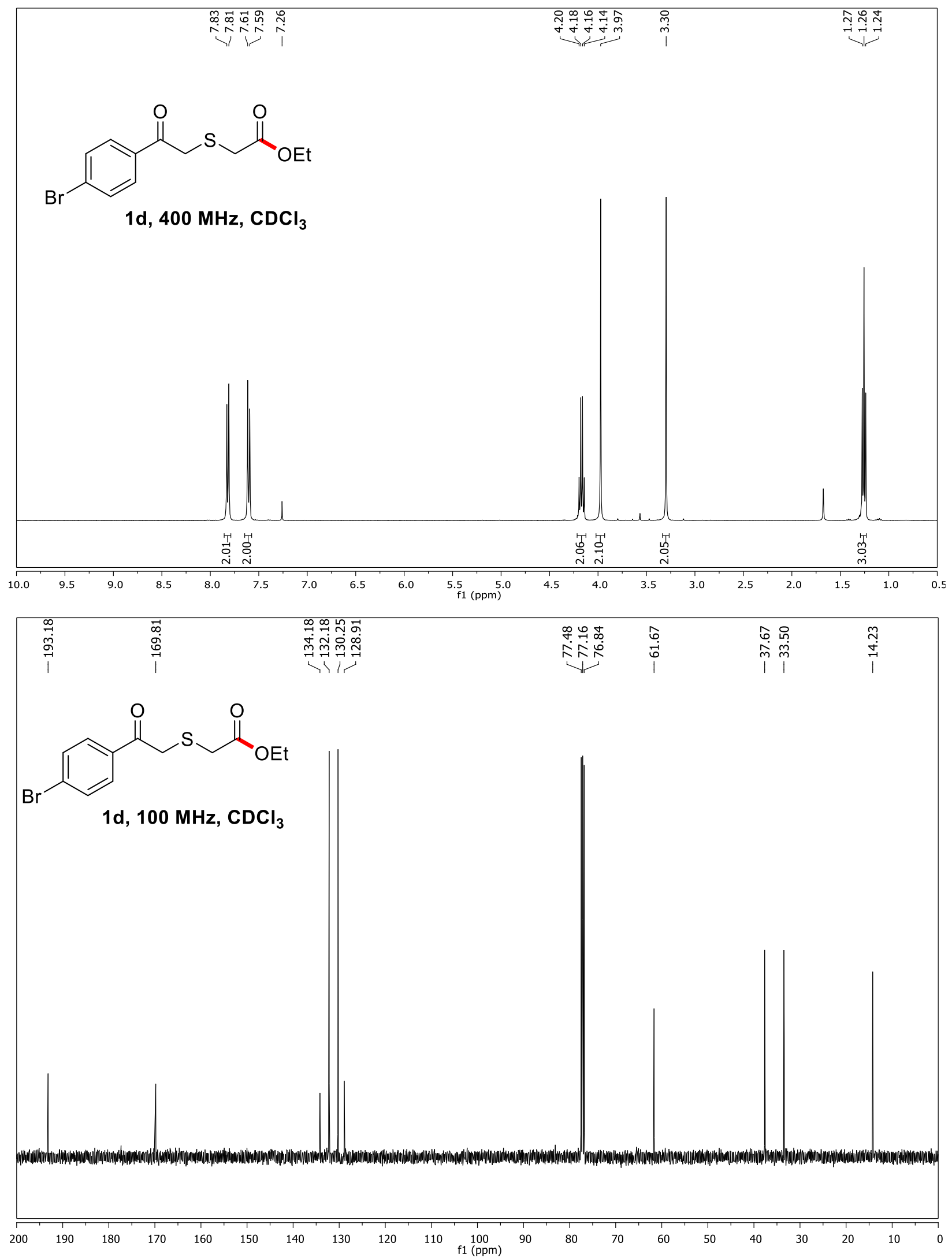
Ethyl 2-((2-(4-chlorophenyl)-2-oxoethyl)thio)acetate (1e)
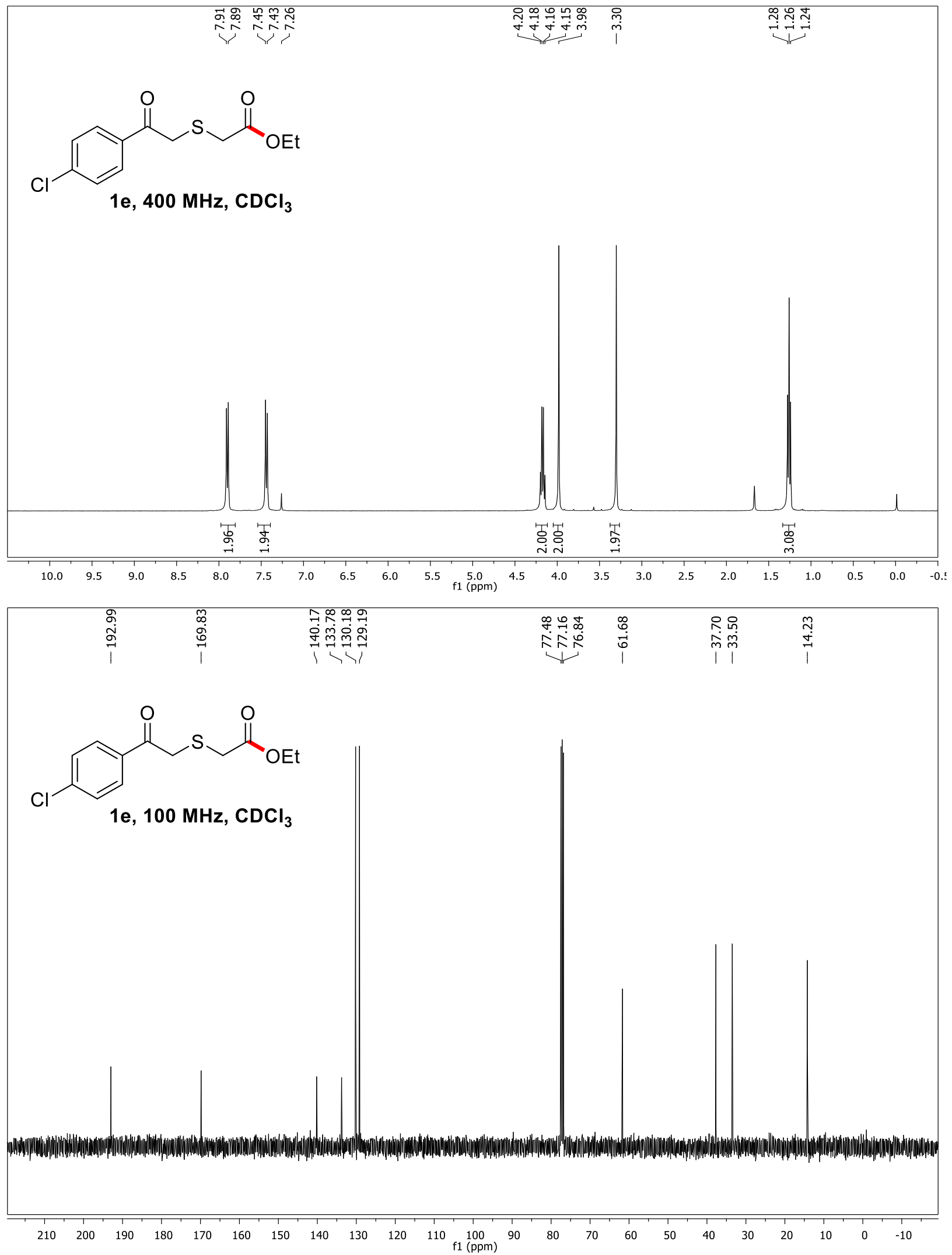
Ethyl 2-((2-(4-fluorophenyl)-2-oxoethyl)thio)acetate (1f)
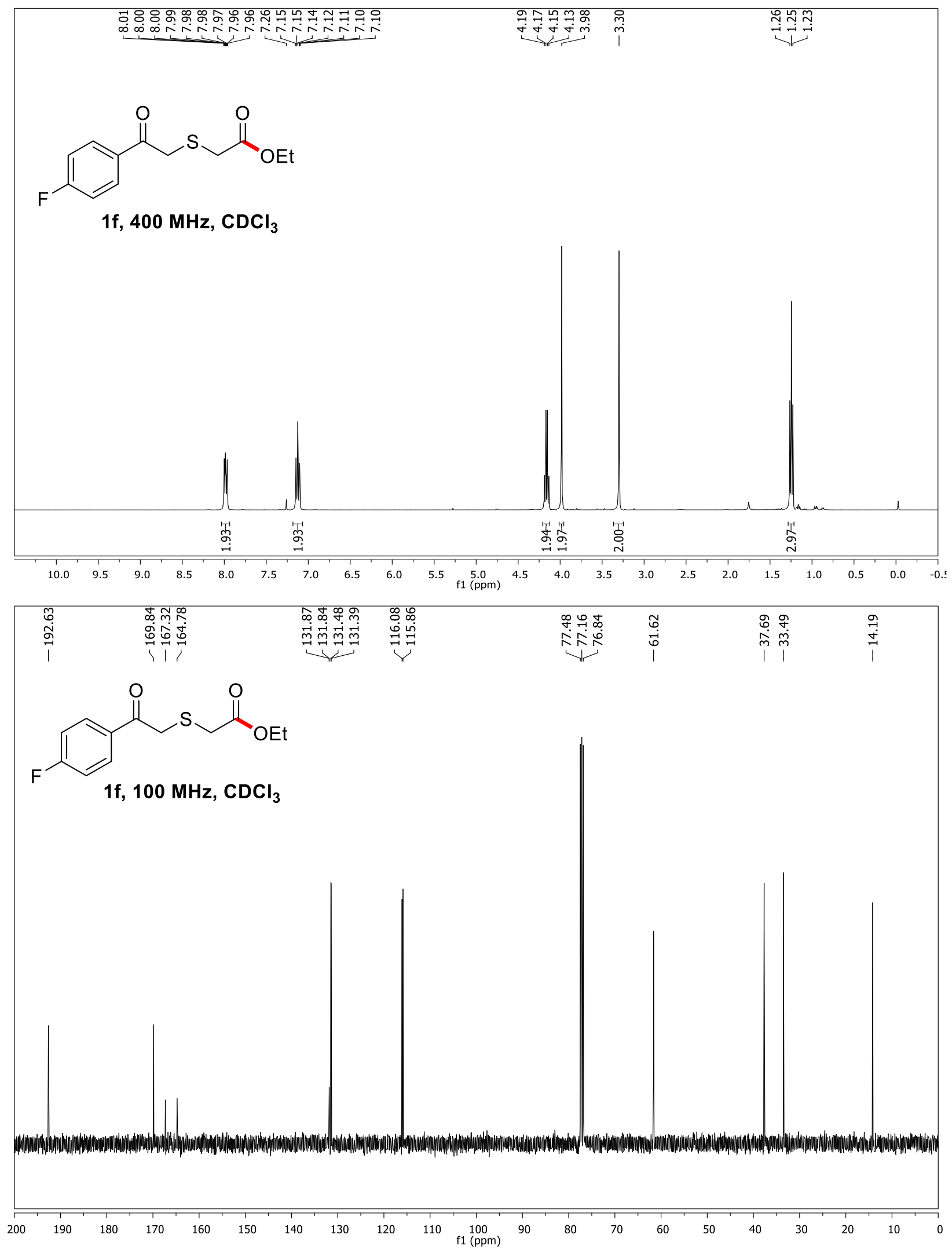
Ethyl 2-((2-(4-cyanophenyl)-2-oxoethyl)thio)acetate (1g)
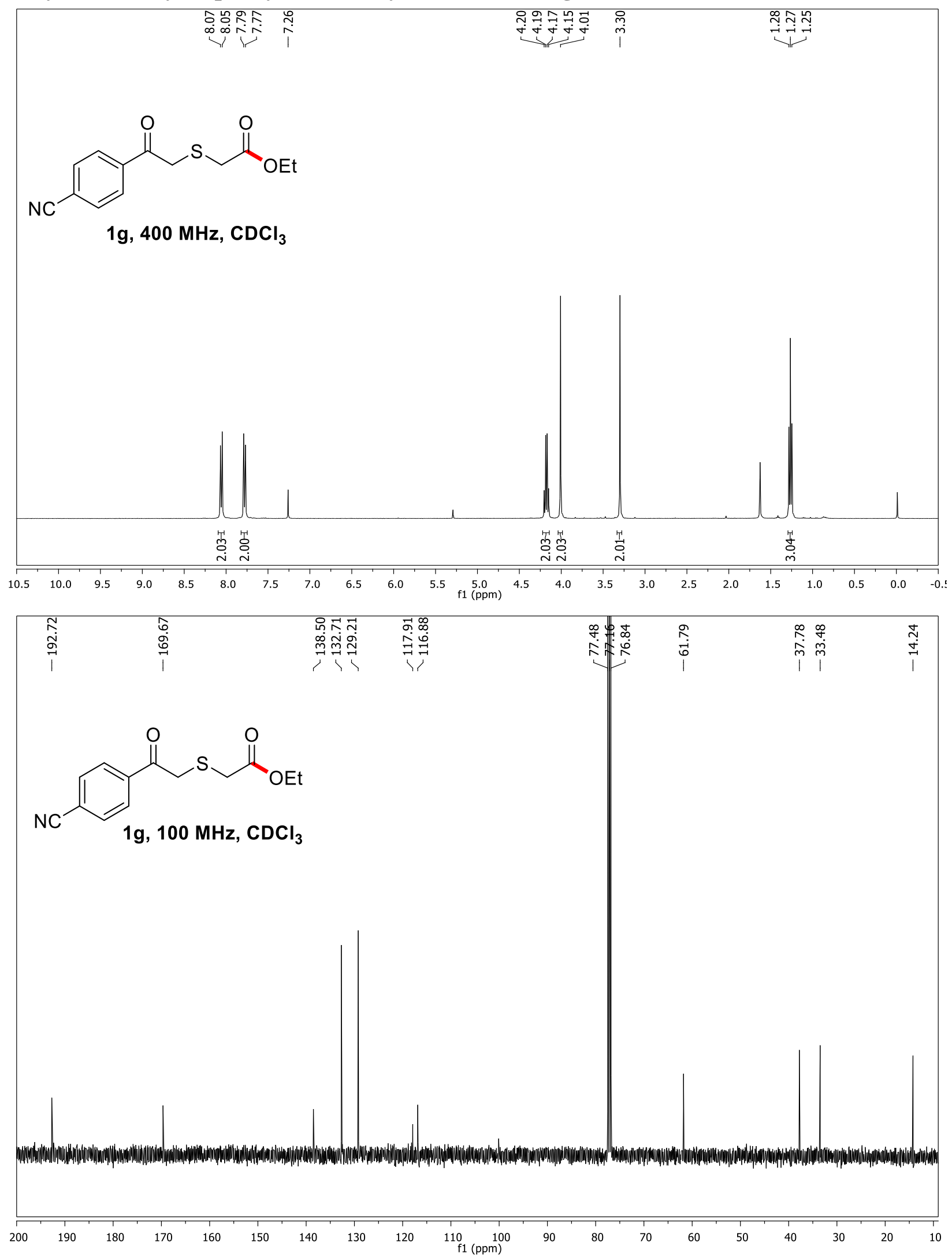
Ethyl 2-((2-(4-nitrophenyl)-2-oxoethyl)thio)acetate (1h)
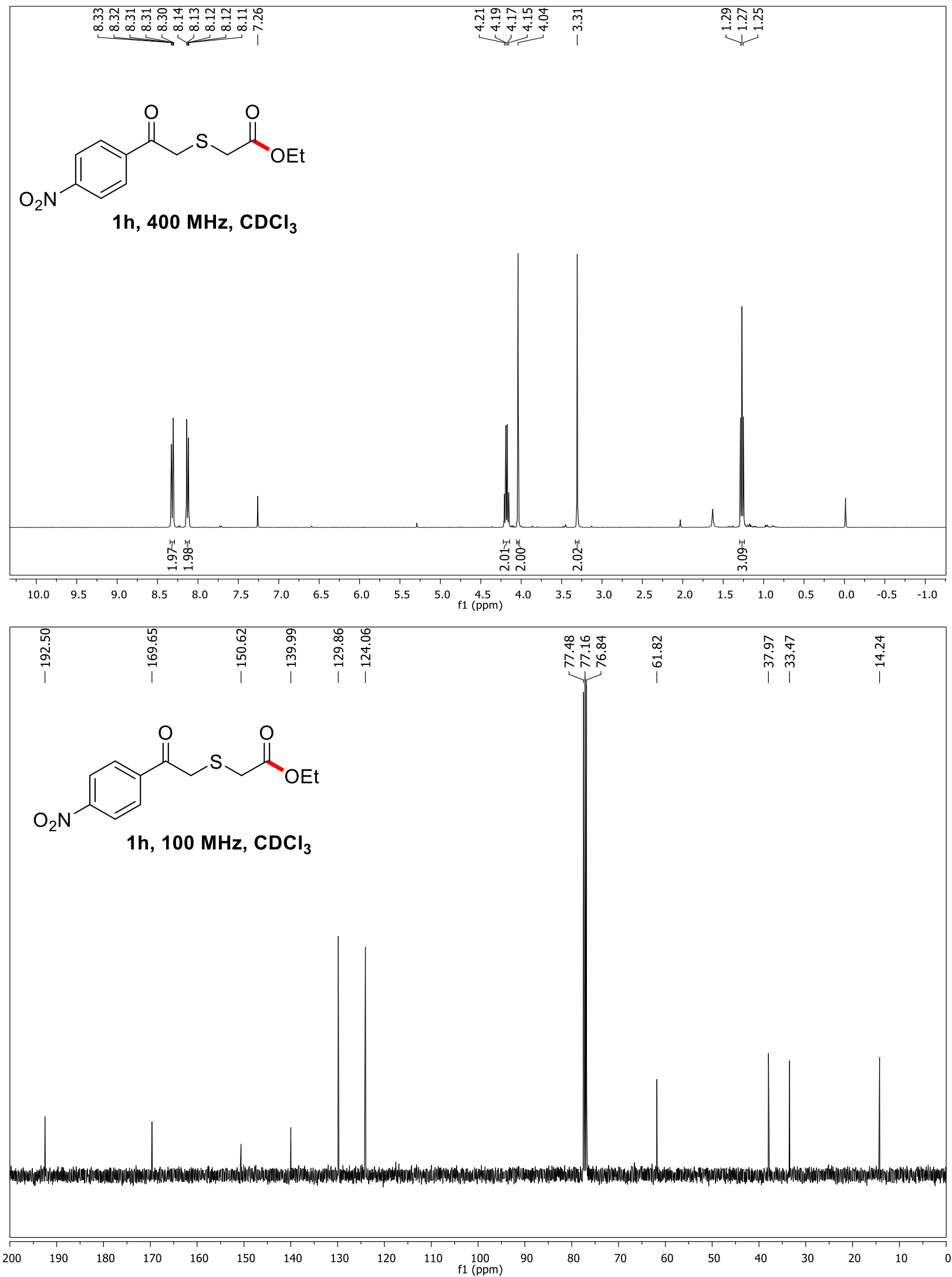
ethyl 2-((2-(3-bromophenyl)-2-oxoethyl)thio)acetate (1i)
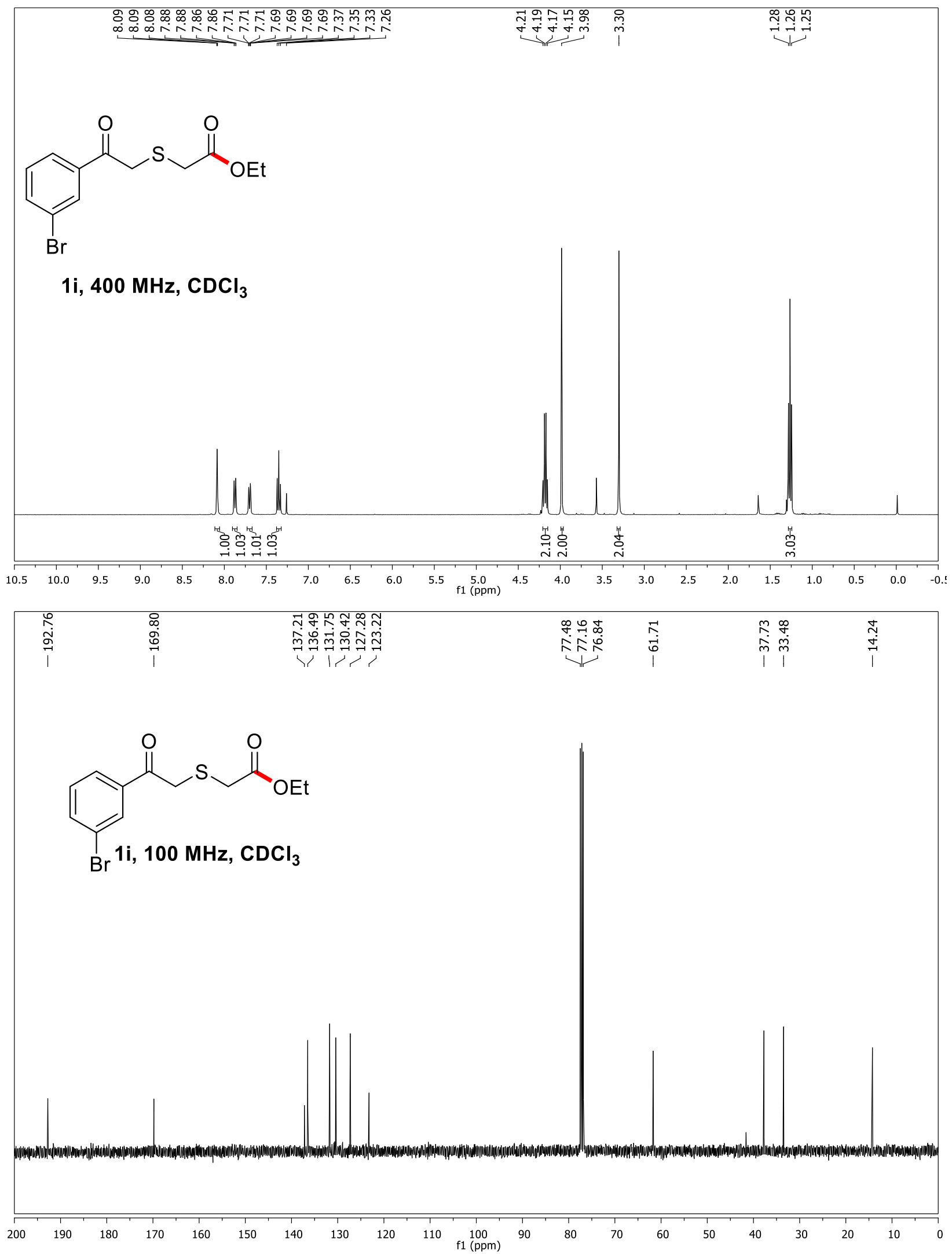
Ethyl 2-((2-(3-chlorophenyl)-2-oxoethyl)thio)acetate (1j)
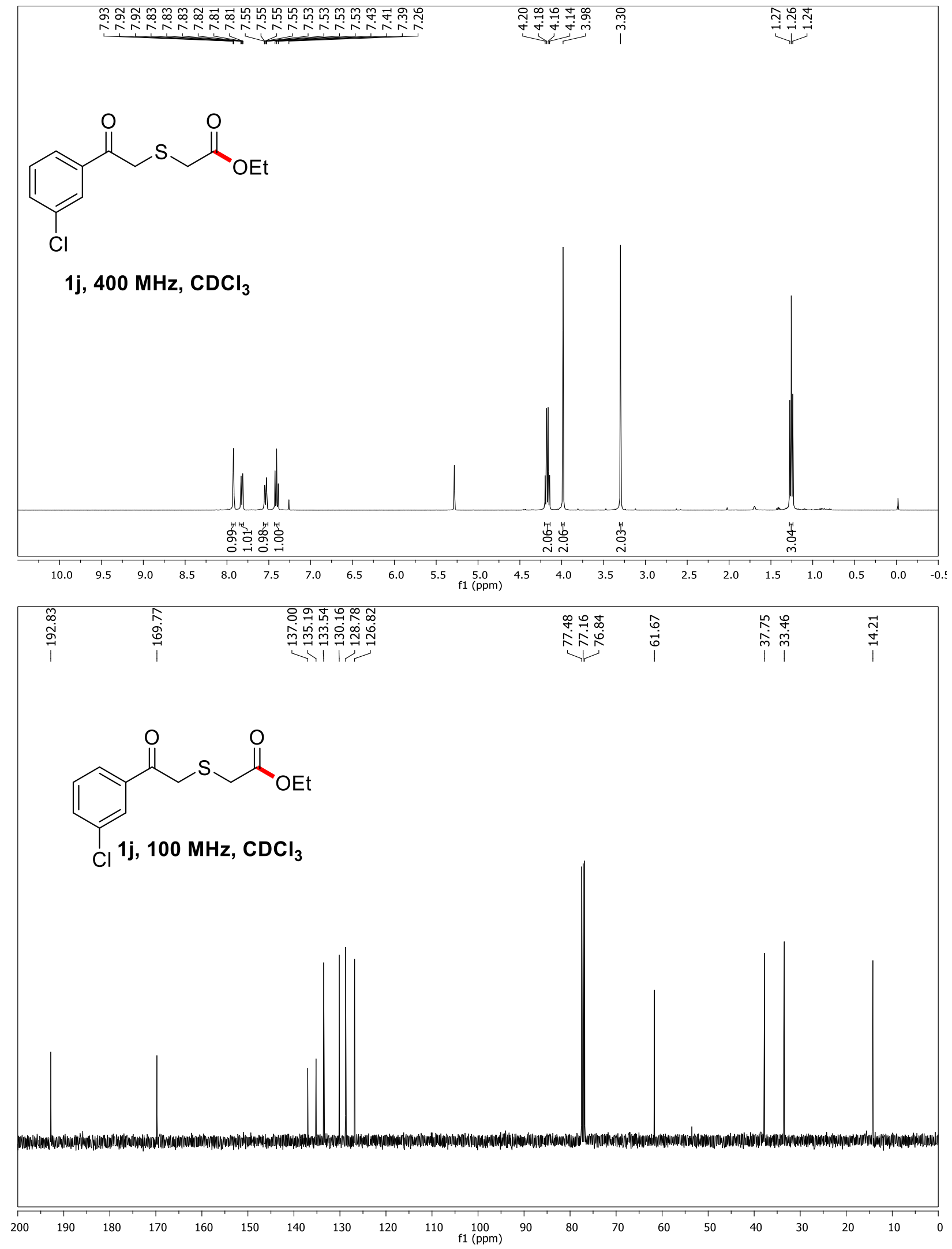
Ethyl 2-((2-(3-nitrophenyl)-2-oxoethyl)thio)acetate (1k)
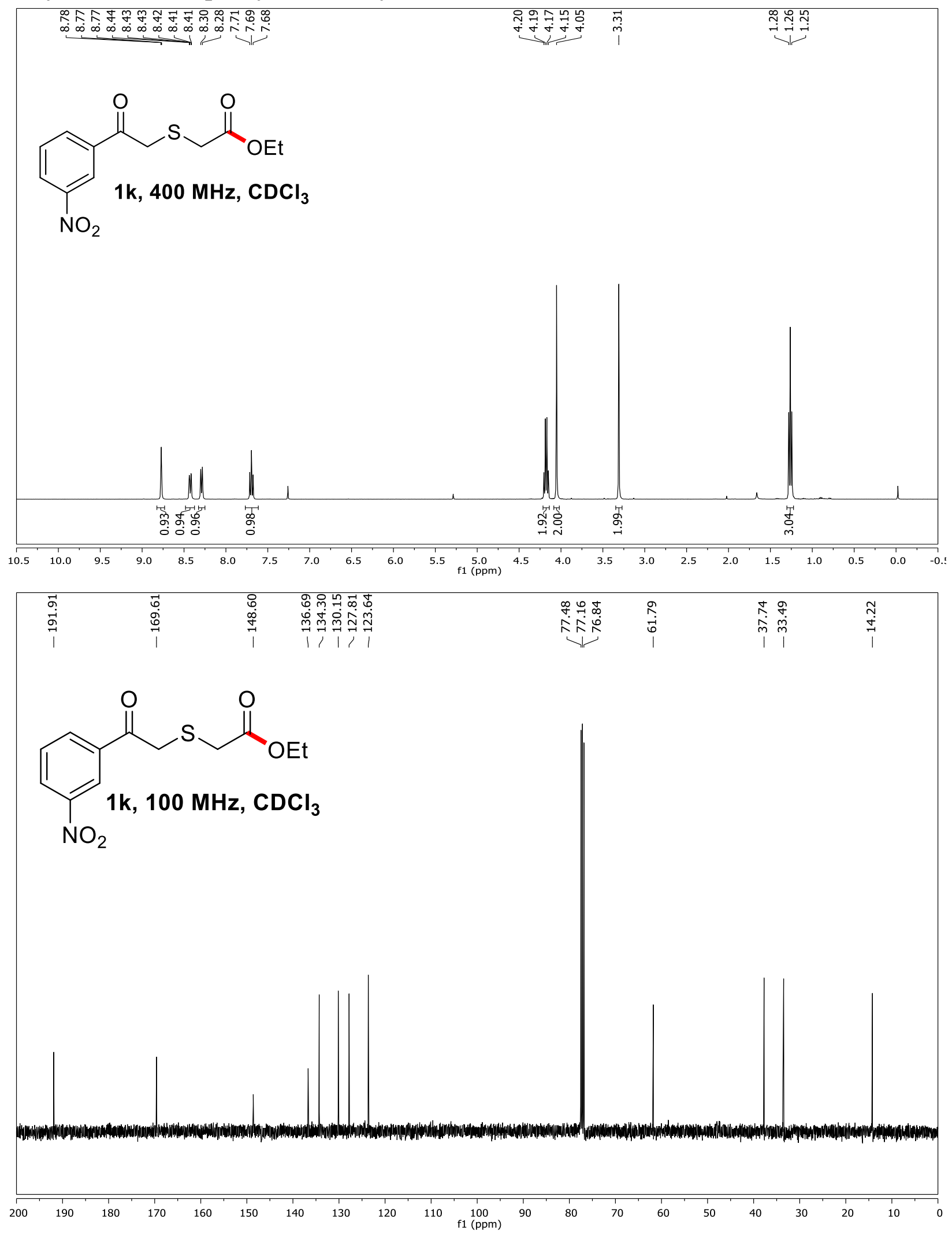
Ethyl 2-((2-(2-methoxyphenyl)-2-oxoethyl)thio)acetate (11)
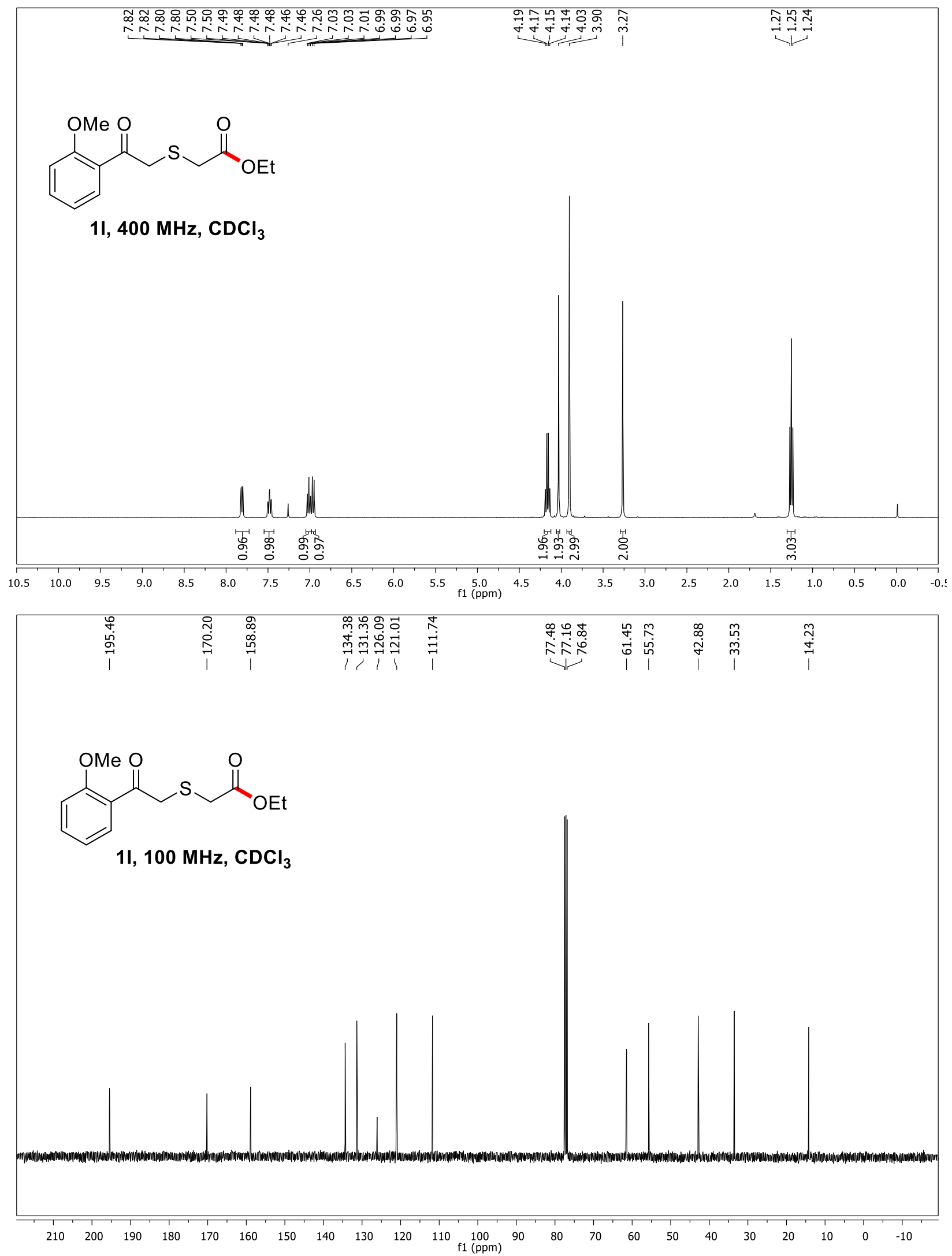
Ethyl 2-((2-(2-chlorophenyl)-2-oxoethyl)thio)acetate (1m)
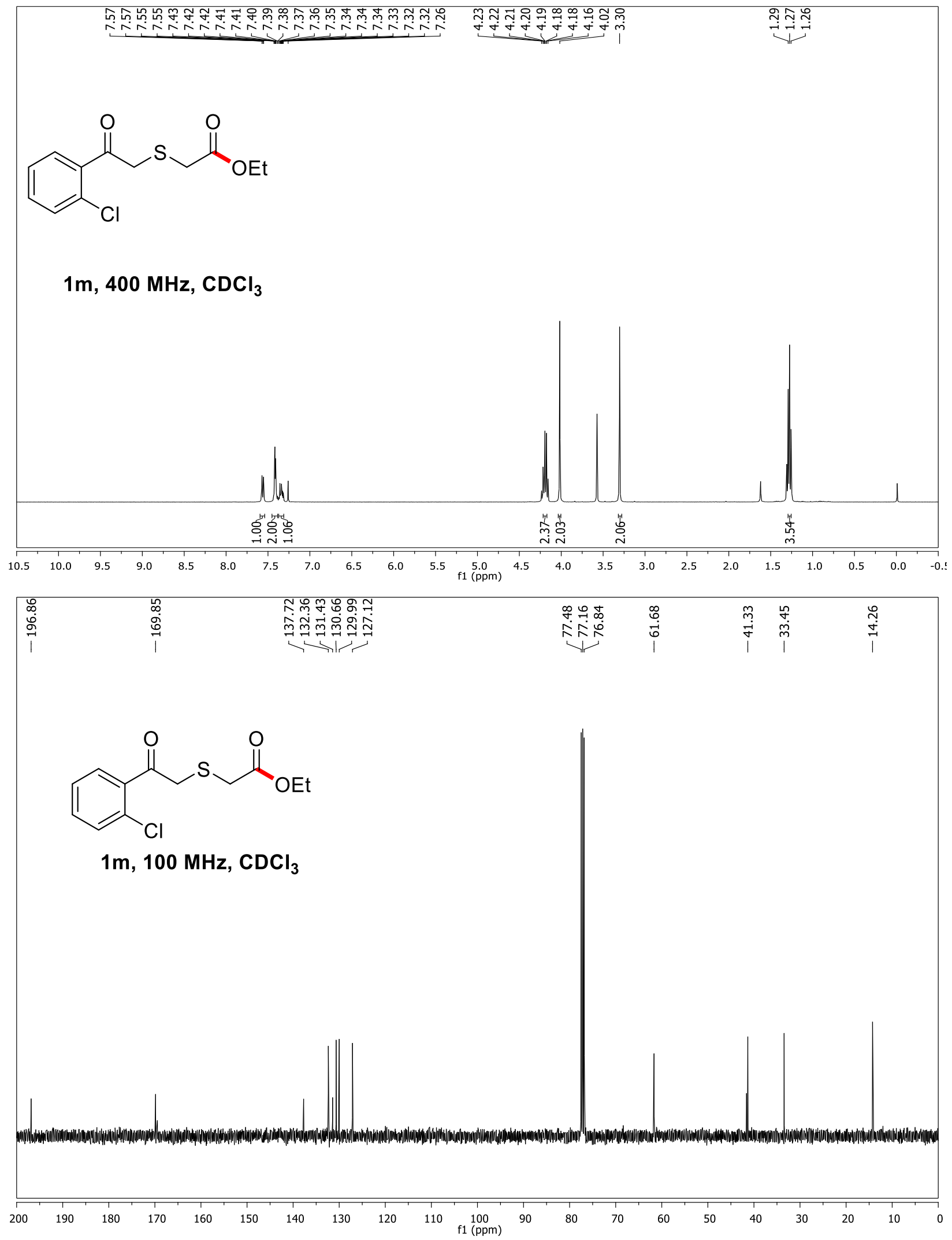
Ethyl 2-((2-(2-nitrophenyl)-2-oxoethyl)thio)acetate (1n)
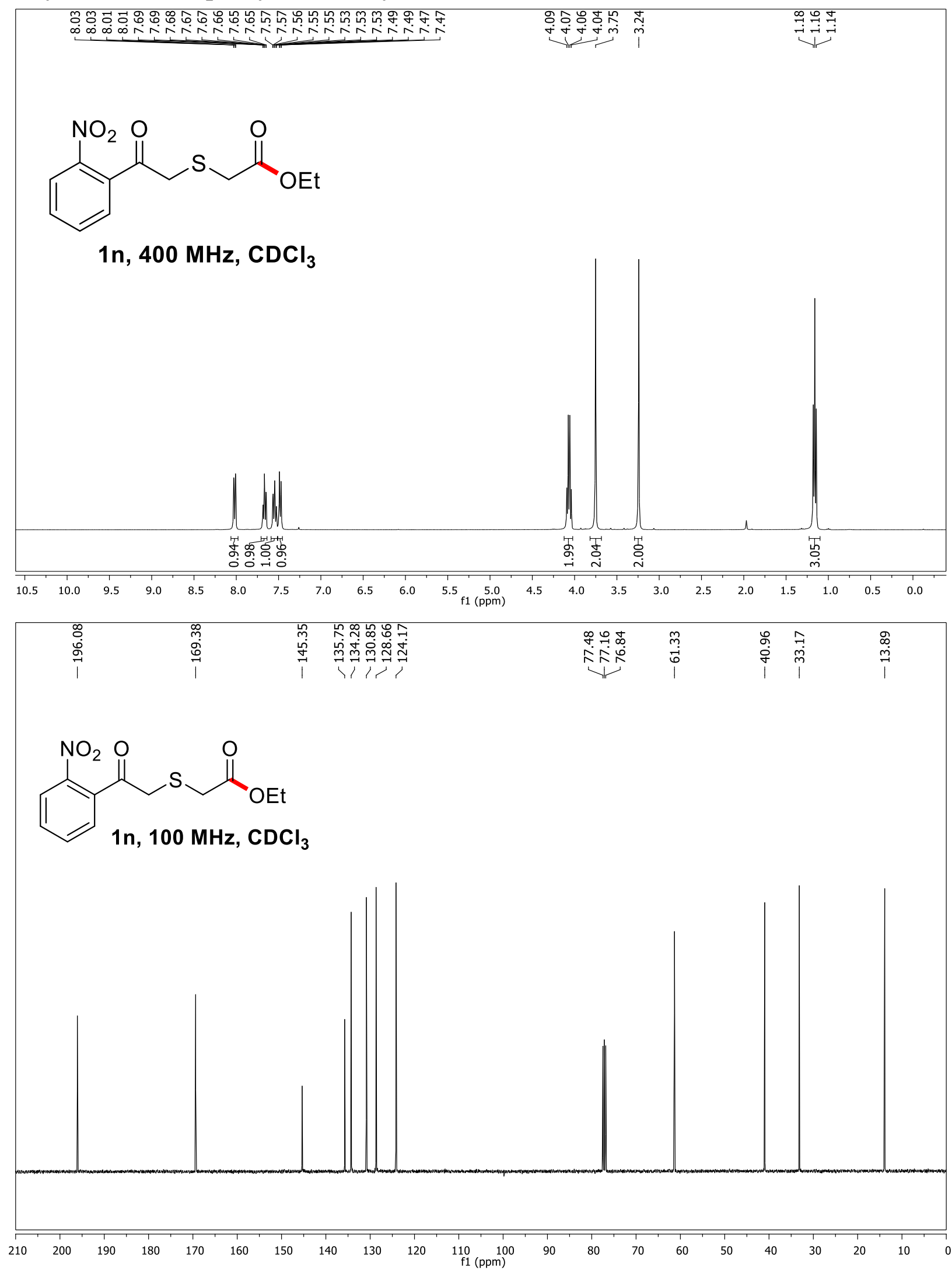
Ethyl 2-((2-(3,4-dimethoxyphenyl)-2-oxoethyl)thio)acetate (10)
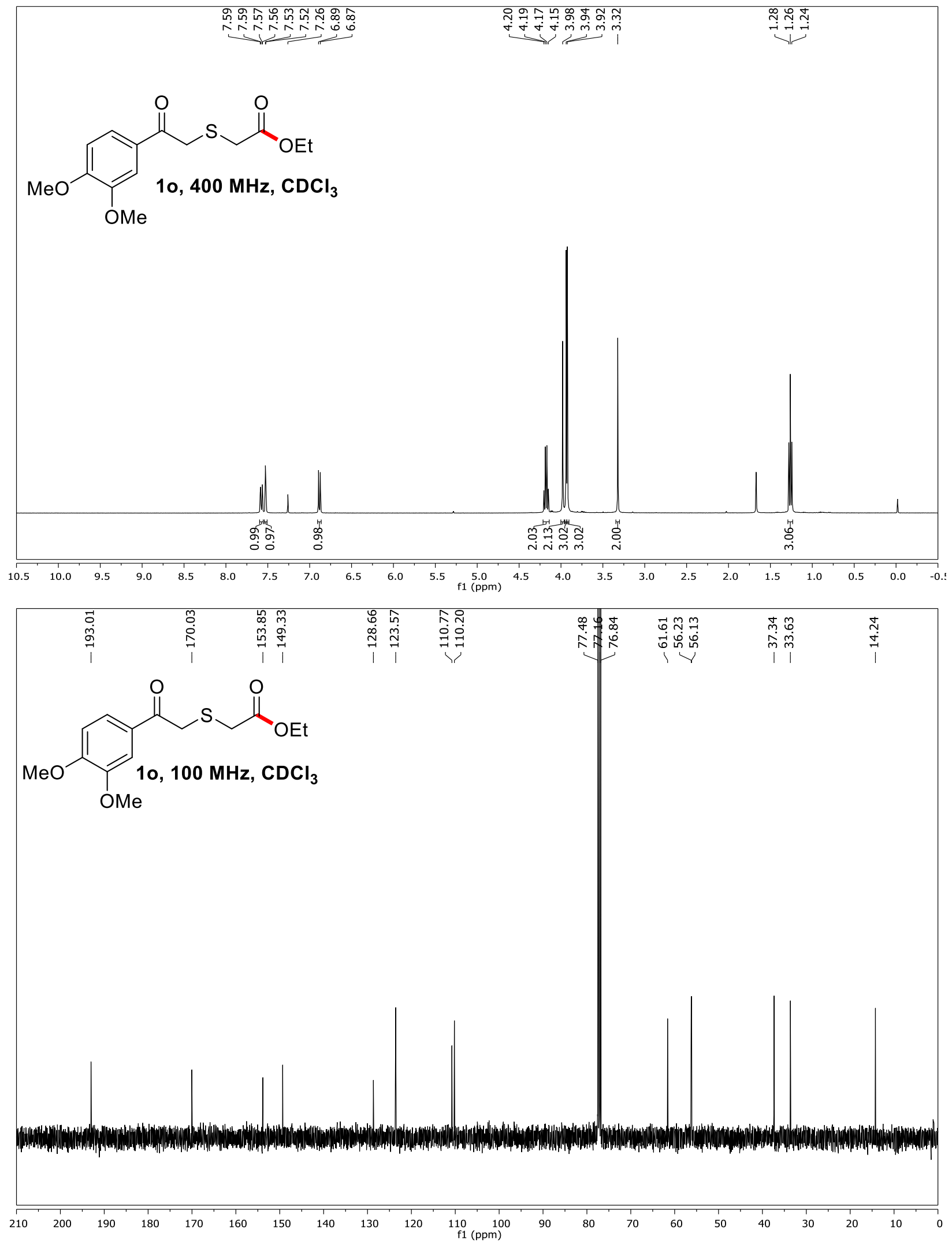
Ethyl 2-((2-(3-bromo-4-fluorophenyl)-2-oxoethyl)thio)acetate (1p)
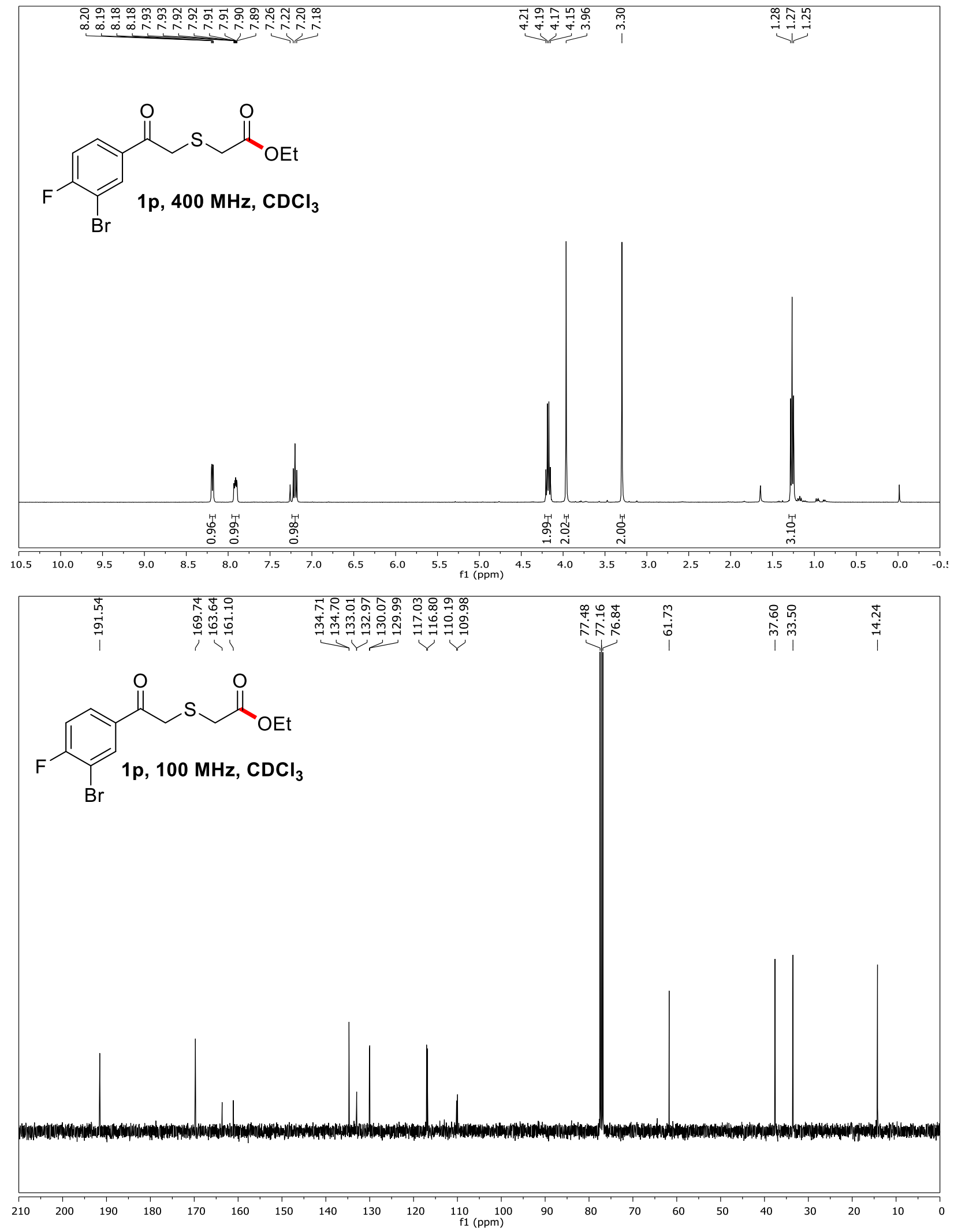
Ethyl 2-((2-(naphthalen-2-yl)-2-oxoethyl)thio)acetate(1q)
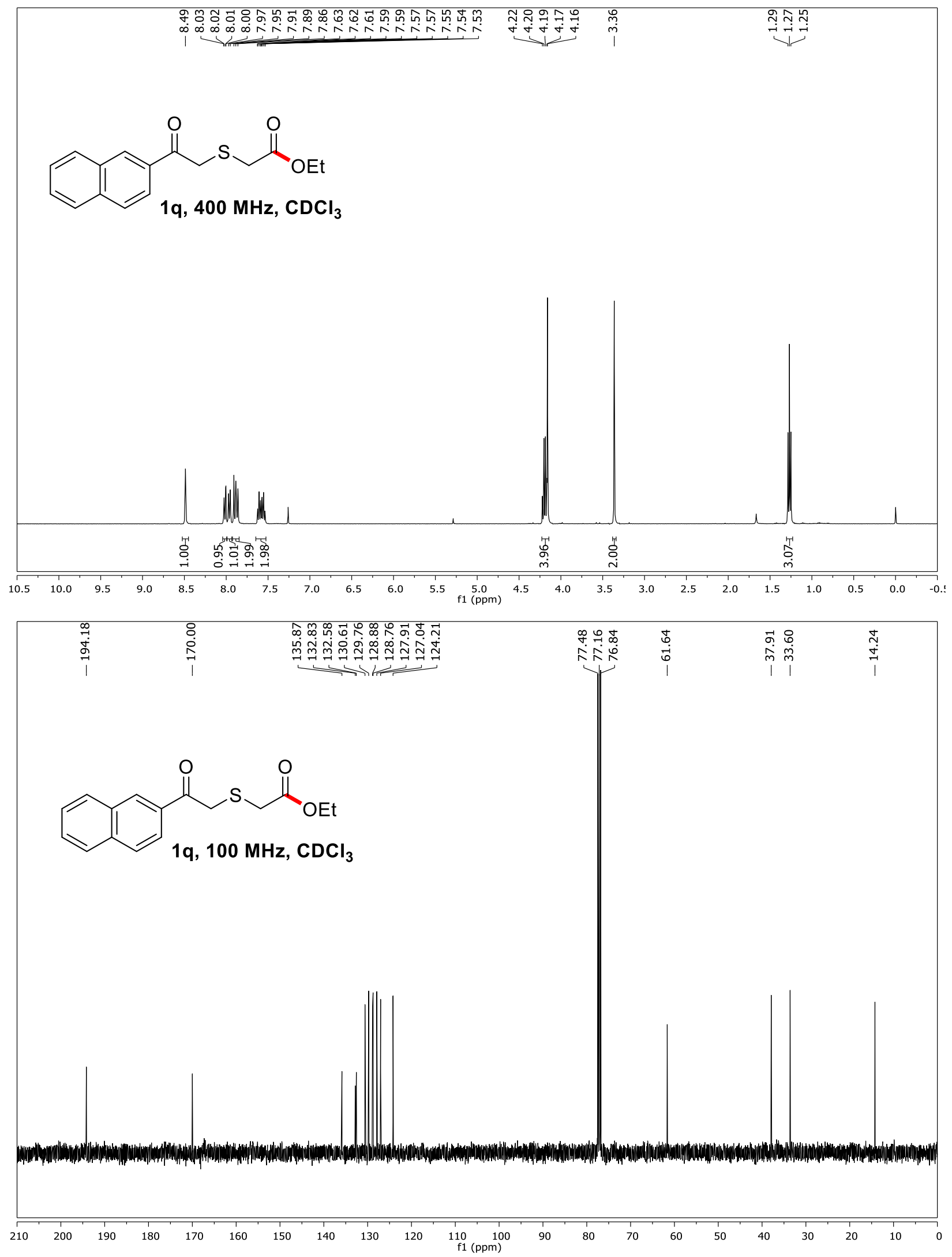
Ethyl 2-((2-(naphthalen-1-yl)-2-oxoethyl)thio)acetate (1r)
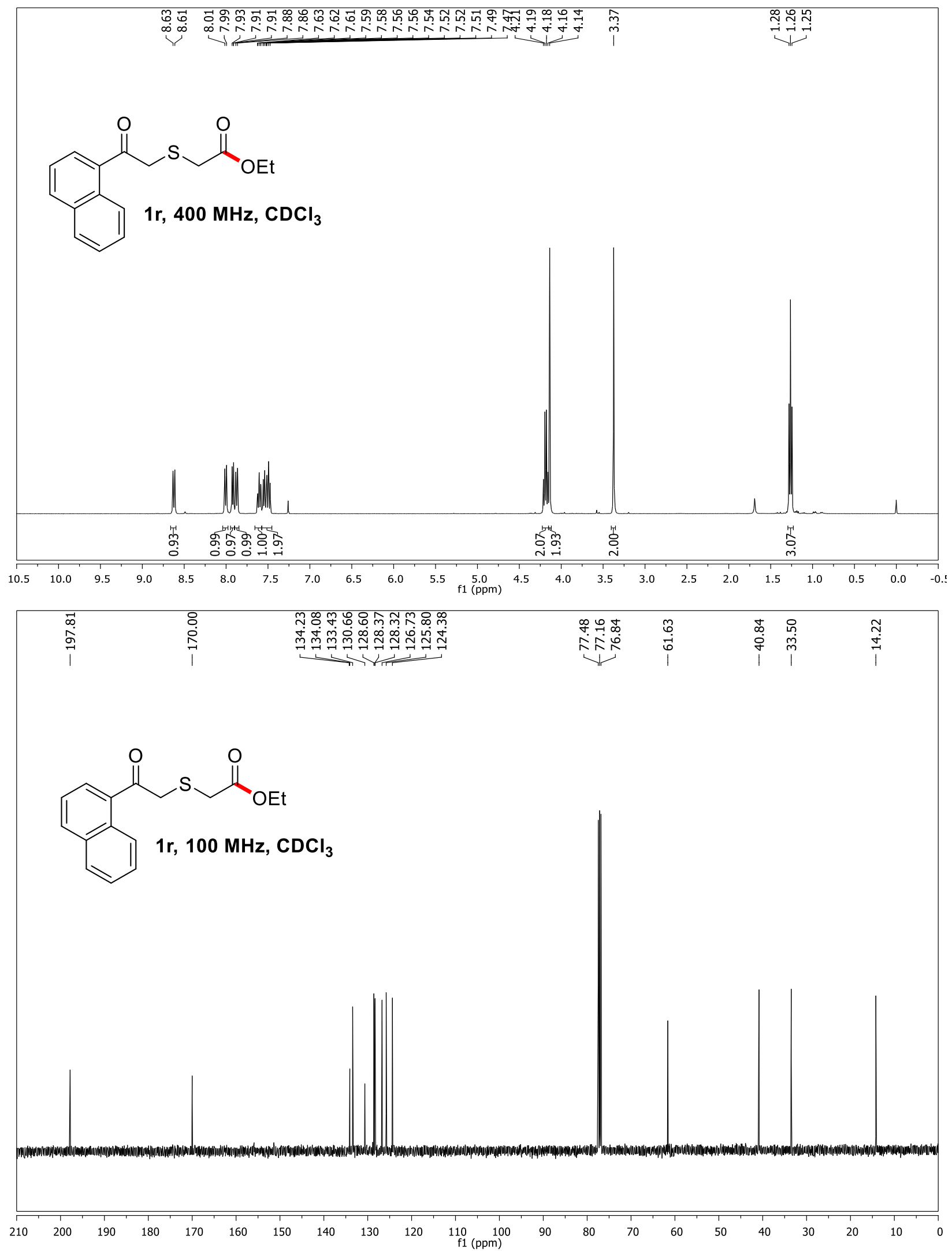
Ethyl 2-((2-(3,5-difluorophenyl)-2-oxoethyl)thio)acetate (1s)
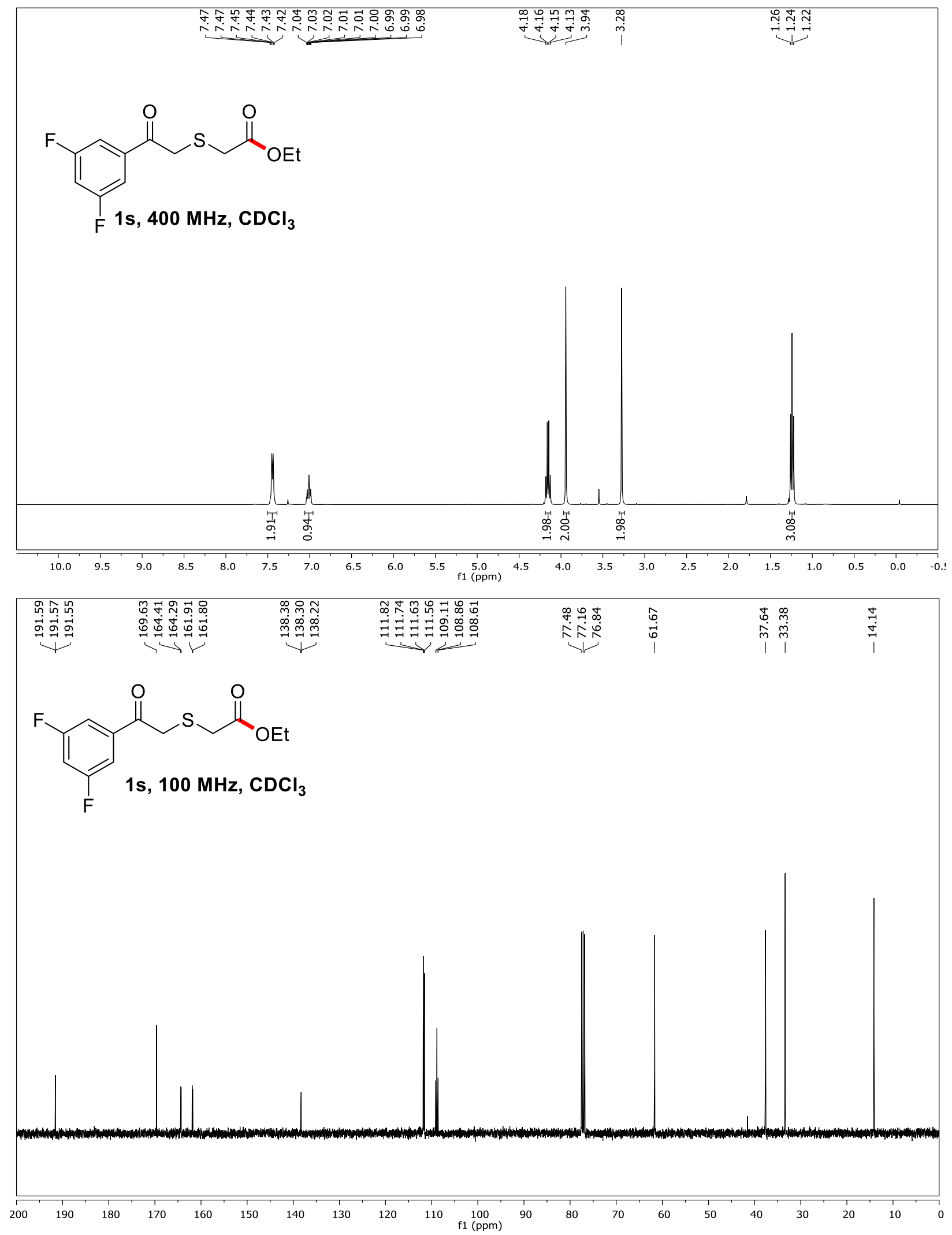
Ethyl 2-((2-oxo-2-(thiophen-2-yl)ethyl)thio)acetate (1t)
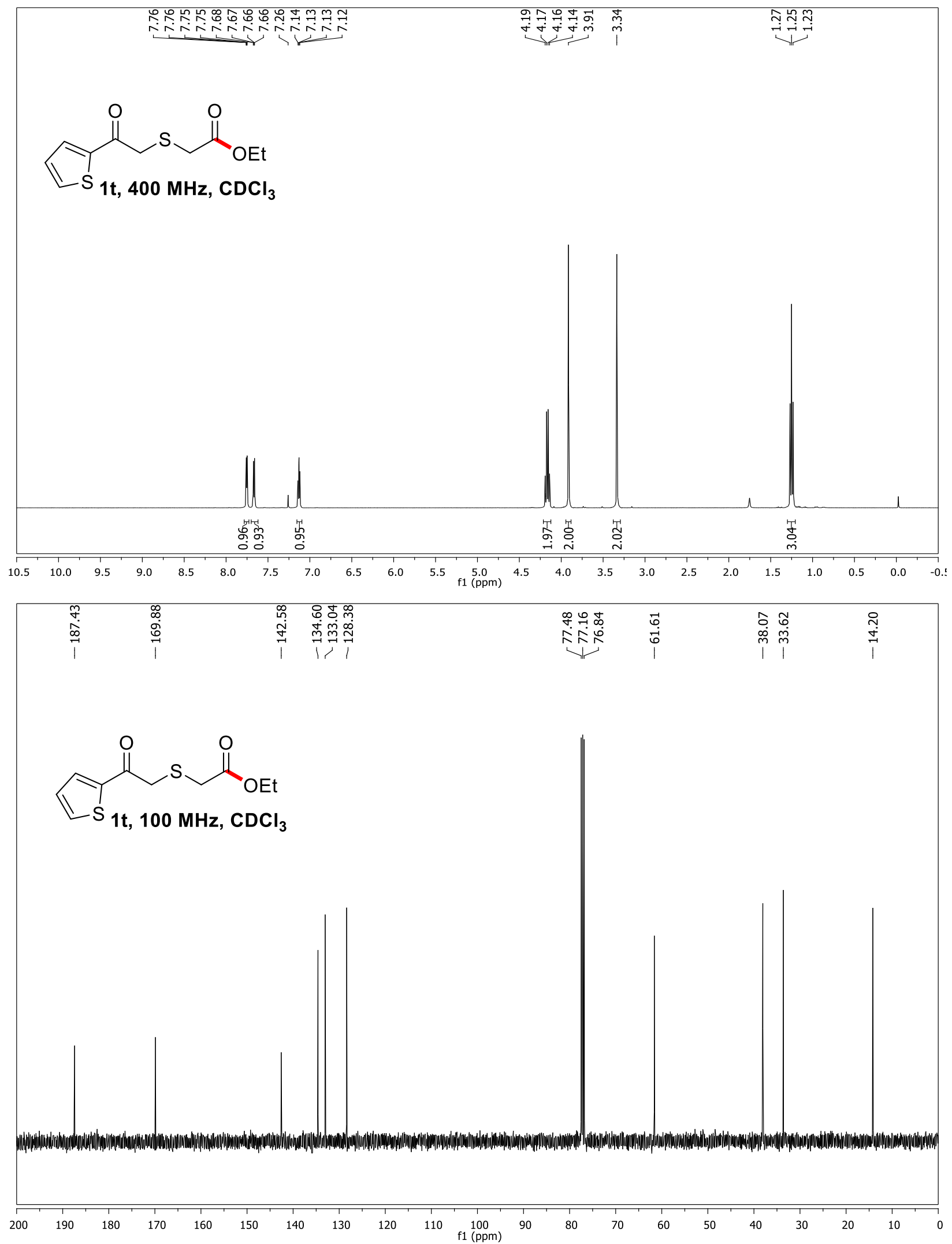
Methyl 2-((2-oxo-2-phenylethyl)thio)acetate (1u)
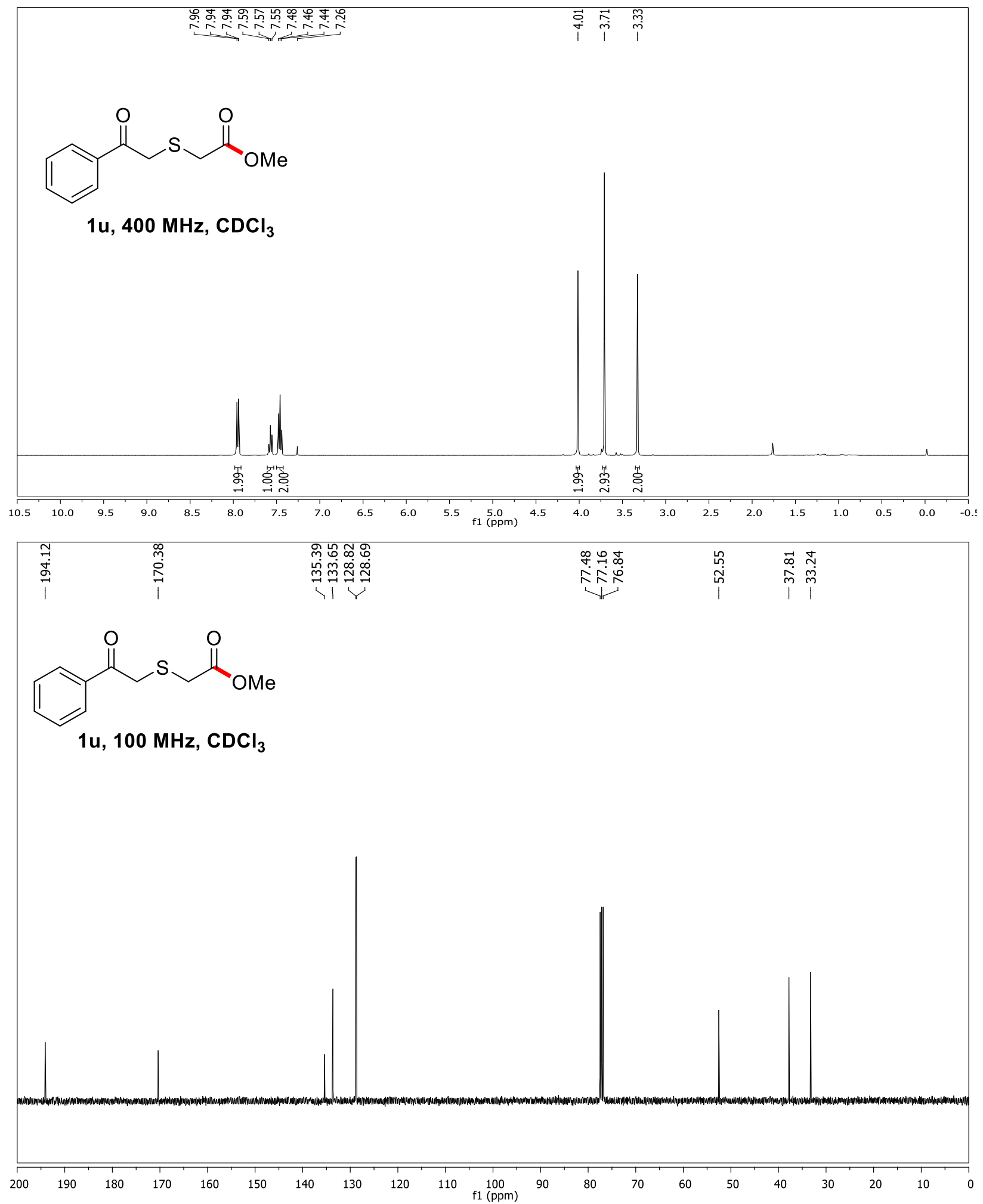
Butyl 2-((2-oxo-2-phenylethyl)thio)acetate (1v)

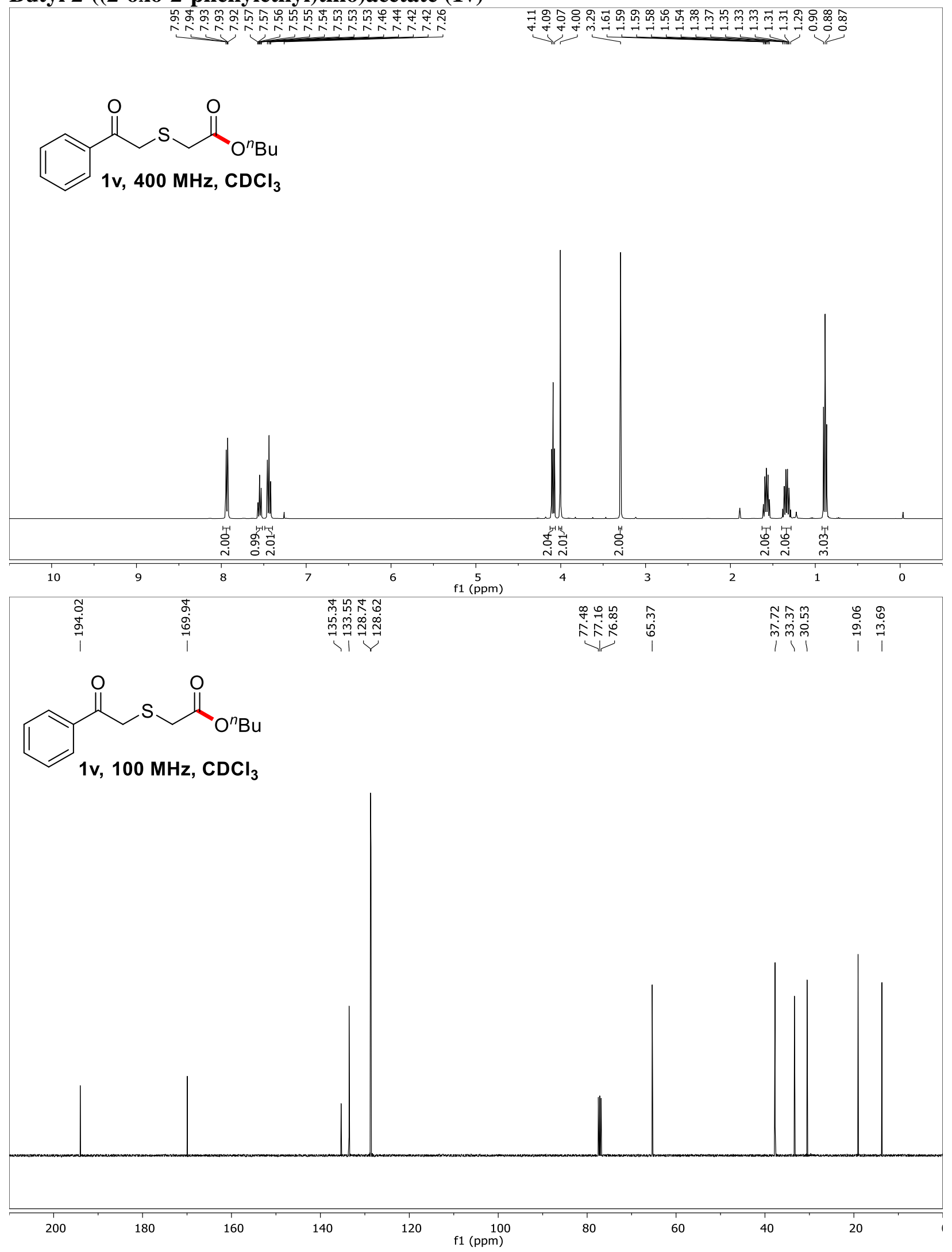


Isopropyl 2-((2-oxo-2-phenylethyl)thio)acetate (1w)
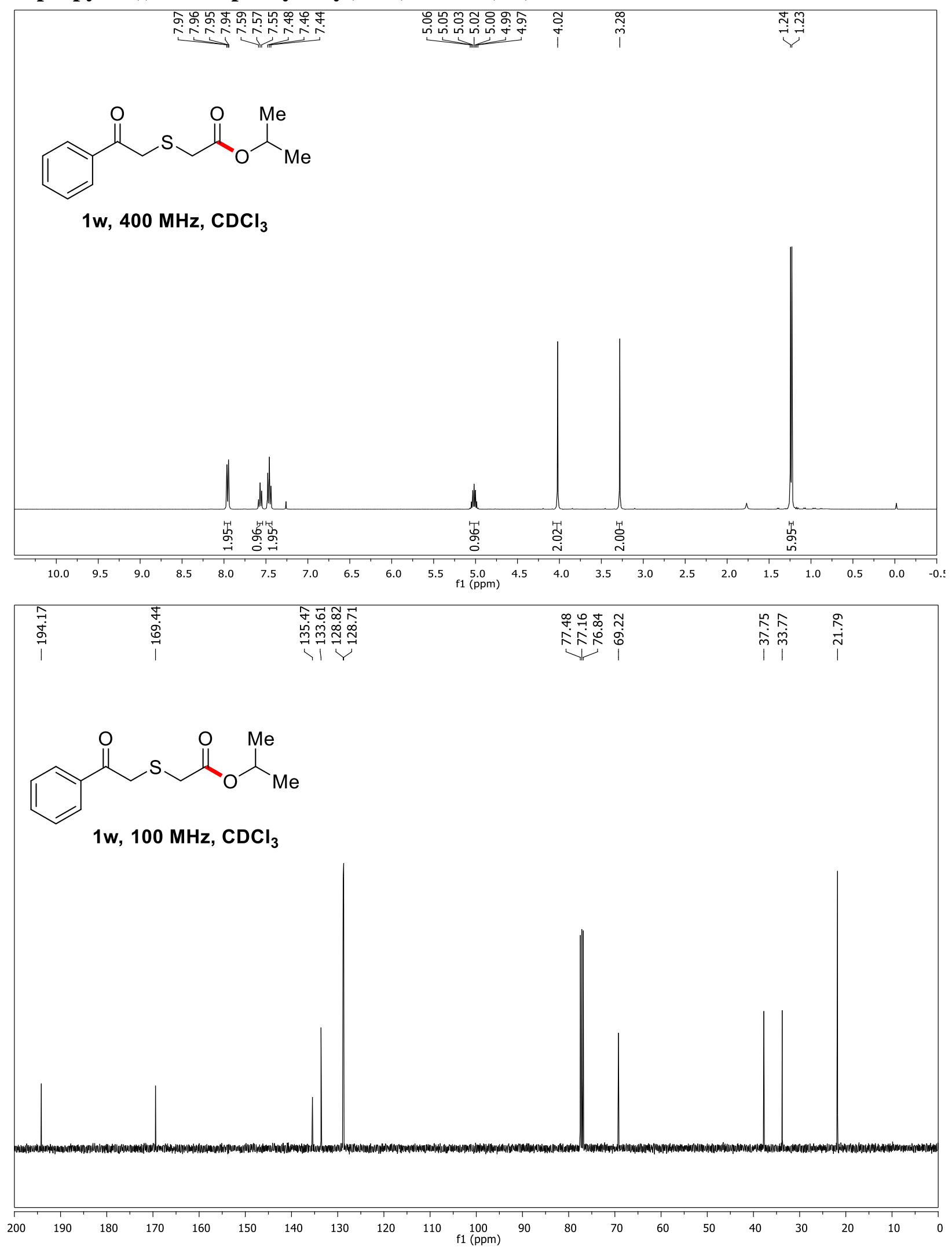
Allyl 2-((2-oxo-2-phenylethyl)thio)acetate (1x)
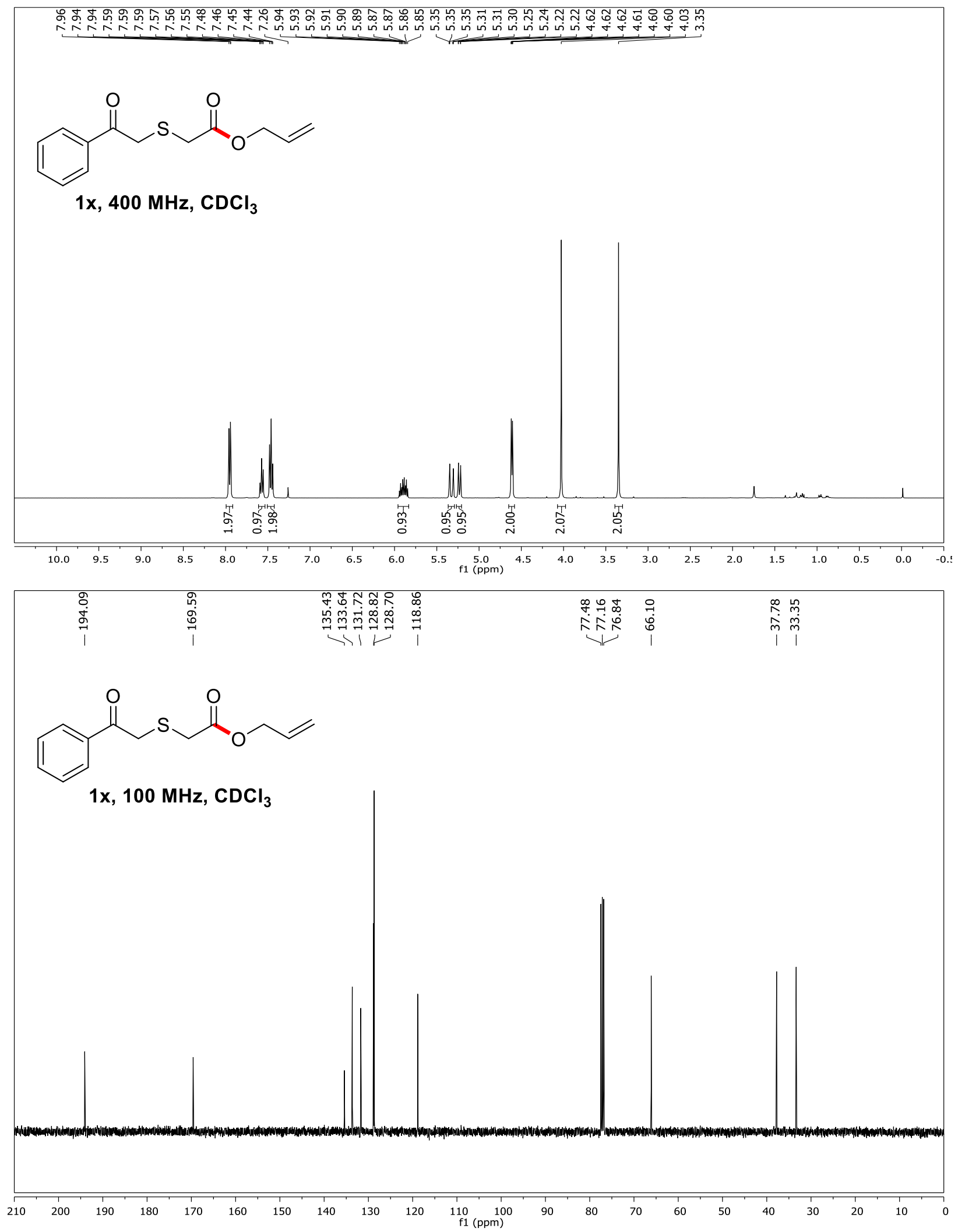
Prop-2-yn-1-yl 2-((2-oxo-2-phenylethyl)thio)acetate (1y)
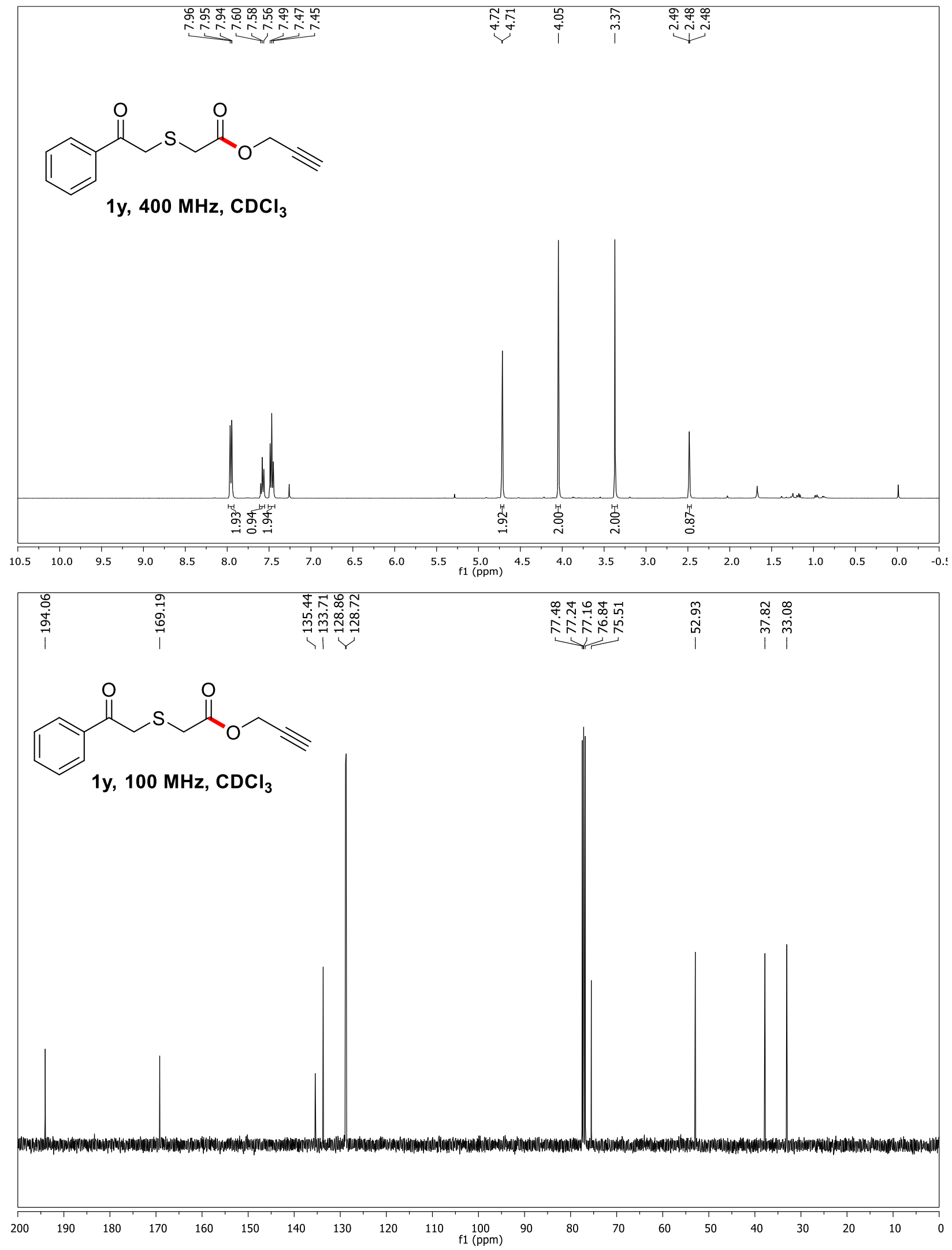
Phenethyl 2-((2-oxo-2-phenylethyl)thio)acetate (1z)
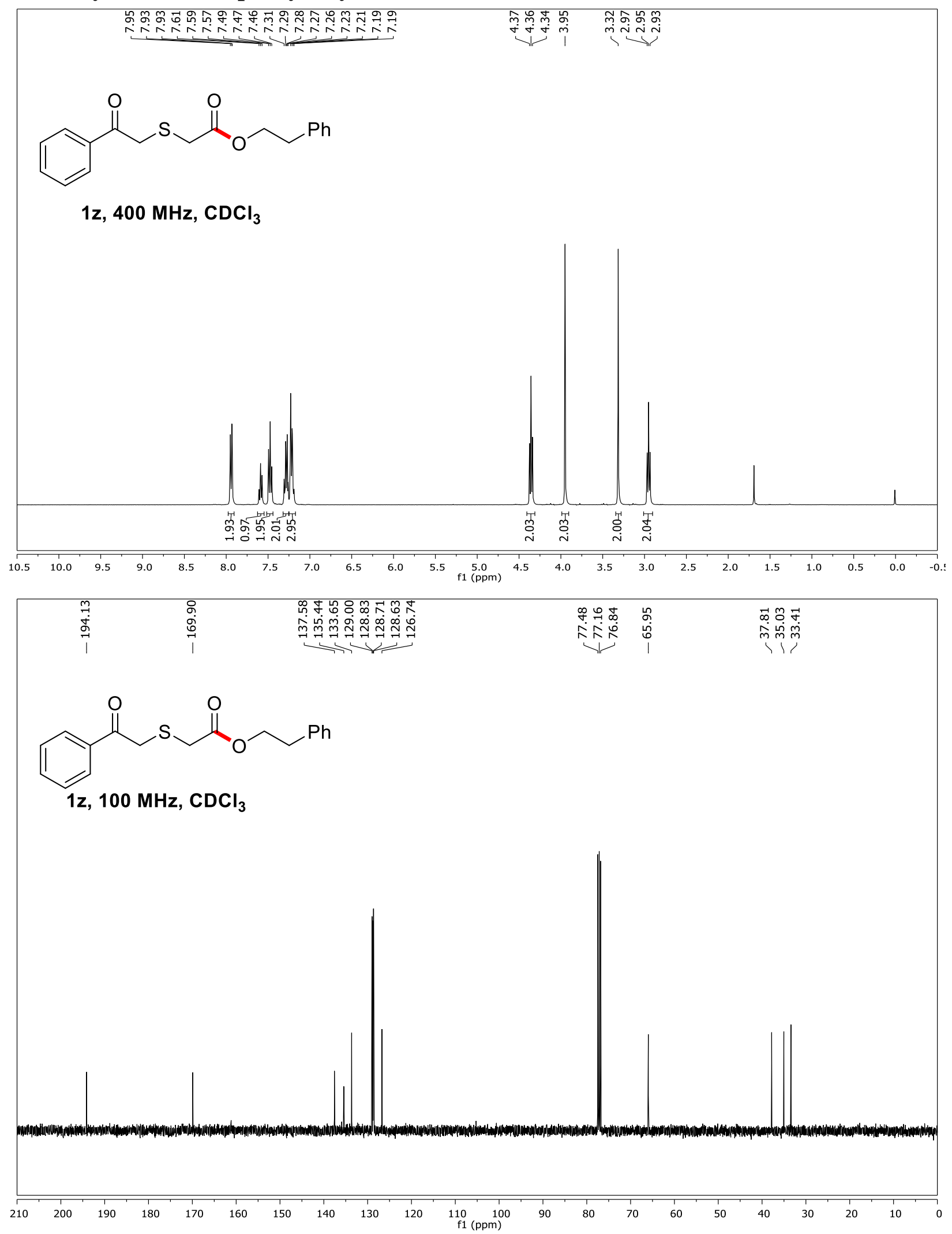
Phenyl 2-((2-oxo-2-phenylethyl)thio)acetate (1aa)
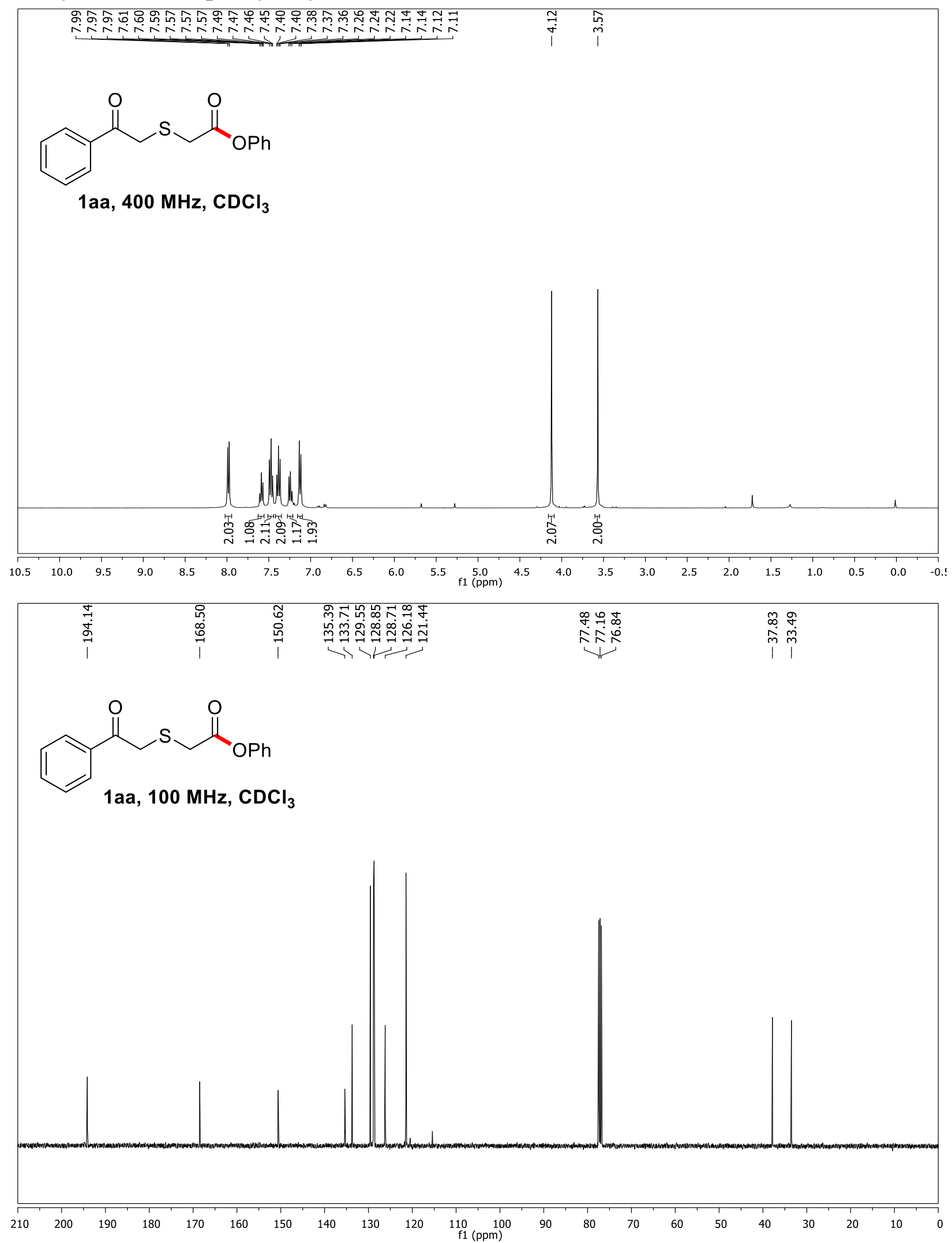

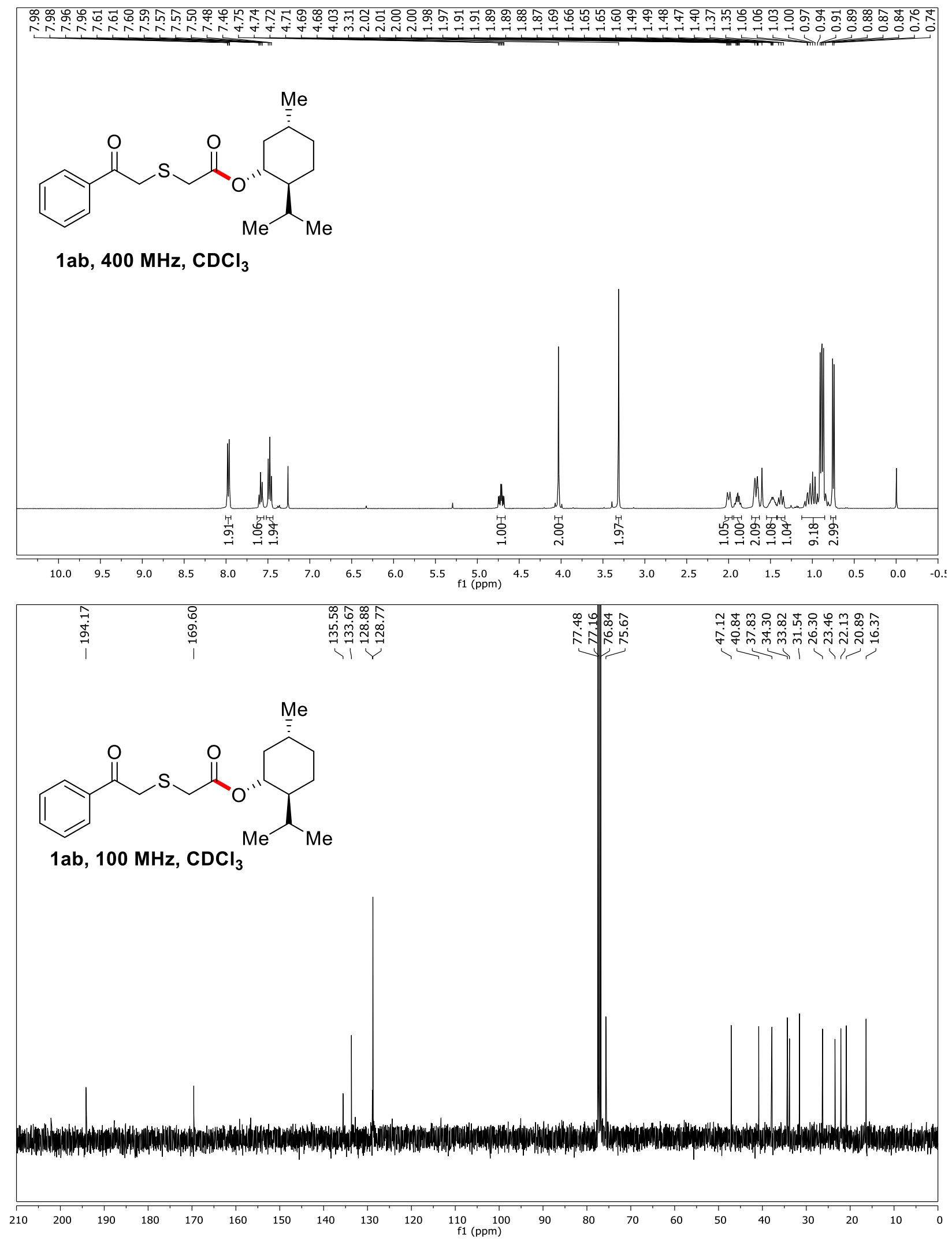
(E)-3,7-Dimethylocta-2,6-dien-1-yl 2-((2-oxo-2-phenylethyl)thio)acetate (1ac)
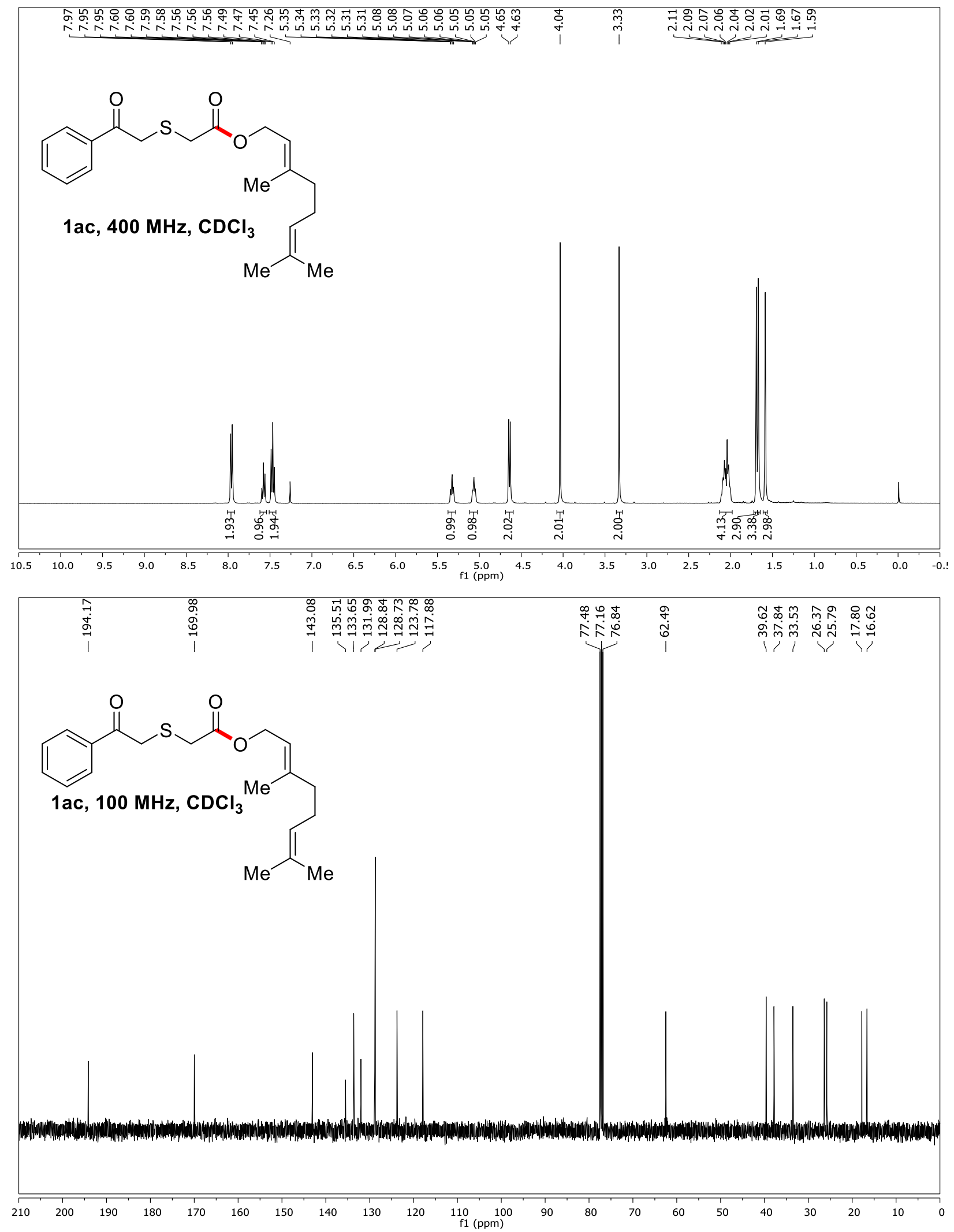
$(3 R, 8 S, 9 S, 10 R, 13 R, 14 S, 17 R)-10,13-D i m e t h y l-17-((R)$-6-methylheptan-2-

yl) 2,3,4,7,8,9,10,11,12,13,14,15,16,17-tetradecahydro- $1 H$-cyclopenta[a]phenanthren-3-yl 2 ((2-oxo-2-phenylethyl)thio)acetate (1ad)
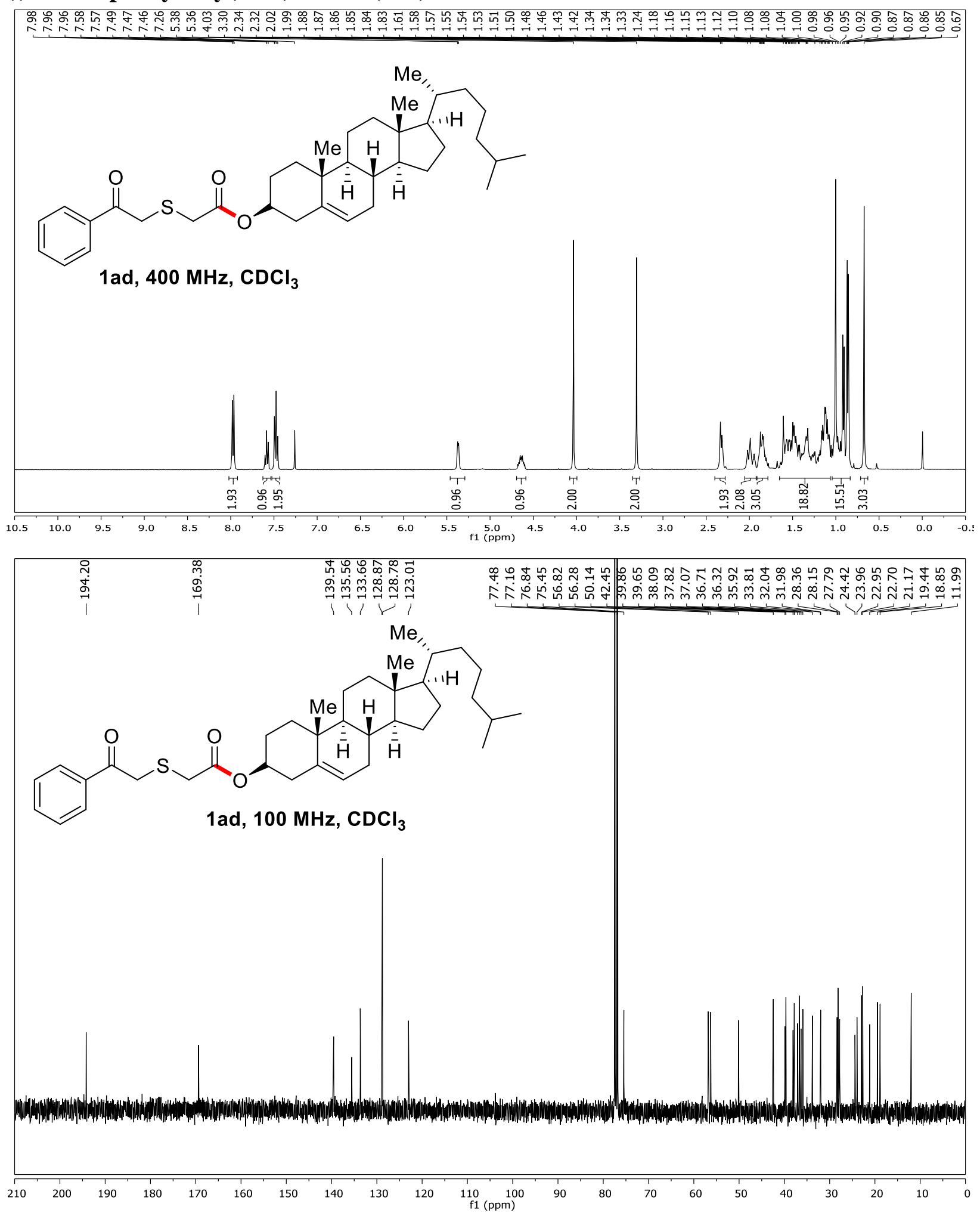
(R)-2-((Tert-butoxycarbonyl)amino)-3-phenylpropyl 2-((2-oxo-2-phenylethyl)thio) acetate (1ae)
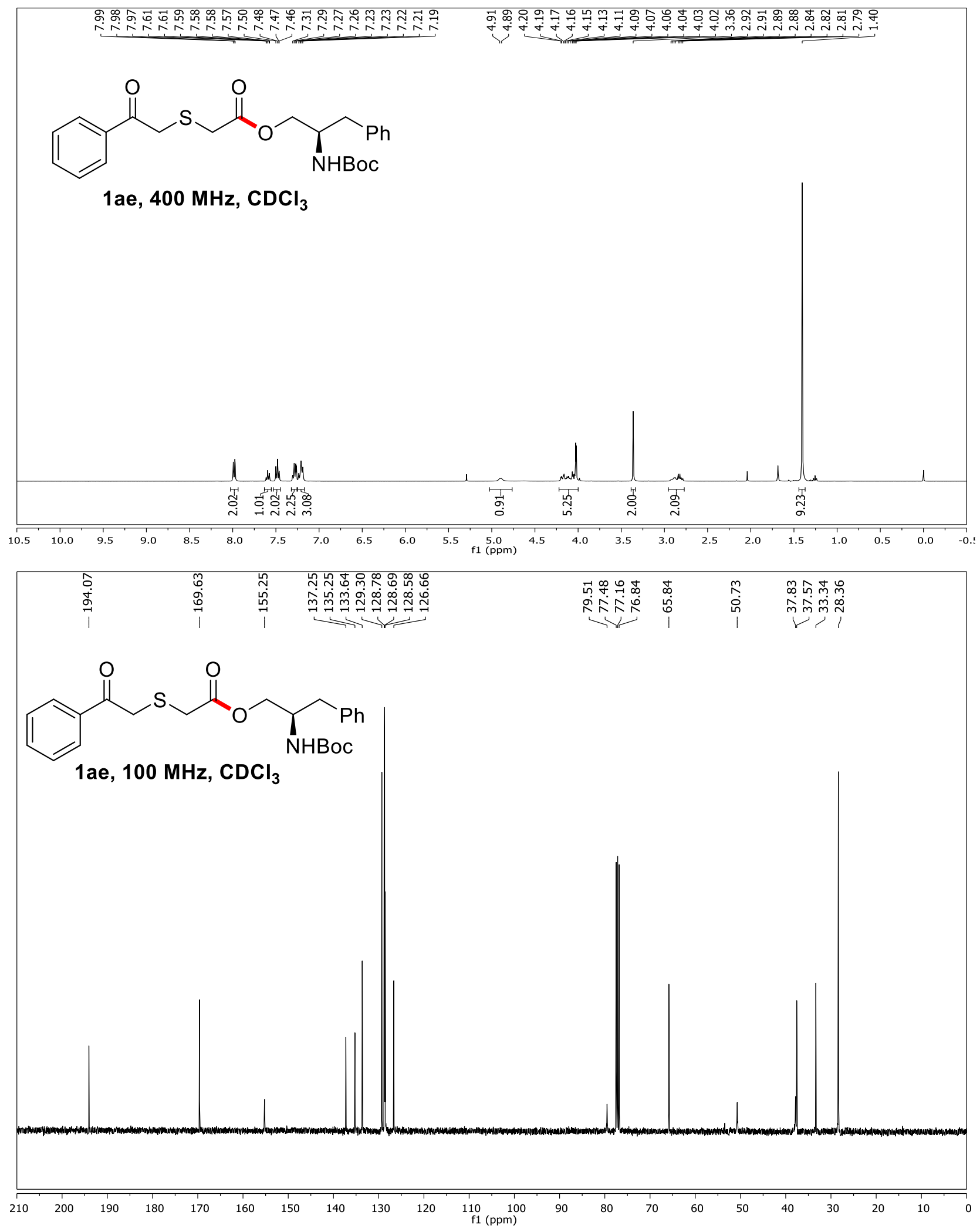
$\mathrm{N}, \mathrm{N}$-Dimethyl-2-((2-oxo-2-phenylethyl)thio)acetamide(1af)
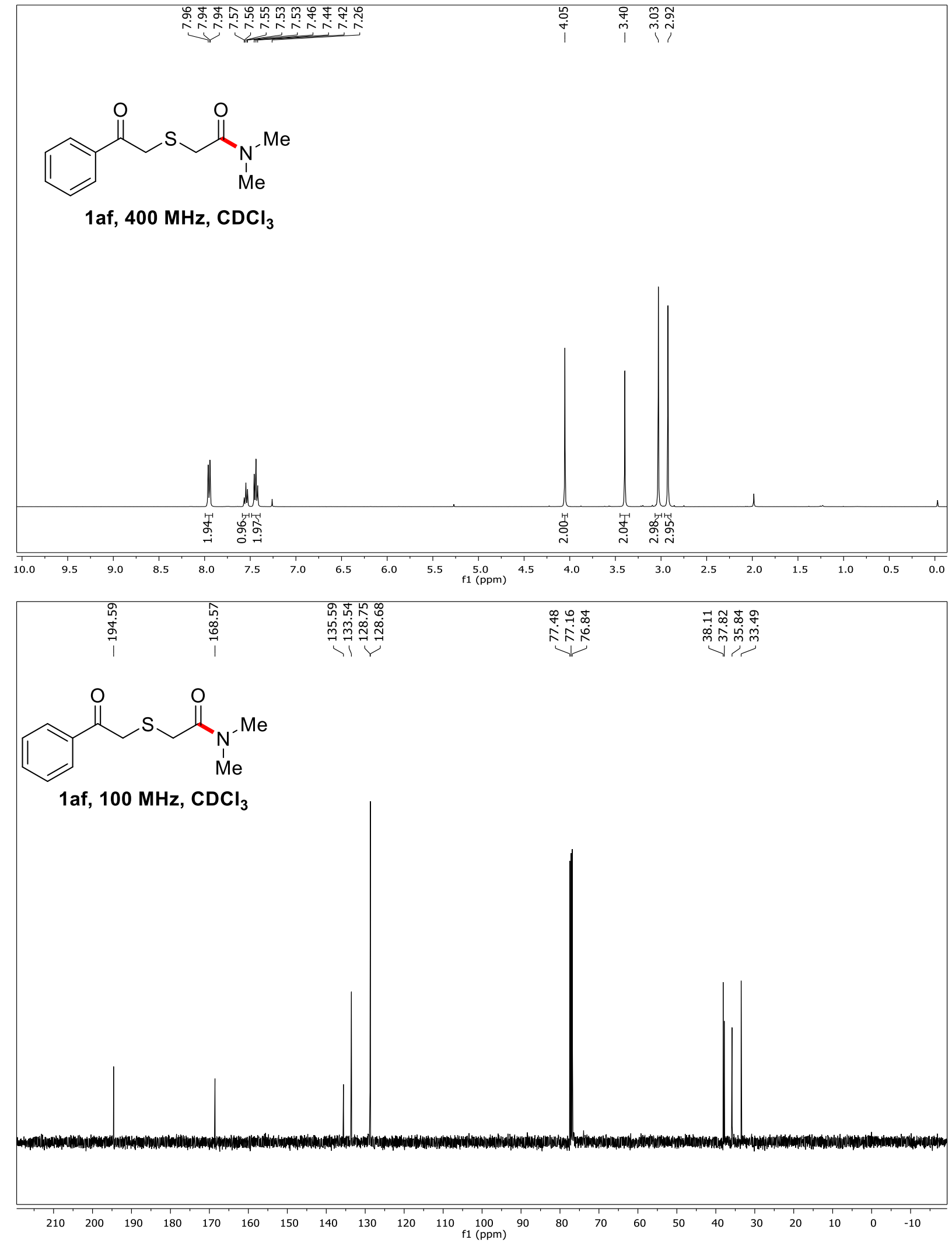
$N$-Methoxy- $N$-methyl-2-((2-oxo-2-phenylethyl)thio)acetamide (1ag)
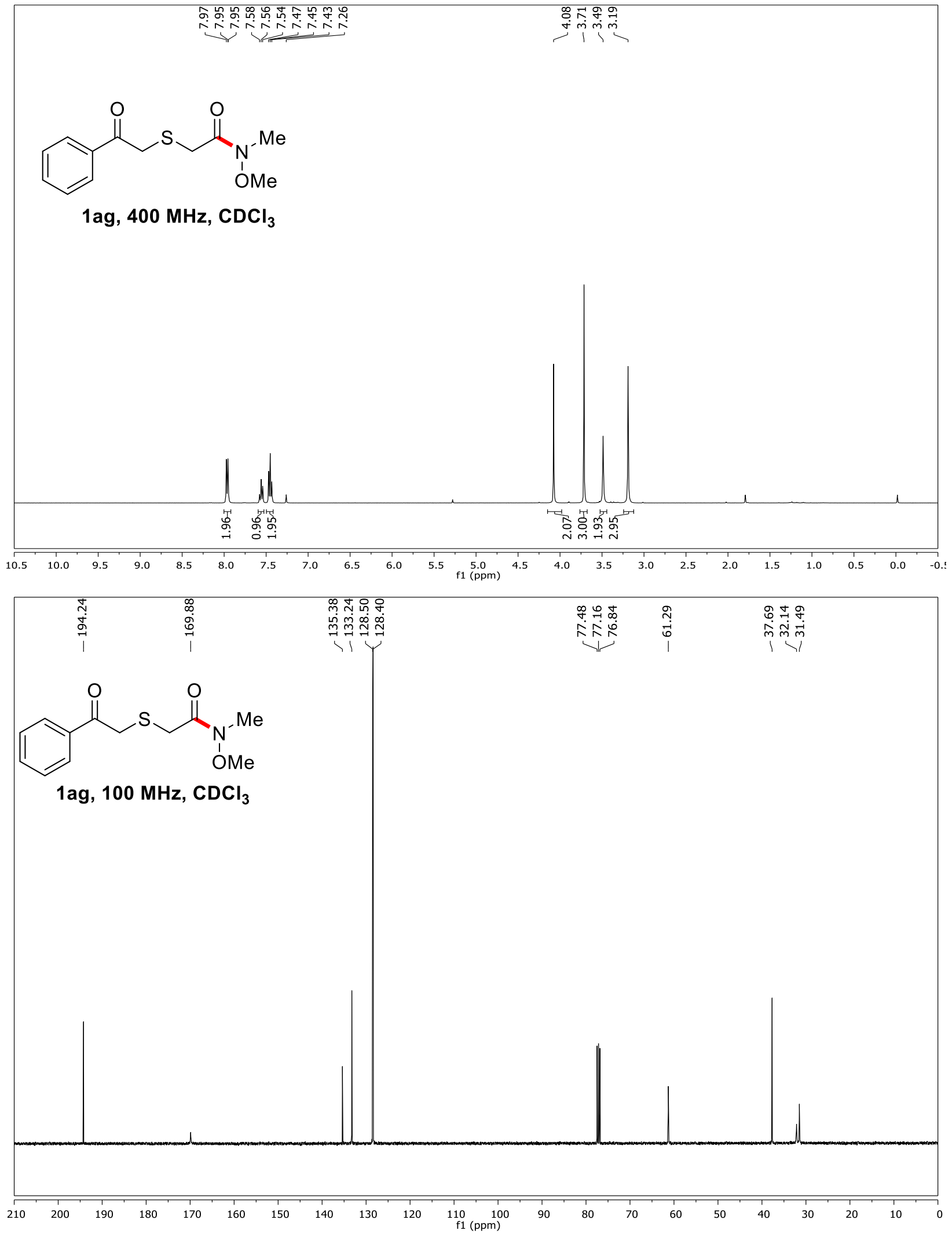
Ethyl 2-((2-oxocyclohexyl)thio)acetate (1ah)
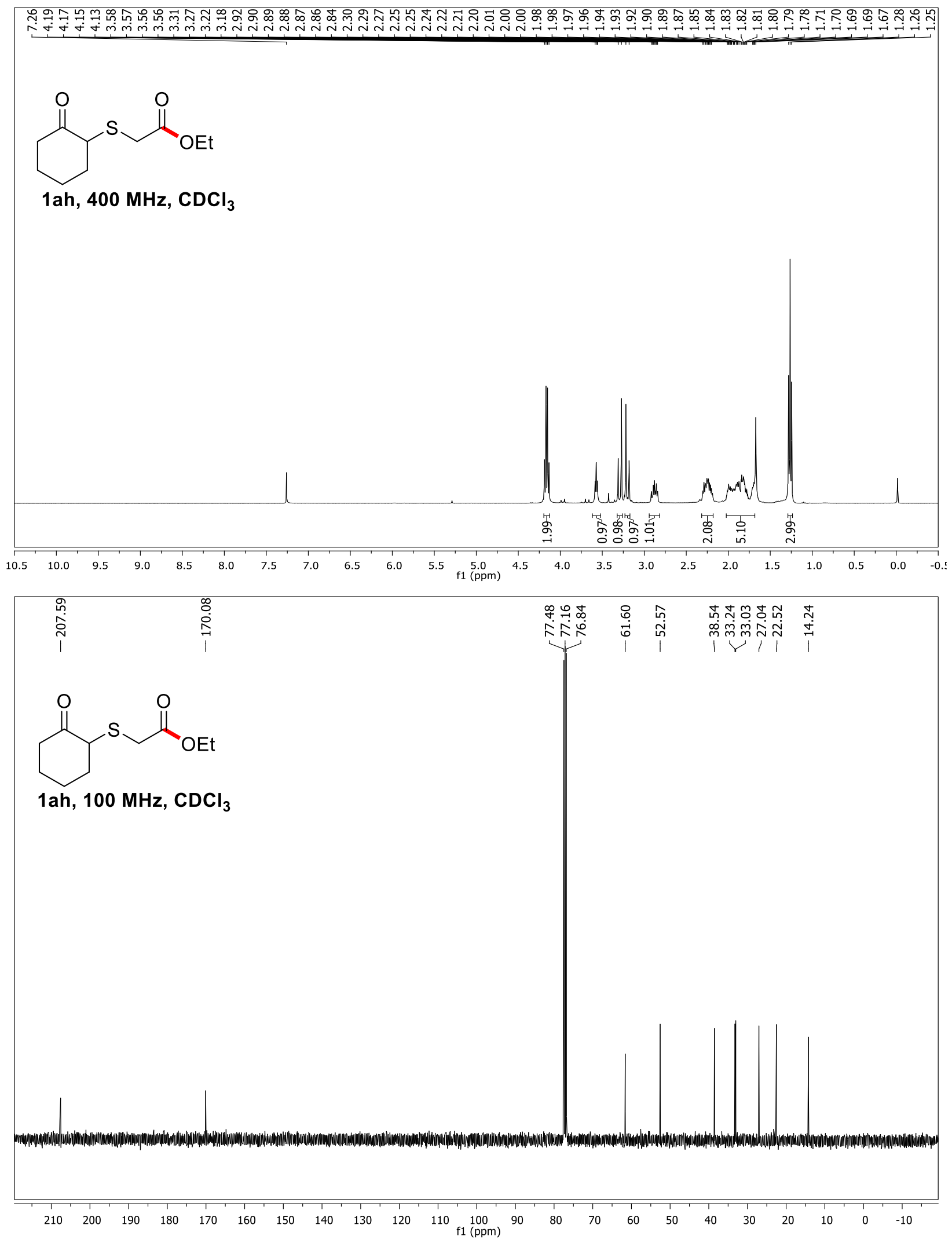


\section{Ethyl 2-((2-oxocycloheptyl)thio)acetate (1ai)}

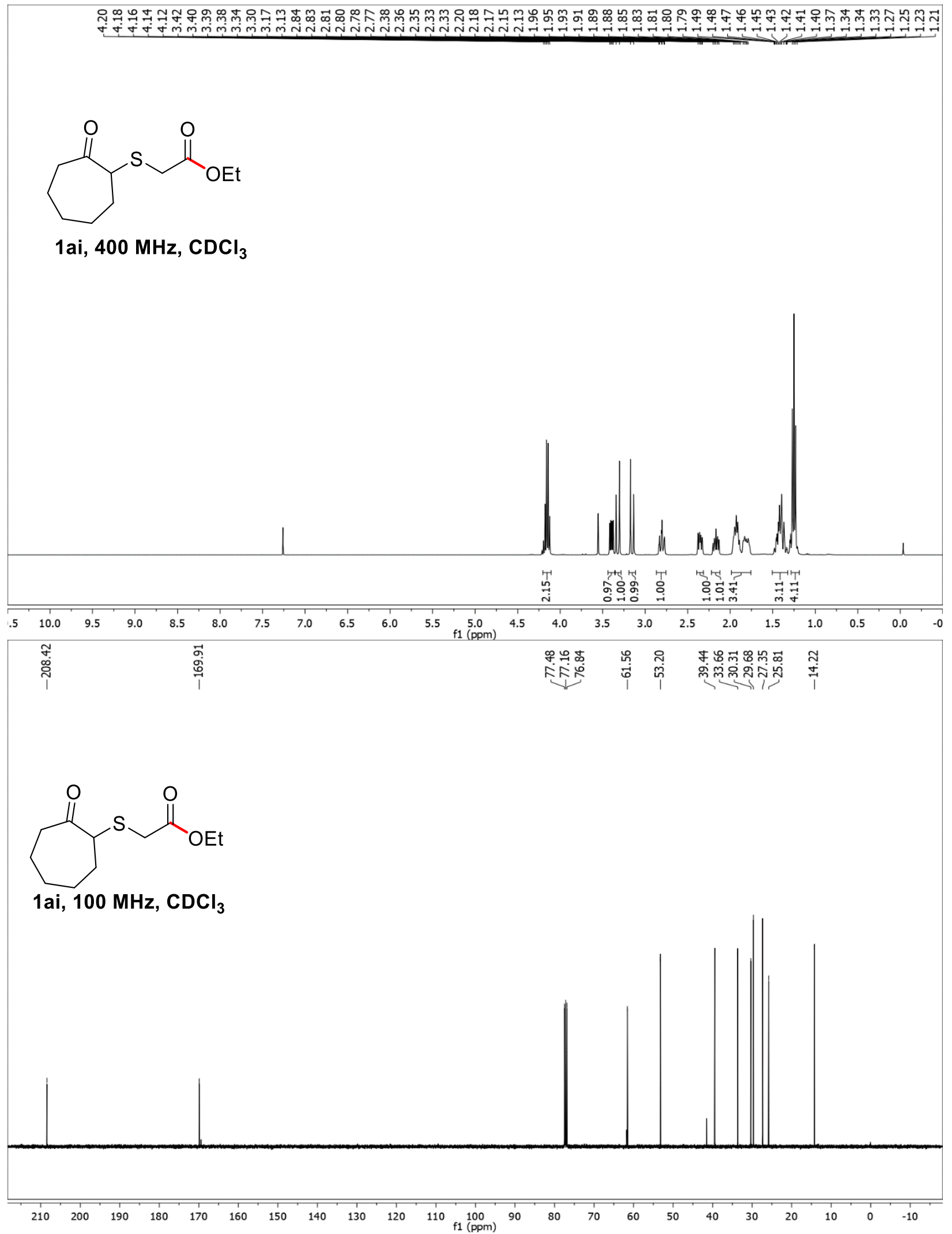


Ethyl 2-((1-oxo-2,3-dihydro-1H-inden-2-yl)thio)acetate (1aj)
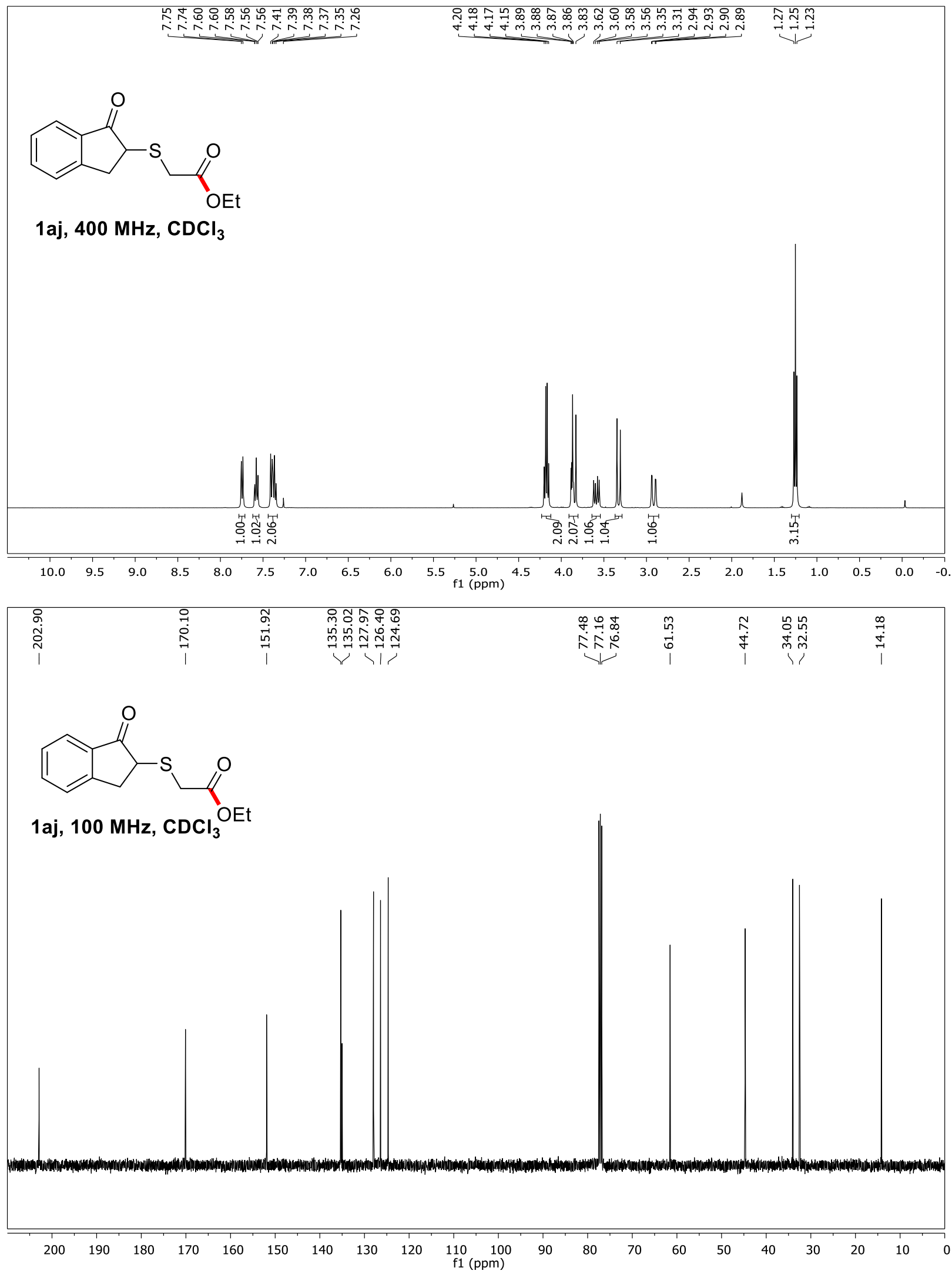

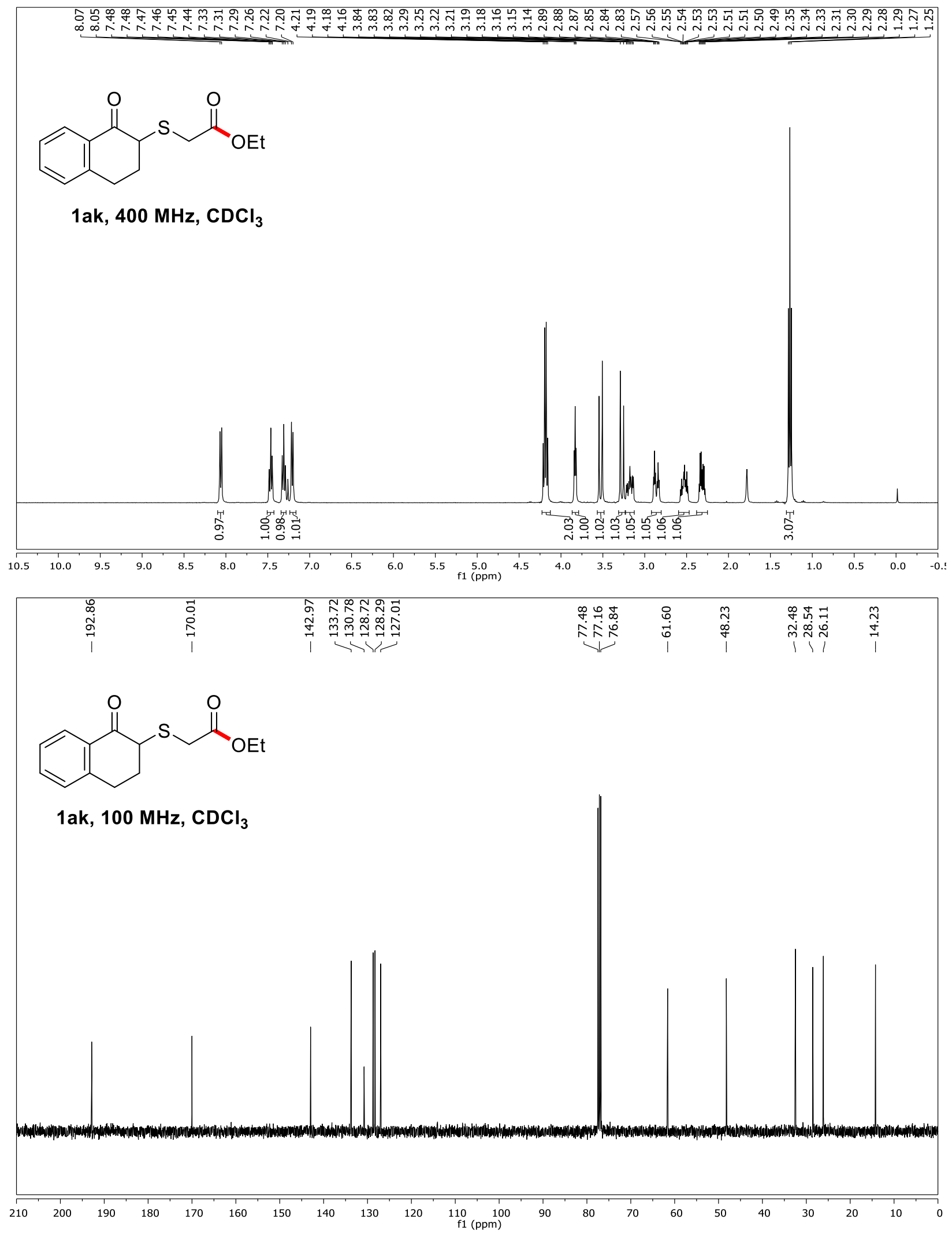
Ethyl 2-((2-oxopropyl)thio)acetate (1al)
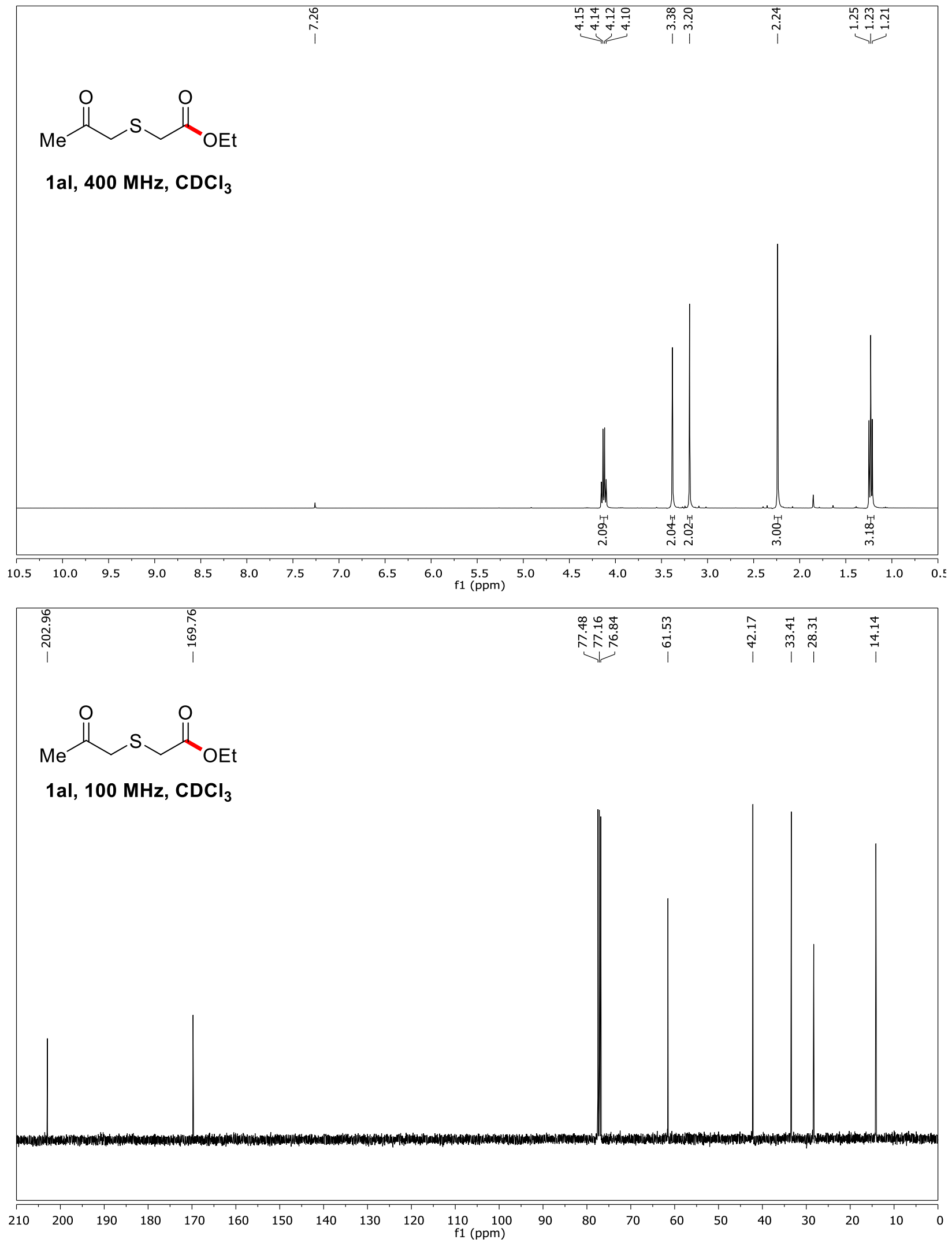
10. ${ }^{1} \mathrm{H}$ and ${ }^{13} \mathrm{C}$ NMR Spectra of Functionalized Enol Ethers

Ethyl-2-(phenylthio)-2-((1-phenylvinyl)oxy)acetate (3a)
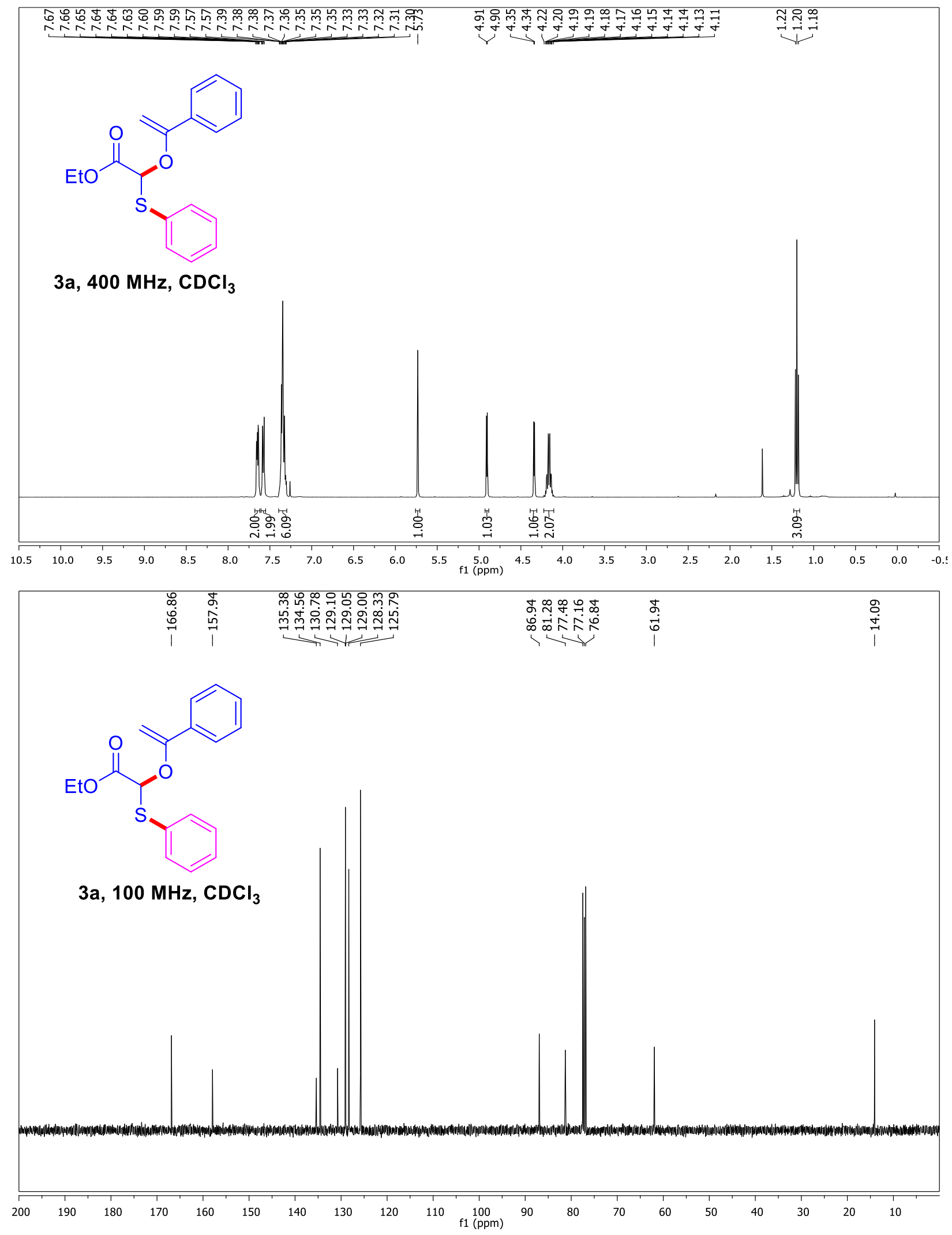
Ethyl-2-((1-(4-methoxyphenyl)vinyl)oxy)-2-(phenylthio)acetate (3b)
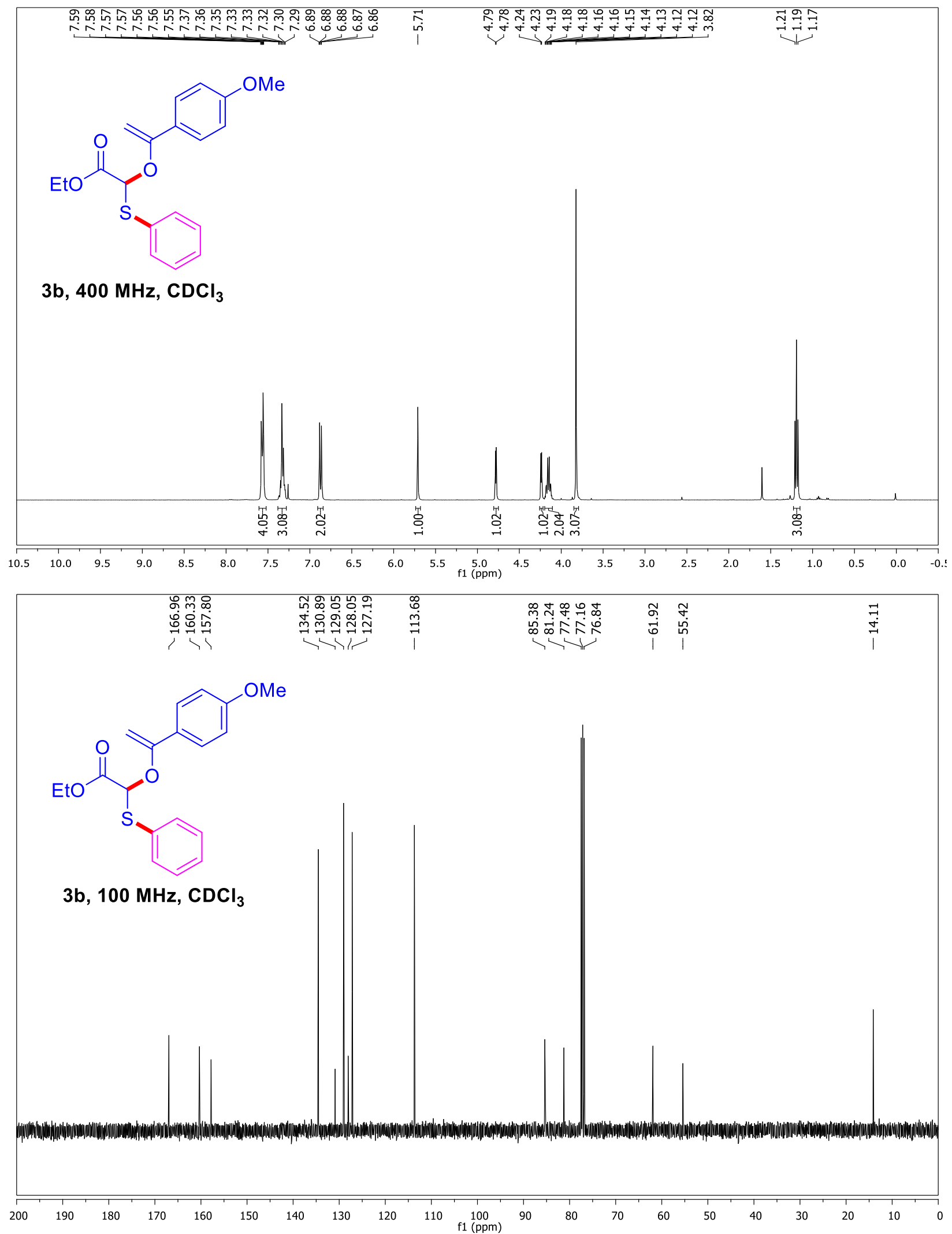
Ethyl 2-(phenylthio)-2-((1-(p-tolyl)vinyl)oxy)acetate (3c)
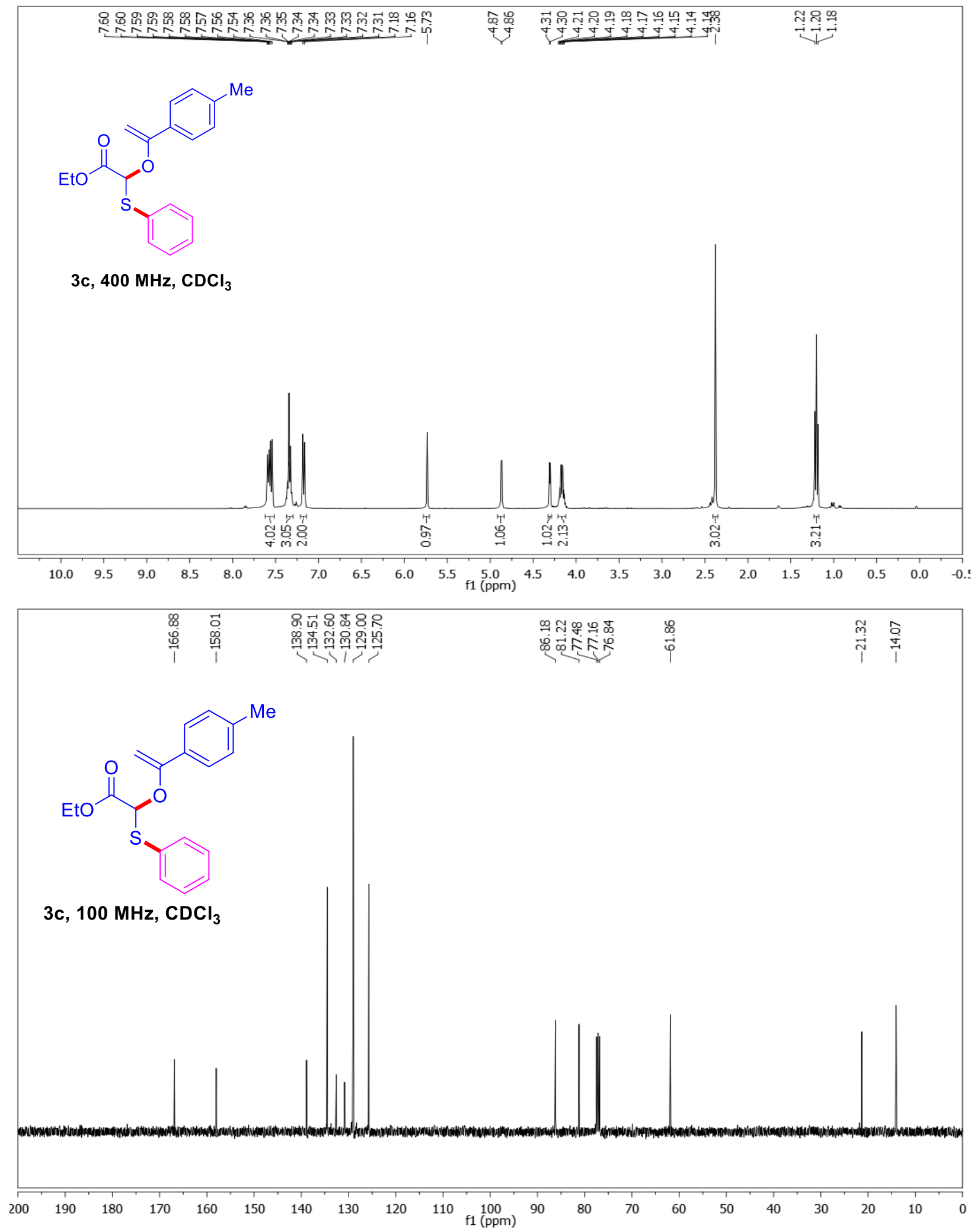
Ethyl-2-((1-(4-bromophenyl)vinyl)oxy)-2-(phenylthio)acetate (3d)
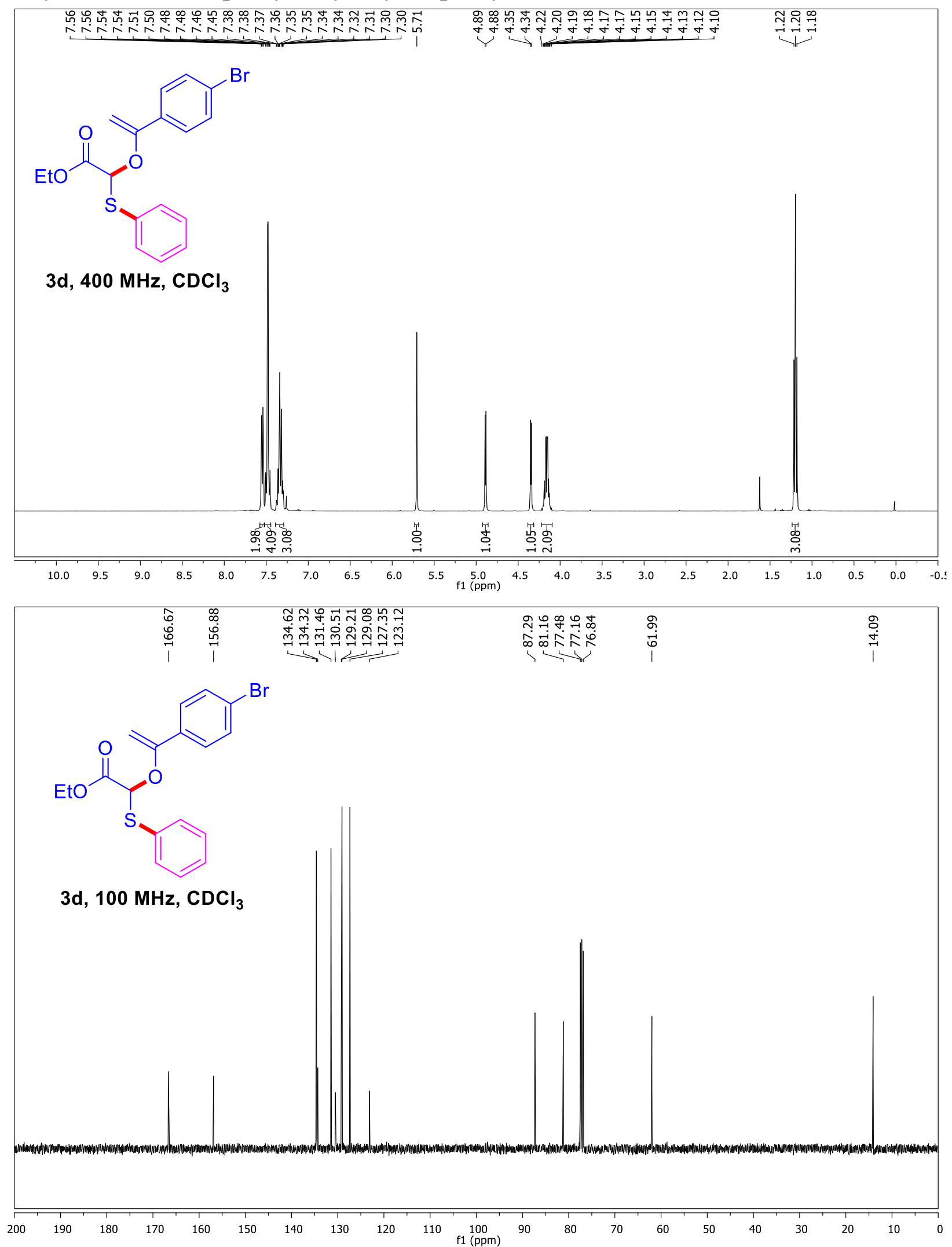
Ethyl-2-((1-(4-chlorophenyl)vinyl)oxy)-2-(phenylthio)acetate (3e)
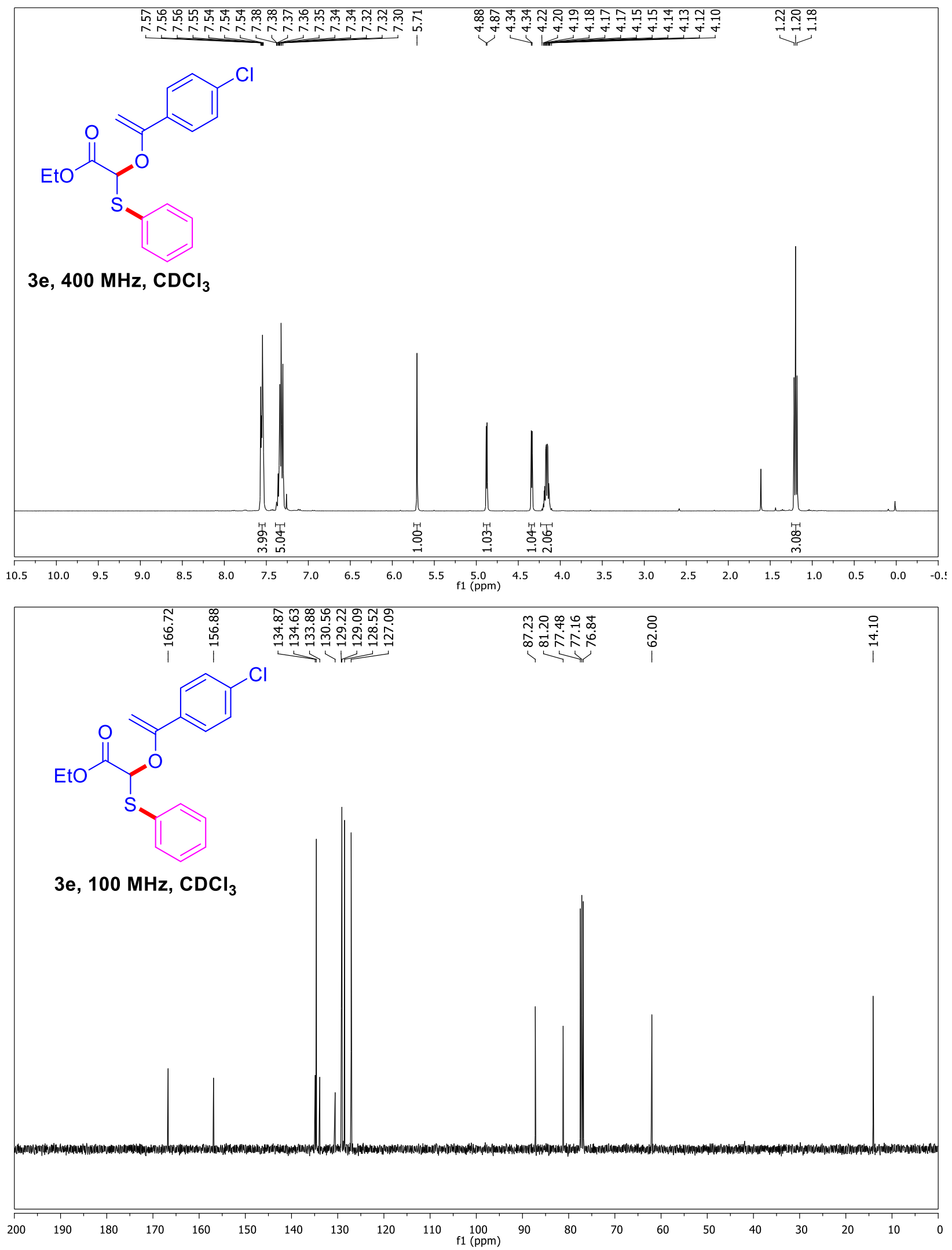
Ethyl-2-((1-(4-fluorophenyl)vinyl)oxy)-2-(phenylthio)acetate (3f)
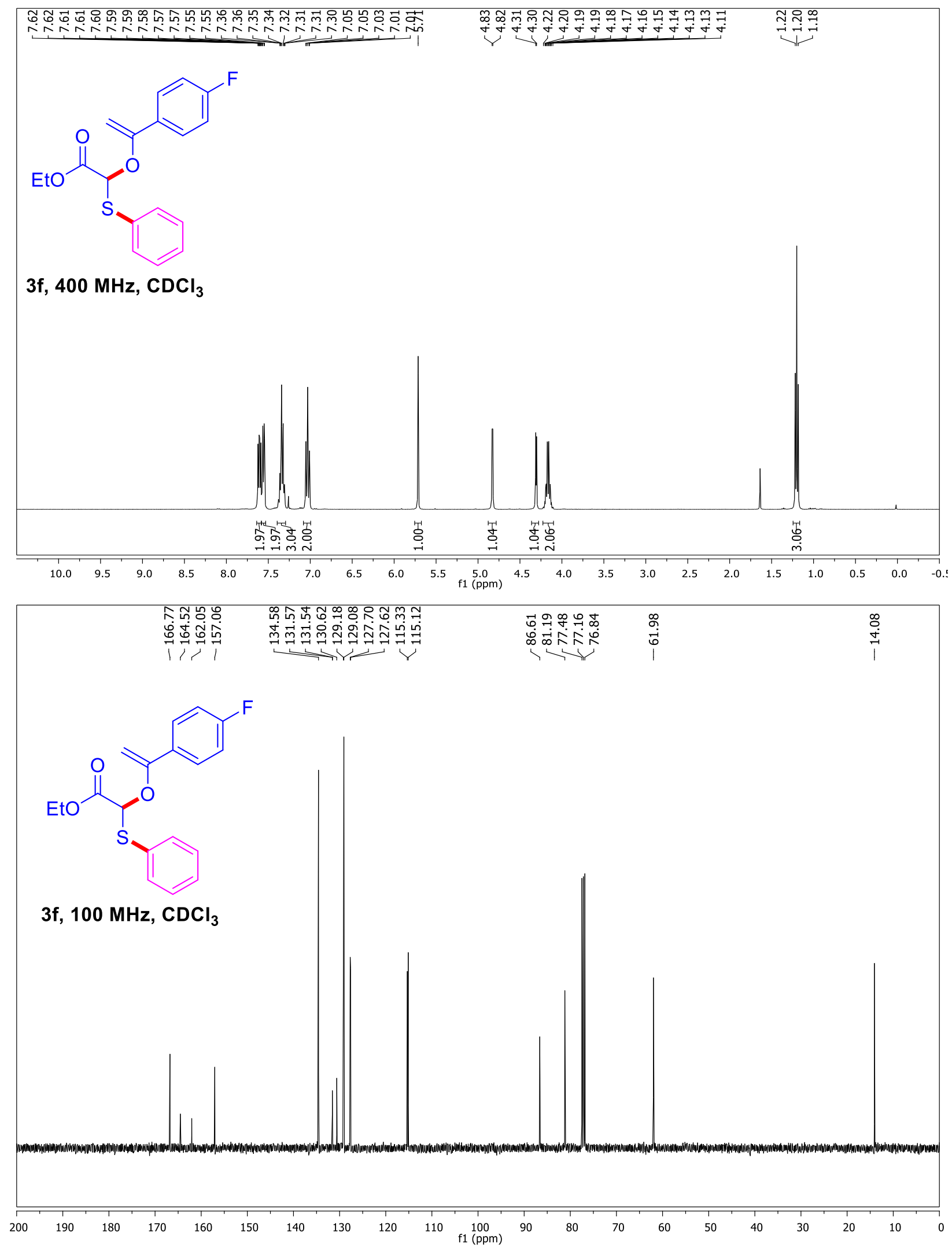
Ethyl-2-((1-(4-cyanophenyl)vinyl)oxy)-2-(phenylthio)acetate (3g)
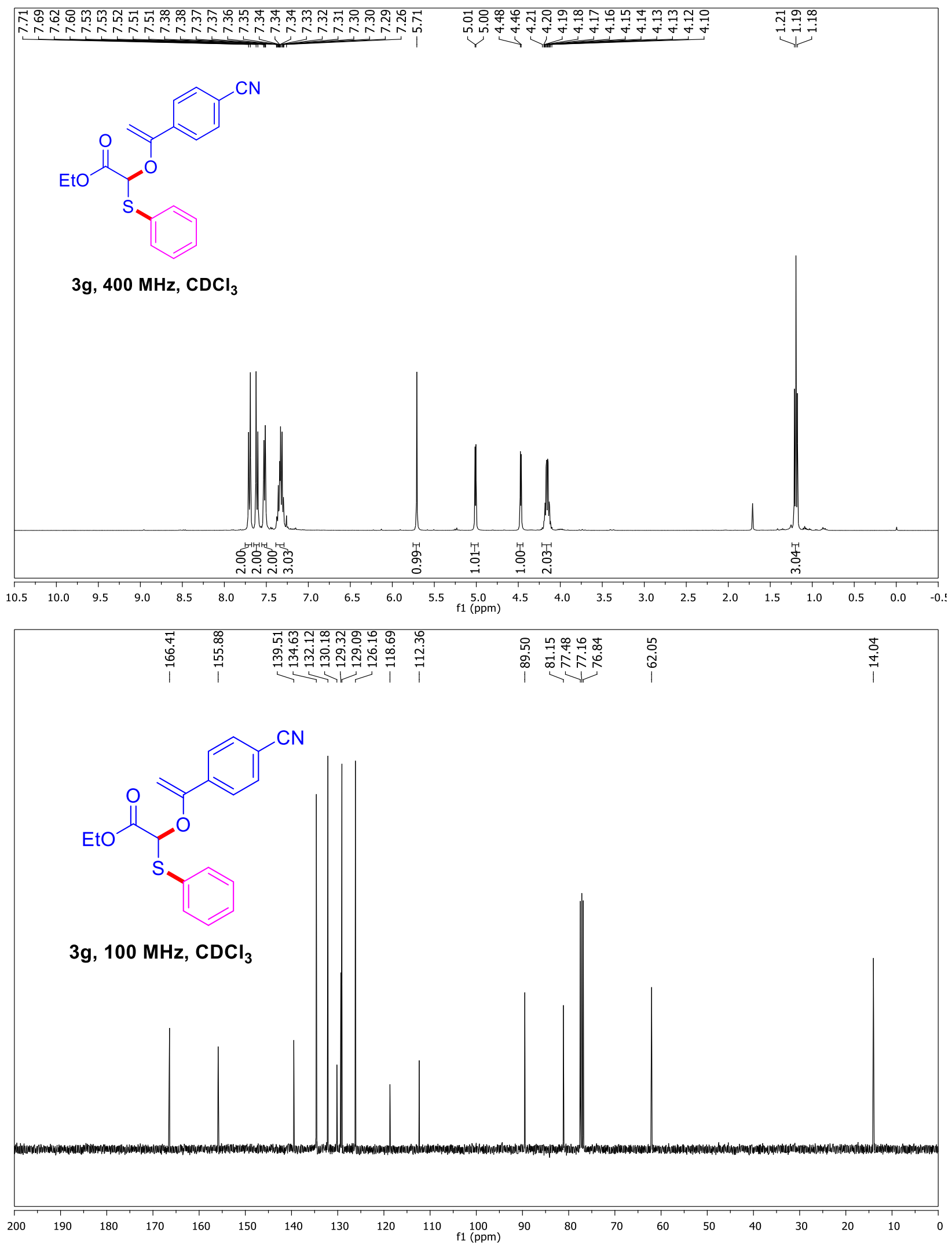
Ethyl-2-((1-(4-nitrophenyl)vinyl)oxy)-2-(phenylthio)acetate (3h)
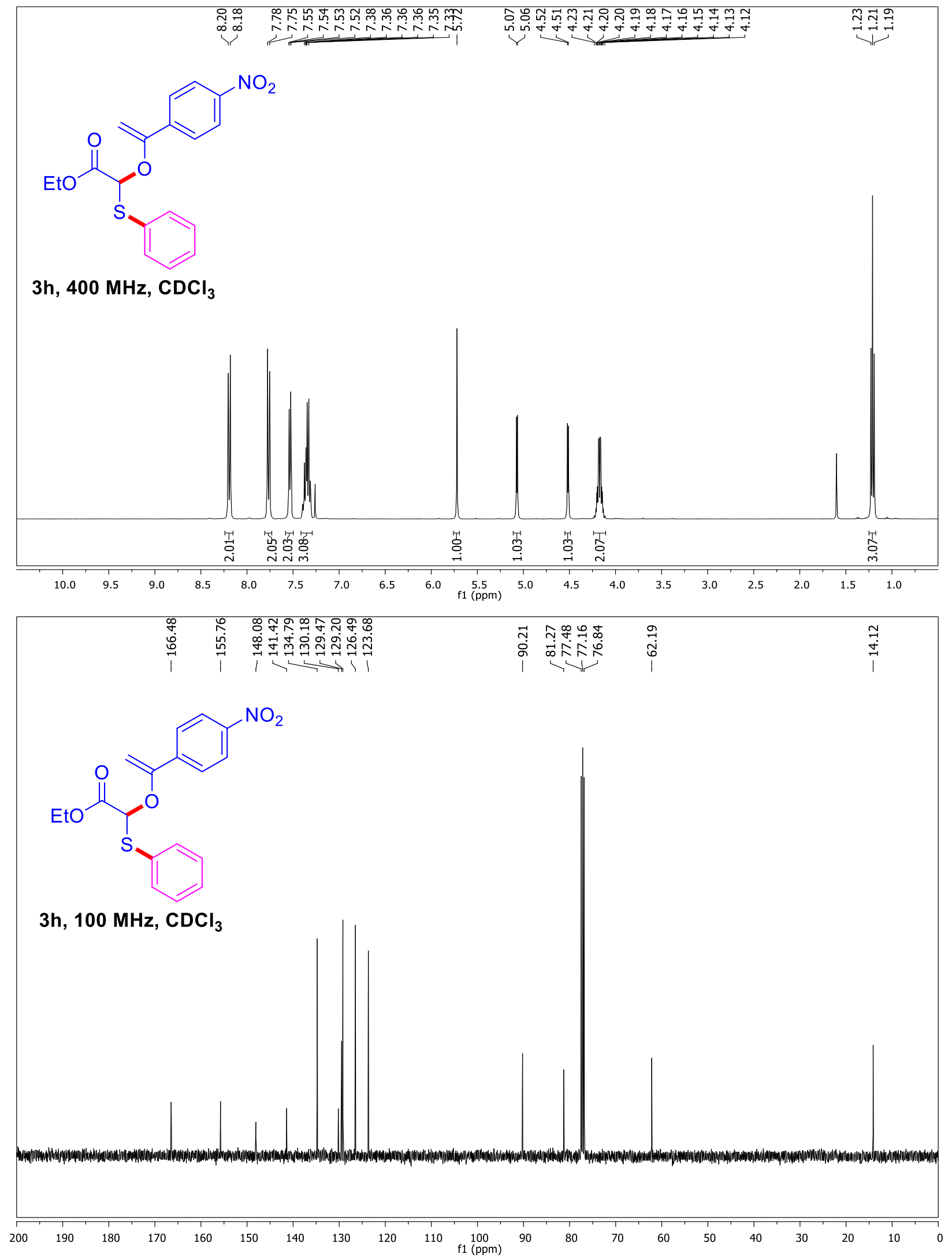
Ethyl-2-((1-(3-bromophenyl)vinyl)oxy)-2-(phenylthio)acetate (3i)
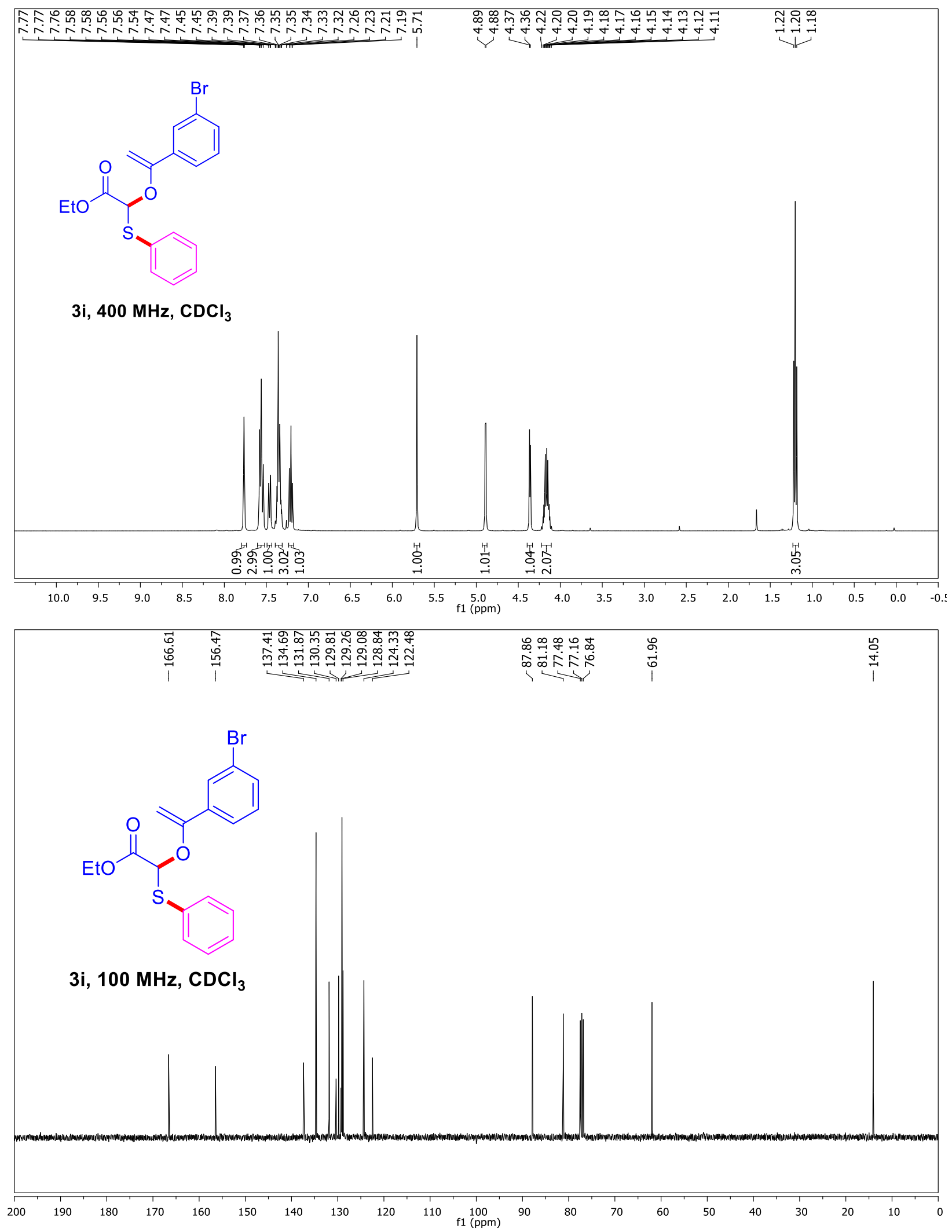
Ethyl-2-((1-(3-chlorophenyl)vinyl)oxy)-2-(phenylthio)acetate (3j)
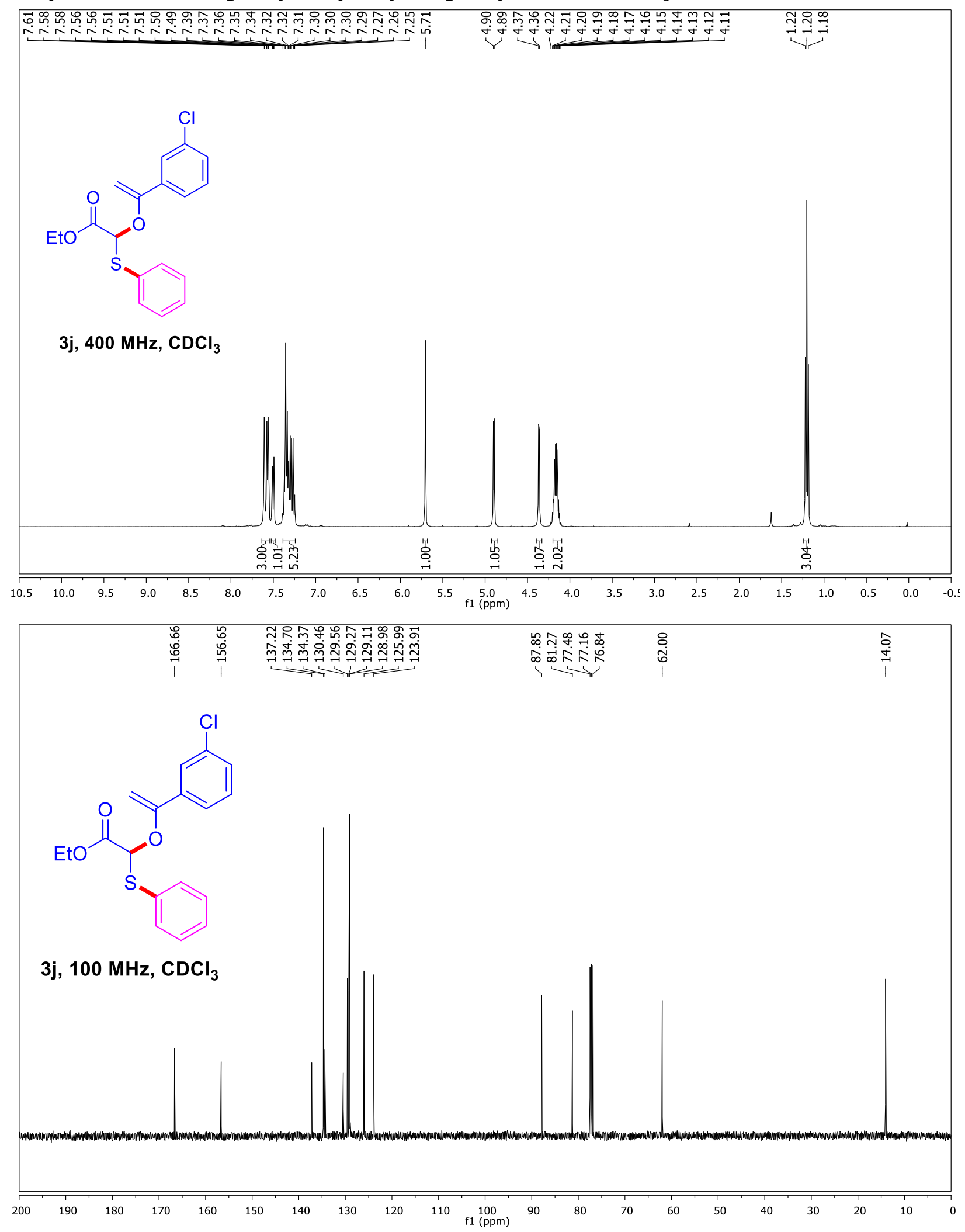
Ethyl-2-((1-(3-nitrophenyl)vinyl)oxy)-2-(phenylthio)acetate (3k)
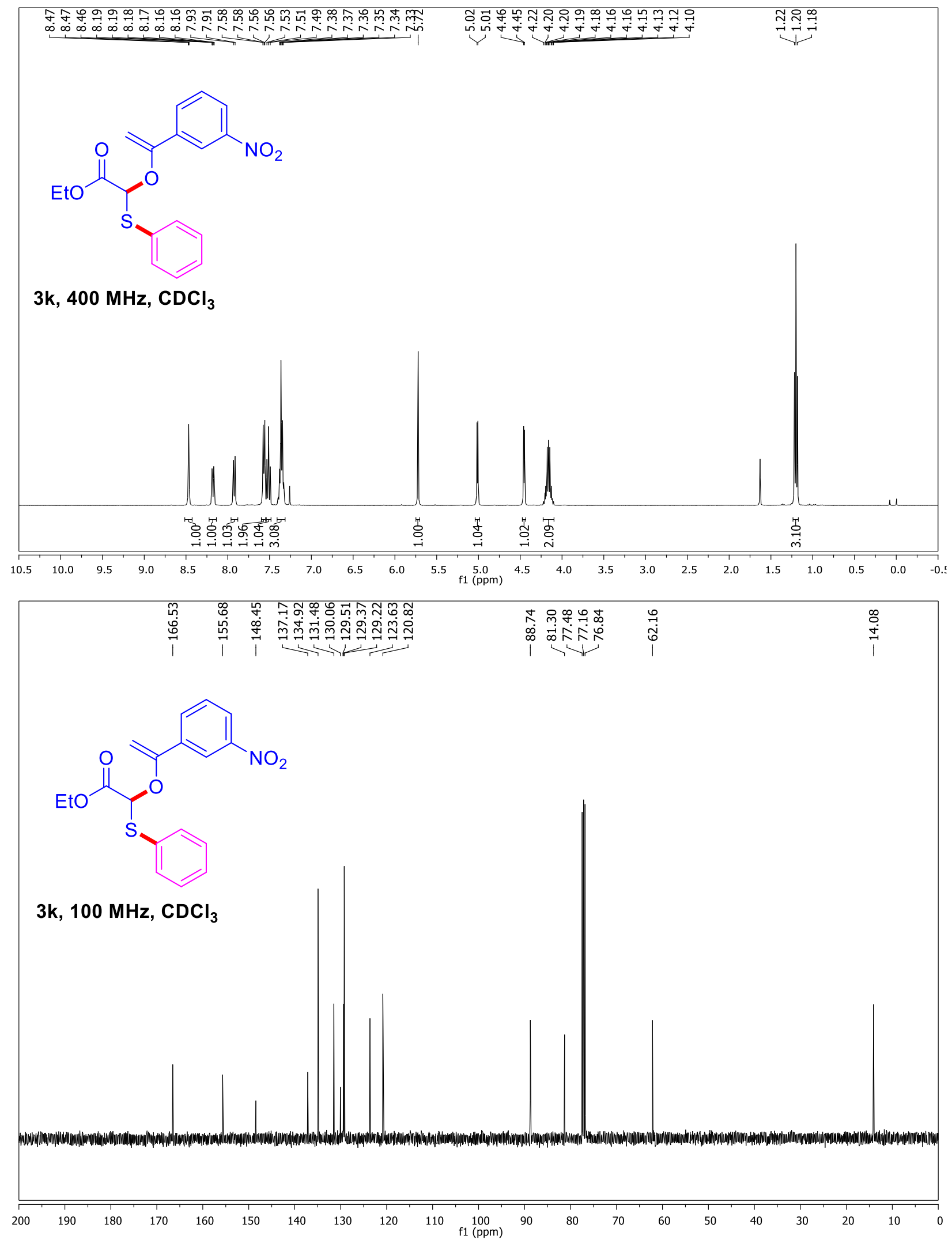
Ethyl-2-((1-(2-methoxyphenyl)vinyl)oxy)-2-(phenylthio)acetate (31)
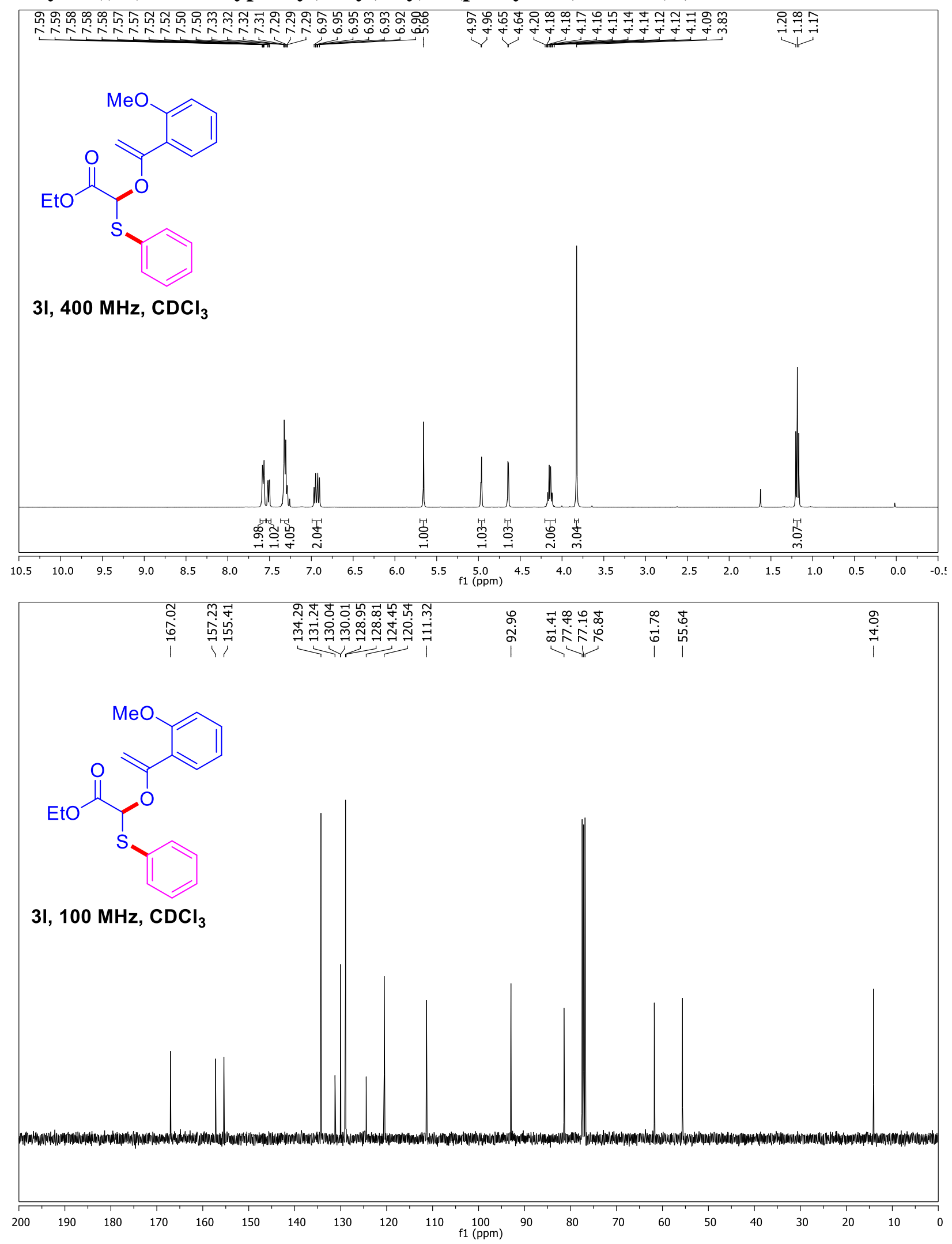
Ethyl-2-((1-(2-chlorophenyl)vinyl)oxy)-2-(phenylthio)acetate (3m)
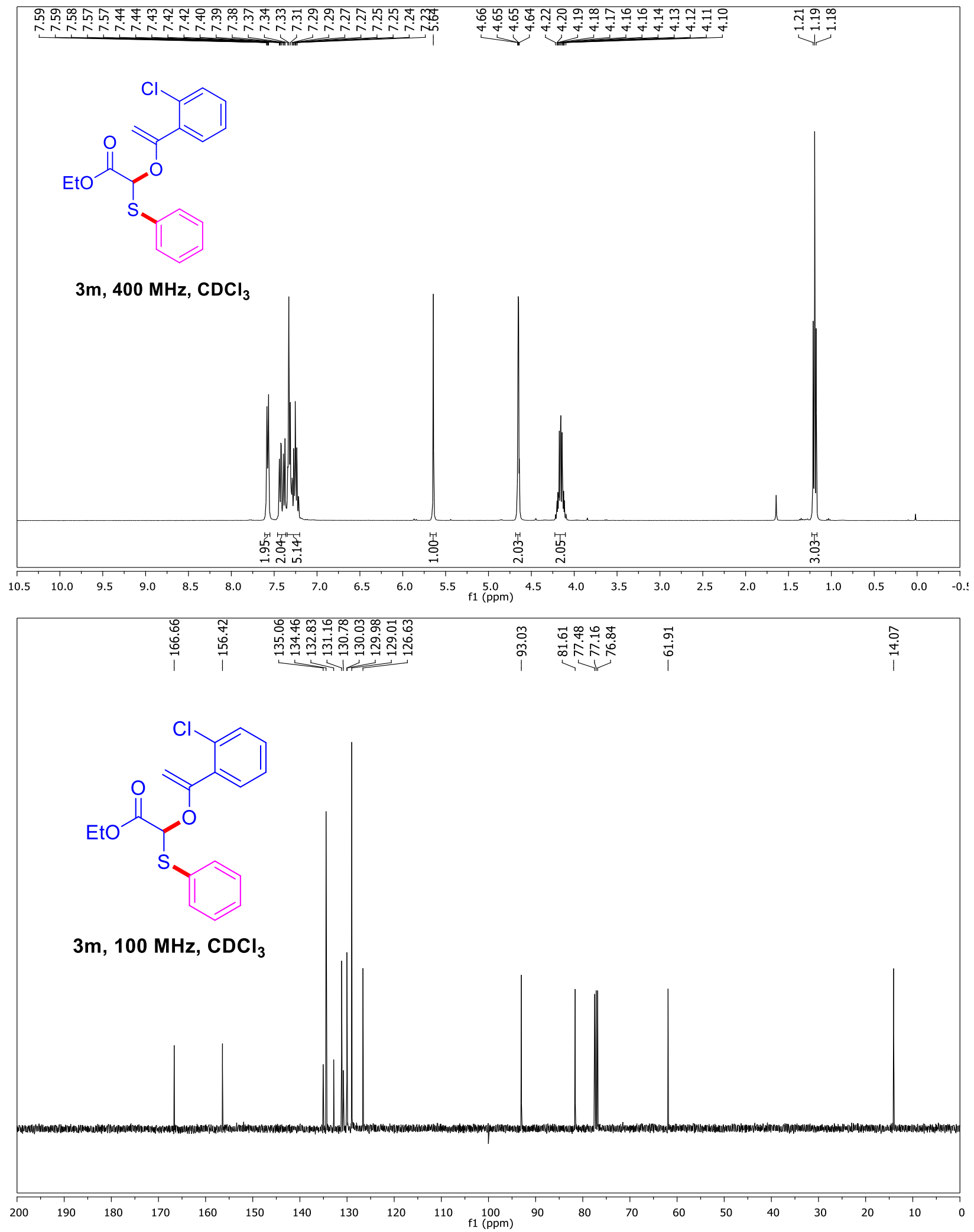
Ethyl-2-((1-(2-nitrophenyl)vinyl)oxy)-2-(phenylthio)acetate (3n)
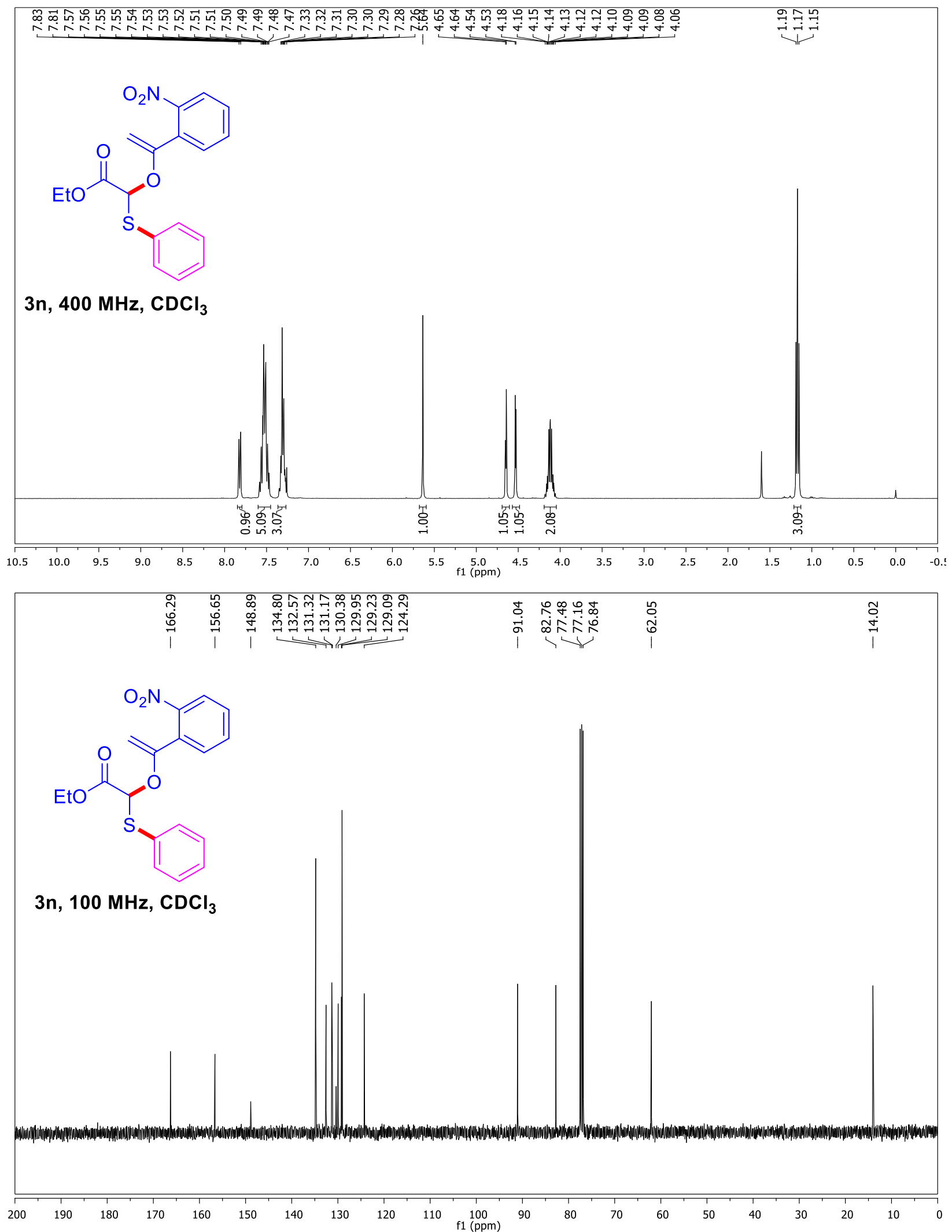
Ethyl-2-((1-(3,4-dimethoxyphenyl)vinyl)oxy)-2-(phenylthio)acetate (30)
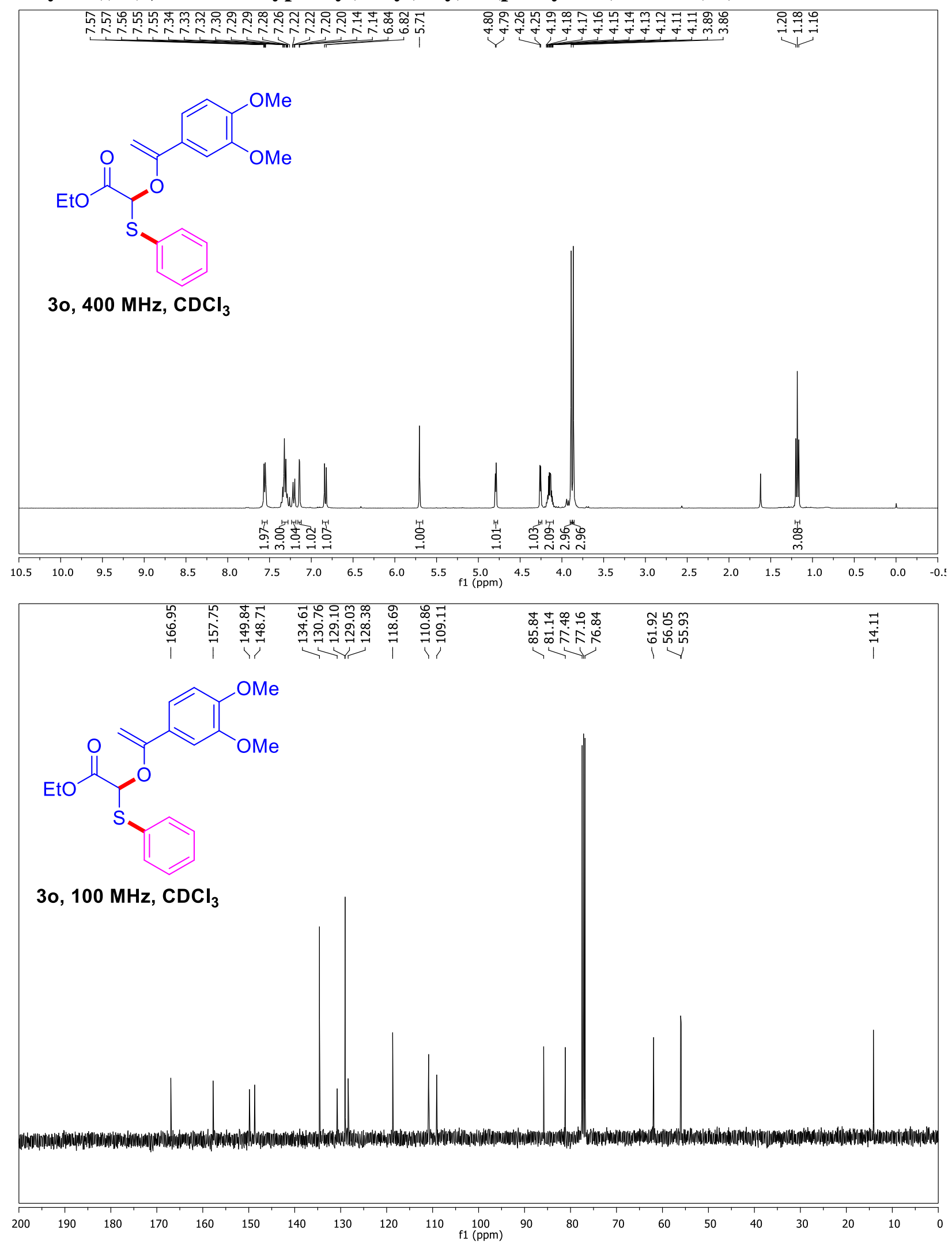
Ethyl-2-((1-(3-bromo-4-fluorophenyl)vinyl)oxy)-2-(phenylthio)acetate (3p)
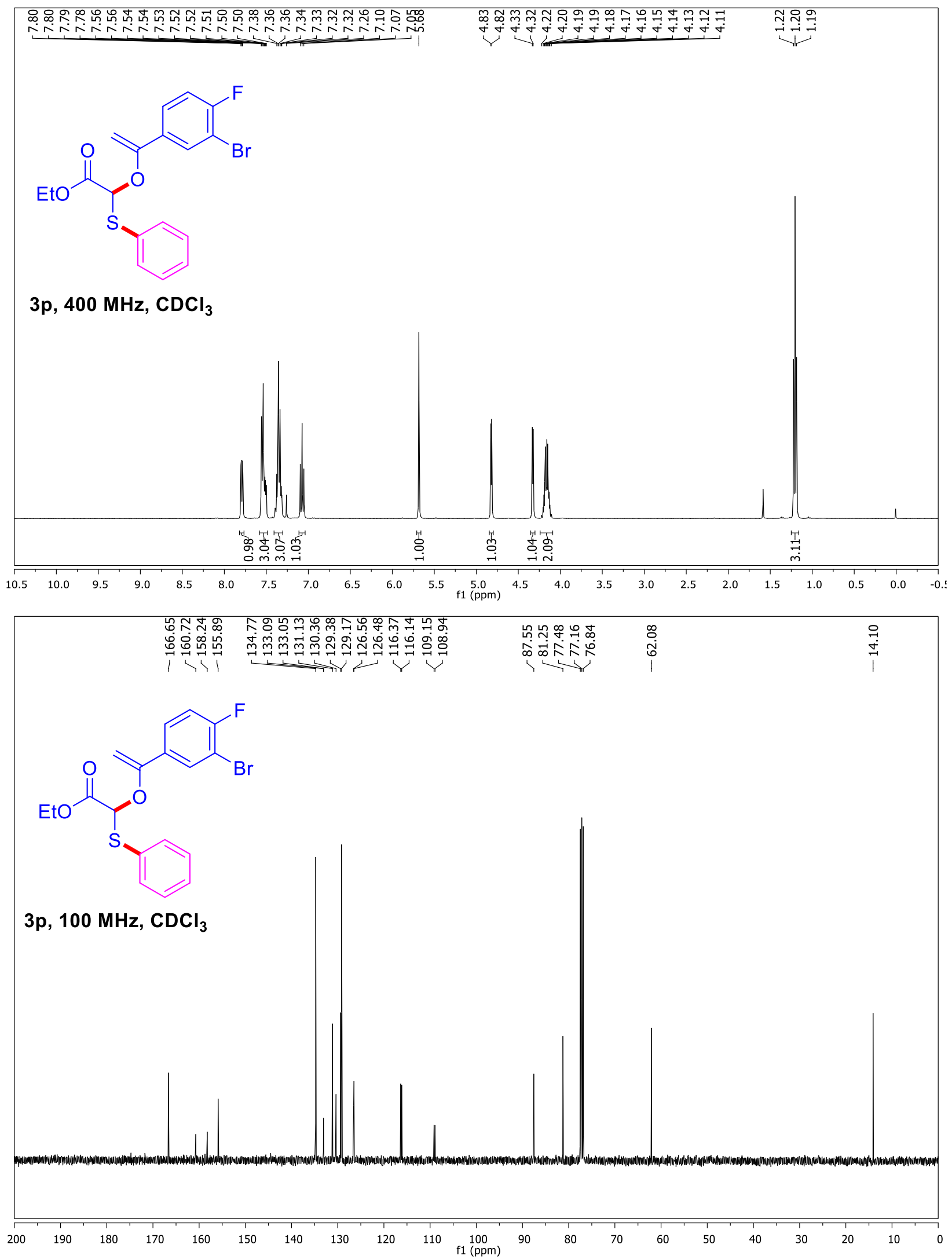
Ethyl-2-((1-(naphthalen-2-yl)vinyl)oxy)-2-(phenylthio)acetate (3q)
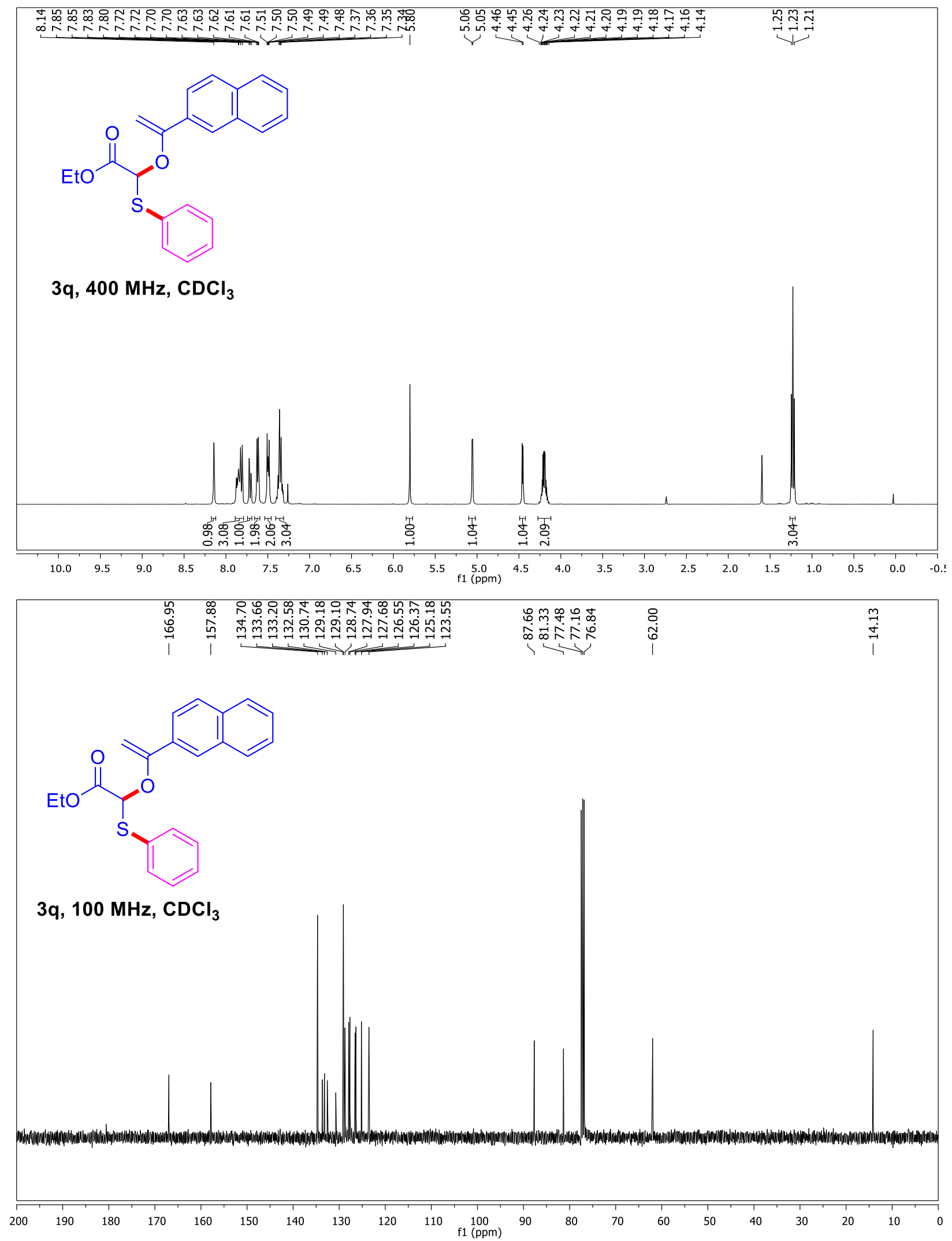
Ethyl-2-((1-(naphthalen-1-yl)vinyl)oxy)-2-(phenylthio)acetate (3r)
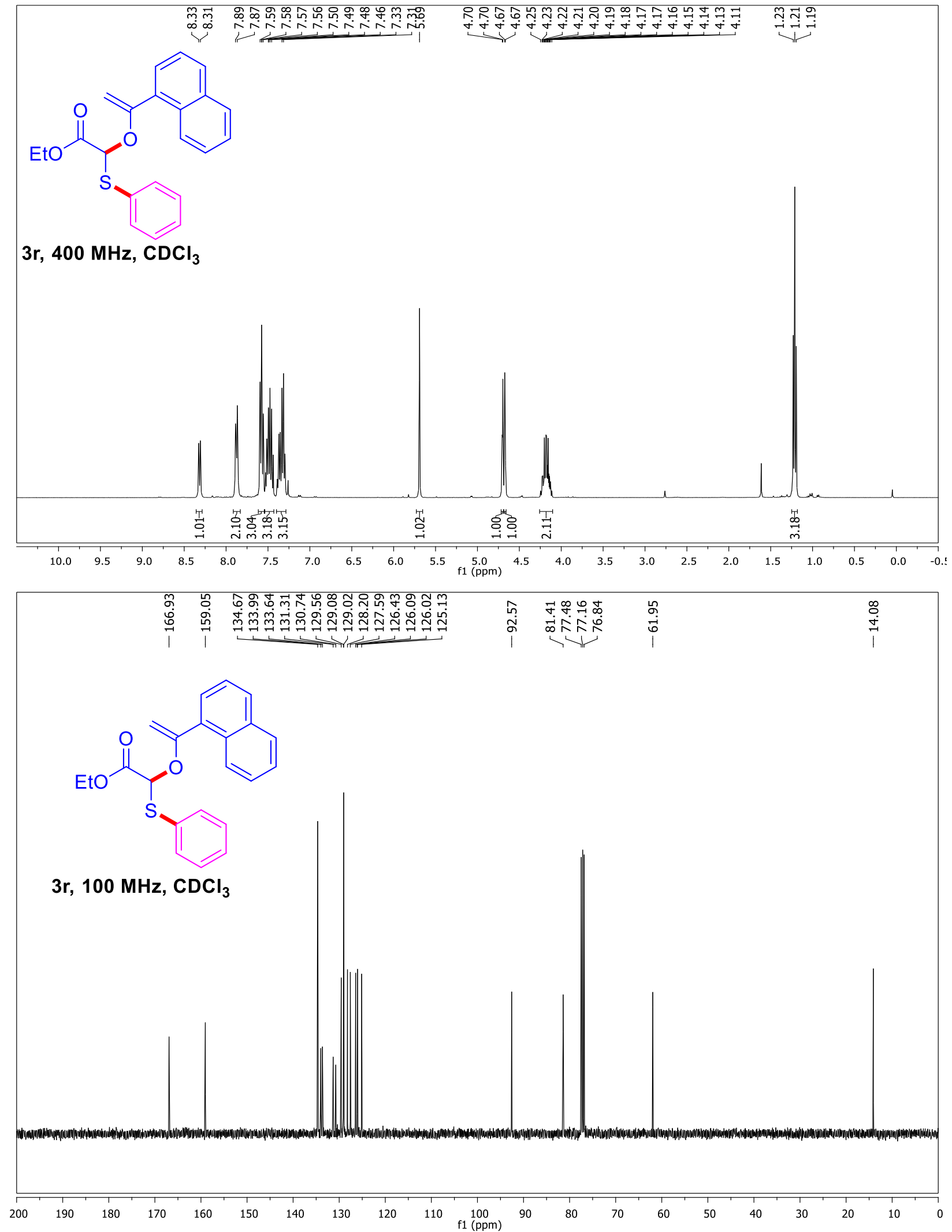
Ethyl-2-((1-(3,5-difluorophenyl)vinyl)oxy)-2-(phenylthio)acetate (3s)
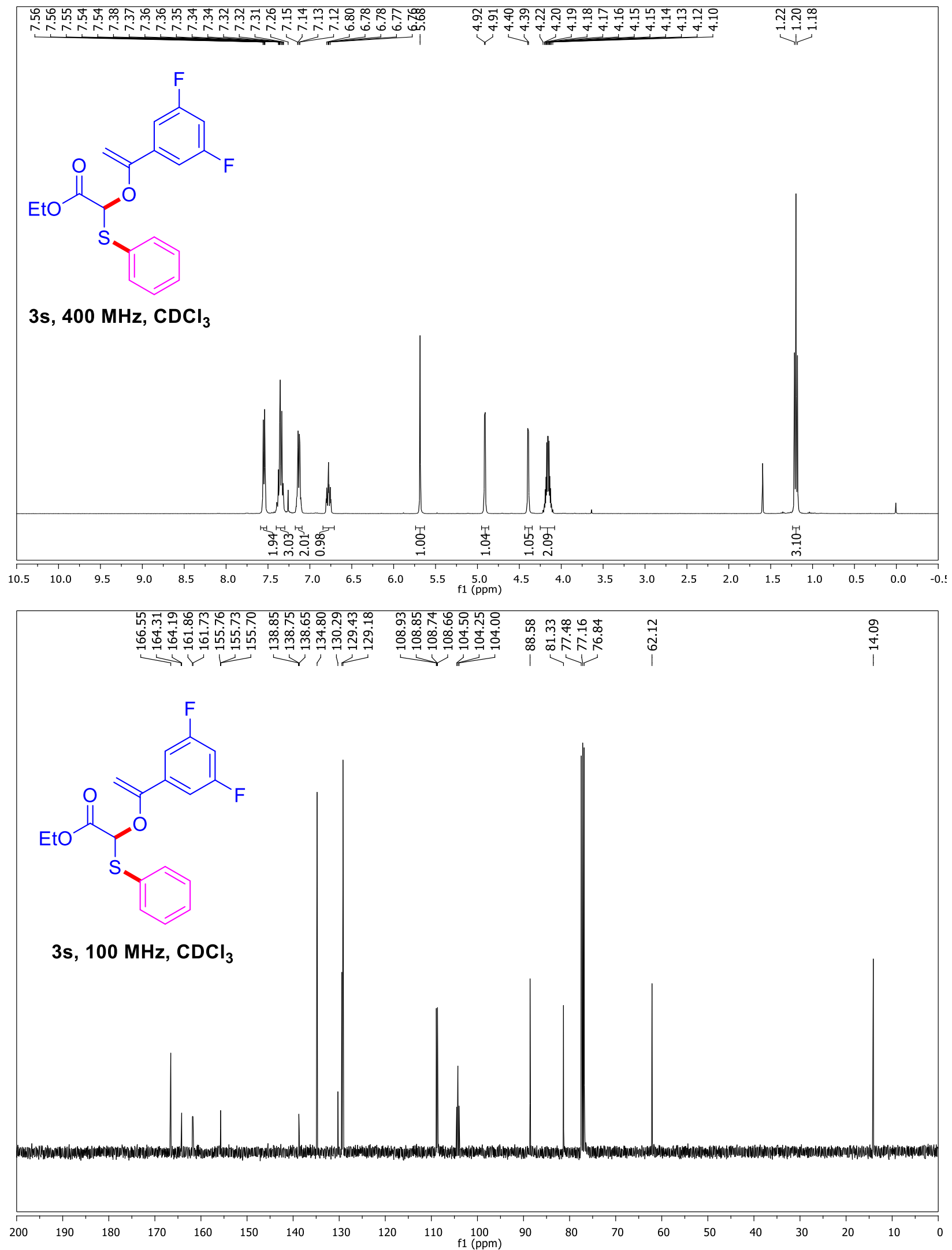
Ethyl-2-(phenylthio)-2-((1-(thiophen-2-yl)vinyl)oxy)acetate (3t)
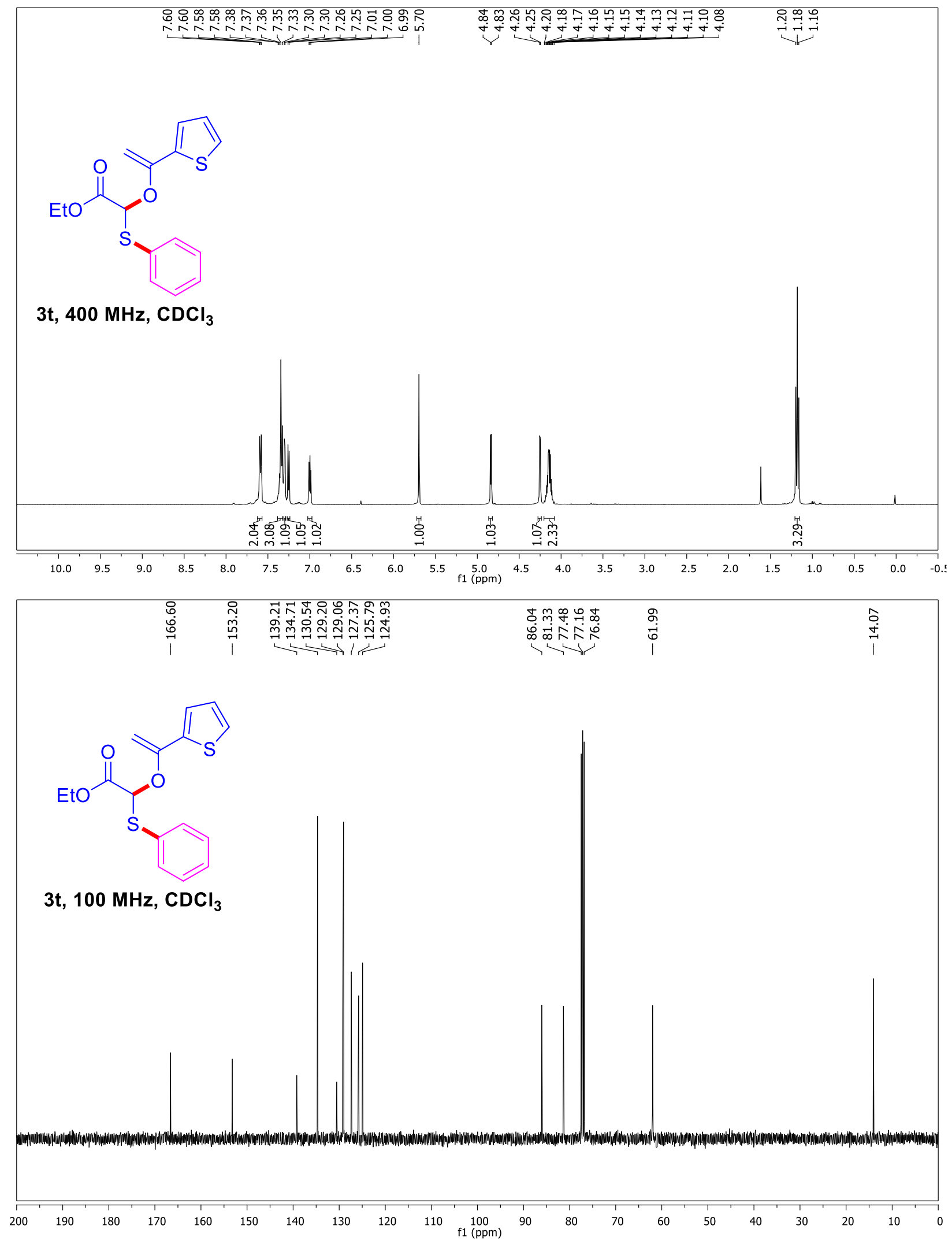
Methyl-2-(phenylthio)-2-((1-phenylvinyl)oxy)acetate (3u)
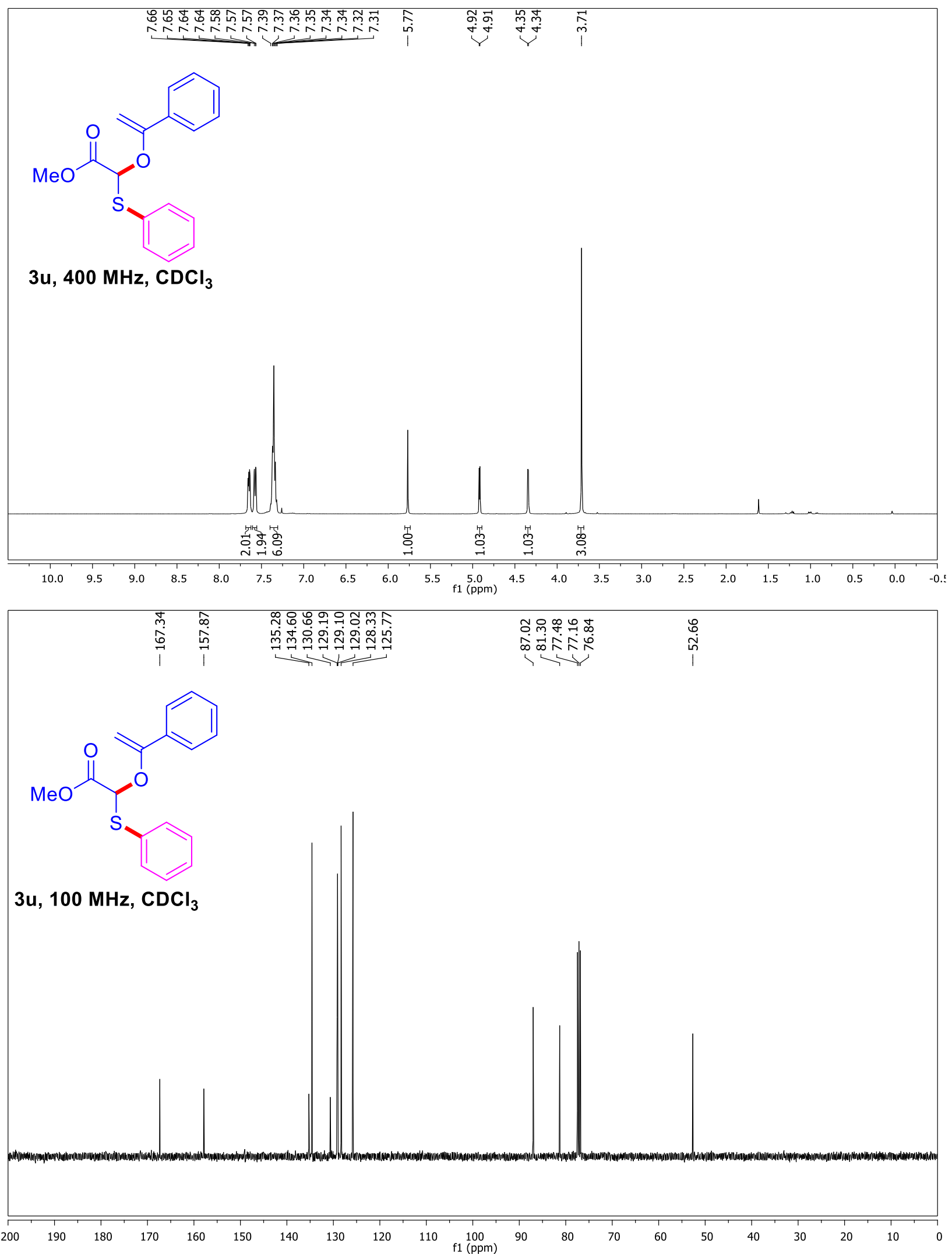
Butyl-2-(phenylthio)-2-((1-phenylvinyl)oxy)acetate (3v)

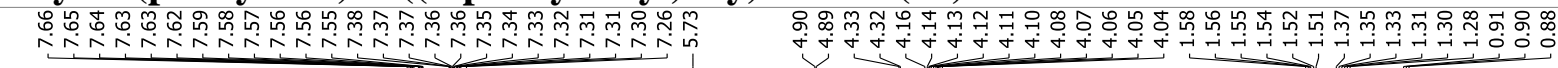<smiles>C=C(OC(SC/C=C/C)C(=O)OCCCC)c1ccccc1</smiles>

3v, $400 \mathrm{MHz}, \mathrm{CDCl}_{3}$
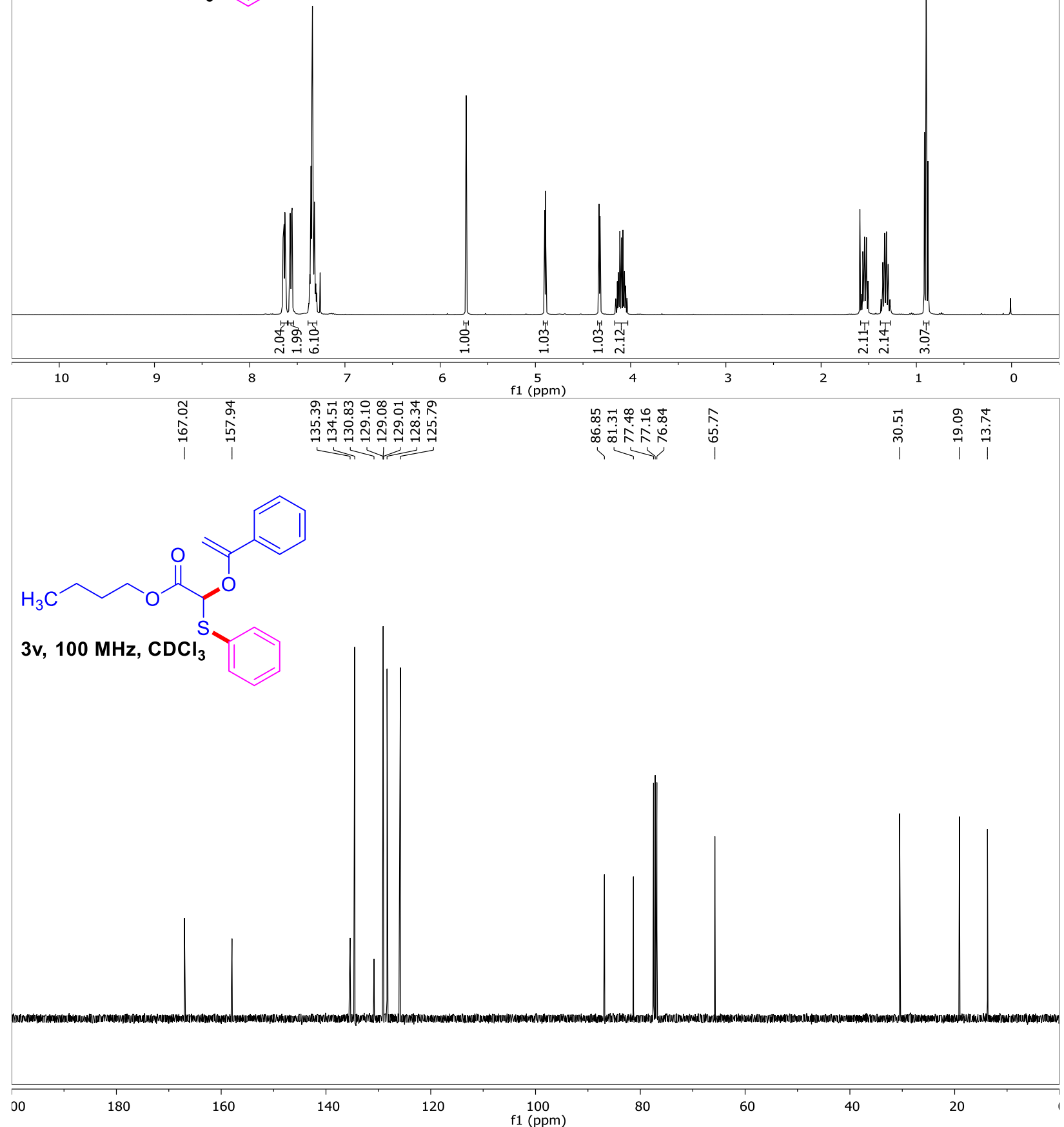
Isopropyl-2-(phenylthio)-2-((1-phenylvinyl)oxy)acetate (3w)
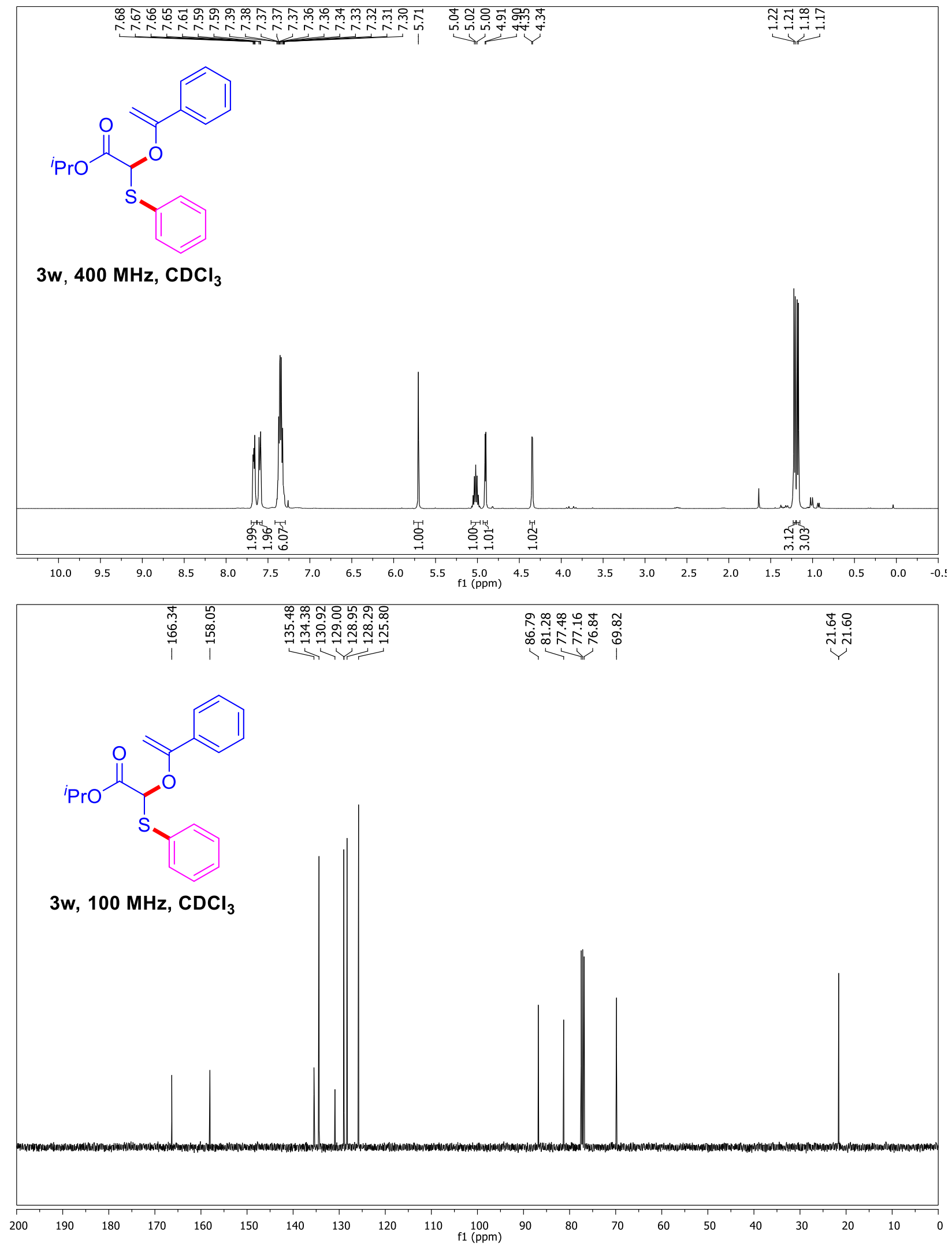
Allyl-2-(phenylthio)-2-((1-phenylvinyl)oxy)acetate (3x)
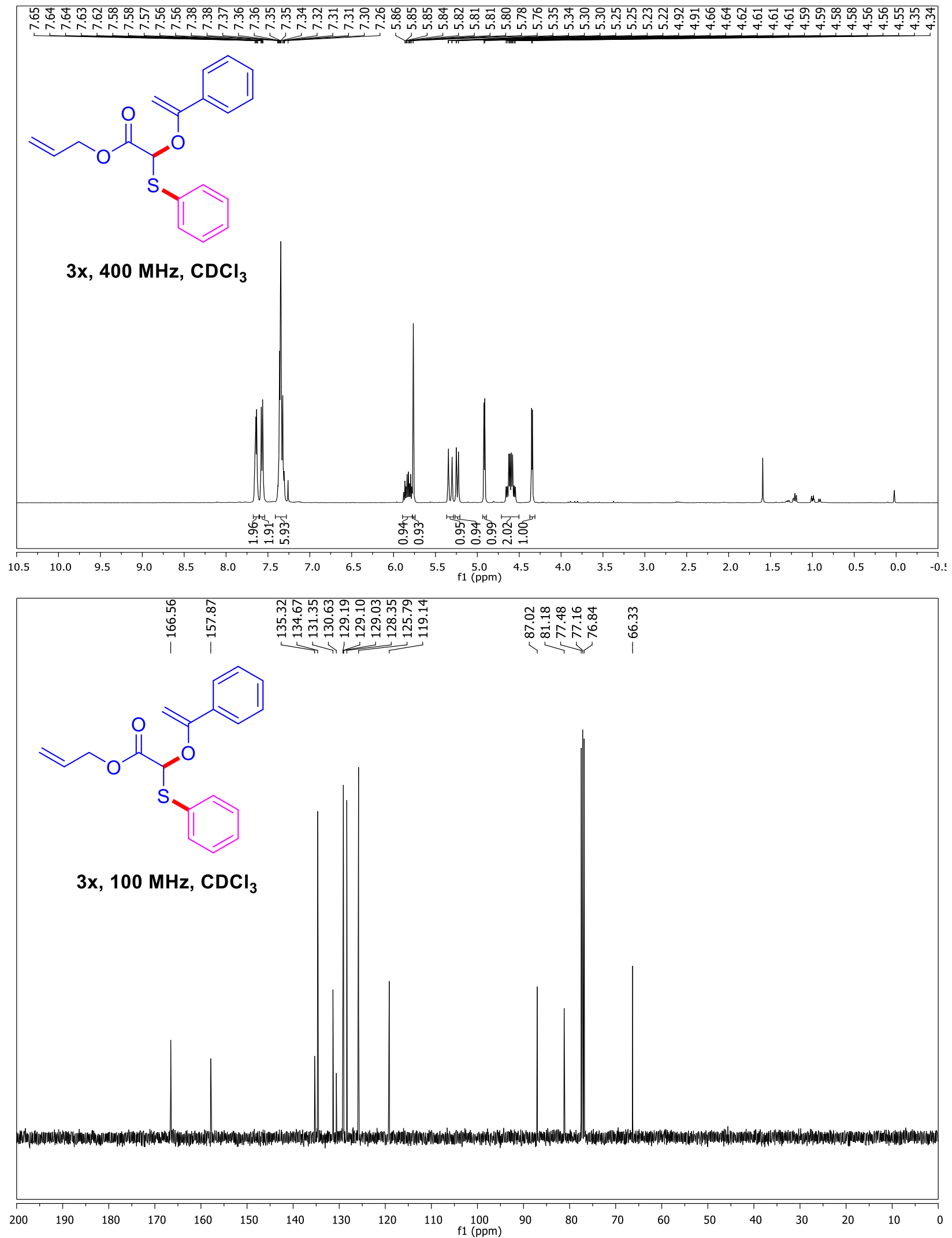
Prop-2-yn-1-yl-2-(phenylthio)-2-((1-phenylvinyl)oxy)acetate (3y)
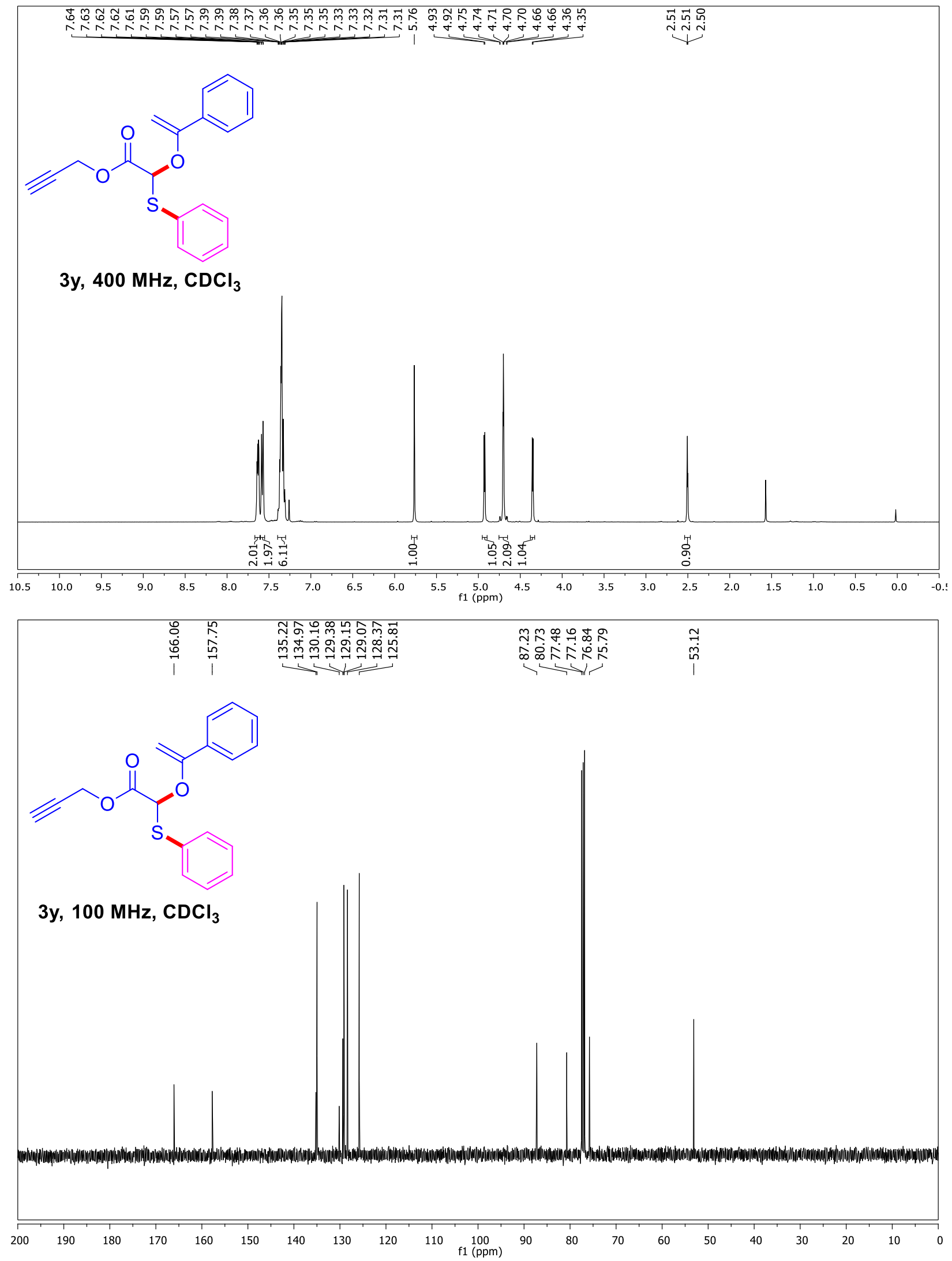
Phenethyl-2-(phenylthio)-2-((1-phenylvinyl)oxy)acetate (3z)
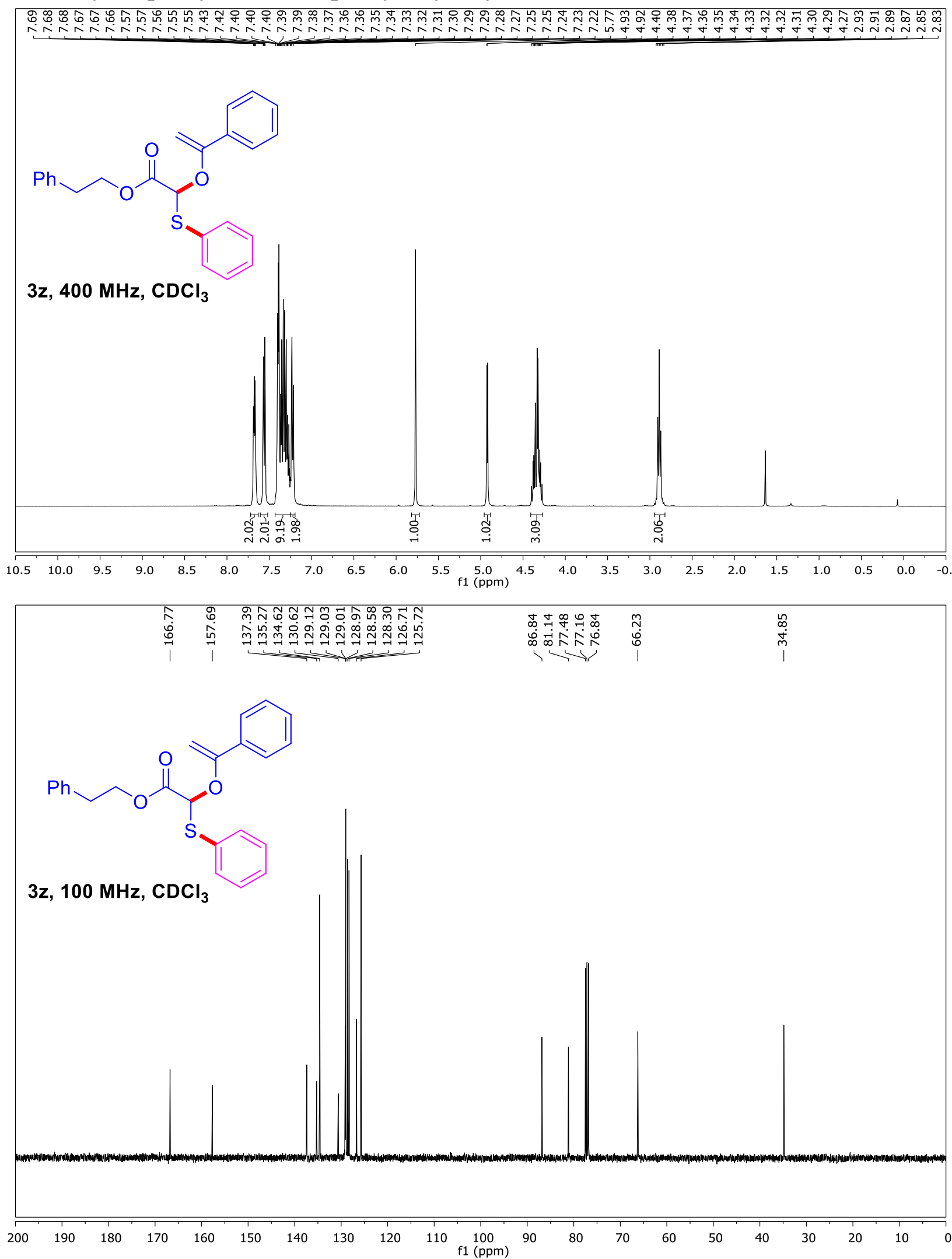
Phenyl-2-(phenylthio)-2-((1-phenylvinyl)oxy)acetate (3aa)
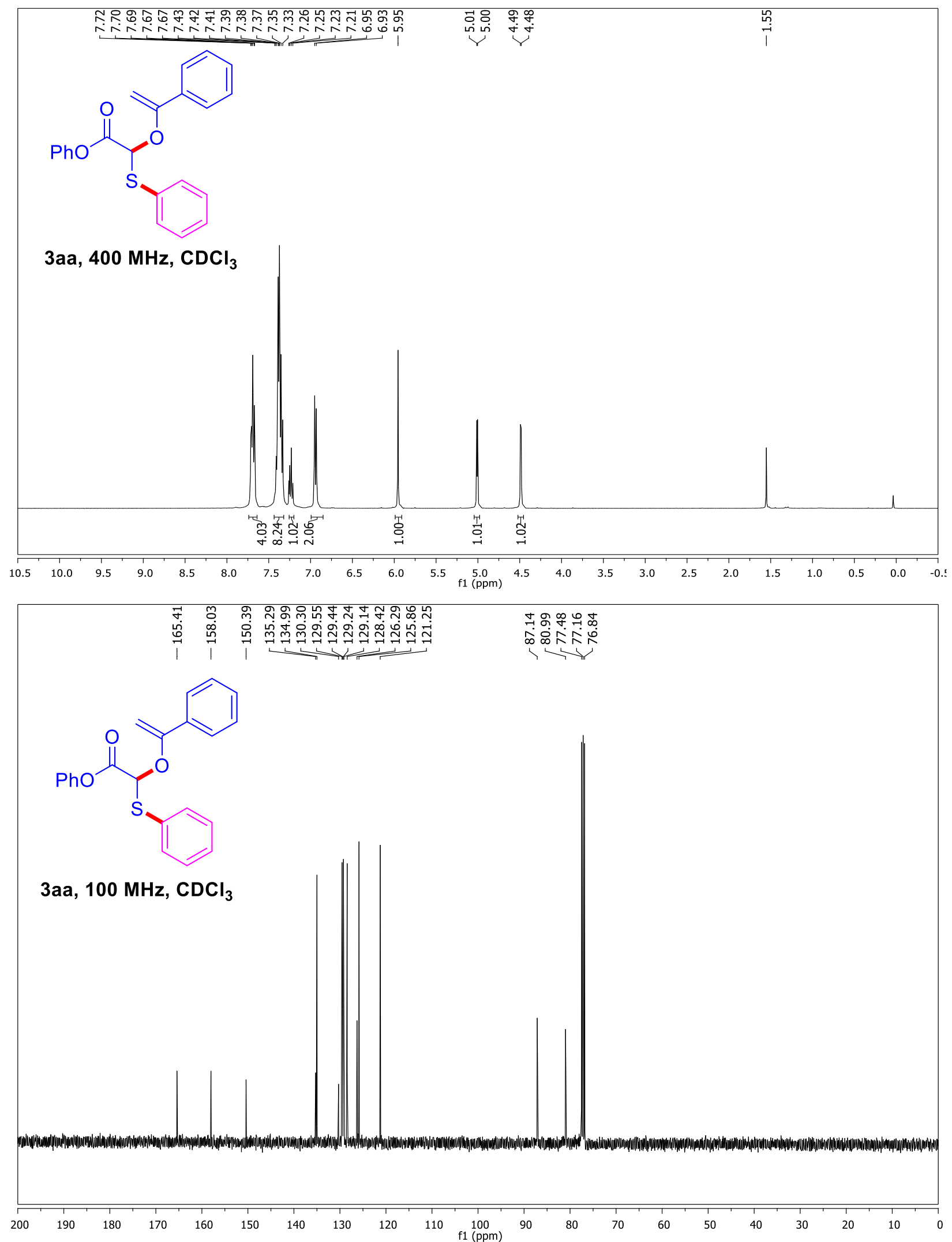
$(1 R, 2 S, 5 R)-2-$ Isopropyl-5-methylcyclohexyl-2-(phenylthio)-2-((1-phenylvinyl)oxy)acetate (3ab)
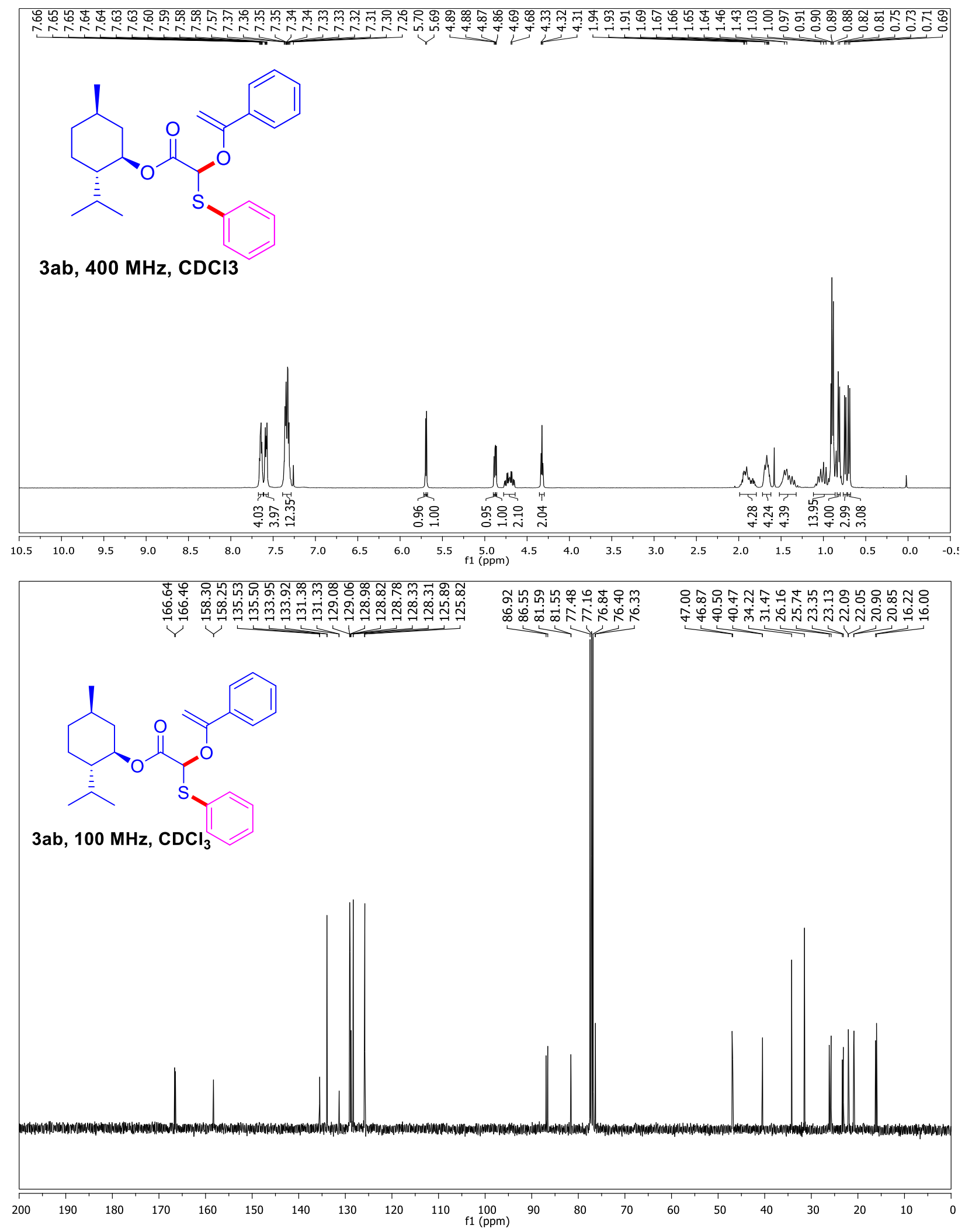
(E)-3,7-Dimethylocta-2,6-dien-1-yl-2-(phenylthio)-2-((1-phenylvinyl)oxy)acetate (3ac)
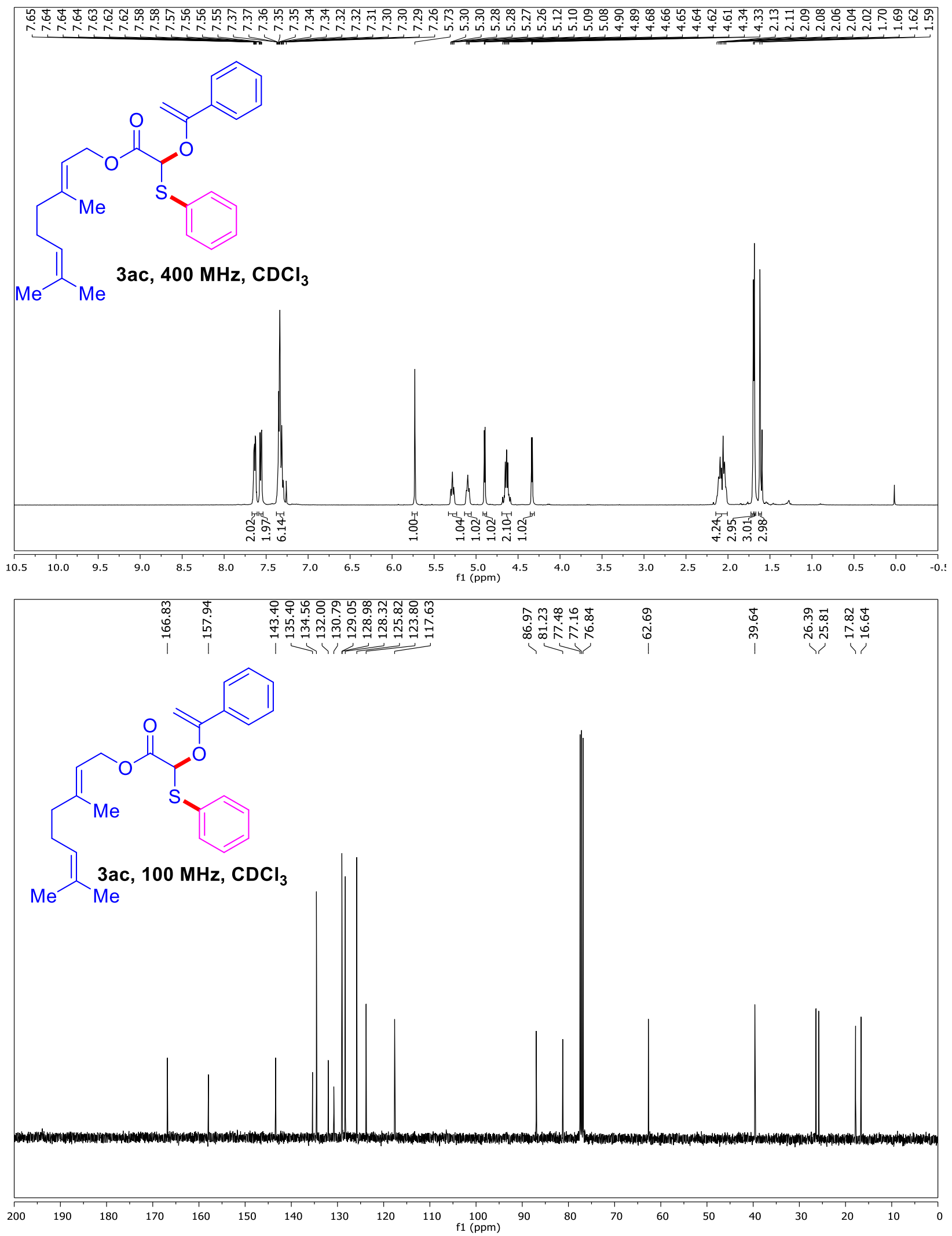
$(3 S, 8 S, 9 S, 10 R, 13 R, 14 S, 17 R)$-10,13-Dimethyl-17-((R)-6-methylheptan-2-yl)

2,3,4,7,8,9,10,11,12,13,14,15,16,17-tetradecahydro-1H-cyclopenta[a]phenanthren-3-yl

(phenylthio)-2-((1-phenylvinyl)oxy)acetate (3ad)
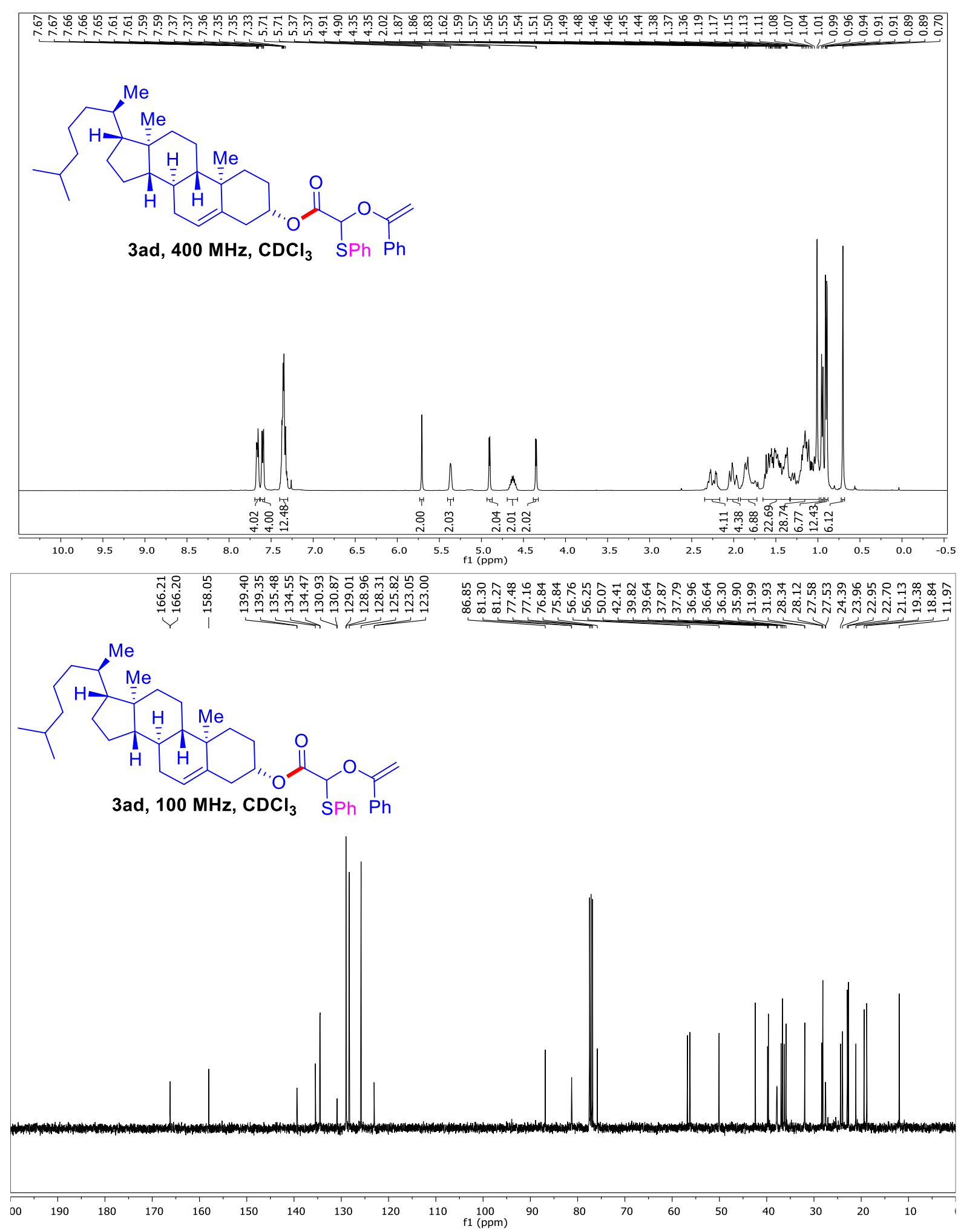
phenylvinyl)oxy)acetate (3ae)
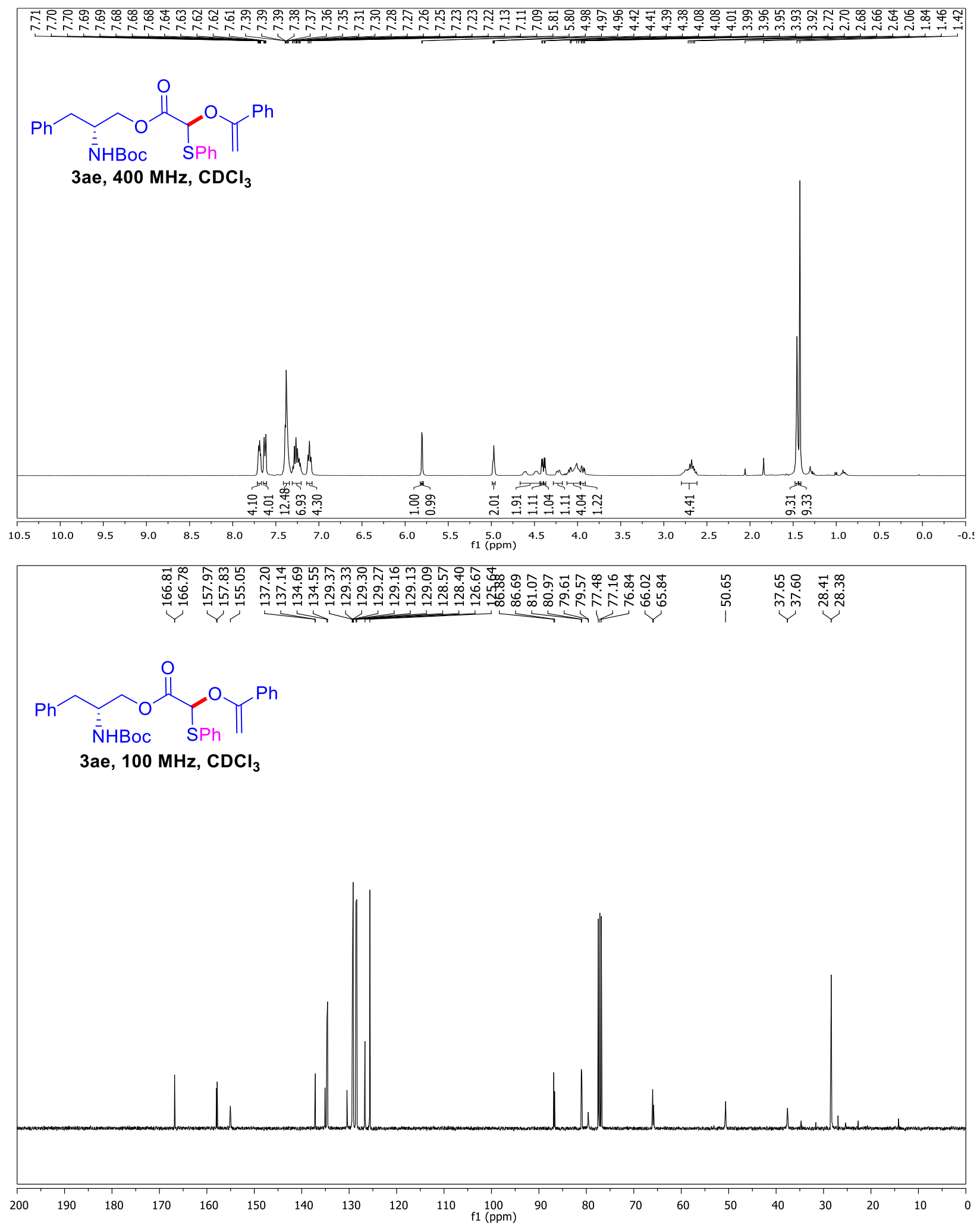
$\mathrm{N}, \mathrm{N}$-Dimethyl-2-(phenylthio)-2-((1-phenylvinyl)oxy)acetamide (3af)
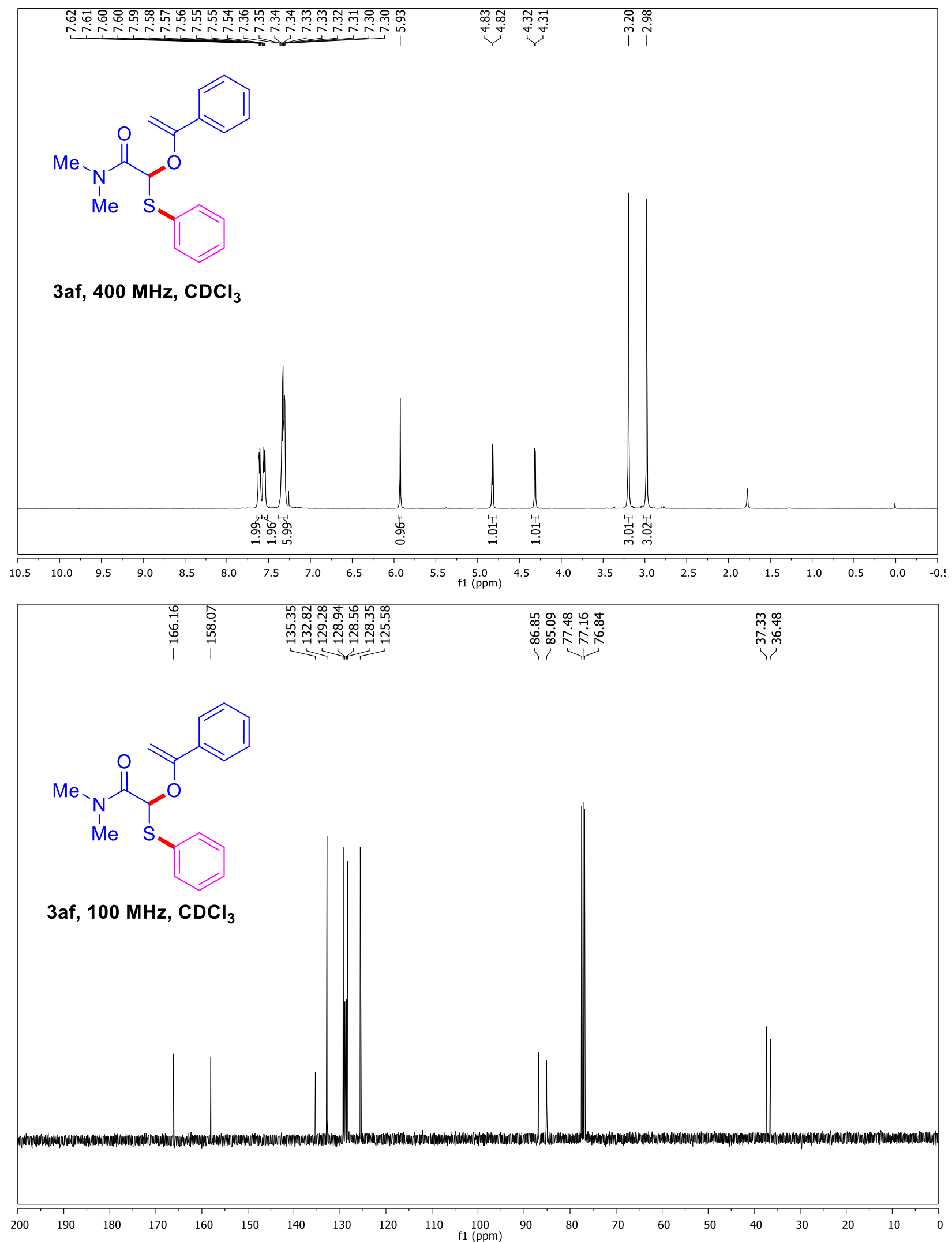
$\mathrm{N}$-Methoxy- $\mathrm{N}$-methyl-2-(phenylthio)-2-((1-phenylvinyl)oxy)acetamide (3ag)
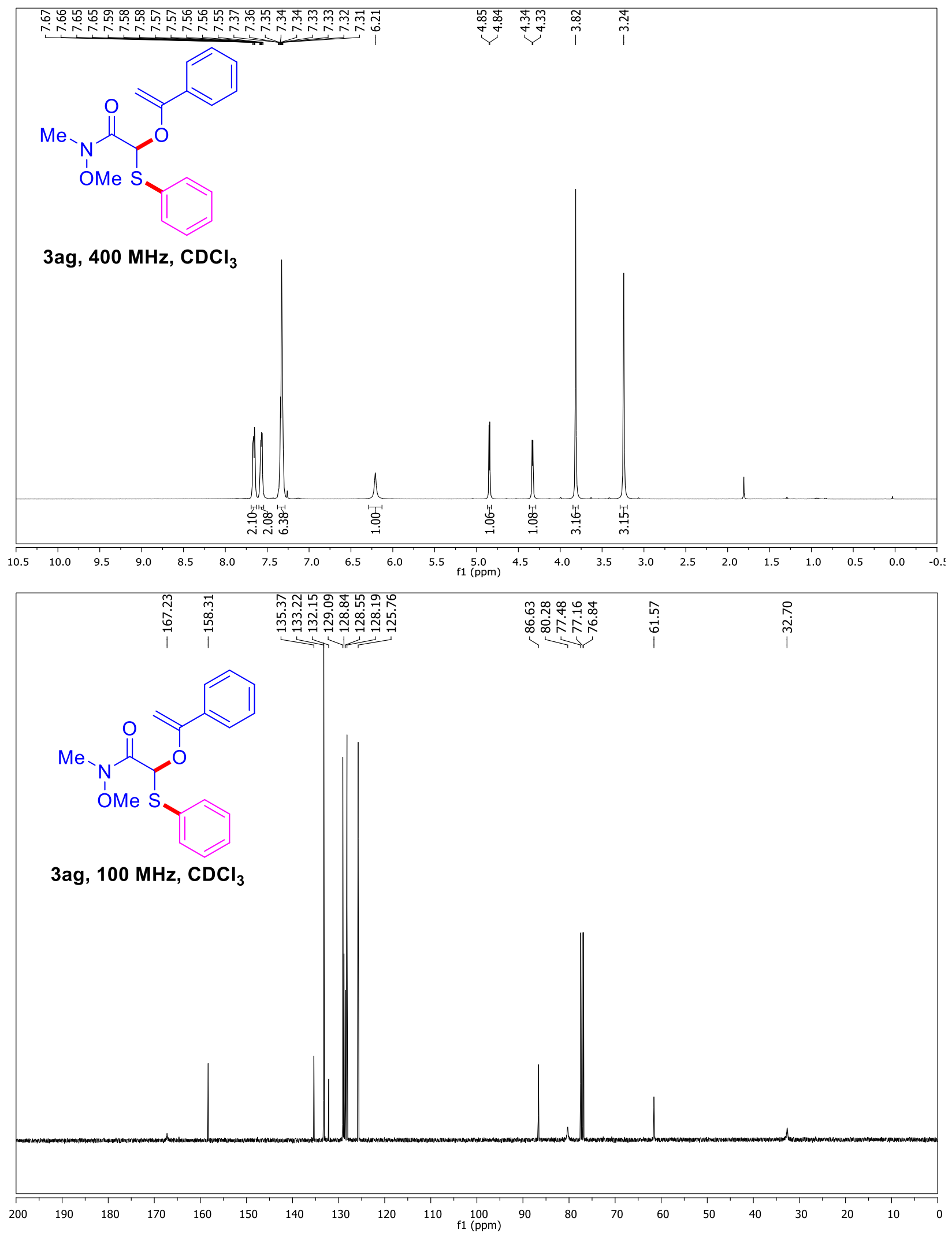
Ethyl-2-(cyclohex-1-en-1-yloxy)-2-(phenylthio)acetate (3ah)

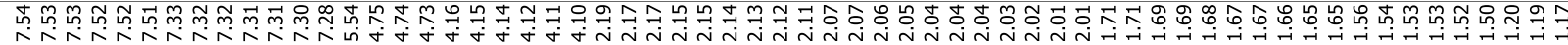<smiles>CCOC(=O)C(OC1=CCCCC1)c1ccccc1</smiles>

3ah, $400 \mathrm{MHz}, \mathrm{CDCl}_{3}$
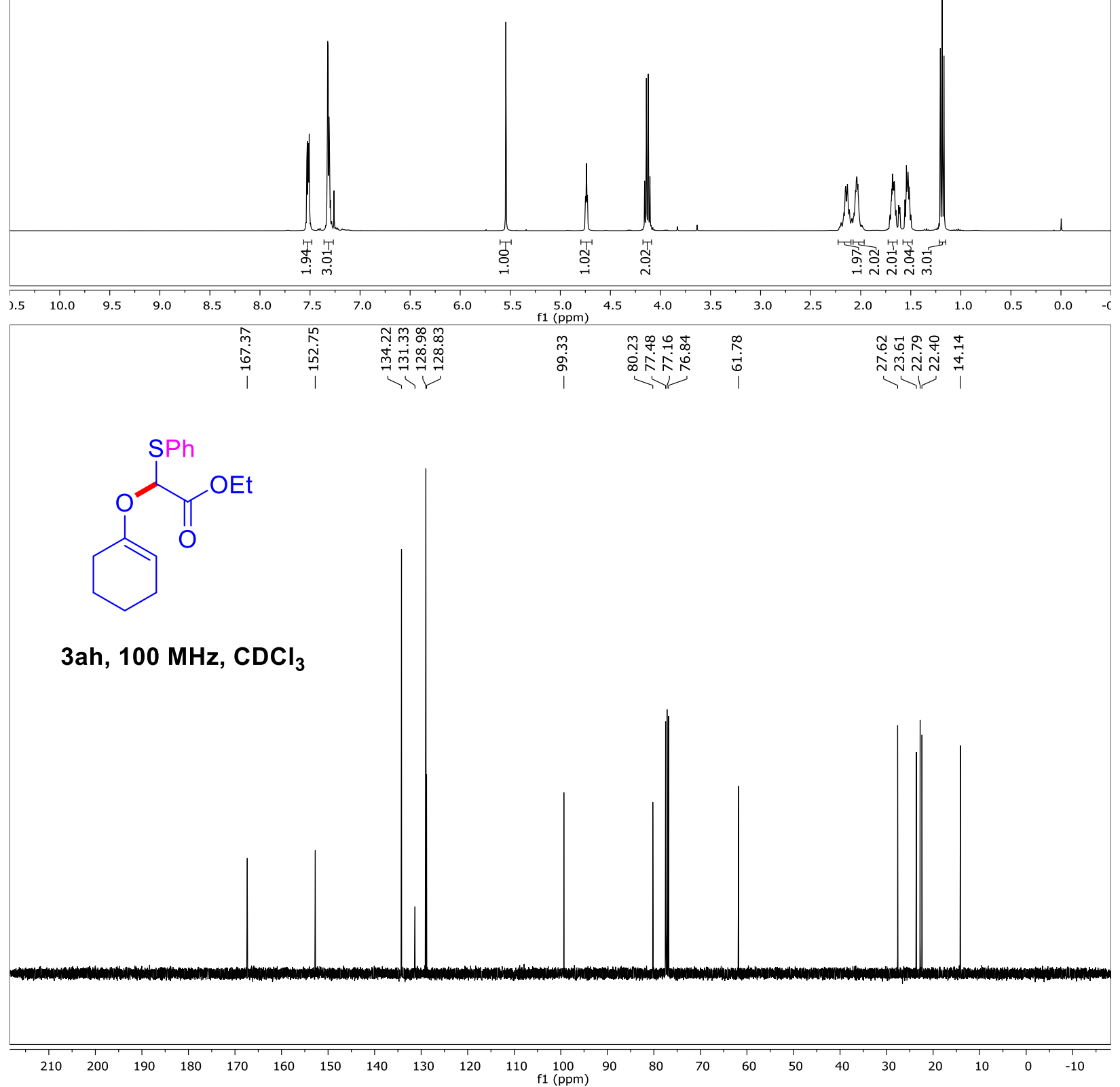
Ethyl 2-(cyclohept-1-en-1-yloxy)-2-(phenylthio)acetate (3ai)
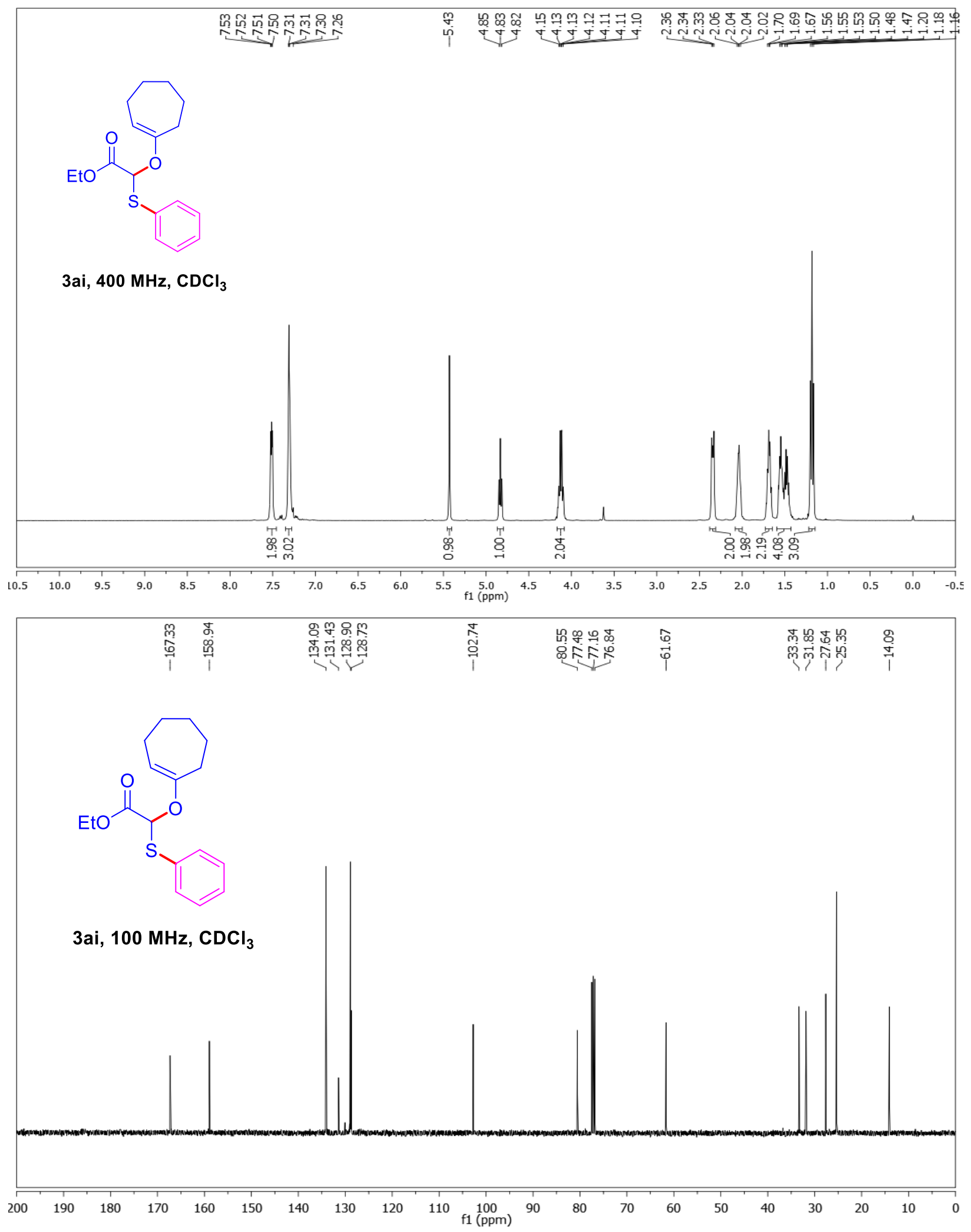
Ethyl-2-((1H-inden-3-yl)oxy)-2-(phenylthio)acetate (3aj)
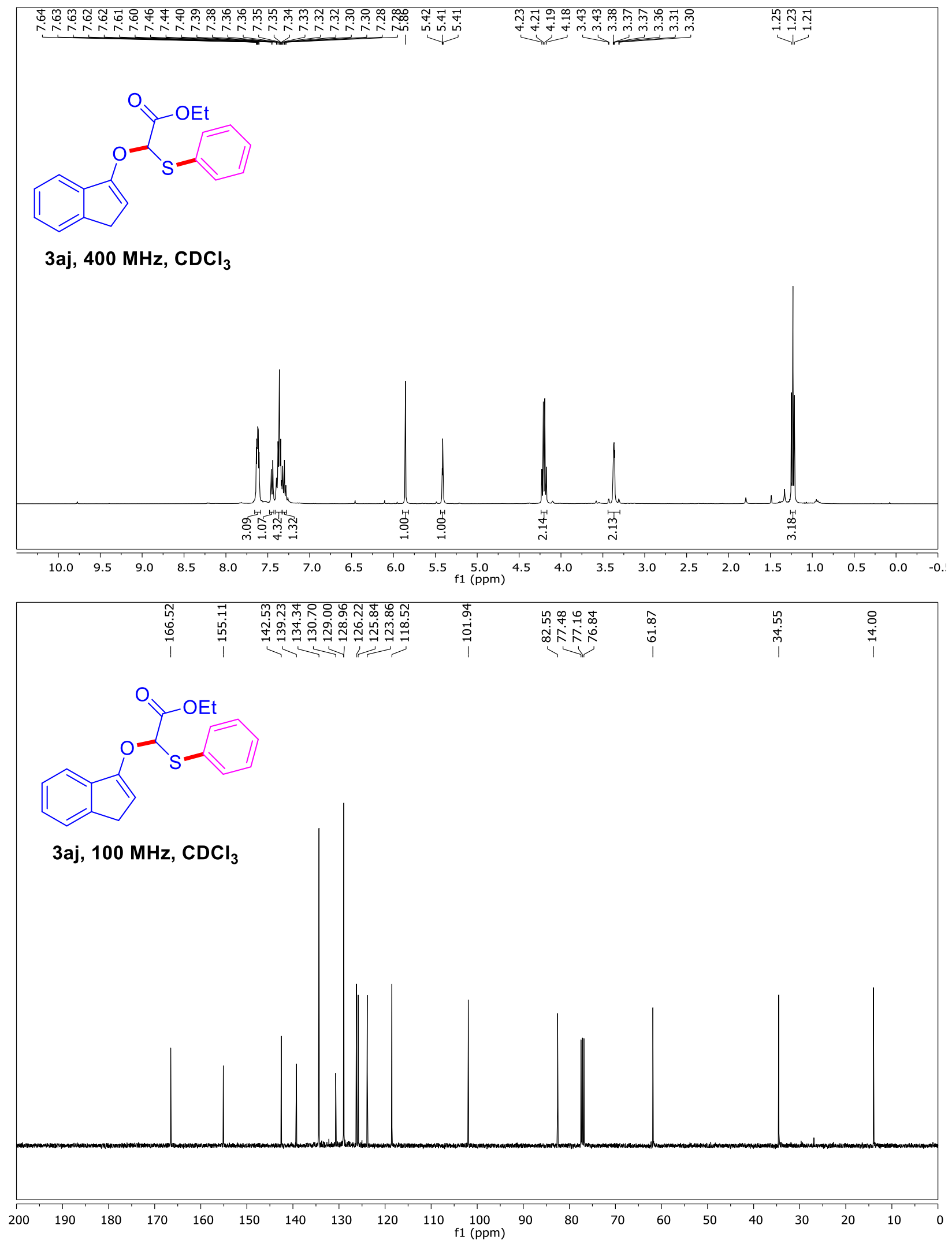

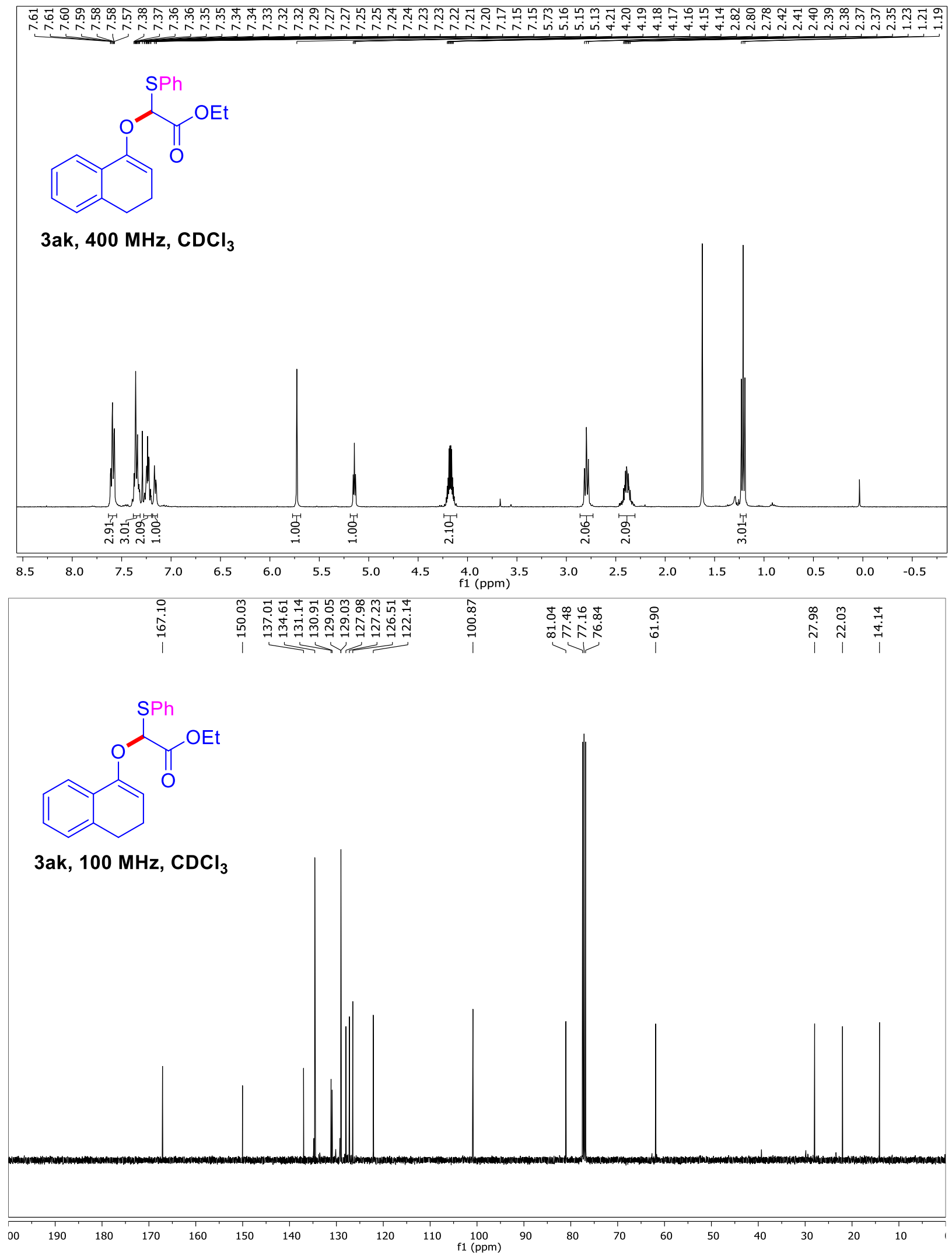
Ethyl-2-(phenylthio)-2-(prop-1-en-2-yloxy)acetate (3al)

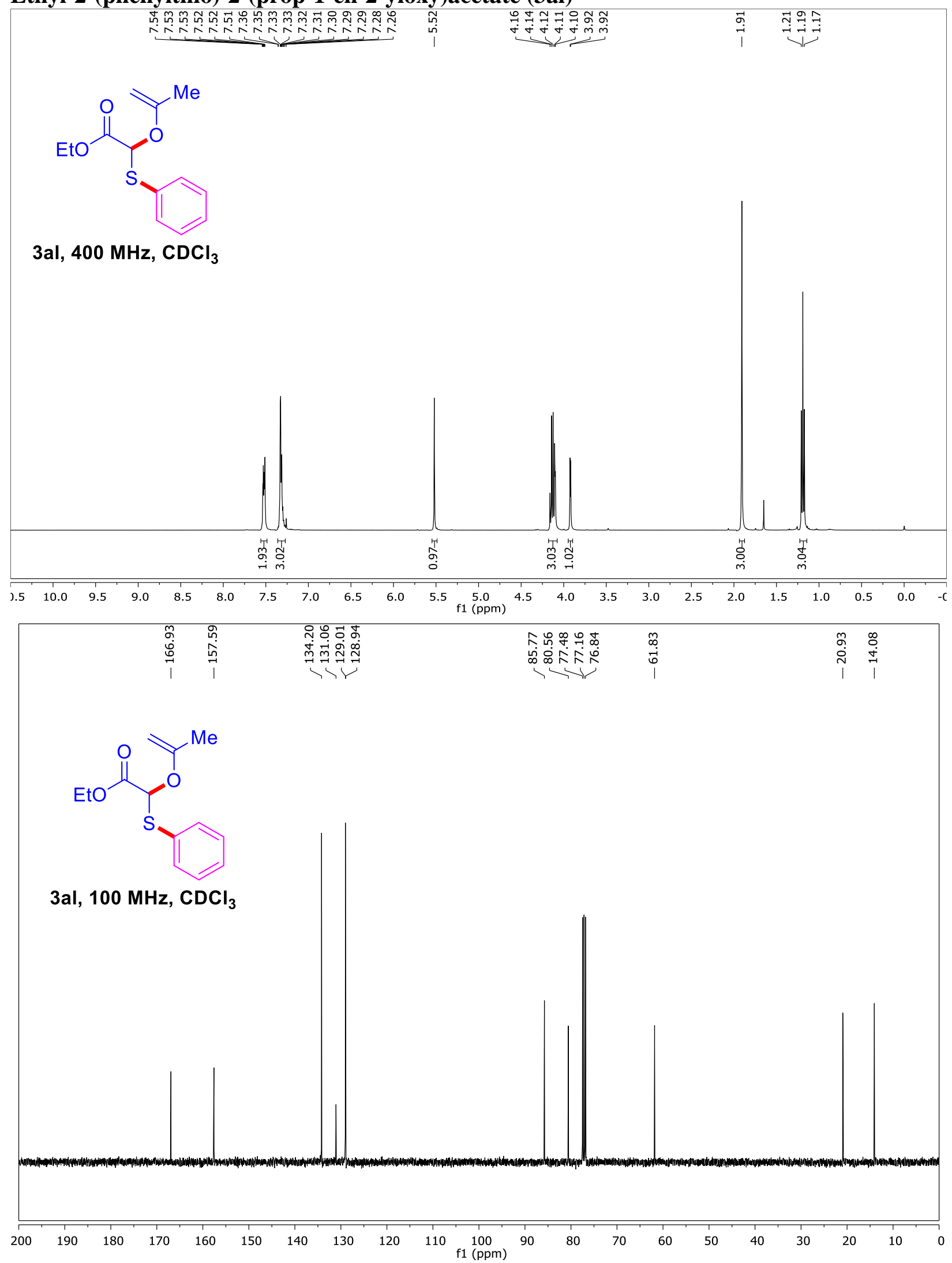


Ethyl-2-((3,4-dimethylphenyl)thio)-2-((1-phenylvinyl)oxy)acetate (3am)
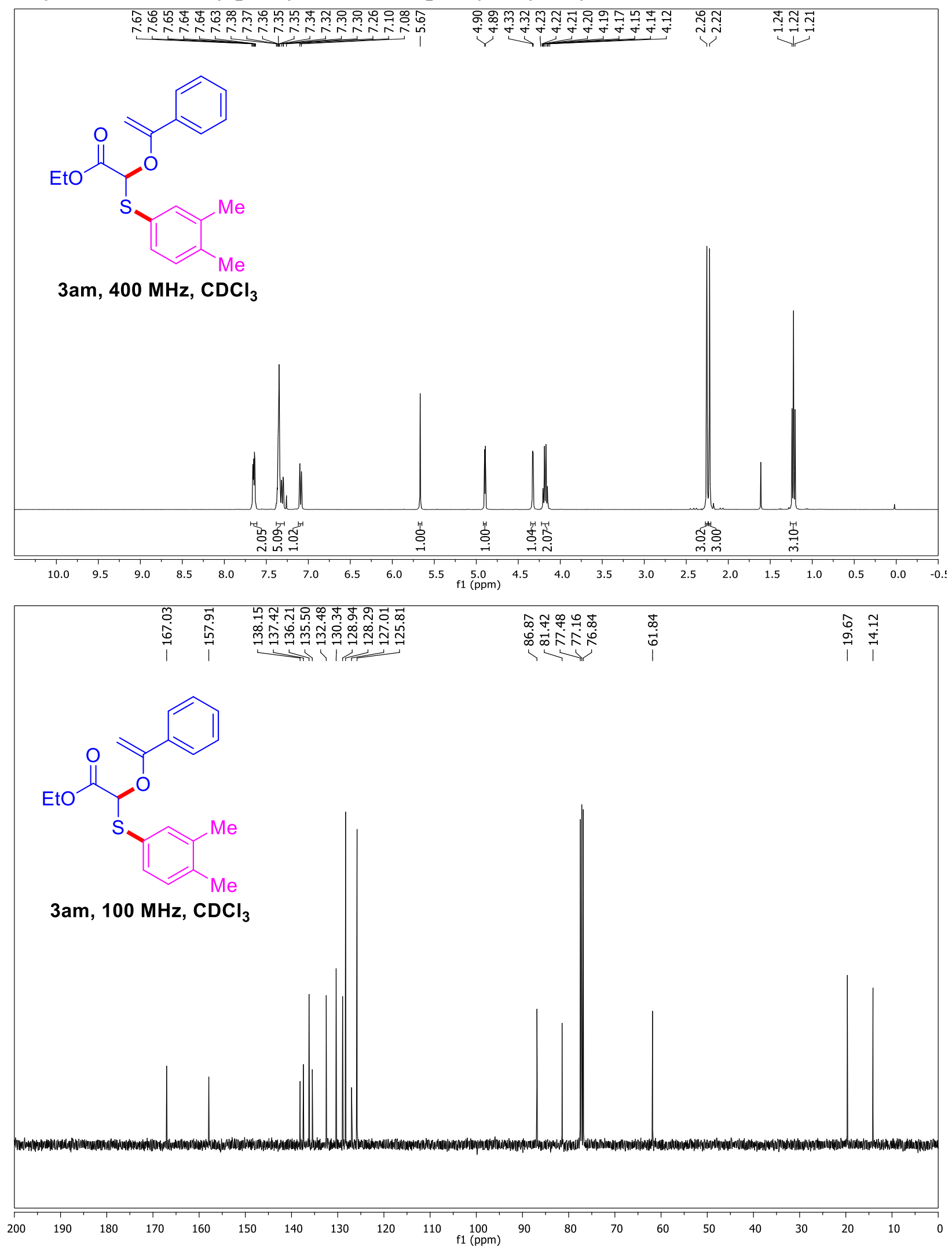
Ethyl-2-(benzo[d][1,3]dioxol-5-ylthio)-2-((1-phenylvinyl)oxy)acetate (3an)
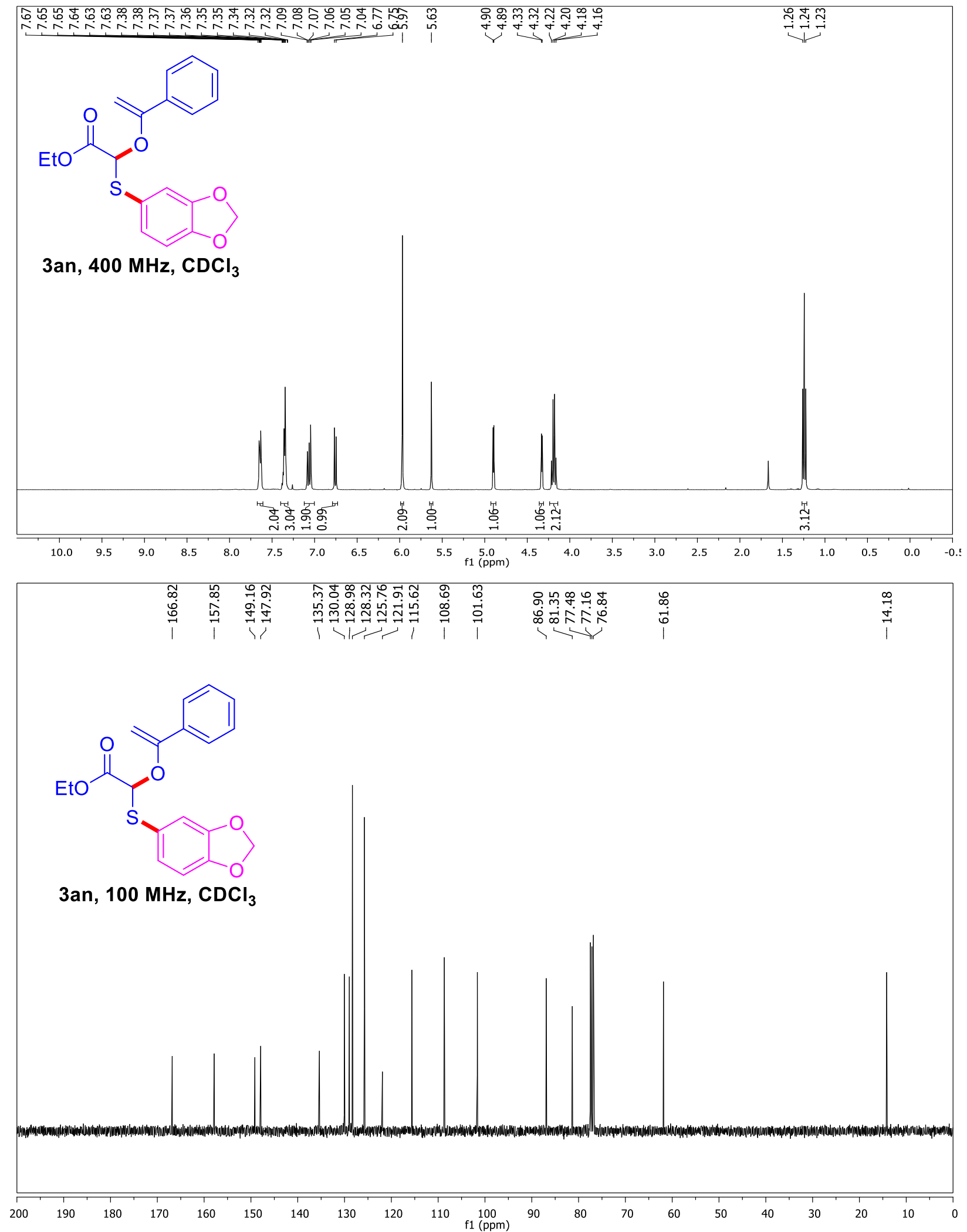
Ethyl-2-((3,4-difluorophenyl)thio)-2-((1-phenylvinyl)oxy)acetate (3ao)
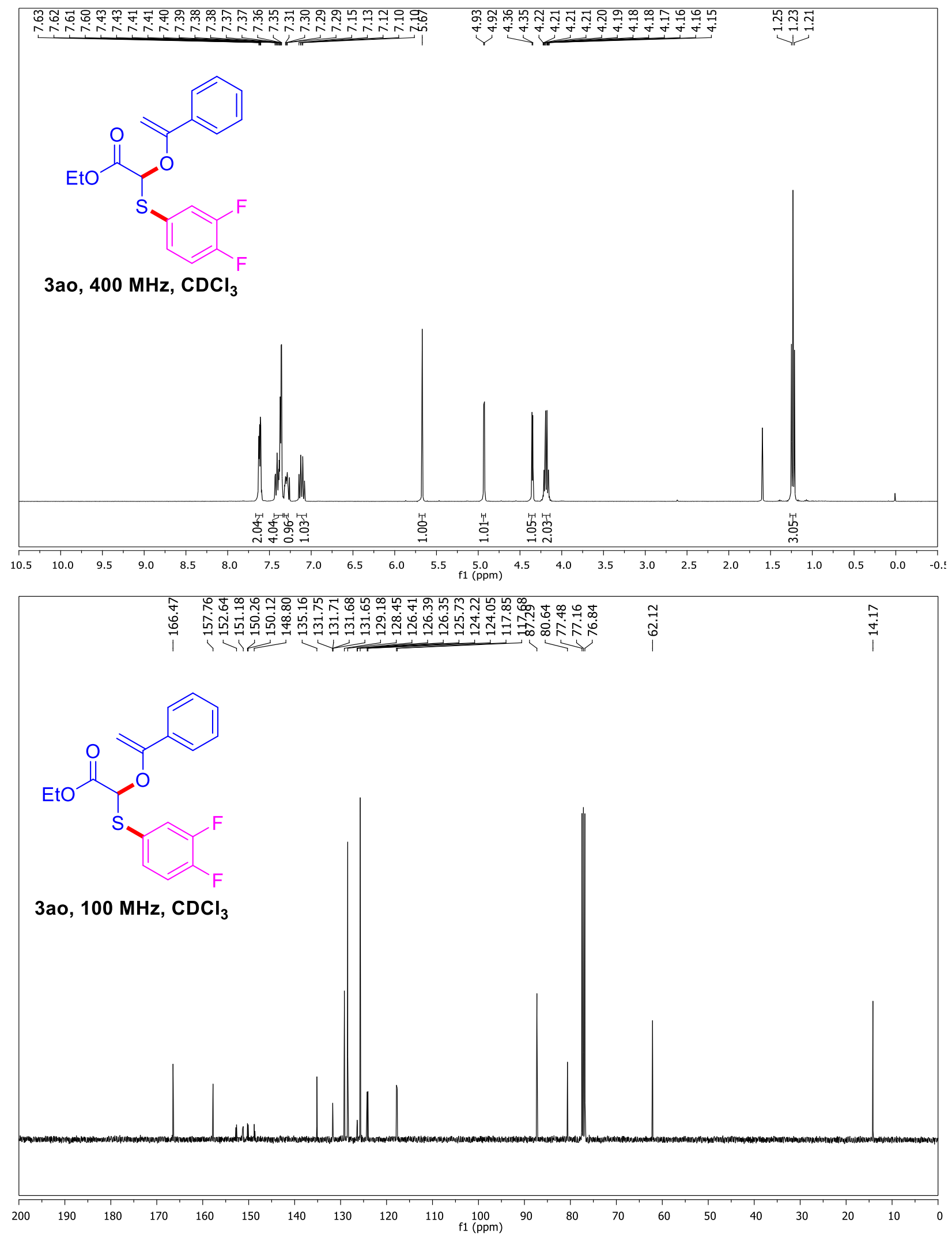
Ethyl-2-((2,3-dihydro-1 $H$-inden-5-yl)thio)-2-((1-phenylvinyl)oxy)acetate (3ap)
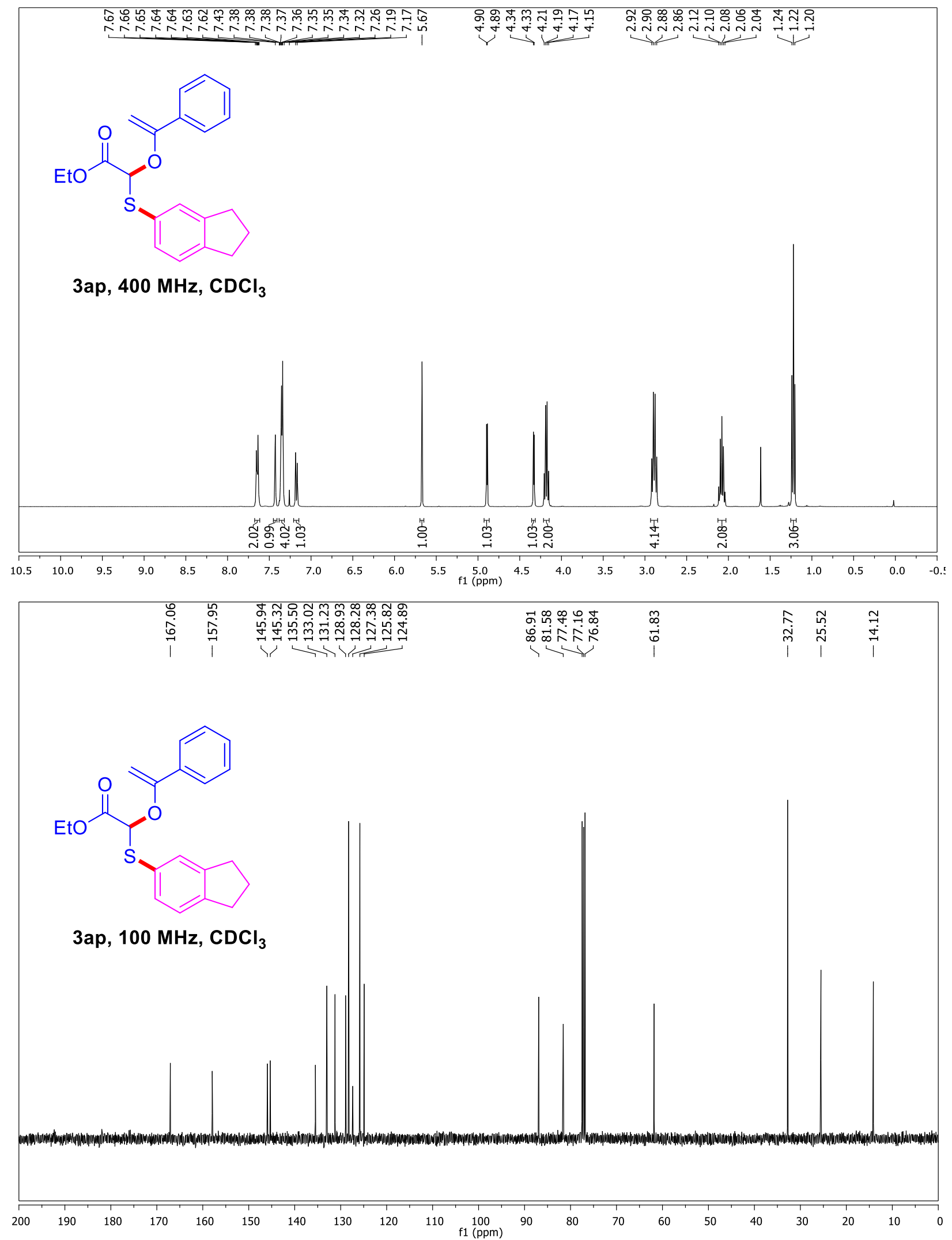
Ethyl-2-((2,5-dimethylphenyl)thio)-2-((1-phenylvinyl)oxy)acetate (3aq)
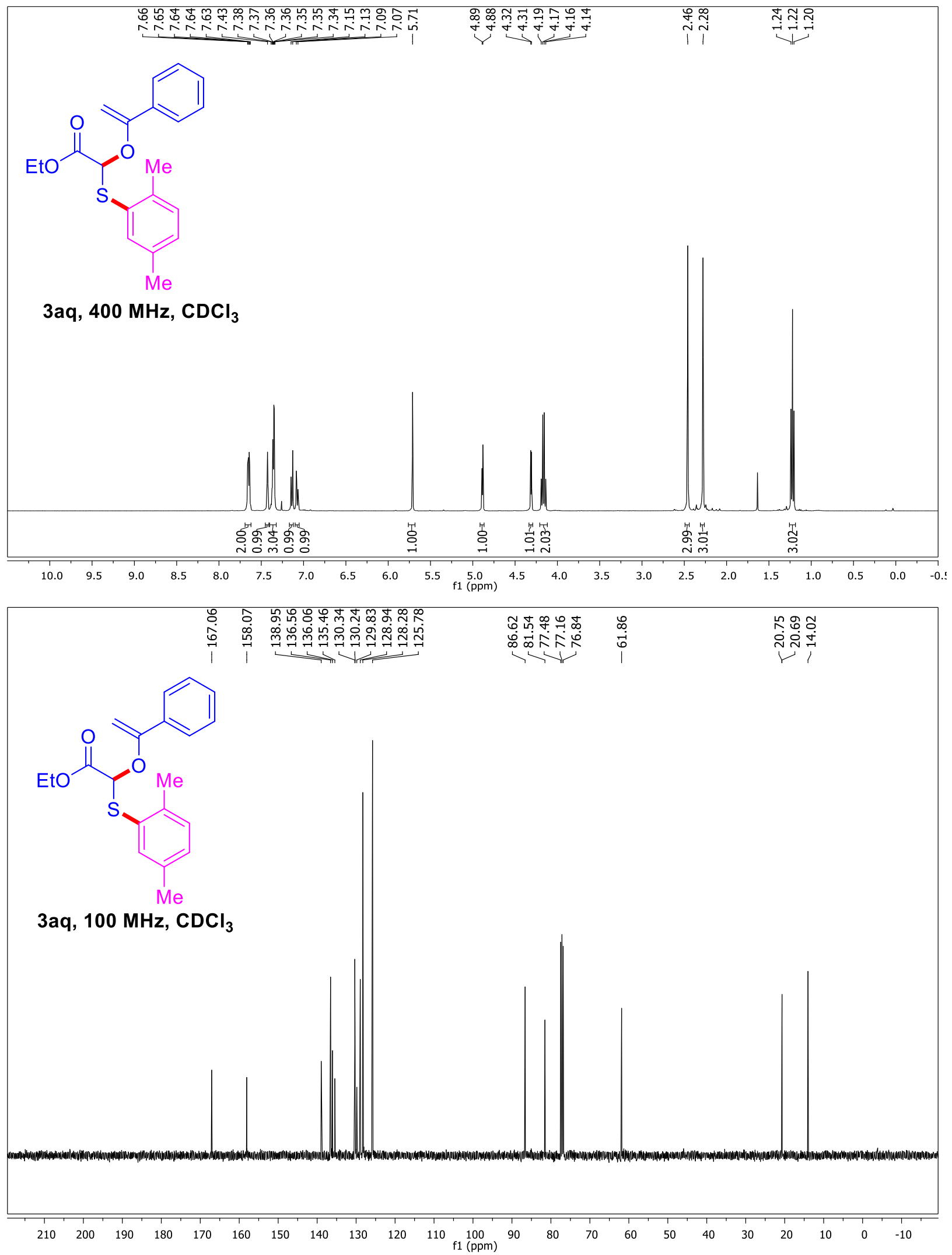
Ethyl-2-(naphthalen-2-ylthio)-2-((1-phenylvinyl)oxy)acetate (3ar)
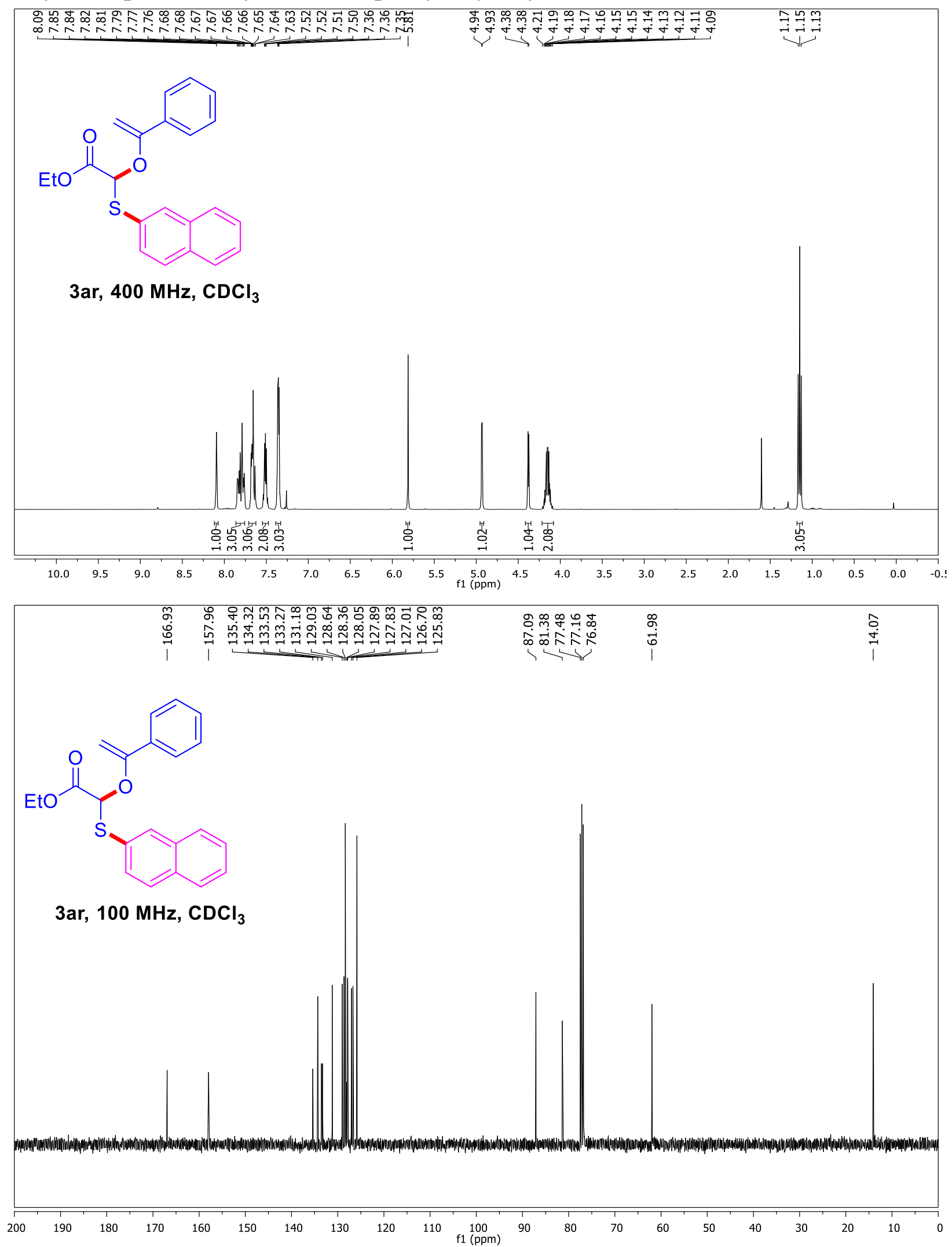
Ethyl-2-(naphthalen-2-ylthio)-2-((1-phenylvinyl)oxy)acetate (3ar) and Ethyl 2-(naphthalen-1-ylthio)-2-((1-phenylvinyl)oxy)acetate (3as)
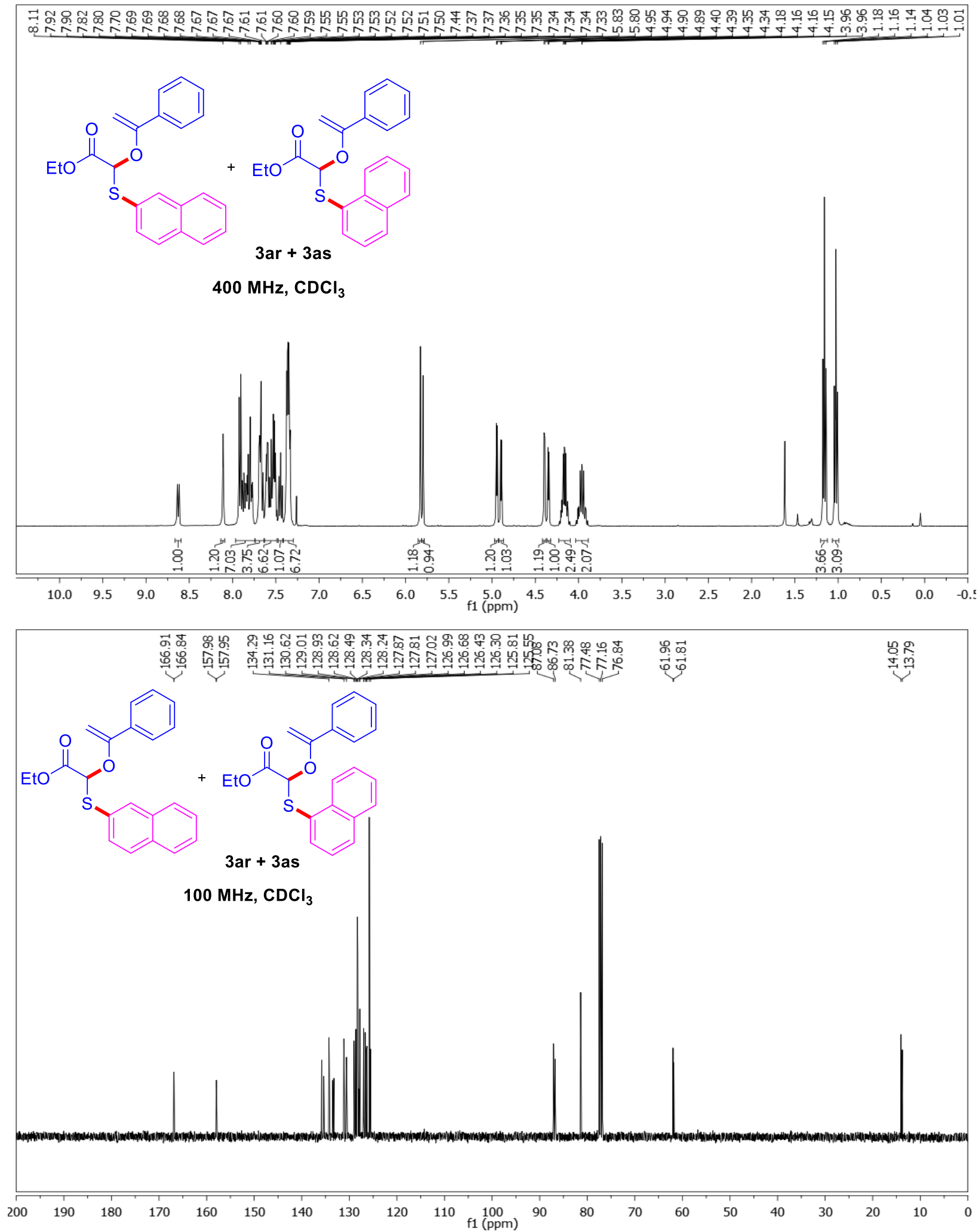
Ethyl-2-((3-methoxyphenyl)thio)-2-((1-phenylvinyl)oxy)acetate (3at)
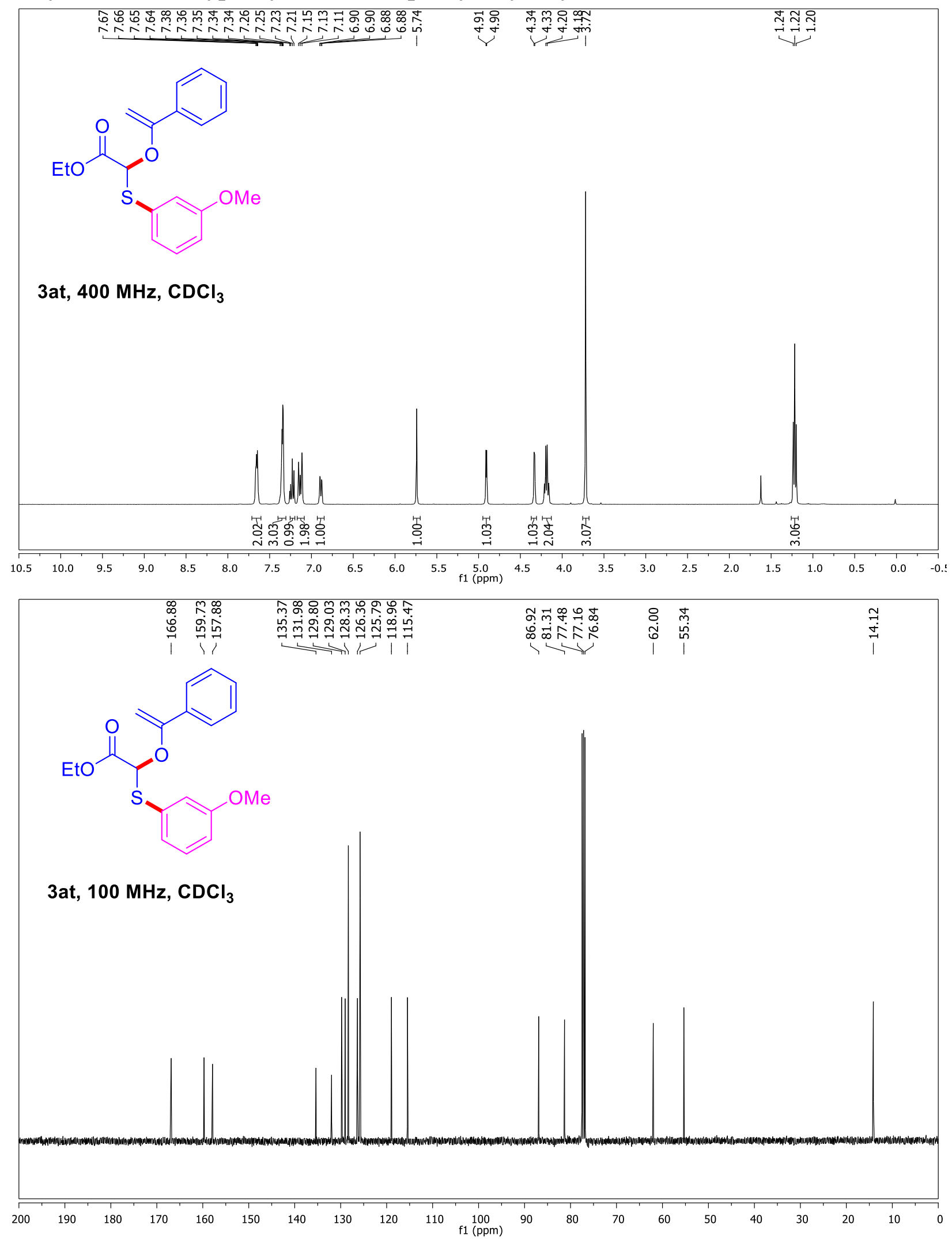
Ethyl-2-((1-phenylvinyl)oxy)-2-(p-tolylthio)acetate (3au) and

Ethyl-2-((1-phenylvinyl)oxy)-2-( $m$-tolylthio)acetate (3au')
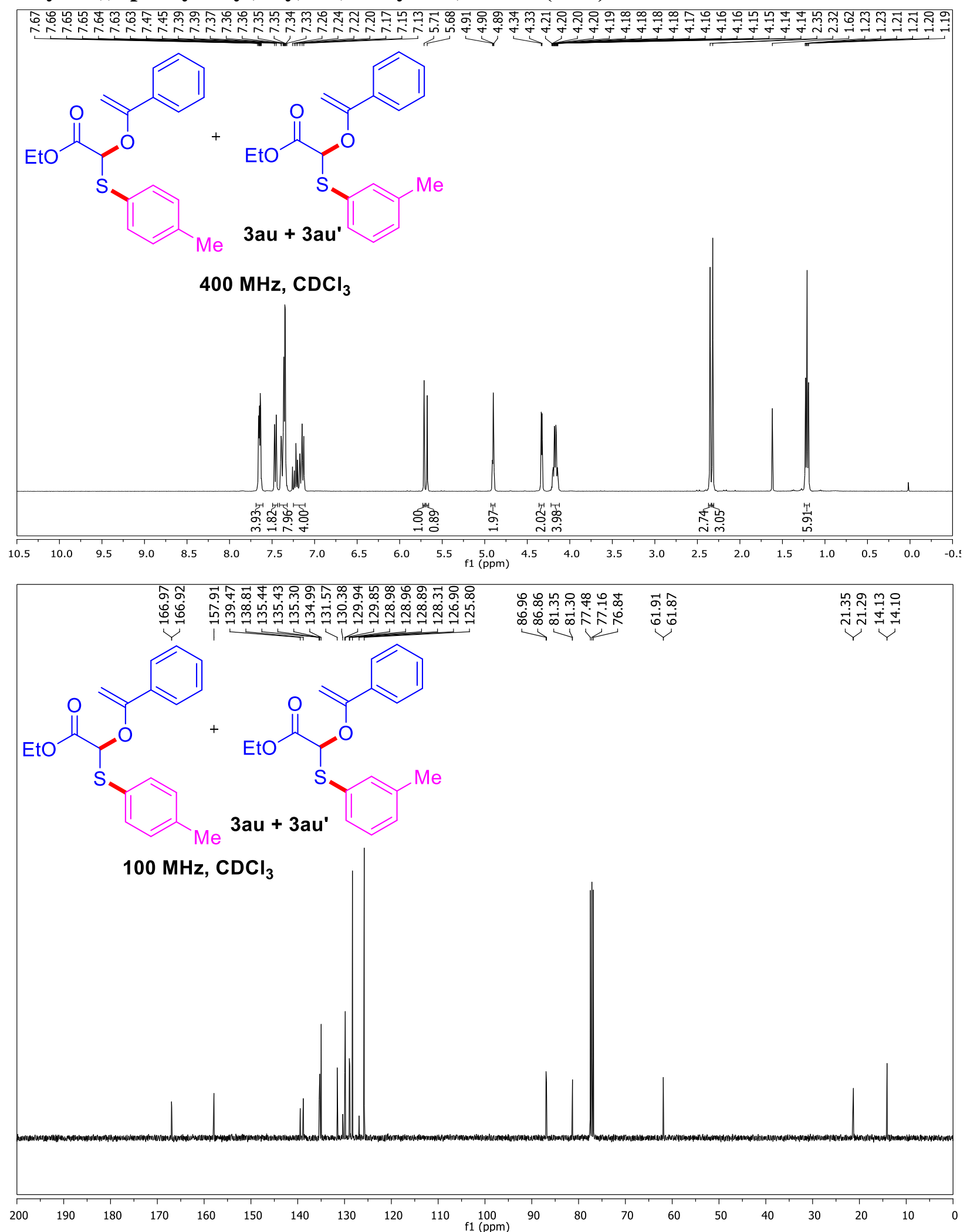
Ethyl-2-((4-chlorophenyl)thio)-2-((1-phenylvinyl)oxy)acetate and Ethyl-2-((3-chlorophenyl)thio)-2-((1-phenylvinyl)oxy)acetate (3av and 3av')
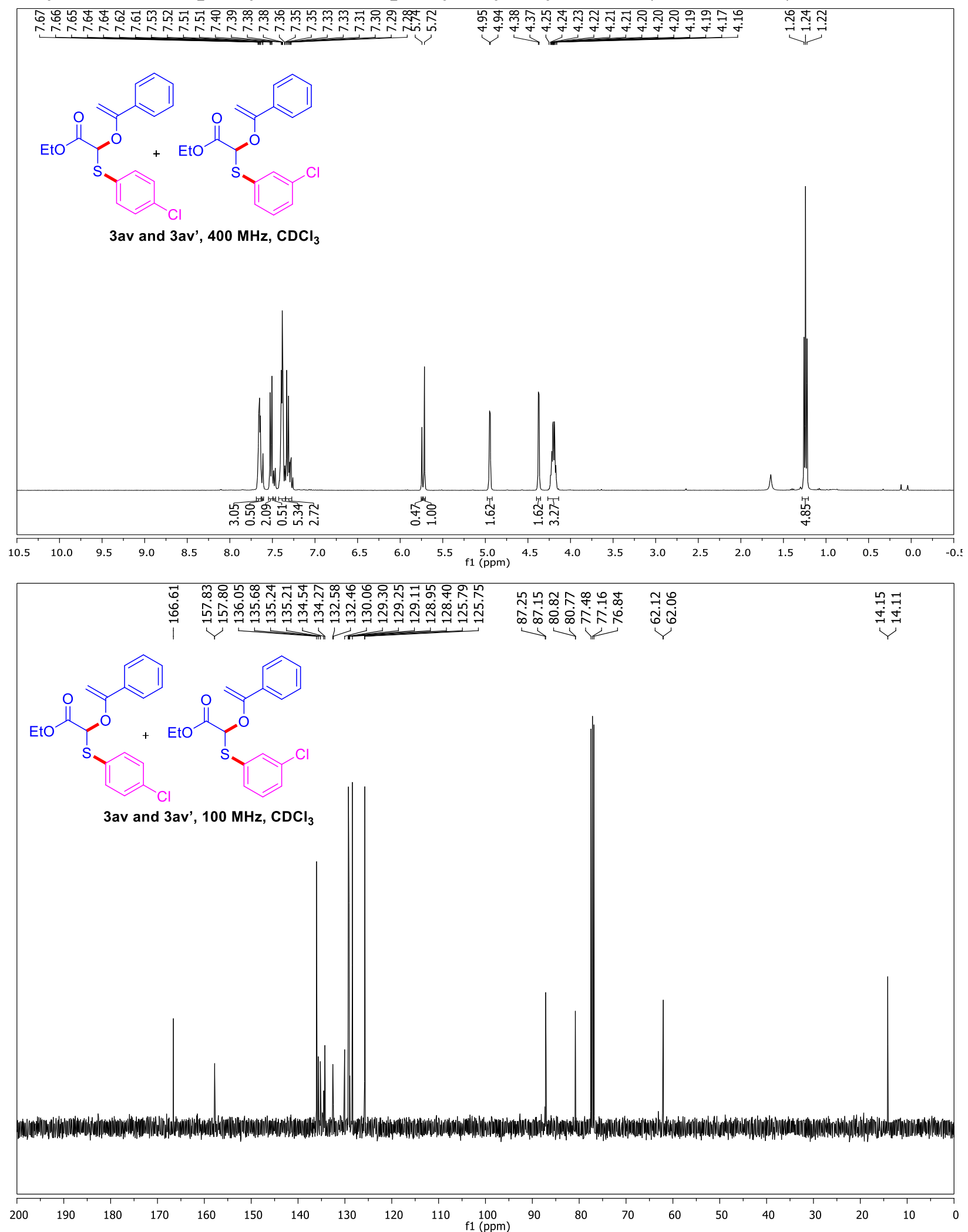
Ethyl-2-((4-fluorophenyl)thio)-2-((1-phenylvinyl)oxy)acetate and Ethyl -2-((3-fluorophenyl)thio)-2-((1-phenylvinyl)oxy)acetate (3aw and 3aw')
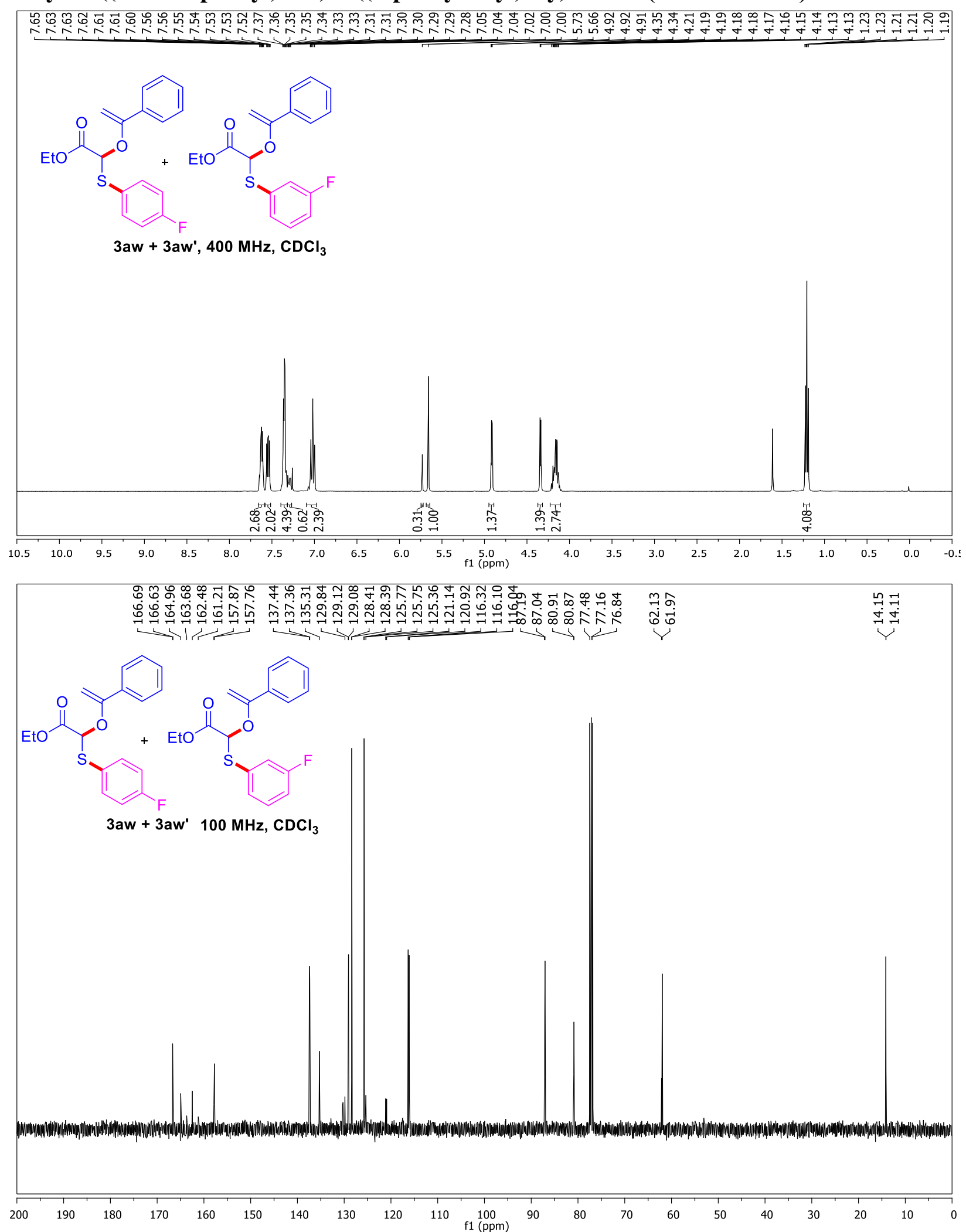
1-Phenyl-2-(phenylthio)-2-((1-phenylvinyl)oxy)ethan-1-one (10a)

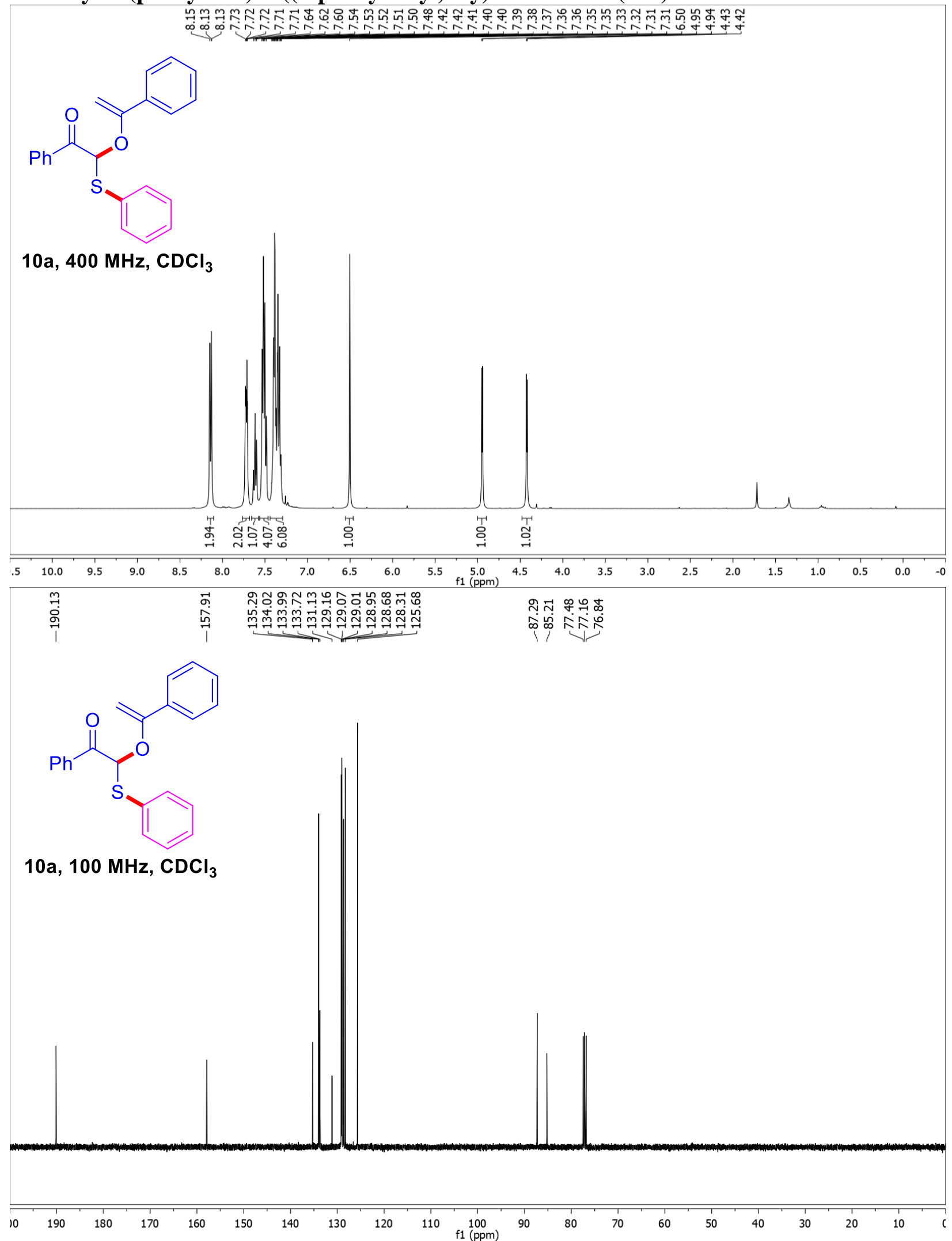


$N$-Methyl-2-(phenylthio)-2-((1-phenylvinyl)oxy)acetamide (11)
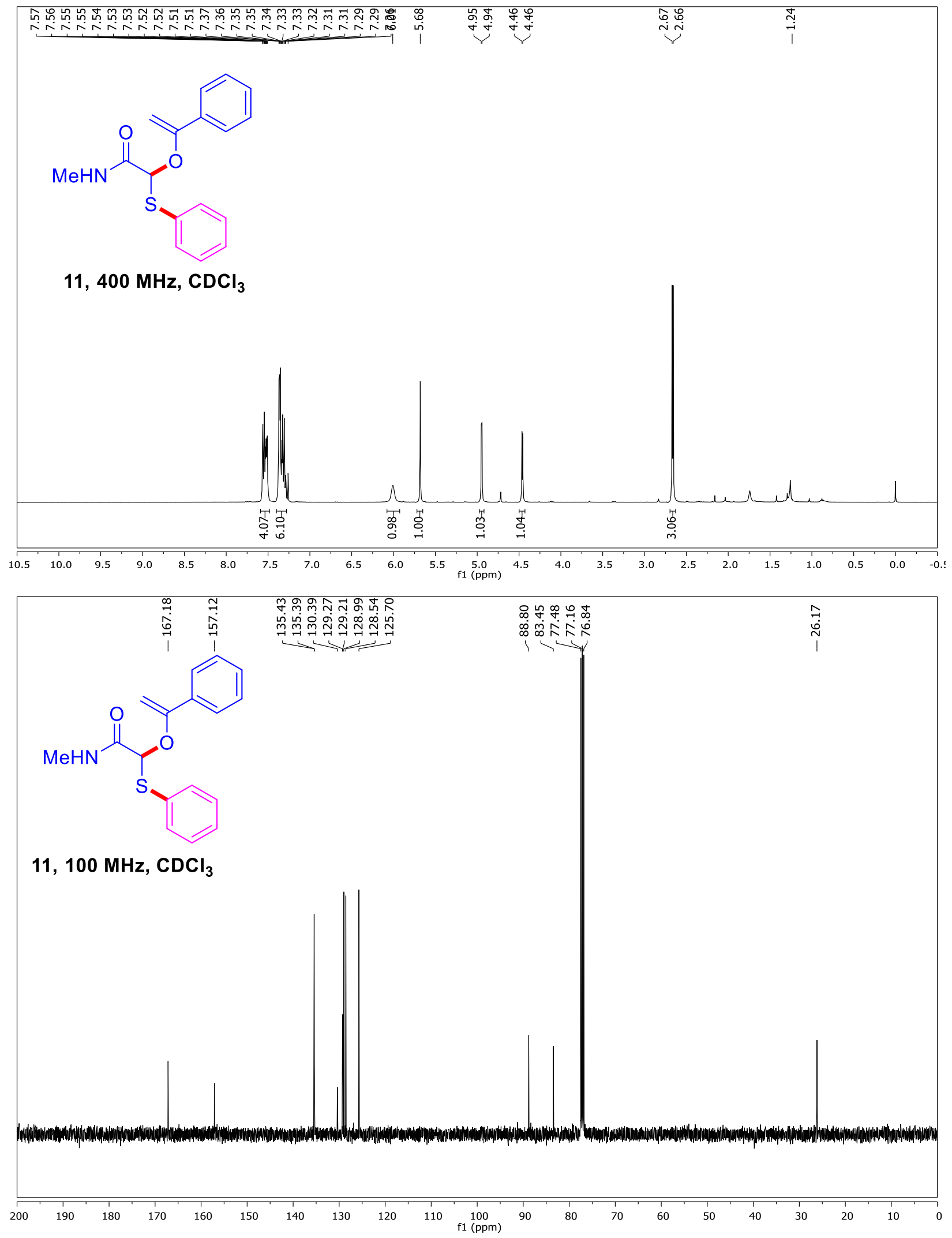
2-((1-(4-Bromophenyl)vinyl)oxy)- $N$-methyl-2-(phenylthio)acetamide (12)

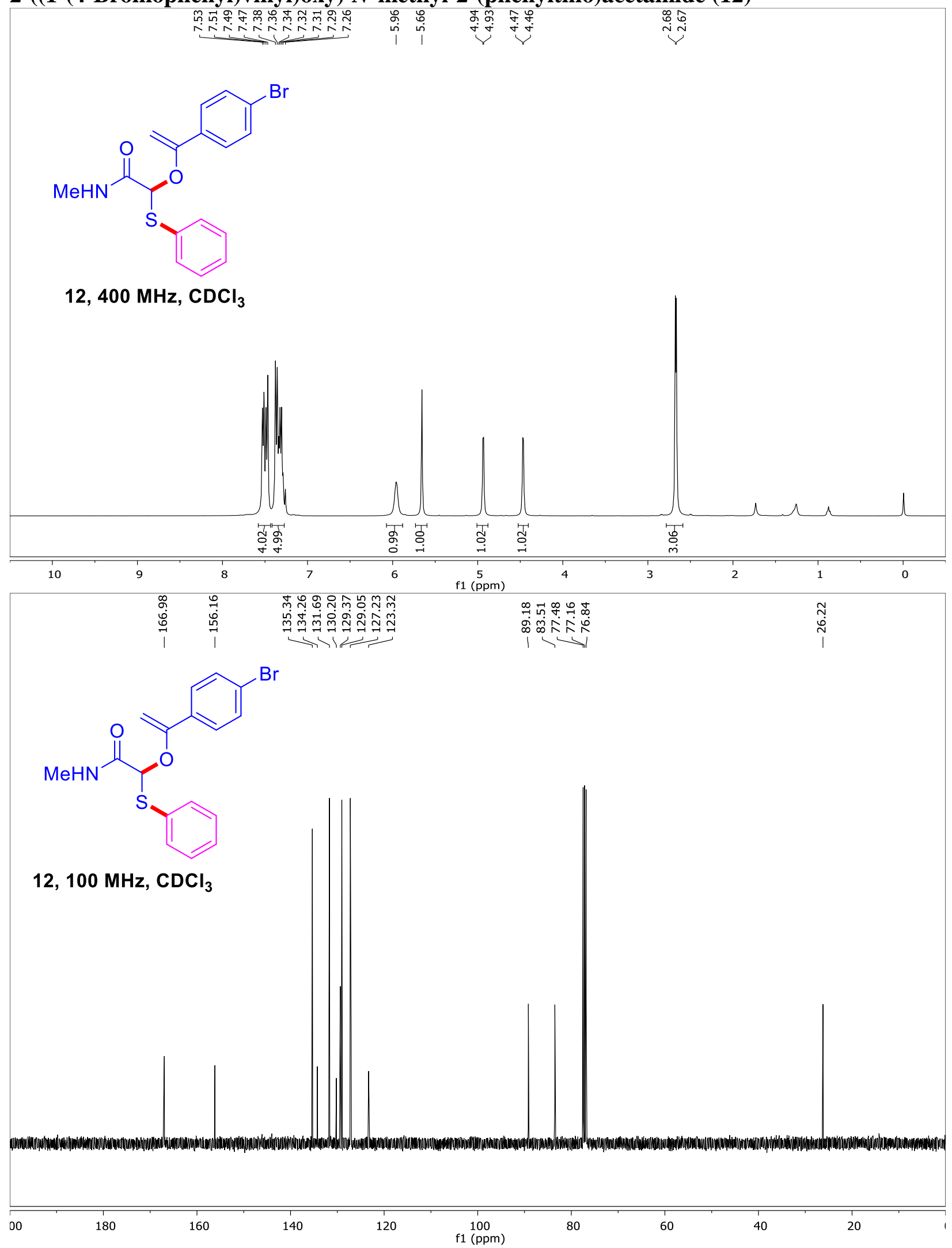


2-Methyl-2-phenyl-5-(phenylthio)-1,3-dioxolan-4-one (13)
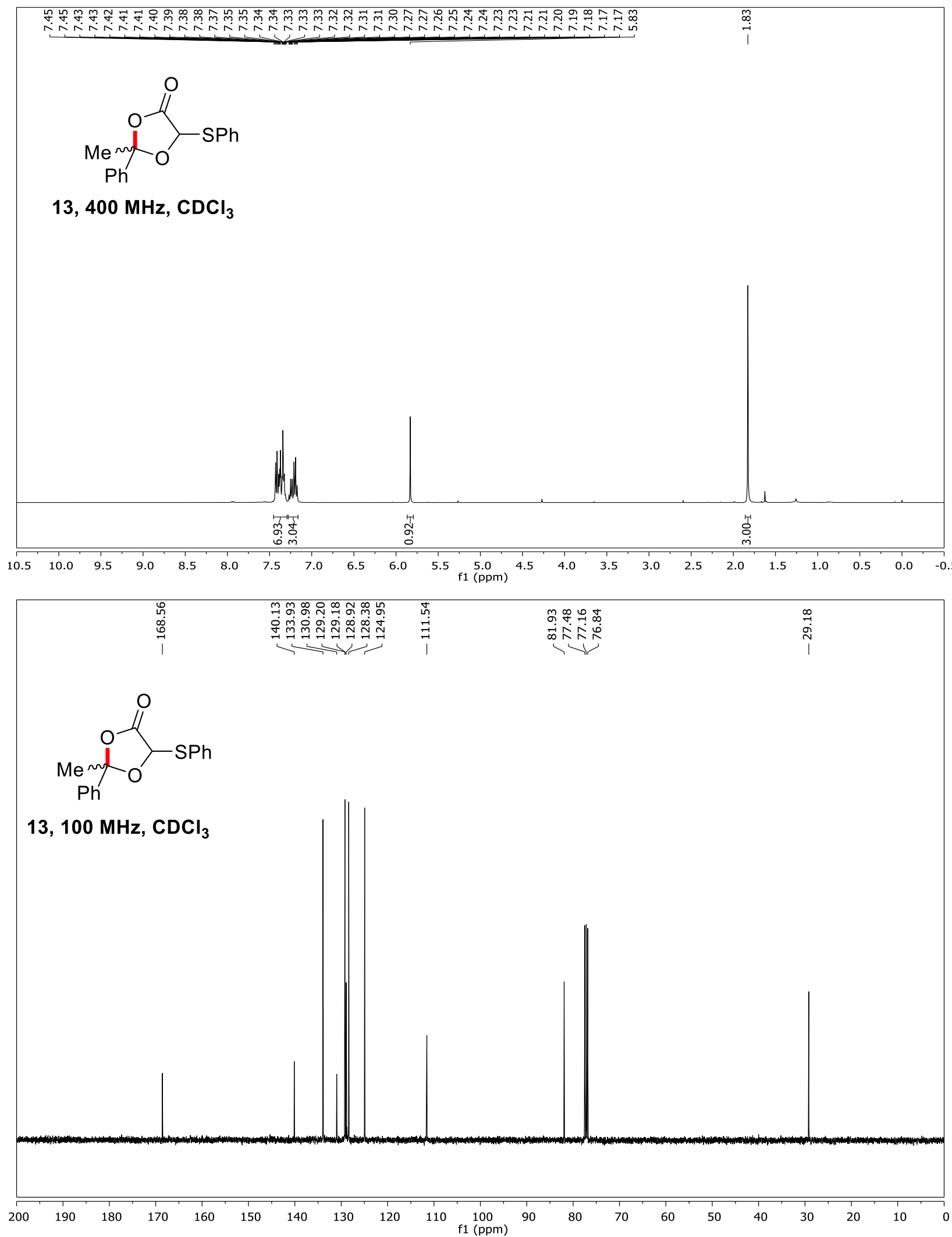
2-Methyl-2-(naphthalen-1-yl)-5-(phenylthio)-1,3-dioxolan-4-one (14)<smiles></smiles>

$14,400 \mathrm{MHz}, \mathrm{CDCl}_{3}$
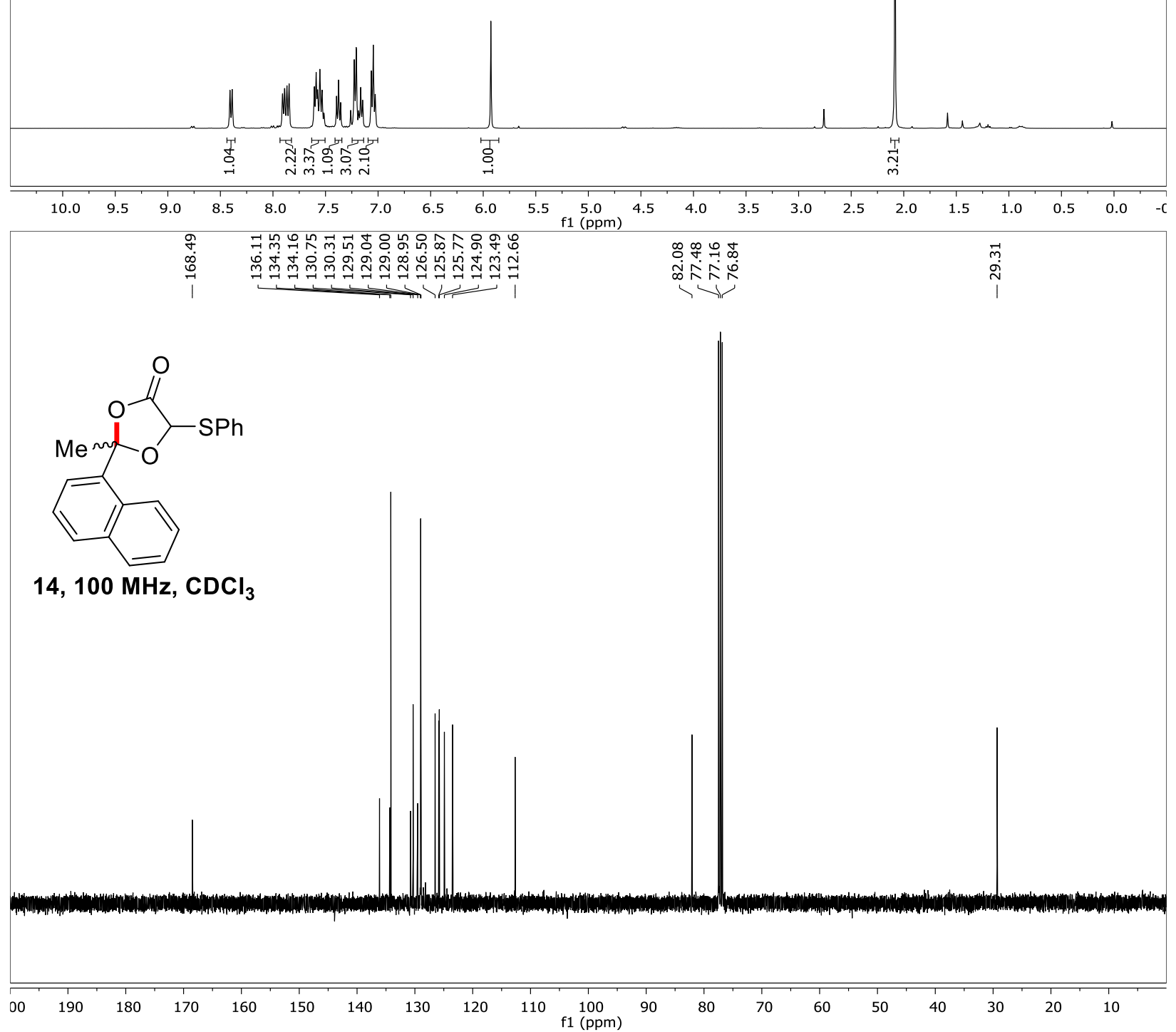